\title{
Modification of white adipose tissue biology and metabolic profiles in humans by nutritional bio-actives
}

Citation for published version (APA):

Warnke, I. (2018). Modification of white adipose tissue biology and metabolic profiles in humans by nutritional bio-actives. [Doctoral Thesis, Maastricht University]. Maastricht University. https://doi.org/10.26481/dis.20180316iw

Document status and date:

Published: 01/01/2018

DOI:

10.26481/dis.20180316iw

Document Version:

Publisher's PDF, also known as Version of record

\section{Please check the document version of this publication:}

- A submitted manuscript is the version of the article upon submission and before peer-review. There can be important differences between the submitted version and the official published version of record.

People interested in the research are advised to contact the author for the final version of the publication, or visit the DOI to the publisher's website.

- The final author version and the galley proof are versions of the publication after peer review.

- The final published version features the final layout of the paper including the volume, issue and page numbers.

Link to publication

\footnotetext{
General rights rights.

- You may freely distribute the URL identifying the publication in the public portal. please follow below link for the End User Agreement:

www.umlib.nl/taverne-license

Take down policy

If you believe that this document breaches copyright please contact us at:

repository@maastrichtuniversity.nl

providing details and we will investigate your claim.
}

Copyright and moral rights for the publications made accessible in the public portal are retained by the authors and/or other copyright owners and it is a condition of accessing publications that users recognise and abide by the legal requirements associated with these

- Users may download and print one copy of any publication from the public portal for the purpose of private study or research.

- You may not further distribute the material or use it for any profit-making activity or commercial gain

If the publication is distributed under the terms of Article $25 \mathrm{fa}$ of the Dutch Copyright Act, indicated by the "Taverne" license above, 
Modification of white adipose tissue biology

and metabolic profiles in humans

by nutritional bio-actives 
The studies presented in this thesis were performed within NUTRIM School of Nutrition and Translational Research in Metabolism, which participates in the Graduate School VLAG (Food Technology, Agrobiotechnology, Nutrition and Health Sciences), accredited by the Royal Netherlands Academy of Arts and Sciences, and within the Department of Human Nutrition and Health at DSM Nutritional Products, Basel Switzerland.
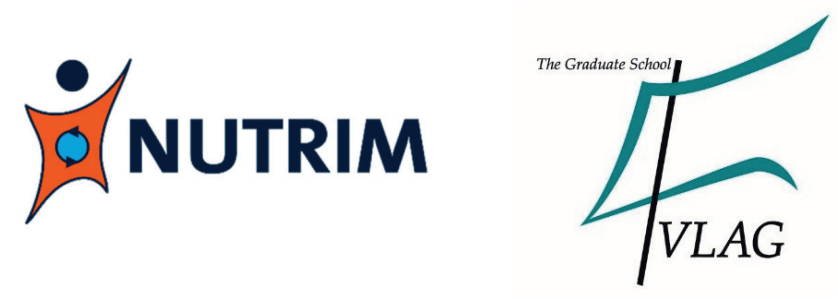

The research described in this thesis was supported by DSM Nutritional Products, and by a grant from the Alpro Foundation.

\section{DSM}

BRIGHT SCIENCE. BRIGHTER LIVING.

\section{alpro Foundation Knowledge in plant-based nutrition}

(C) Ines Warnke, Basel 2017

Cover design: Sofia G and Ines Warnke

Layout: Ines Warnke

Printed by: ProefschriftMaken || www.proefschriftMaken.nl

ISBN: 978-94-6295-861-6 


\title{
Modification of white adipose tissue biology and metabolic profiles in humans by nutritional bio-actives
}

\author{
PhD thesis \\ to obtain the doctor degree \\ at Maastricht University, \\ under the authority of Rector Magnificus, Prof.Dr.L.L.G.Soete \\ according to the decision of the College of Decans, \\ to defend in public \\ on Friday $16^{\text {th }}$ March 2018 at $2.00 \mathrm{pm}$
}

by

Ines Warnke

born in Ludwigslust on May 2, 1979 


\section{Supervisors}

Prof. Wim H.M. Saris

Prof. Ellen E. Blaak

\section{Co-Supervisors}

Dr. Johan W.E. Jocken

Dr. Joseph Schwager

\section{Assessment Committee}

Prof. Dr. Edwin C.M. Mariman (chair)

Prof. Dr. Aalt Bast

Prof. Dr. Michael Müller (University of East Anglia, Norwich, UK)

Prof. Casper G. Schalkwijk

Prof. Hubert Vidal (INSERM and CarMeN Lab at Lyon University, France) 


\section{Contents}

$\begin{array}{lll}\text { CHAPTER } 1 \quad \text { General Introduction } & 7\end{array}$

CHAPTER 2 Dietary constituents reduce lipid accumulation in murine $\mathbf{4 5}$

C3H10 T1/2 adipocytes: A novel fluorescent method to quantify fat droplets

CHAPTER 3 Combinations of bio-active dietary constituents affect human white adipocyte function in-vitro

CHAPTER 4 Effects of combining resveratrol with lycopene on functional features of in-vitro differentiated human white adipocytes

CHAPTER 5 Combined epigallocatechin-3-gallate and resveratrol supplementation for $12 \mathrm{wk}$ increases mitochondrial capacity and fat oxidation, but not insulin sensitivity in obese humans: a randomized controlled trial

CHAPTER 6 The effects of polyphenol supplementation on adipose tissue morphology and gene expression in overweight and obese humans

CHAPTER 7 General Discussion

Summary

Zusammenfassung

Valorization

Acknowledgements / Danksagungen

Publications

Curriculum Vitae 


\section{CHAPTER 1}

\section{General Introduction}

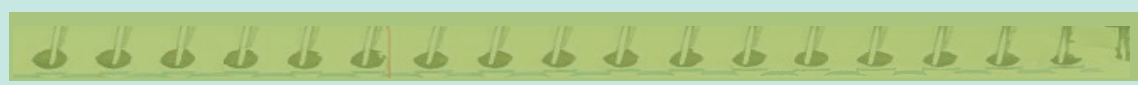

Ines Warnke, Joseph Schwager, Wim Saris, Ellen E. Blaak, Johan W.E. Jocken To be submitted 
Adipose tissue (AT) stores and harbors fat after excessive energy uptake. For a long time, AT has been considered as simple-structured, inert storage organ. But AT is not only a dull structure; exciting biochemical reactions and structural changes happen when the body has been fasting e.g., AT breaks down fat to generate and release energy in the form of fatty acids (FAs). During famine, only people with enough storage fat survive. In the early period of investigations, research focused on the fat storage and breakdown in adipocytes, the most prominent cell type in AT. In the last 20 years, scientific advances revealed that AT is a rather complex organ with major biological, metabolic, and endocrine functions [1].

White adipose tissue (WAT) has a remarkable capacity to expand due to fat storage in times of caloric excess [2], leading to the most obvious phenomenon of obesity, i.e. expanded WAT, reshaping the body. Research focusing on AT biology has enormously progressed due to the increasing prevalence of obesity $[3,4]$ probably attributable to an unlimited and easy access to palatable, energy-dense, processed foods [5], decreased physical activity and sedentary lifestyle [6]. According to the World Health Organization (WHO), obesity is defined as medical condition with an abnormal accumulation of body fat, which can lead to an impairment of health and has worldwide more than doubled since 1980 [7]. In 2014, more than 1.9 billion (39\%) adults (>18 years) were classified as overweight (body mass index (BMI) $>25 \mathrm{~kg} / \mathrm{m} 2$ ), of these over 600 million $(13 \%)$ people were obese (BMI $>30 \mathrm{~kg} / \mathrm{m} 2)$, and 41 million children under the age of 5 were overweight or obese [7]. In Europe, obesity affects more than 100 million people and occurs more frequent in socio-economically underprivileged populations [7].

Since 1998, when the WHO highlighted the "appearance" of obesity in the field of modern medicine, (metabolic) diseases are increasingly associated with this medical condition. Among them are type-2-diabetes mellitus (T2DM) [8], cardiovascular diseases (CVD) [9], musculoskeletal disorders [7], respiratory complications [10], non-alcoholic fatty liver disease (NAFLD) [11], several forms of cancer [12] and dementia [13], which cause increased morbidity, disability, premature death, and an increasing burden for health care systems. This advancing prevalence has driven the search for strategies to prevent and treat metabolic derangements by reducing WAT mass e.g., by lifestyle (diet and exercise) interventions [14] or pharmacological effectors of (WAT) metabolism [15] and also intensified the search for dietary factors that might improve obesity-related dysfunctions [16, 17]. Nutritional constituents such as polyphenols, carotenoids, or polyunsaturated fatty acids (PUFAs) are dietary substances that are precursors of, or directly act as, signaling molecules and have been described to modulate adipocyte functions (for references see section "Nutritional bio-actives and AT (dys)-functions"). 
This introduction will firstly summarize the complex functions of AT and the influence of dysfunctional AT on other insulin sensitive organs and overall metabolic health. Secondly, the potential effects of dietary substances with partly distinct biological activity (incl. polyphenols, carotenoids and PUFAs), so called nutritional bio-actives, on adipocyte and AT (dys)-functions and metabolic health will be discussed. Finally, an overview of the chapters described in this thesis will be presented.

\section{Adipose Tissue (AT) Function}

The adipose organ is one of the largest organs in the human body and a healthy lean young adult ( $\sim 75 \mathrm{~kg}$ ) stores around $10-18 \mathrm{~kg}$ of fat $(\sim 13-25 \%$ of total body weight), representing 90000$162000 \mathrm{kcal}$, enabling him/her to survive 60-70 days of starvation [1].

\section{Types, depots, and key features}

The human adipose organ consists of white (WAT), "beige" (bAT) and brown adipose tissue (BAT), which is distributed depending on sex, age and response to external and internal factors, in several distinct depots [18]. WAT is largely located subcutaneously (scAT) and viscerally (VAT) / internally including omental, mesenteric, and pericardial depots and accommodates within itself bAT [19], whereas small BAT depots can be found in the upper posterior body of adults [20-22]. Adipose tissues and depots differ (Table 1.1) in their structure, gene expression profile [23], metabolic response (lipolytic stimuli) and intrinsic functionality, which are also partly affected by age, gender, and BMI [24, 25].

The major function of WAT is to store and release FAs depending on energy intake and expenditure. Dietary FAs are stored in the form of triacylglycerols (TAGs) in intracellular lipid droplets (LD) and are released by lipolysis (Figure 1.1). This lipid turnover of WAT is regulated by a tight alignment between adipogenic differentiation, lipogenesis and lipolysis as will be delineated in more detail further below (section "Adipocyte differentiation and lipid metabolism"). Furthermore, WAT contributes, beside the skeletal muscles (SM) and liver, to whole-body glucose/energy homeostasis and systemic insulin sensitivity. In brief, in the postprandial state, available glucose is taken up into SM and AT by insulin stimulation, which increases the translocation of the glucose transporter (GLUT)-4 (SLC2A-4) to the plasma membrane and facilitates glucose diffusion into the cells [26]. Although, AT only contributes a minor but significant part to the insulin-mediated whole-body glucose disposal in healthy humans (10-15\%) [27], gain- and loss-of-function studies in mice demonstrated that GLUT-4 expression in AT is 
crucial for systemic insulin-sensitivity [28, 29]. In line, GLUT-4 is considerably downregulated in diabetic-obese subjects, contributing to whole-body insulin resistance [30, 31].

Table 1.1: Main characteristics of adipose tissues in humans

\begin{tabular}{|c|c|c|c|c|}
\hline Type & Role & Localization & Histologic features & $\begin{array}{l}\text { Changes present in } \\
\text { dysfunctional AT }\end{array}$ \\
\hline $\begin{array}{l}\text { White adipose } \\
\text { tissue (WAT) }\end{array}$ & $\begin{array}{l}\text { Energy storage and } \\
\text { release } \\
\text { Thermo-protection } \\
\text { Endocrine organ }\end{array}$ & $\begin{array}{l}\text { Visceral (VAT) / } \\
\text { internal (intrathoracic } \\
\text { and intra-abdomino- } \\
\text { pelvic) } \\
\text { Subcutaneous (scAT) } \\
\text { - all over the body, } \\
\text { mainly buttocks, } \\
\text { thighs \& abdomen } \\
\text { Others: peri- or intra- } \\
\text { muscular, intra- } \\
\text { pancreatic, bone } \\
\text { marrow }\end{array}$ & $\begin{array}{l}\text { Large lipid laden } \\
\text { adipocytes (unilocular } \\
\text { lipid droplet) } \\
\frac{\text { Stromal vascular }}{\text { fraction (SVF): }} \\
\text { Pre-adipocytes } \\
\text { Macrophages } \\
\text { Fibroblasts } \\
\text { Endothelial cells } \\
\text { Hematopoietic cells }\end{array}$ & $\begin{array}{l}\text { Adipocyte } \\
\text { hypertrophy, impaired } \\
\text { lipid and glucose } \\
\text { metabolism, insulin } \\
\text { resistance } \\
\text { Macrophage } \\
\text { proliferation } \\
\text { and infiltration; shift in } \\
\text { M1/M2 ratio } \\
\text { Change in populations } \\
\text { of immune cells } \\
\text { Reduced pre- } \\
\text { adipocyte } \\
\text { differentiation, and } \\
\text { mitochondrial function }\end{array}$ \\
\hline $\begin{array}{l}\text { Brown adipose } \\
\text { tissue (BAT) }\end{array}$ & $\begin{array}{l}\text { Thermogenesis } \\
\text { Excess energy } \\
\text { dissipation? }\end{array}$ & $\begin{array}{l}\text { Neck } \\
\text { Interscapular } \\
\text { Supraclavicular } \\
\text { Paravertebral } \\
\text { Other? }\end{array}$ & $\begin{array}{l}\text { Small adipocytes } \\
\text { rich in mitochondria } \\
\text { (multilocular lipid } \\
\text { droplets) }\end{array}$ & $\begin{array}{l}\text { Decreased size } \\
\text { Whitening due to } \\
\text { vascular rarefaction } \\
\text { and mitochondrial } \\
\text { loss? * }\end{array}$ \\
\hline $\begin{array}{l}\text { Beige adipose } \\
\text { tissue (bAT) }\end{array}$ & $\begin{array}{l}\text { Thermogenesis } \\
\text { Excess energy } \\
\text { dissipation? }\end{array}$ & $\begin{array}{l}\text { Within the WAT } \\
\text { Supraclavicular } \\
\text { Other? }\end{array}$ & $\begin{array}{l}\text { Adipocytes rich in } \\
\text { mitochondria }\end{array}$ & $\begin{array}{l}\text { Transformation into } \\
\text { white adipocytes? }\end{array}$ \\
\hline
\end{tabular}

Moreover, the contribution of WAT to the 24-h energy expenditure (EE) of a healthy person is low $(\sim 5 \%)$ compared to the metabolically active fat free mass (SM and liver), which accounts for $\sim 70-80 \%$ of $24-\mathrm{h}$ EE [35]. Nevertheless, the importance of WAT for metabolic health is undeniable and also reflected by its function as a very active secretory organ (Table 1.2) [18].

\section{Histology}

AT is classified as loose connective tissue, which is composed of adipocytes that are surrounded by the extracellular matrix (ECM), rich in collagen fibers, and numerous capillaries. Embedded in the ECM is a heterogeneous population of non-adipocyte cells [36] like fibroblasts, pre-adipocytes, pericytes, endothelial and hematopoietic cells and adaptive and innate immune cells (e.g. macrophages, T cells, mast cells) that form the stromal vascular fraction (SVF) of AT (Table 1.1). Interestingly, there are 4-6 million SVF cells but only 1-2 million adipocytes per gram AT [18]. 
Healthy WAT is well vascularized, supplying the huge number of unilocular relatively large adipocytes (70-120 $\mu \mathrm{m}$ in diameter) adequately with oxygen and nutrients, showing minimal signs of local inflammation and fibrosis and preserved insulin sensitivity and mitochondrial function [1].

\section{Adipocytes and lipid droplets}

Adipocytes are specialized cells that store neutral lipids (TAGs) into lipid droplets (LDs), the most prominent intracellular organelle in white adipocytes occupying almost the complete cytoplasm. The diameter of the LDs can vary between 10 and $150 \mu \mathrm{m}$ in human WAT, which is the major determinant of fat cell size (volume). LDs, present both in AT and non-AT, contain a neutral lipid core (TAGs and cholesterol-esters) that is covered by a membrane rich in phosphatidylcholine and free cholesterol [37]. This phospholipid-monolayer has specific proteins inserted with the perilipin (PLIN) family being the most abundant [38]. Besides the PLINs, proteomics studies identified a huge panel of other LD-associated proteins, but not all their functions are clearly defined yet. However, their presence corroborates that LDs are interacting organelles balancing TAG storage and utilization $[39,40]$. The complex life cycle of cytosolic LDs has been suggested to start at the endoplasmic reticulum (ER), where the enzymes are located that finalize TAG synthesis [41]. The LD formation involves several lipid-intermediates (e.g. diacylglyerol) and proteins (e.g. seipin, PLIN-3), which also maintain the LDs integrity and regulate the fusion of small LDs (Fsp27 / Cidec in humans, [42]) to the large unilocular LD characteristic for human mature white adipocytes [43]. Degradation of LDs is achieved by hormonal regulated lipid hydrolysis, called intracellular lipolysis [44]. In adults, the number of adipocytes is notably constant throughout life, as it is set in childhood and not affected by the annual 10\% cell turnover (adipogenesis versus adipocyte death) [45]. Average TAG age is independent of adipocyte size when set in relation to body fat mass and they are renewed 6 times in 10 years (average age of a human adipocyte) [46]. However as recently described, TAG age in scAT is increased in overweight-obese subjects and the lipid turnover displays depot specific differences influenced by body fat distribution [47]. Taken together, the main role of LD containing adipocytes is packaging and managing of lipids, which enables the cells to buffer the influx of dietary lipids and sequester FAs [48], thus limiting their toxic effects (lipotoxicity).

\section{Adipocyte differentiation and lipid metabolism}

Adipogenesis is a tightly-regulated process, which transforms undifferentiated fibroblast-like preadipocytes to mature adipocytes. Pre-adipocytes are part of the adipose-derived stromal/stem cells, which can be isolated from the adipose SVF [49] and in-vitro differentiated towards adipocytes. 
The transcriptional cascade underlying adipocyte differentiation is regulated by several transcription factors with peroxisome proliferator-activated receptor (PPAR)-gamma [50] and CCAAT/enhancer binding protein (C/EBP)-alpha [51] being the "master" regulators. Furthermore, these transcription factors control lipid and glucose metabolism related gene expression in the maturing adipocyte, such as lipoprotein lipase (LPL) [52], fatty acid binding protein (FABP) 4 (murine aP2) [53], fatty acid synthase (FAS) [54], hormone sensitive lipase (HSL) [55] and GLUT4 [56]. Together, these genes influence the accumulation of lipids (lipogenesis) during adipogenesis (reviewed in [57]).

Lipogenesis comprises the uptake of free FAs (FFA) from the bloodstream, their conversion into neutral lipids and the packaging of those lipids in adipocytes' LDs. The FAs either originate from the diet or can be synthesized de novo from glucose in lipogenic tissues like the liver and human AT that also possesses the lipogenic enzymes for the FA synthesis [18], but its contribution to the TAG stores is minor [58 - 61]. TAGs are transported as TAG-rich lipoproteins (TRL) in the bloodstream. The chylomicrons $(75-450 \mathrm{~nm})$ are formed by the enterocytes and contain TAGs from the diet, whereas TAGs produced by the liver are packed in very-low-density lipoproteins (VLDL, 30-80 nm) [1]. Both TRLs are surrounded by a phospholipid monolayer, which contains specific apolipoproteins that enable their interaction with LPL e.g., on the surface of capillary endothelial cells. Adipocytes and myocytes synthesize LPL but it is exported to the capillary endothelium where it hydrolyzes TAGs of TRLs into FAs and 2-monoacylglycol, generating remnants that are further metabolized in the lipoprotein pathways (Figure 1.1).

LPL is a major player in postprandial lipid partitioning, because its activity is regulated tissuespecifically, dependent on metabolic circumstances e.g., nutritional state and physical activity, and under hormonal (insulin), apolipoprotein and angiopoietin-like protein control [62]. For example, in AT in the fasted state LPL is post-translationally inhibited by angiopoietin-like protein-4 [63] while in the skeletal muscle mRNA and total activity of LPL appear increased [62]. 


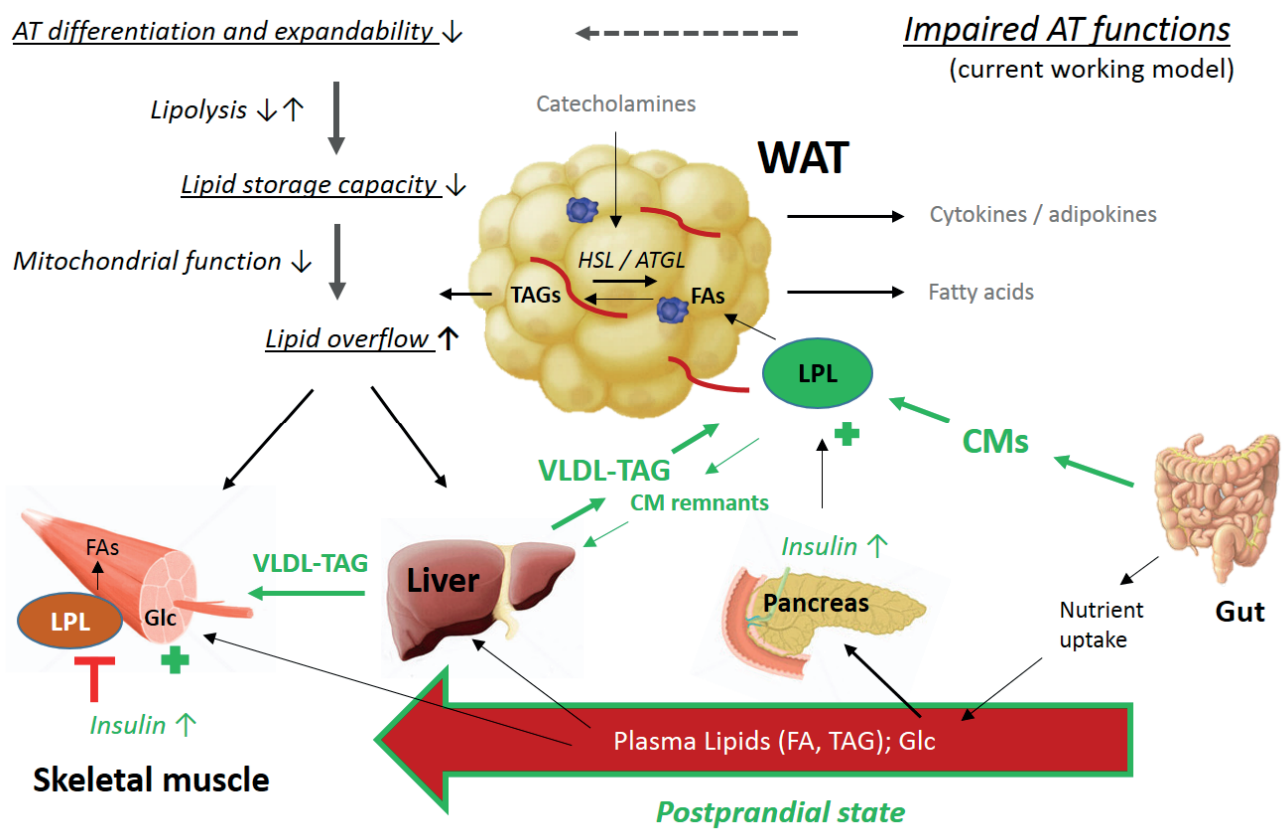

Figure 1.1: Inter-organ crosstalk and white adipose tissue (WAT) impairments

Healthy WAT stores FAs (delivered by lipoproteins) during energy surplus (postprandial state) and releases them during fasting (lipolysis). After a meal, substrates are distributed to the organs, with uptake into WAT being under the control of secreted insulin. Dietary TAGs reach WAT directly, incorporated in CMs, which enter the blood via the lymphatic system. Whereas, VLDLs transport liver-derived TAGs to the periphery. In addition, the endocrine organ WAT interacts with other tissues through the secretion of numerous molecules ("adipokines") and pro-inflammatory cytokines (see Tab. 1.2). These molecules guarantee proper metabolic responses of target tissues. E.g., in liver and SM, they improve insulin sensitivity and in the pancreas, they ameliorate survival and function of beta cells. In return, WAT is also influenced by hormones and factors released by its effector organs. Disturbances in AT functions, like reduced AT differentiation and expandability, decrease the storage capacity of WAT and can lead to lipid overflow, which subsequently causes ectopic fat storage (SM, liver, etc. see Fig. 1.2).

Abbreviations: ATGL - adipose triglyceride lipase; CM - chylomicron; FA -fatty acid; Glc - glucose; HSL - hormone sensitive lipase; LPL - lipoprotein lipase; SM - skeletal muscle; TAG - triacylglycerol; VLDL - very low-density lipoprotein. $\uparrow$ = increase; $\downarrow=$ decrease; + = stimulation; $\mathbf{T}=$ inhibition

Contrary, its activity is increased in AT and decreased in skeletal muscle and heart [64] after a meal, which is in line with the role of AT to buffer lipid fluxes in the postprandial period. The transport of LPL-derived and systemic FAs into and in adipocytes is protein-mediated and involves FA transport proteins (FATPs) [65], the human scavenger receptor CD36 and FABPs (e.g., FABP4) $[66,67]$. Before beta-oxidation or esterification to TAGs, phospholipids or second messengers, FAs are activated by the acyl-CoA synthetases [68] with ACS-1 being the major ACS-isoform in adipocytes. TAGs are mainly synthesized from glycerol-3-phosphate (G3P) and three activated acyl-CoA FAs (Kennedy pathway). The enzymes catalyzing the conversion of G3P into lysophosphatidic acid (lysoPA), lysoPA into phosphatidic acid (PA), and PA into diacyglycerol (DAG), are G3P acyltransferase (GPAT) 3, acylglycerol-3-phosphate O-acyltransferase 
(AGPAT) 2 and lipin 1, respectively. Alternatively, DAG can be derived from monoacylglycerol by the monoacylglycerol acyltransferase (MGAT; primarily occurring in intestine). In both pathways, DAG is finally condensed by diacylglycerol acyl transferase (DGAT) with another fatty acyl-CoA to a storable TAG molecule [69]. The most important hormonal factor influencing lipogenesis is insulin. Besides regulating LPL activity, it increases the uptake of glucose (required for G3P synthesis) and FAs into adipocytes via stimulation of GLUT-4 and FATP-1 translocation, respectively. Furthermore, it stimulates the transcription of lipogenic genes like FAS by regulating lipogenic transcription factors (e.g. ChREBP) [70]. Contrary growth hormone dramatically reduces lipogenesis during fasting [59] and stimulates lipolysis [71].

Lipolysis is the process of hydrolyzing TAGs into three FAs and one glycerol molecule. It occurs extracellularly by LPL to enable uptake of FAs from the blood and intracellularly to hydrolyze stored TAGs and release FAs for subsequent oxidation. The hormonal regulation (mainly by insulin, glucagon, catecholamines and natriuretic peptides (NPs)) of the TAG hydrolysis enables adipocytes to response to energetic needs by switching between lipid storage and lipolysis. NPs, which are released from the heart muscle and act via natriuretic peptide receptors (NPRs), are positive regulators of AT lipolysis, whereas catecholamines can affect lipolysis via the stimulating beta-adrenergic and inhibiting alpha-adrenergic receptors (G-protein coupled receptors), respectively. Hence, the net outcome of catecholamine-induced lipolysis depends on the balance between the lipolytic and anti-lipolytic adrenergic receptor stimulation [71]; however, lipolytic activity is also determined by major post-receptor events, including translocation and phosphorylation of proteins (see below).

The stepwise release of FAs from the glycerol backbone is achieved by three intracellular lipases working in a consecutive manner: adipose triglyceride lipase (ATGL), HSL [72] and monoacylglycerol lipase (MGL). In the low energy state (e.g. fasting), catecholamines elevate intracellular cyclic AMP (cAMP) via beta-adrenergic receptors, which activates protein kinase A (PKA)-dependent phosphorylation (P) of PLIN-1, the main LD-scaffolding protein in adipocytes [73]. In its un-phosphorylated state, PLIN-1 protects the lipids from hydrolysis by the intracellular lipases, whereas PLIN-1-P facilitates the maximal lipolytic response of ATGL and HSL [74], making the protein a crucial bi-functional modulator of lipid metabolism. ATGL is tightly associated to the LD and its activity is mainly regulated by proteins such as the inhibiting LDassociated protein Fsp27, G0S2/ABHD5 [75] and the activating CGI-58 cofactor [76]. The conversion of TAGs to DAG and FA is predominantly catalyzed by ATGL [77], which has a higher TAG specificity than HSL. In contrast to ATGL, HSL requires translocating to the LDs, which is initiated by phosphorylation of HSL via PKA [78]. PLIN-1-P induced conformational 
changes provide the lipases access to the LD and the active HSL-P can hydrolyze DAG to FAs and MAG ([71] and references therein). Studies in human adipocytes suggest that HSL is more important in catecholamine and NP-stimulated lipolysis than ATGL, but both lipases are pivotal in regulating the complete TAG hydrolysis under basal conditions [79]. The final step to complete lipolysis is catalyzed by the MGL, which is not under hormonal control [80]. Insulin is the major inhibitor of lipolysis and of FFAs efflux from adipocytes. It acts postprandial by stimulating the insulin receptor and successively decreases cAMP levels via the phosphatidyl-inositol 3-kinase (PI3K) pathway. Consequently, HSL is deactivated by de-phosphorylation and TAG synthesis (lipogenesis) is promoted by insulin (see above) [81]. ATGL activity is indirectly affected by insulin as the anti-lipolytic hormone activates phosphodiesterase $3 \mathrm{~B}$, which converts cAMP into 5'AMP and consequently decreases PKA activity [71], thus influencing the interaction between PLIN-1 and the ATGL activator CGI-58 [44]. In addition, insulin down-regulates mRNA of ATGL and induces the expression of its inhibitor G0S2 in human adipocytes [44, 82]. However, atrial NP, which activates the phosphorylation of PLIN-1 and HSL, and thus lipolysis, via NPR-A by a cGMP dependent pathways [83], is unresponsive to insulin's anti-lipolytic action [84].

Overall, AT is a complex organ with multiple functions, and its metabolic function is tightly controlled by hormones such as insulin (lipogenesis) and catecholamines (lipolysis), in response to external stimuli like exercise, diet, fasting or cold. Healthy AT stores TAGs and traps FA at an increased rate in the postprandial period (insulin-stimulated [48]) and can adjust the release of FA into the blood stream, when an energy deficit like fasting and exercise persists (catecholaminestimulated). Therefore, a well-tuned interplay of adipocyte differentiation, TAG synthesis, LD formation and lipolysis in adipocytes is of major importance for maintaining AT function, which prevents "lipid spill-over", hence avoiding ectopic lipid disposition in non-adipose tissues and related insulin resistance $[85,86]$.

\section{Endocrine organ function}

Since the identification of leptin in 1994 [87], WAT is accepted as secretory tissue that releases numerous metabolically and immunologically active substances (adipokines, cytokines, FAs, peptides), which act in an autocrine, paracrine or endocrine way causing pleiotropic effects such as modulation of whole-body insulin-sensitivity, energy balance and immune responses (Table $1.2 ;[88,89])$. Furthermore, they are involved in the development of numerous metabolic complications like insulin resistance [90], CVD [91] and the chronic low-grade inflammation associated with obesity [92]. Some molecules are primarily secreted within the AT by adipocytes 
(adiponectin, leptin, visfatin), whereas others are preferentially produced by cells in the SVF, especially macrophages (monocyte chemoattractant protein (MCP)-1/CCL2, interleukin (IL)-6, tumor necrosis factor (TNF)-alpha) [24]. However, the exact contribution of all different cell types in AT to its secretion profile is still under evaluation. The secretory profile of adipocytes depends on their differentiation state [93] and large adipocytes are characterized by an altered secretion pattern, which might promote AT inflammation [94]. Besides its negative impact on insulin sensitivity (see below), AT inflammation has been suggested to be a prerequisite for proper AT plasticity and remodeling, and is therefore of major importance for normal tissue homeostasis [95]. Animal and human studies demonstrated that AT harbors a number of innate and adaptive immune cells. Innate macrophages are tissue-resident phagocytes and scavenge cell debris of apoptotic cells and regulate angiogenesis and ECM re-modulation [96]. Alternatively, activated M2macrophages, regulatory T-cells and eosinophils contribute to the production of anti-inflammatory cytokines e.g., IL-10 in lean AT and are involved in the maintenance of insulin sensitivity [97]. Conversely, classically activated M1-macrophages, derived from monocytes of the blood stream, secrete pro-inflammatory cytokines such as TNF-alpha and IL-6 but are often lower in number in lean AT ( 10\% [98]). VAT is more prone to macrophage infiltration than subcutaneous AT and this is paralleled by increased pro-inflammatory secretion [99]. During body fat mass expansion, the number of immune cells in AT increases $[32,97]$, whereas weight loss can partly reverse such changes in scAT of obese subjects [100]. The best-characterized adipokines are adiponectin and leptin. Adiponectin is secreted in the form of distinct polymer-complexes, which display antiinflammatory, anti-atherogenic and insulin sensitizing effects in peripheral organs $[101,102]$ via adiponectin receptors [103]. Compared to adiponectin, leptin plasma levels are much lower (2-8 $\mu \mathrm{g} / \mathrm{ml}$ vs $5-30 \mathrm{mg} / \mathrm{ml}$ ) and its secretion from WAT increases with fat accumulation and decreases with fat loss, reflecting its role as a signal of adipocyte energy storage. The leptin receptor is expressed in nearly all peripheral and insulin sensitive tissues. Its main metabolic functions, which partly overlap with the ones of adiponectin, are the regulation of the energy balance, affecting glucose and lipid metabolism (improved insulin sensitivity) and modulating angiogenesis (reviewed in $[88,107])$. In human obesity, elevated leptin plasma levels are reported and often accompanied by leptin resistance in the brain and periphery [108], whereas levels of the insulin sensitizer adiponectin are often decreased [109]. Furthermore, MCP-1, interleukins, PAI-1, TNFalpha [89], adipsin [104], and apelin [110] secretion affects a wide range of processes related to energy metabolism and immune response (Table 1.2) and their secretion is dependent on adipocyte size and AT depot [24]. 
Table 1.2: Molecules secreted by white adipose tissue and their metabolic role

\begin{tabular}{ll}
\hline Secreted molecule & Role in... / Effect on... \\
\hline Adipokines & \\
\hline Adiponectin & FA oxidation, hepatic glucose synthesis, (anti-) inflammation, insulin sensitivity \\
\hline Adipsin & immune-stress response, lipid metabolism, insulin secretion and resistance \\
\hline Apelin & energy metabolism, insulin sensitivity, angiogenesis \\
\hline Leptin & appetite control, (pro-) inflammation, lipid metabolism, insulin secretion and resistance \\
\hline Visfatin & NAD+ synthesis, insulin signaling, (pro-) inflammation \\
\hline & \\
\hline Cytokines/Chemokines & (pro-) inflammation, insulin resistance, energy metabolism \\
\hline Interleukin (IL)-1-beta & (pro- and anti-) inflammation, insulin resistance, liver substrate metabolism \\
\hline IL-6 & (pro-) inflammation, insulin resistance \\
\hline IL-8 (CXCL8) & (anti-) inflammation, insulin sensitivity \\
\hline IL-10 & stimulation of macrophage recruitment, (pro-) inflammation \\
\hline Monocyte chemoattractant & \\
protein (MCP)-1 (CCL2) & (pro-) inflammation, insulin resistance \\
\hline $\begin{array}{l}\text { Tumor necrosis factor (TNF)- } \\
\text { alpha }\end{array}$
\end{tabular}

\begin{tabular}{ll}
\hline Other factors & \\
\hline Hepatic growth factor (HGF) & angiogenesis, (pro-) inflammation \\
\hline $\begin{array}{l}\text { Plasminogen activator inhibitor } \\
\text { (PAl)-1 }\end{array}$ & (pro-) inflammation, insulin resistance, vascular homeostasis, metabolism of ECM \\
\hline Retinol binding protein (RBP)-4 & lipid and glucose metabolism, insulin resistance, chemoattraction of immune cells
\end{tabular}

Expression and secretion of cyto-/adipokines/factors is different in AT depots and affected by body weight, adipocyte size and gender. Key references: [1, 17, 24, 33, 36, 88, 89, 95, 104-106]. This list is not comprehensive.

\section{AT expandability}

Energy intake and expenditure (basal metabolic rate plus physical activity) determine the energy balance. A constant positive energy balance leads to an expansion of WAT and a concomitant remodeling in the tissue cellularity, ECM and blood vessels $[111,112]$. AT expansion allows storage of excess energy in form of fat via: (1) hypertrophy of adipocytes (increase of cell size) and/or (2) hyperplasia of proliferating pre-adipocytes into differentiated adipocytes (increase of cell number, see above) $[45,113]$. Thus, AT is a metabolic sink and its expansion, which is often impaired in metabolically compromised individuals ("AT expandability hypothesis"), protects the human body from harmfully elevated concentrations of plasma TAGs and FAs mainly in the postprandial period [114]. Although, it was reported that the adipocyte number is rather constant during adulthood independent of weight fluctuations [45], earlier studies suggest that hyperplasia can be an adaptive response to weight gain in adults [115]. Both, hypertrophy, and hyperplasia occur sex-, fat depot- and fat cell size-dependent [116]. With regard to healthy WAT expansion, gluteofemoral fat ('peer shape') plays a key role, as it traps TAGs for the long term, protecting the body from FA overflow and ectopic fat disposition, and is associated with a beneficial adipokine profile and metabolic health [117]. In contrast, abdominal subcutaneous fat accumulation, 
probably due to dysfunctional peripheral scAT, reaching its expansion limit, and increased visceral fat accumulation ('apple shape'), is associated with increased metabolic risks [118]. This correlates with a more pro-inflammatory cytokine secretion (PAI-1, IL-6, TNF-alpha; [89]) from hypertrophic adipocytes and therefore contributes to inflammation and impaired metabolic sensitivity to hormones, making them less favorable (unhealthy) storage depots $[24,118]$.

\section{Adipose tissue dysfunctions and its metabolic implications}

WAT dysfunction rather than fat mass per se seems to be an early perturbation in the emergence of human obesity [32] and is often associated with insulin resistance. The current concept underlying the development and effects of dysfunctional AT is the following [2]: The inability of scAT to expand through hyperplasia (decreased adipogenesis), coupled with impaired angiogenesis, generates on the one hand hypertrophic adipocytes $(>150 \mu \mathrm{m})$ and a relative oxygen deficit (hypoxia) $[119,120]$, leading to impaired glucose and lipid metabolism (insulin resistant) [121], changes in oxidative and ER stress and a pro-inflammatory secretion profile (e.g. increased TNF-alpha, IL-6, decreased adiponectin) [94, 122]. On the other hand, this promotes pro-fibrotic conditions [123] due to disturbances in ECM remodeling and reducing AT plasticity [111], which might further compromise adipogenesis. Because the storage capacity of enlarged adipocytes is limited, cells might become necrotic, causing the infiltration of additional immune cells and subsequent local inflammation [124, 125]. Lipid spill-over (overflow) and subsequent ectopic LD accumulation to entrap excess lipid intermediates/metabolites (diacylglycerols and ceramides) in the liver, skeletal muscle, pancreas, and visceral depots can trigger local and systemic insulin resistance (lipotoxicity) [126] (Figure 1.2) [2]

Impaired TAG turnover [127] marks hypertrophic AT and thus poses a risk for further fat gain and obesity-induced metabolic disturbances $[128,129]$. In obese and insulin resistant subjects, LPL activity [130] and subsequent FA uptake via CD36 and other transporters, are decreased, thus promoting lipid overflow into the circulation [86]. In addition, adipocyte lipolysis is impaired in obesity due to reduced cAMP signaling [46]. Fasting lipolysis expressed per unit fat mass [131] and ATGL and HSL expression is reduced in insulin-resistant obesity [132], although the wholebody basal lipolysis might be elevated because of the increased total fat mass (mass effect). On the other hand, whole-body lipolysis is elevated in the postprandial state in obese individuals, due to an attenuation of the insulin-mediated suppression of lipolysis in AT [86] and skeletal muscle [133]. In-vitro and in-vivo studies also showed that catecholamine- and NP-induced lipolysis is blunted in scAT of obese and T2DM subjects [134, 135]. This blunted lipolytic response may 
contribute to a decreased TAG turnover, an increased AT mass [46] and a reduced lipid-buffering capacity resulting in lipid spill-over.

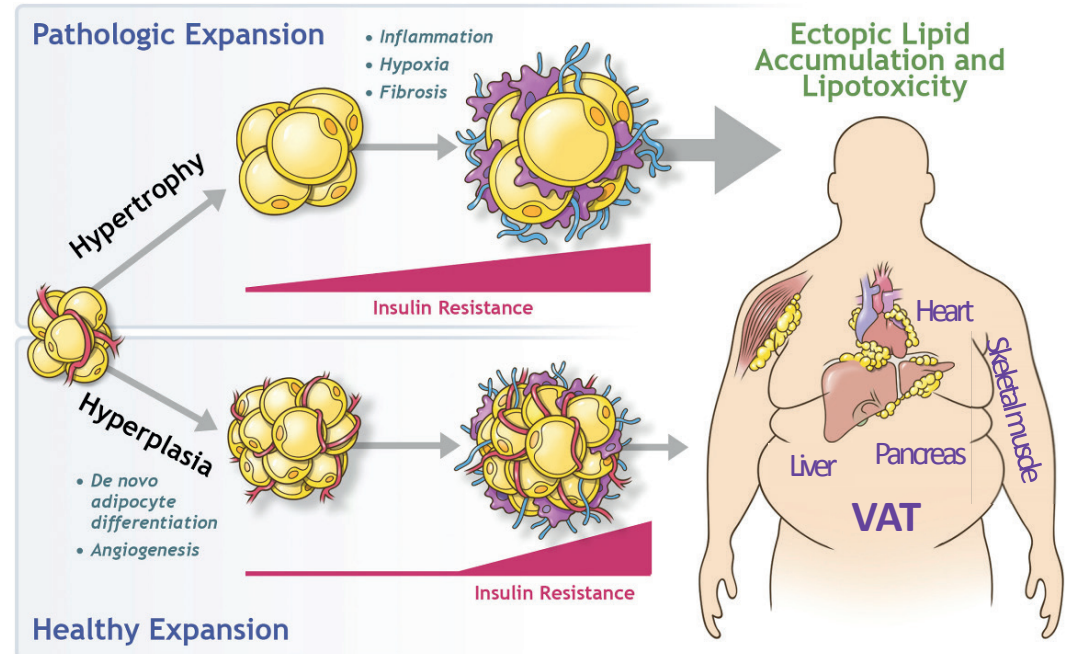

\section{Figure 1.2: Mechanisms of AT expandability preceding obesity}

Chronic caloric excess leads to expansion of WAT by an enlargement of existing adipocytes (hypertrophy) and/or an increase in adipocyte number (hyperplasia) via pre-adipocyte differentiation. Pathologic expansion through hypertrophy is associated with inflammation, hypoxia, and fibrosis, with early onset of insulin resistance. Adipocyte dysfunction leads to detrimental lipid overflow and their disposal in VAT and non-adipose organs. Healthy expansion through hyperplasia occurs concomitantly with angiogenesis and prevents or delays the onset of both insulin resistance and ectopic lipid accumulation. (figure adapted from [2])

Hence, an alternative path of lipid breakdown, autophagy, might become active under such pathophysiological conditions [32]. In line, markers of autophagy were elevated especially in VAT of obese subjects and correlated with the degree of obesity and fat cell hypertrophy [136, 137]. Although the autophagic clearance seems attenuated in subcutaneous adipocytes of obese subjects [138]. So far, largely studied in hepatocytes and animals, the contribution of this alternative pathway to lipid breakdown and adipocyte function needs to be confirmed in human adipose tissue [138]. Additionally, a disturbed LD storage capacity [139] may play a role in the development of AT dysfunction and might be related to altered expression of lipid storage promoting LDassociated proteins (e.g. PLIN-1, CIDEC), although data in mice and humans are conflicting [8, $71]$.

Mitochondria provide energy by oxidation of different substrates depending on the nutritional status. In times of constant overfeeding mitochondria of various tissues may fail in adapting the substrate selection (metabolic inflexibility) [140]. Particularly lipid-overload may increase ROS formation and the production of toxic lipid species, which contribute besides ER-stress and proinflammatory processes to mitochondrial dysfunction in WAT [141]. Such impaired metabolic 
flexibility and mitochondrial function have been implicated in the accumulation of lipids and related insulin resistance in skeletal muscle of obese T2DM subjects [86]. A study by Yin et al. reported that the mitochondrial function (oxygen consumption and citrate synthase) in adipocytes of obese subjects is also attenuated [142]. This might partly be attributable to impaired lipolysis as demonstrated in a recent in-vitro study using differentiated human adipocytes. It indicated that adipose lipase deficiency contributes to mitochondrial dysfunction, lipid accumulation and insulin resistance, in a PPAR-alpha-dependent manner [143]. This confirms previous findings made in cardiac muscle of lipase-deficient mice [144]

It is now well established that hypertrophic AT of obese individuals is infiltrated by macrophages [98]. The concomitant histological changes in AT during obesity seem to be triggered by dysfunctional adipocytes and are characterized by an accumulation of M1-macrophages (crownlike structure around metabolically deficient and dying cells, which is more frequent in VAT [124]) and a change in the M1/M2 macrophage ratio [145] leading to an increased pro-inflammatory cytokine and chemokine secretion [146]. In addition, other immune cells (mast cells, neutrophils, B- and T-lymphocytes) infiltrating WAT might also contribute to this pro-inflammatory shift [147]. The presence and secretion of mediators (e.g., IL-1-beta, IL-6, MCP-1 and TNF-alpha) by recruited immune cells influence themselves the function of adipocytes by changing their secretion profile (increased MCP-1, IL-6 and CXCL8/IL-8; decreased adiponectin) [148]), which further enhances the inflammation process, inducing insulin resistance [145] and inhibiting pre-adipocyte differentiation [149]. These alterations are most likely mediated by lipids, ROS, or microbiotaderived lipopolysaccharides (LPS) [150]. Possible mechanisms explaining the effects of these mediators and hence the development of obesity-associated inflammation include the activation of the innate immune receptors (e.g., TLR-4) and the inflammasomes (e.g., NLRP3) in WAT, which regulate the production of inflammatory cytokines (e.g., IL-1-beta and IL-6), besides others such as increased ER-stress and cell death $[145,150]$. In the last decade, numerous studies investigating cytokines involved in obesity-induced AT inflammation have been performed. They showed that other interleukins such as IL-17 [151] or additional members of the IL-1 family play important roles in WAT biology. Overall, many different factors are contributing to AT inflammation that may be considered as an adaptation for a healthy WAT expansion [152] but eventually could cause the systemic low-grade inflammation found in obesity $[95,147]$.

\section{Metabolic consequences of AT dysfunction}

Collectively, AT dysfunction can be described by impaired adipogenesis, hypertrophic adipocytes, visceral fat accumulation, inflammation, and disturbances in lipid and glucose metabolism and is not caused by increased body fat mass per se. It is an early or even primary event in human obesity 
and implicates metabolic consequences, which are related to impaired health [32]. Body fat distribution and depot-differences between scAT and VAT, e.g., reaction to hormonal stimulation, further determine the extent of health impairments. Insulin resistant hypertrophic scAT seems to redirect lipids when its storage capacity is exceeded [114]. A higher lipid efflux (overflow) from impaired scAT enhances VAT expansion and ectopic fat storage, which is associated with metabolic syndrome, CVD, and peripheral and hepatic insulin resistance [32]. Decreased insulin sensitivity and increased lipolytic activity in VAT and its anatomical location may contribute to an elevated influx of FAs and cytokines into the liver [153]. This "portal hypothesis" and increased circulating lipids coming from dysfunctional scAT, may therefore increase hepatic lipid accumulation [154, 155] and alter VLDL-TAG release in the postprandial state [156], subsequently shunting lipids to skeletal muscles. There, increased uptake and accumulation disrupts muscle functions (lipid handling) and insulin sensitivity [157, 158], which might expedite whole-body insulin resistance [159].

The augmented immune cell infiltration in WAT and the chronic low-grade inflammation in subjects with nascent metabolic syndrome indicate a close relationship between the immune system and obesity. Thus, an accompanying overproduction of pro-inflammatory cytokines such as IL-1-beta, IL-6 and 8, TNF-alpha, RBP-4 and PAI-1 [160], whose actions are mediated by the stimulation of stress-induced pathways [8] and associated low levels of the insulin-sensitizer adiponectin [161], further enhance systemic insulin resistance [95].

In addition, reduced expression/translocation of GLUT-4 [128] and diminished postprandial VLDL-TAG clearance and buffering of FFA by AT [162], observed in insulin resistant and obese subjects, seem to contribute to hyperglycemia [163], hyperlipidemia and compensatory hyperinsulinemia [162]. These, together with insulin resistance and chronic low-grade inflammation, promote the development of obesity-related chronic metabolic diseases, including T2DM, CVD and NAFLD [158]. Hence, a comprehensive understanding of the manifestation of AT dysfunction, which may be the differentiating factor between obesity sub-phenotypes (metabolically healthy versus metabolically unhealthy obese subjects [164]), could provide novel and improved treatments for metabolically unhealthy obesity, but requires valid models.

\section{Models to study AT lipid metabolism and function}

Rodent, in-vitro and in-vivo models (e.g., diet-induced obese C57BL/6 mice) are the basis for our current understanding of AT (dys)-function. In the last 50 years many rodent in-vitro models were established to study the differentiation of pre-adipocytes into adipocytes, using e.g., the cell lines 3T3-L1 [165] and C3H10 T1/2 [166] or primary adipocyte progenitor cells isolated from the SVF [167]. However, human primary adipose-derived stem/stromal cells (ASC) also called multipotent 
adipose-derived stem (hMADS) cells [168], isolated and selected pre-adipocytes from the SVF and mesenchymal stem cells (MSCs) from bone-marrow represent validated human models for the in-vitro study of adipocyte function and metabolism [169]. Unlike the human cell strain SGBS [170] and the LiSa-2 cell line, ASCs are a heterogenic cell population retaining their distinct donorand depot-specific metabolic profiles [23, 169], making them excellent candidates for studying intrinsic disturbance in adipocyte function. More recently Todorcevi et al. [171] generated immortalised human pre-adipocytes of paired subcutaneous abdominal and gluteal WAT depots. These cell lines retained depot-specific gene expression patterns and enable to study in parallel pre-adipocytes from more than one depot, thus helping to investigate depot differences that may contribute to the metabolic risk-determining body fat distribution [171]. Additionally, co-cultures [172] and 3D models [173] with human cells are being established to investigate the various metabolic interactions between adipose and other tissues. Nevertheless, ASCs derived from wellcharacterized lean and obese donors are the ideal model to study AT biology, whereas studying energy balance in-vivo still requires human subjects.

In summary, healthy scAT buffers the daily lipid fluxes and minimizes ectopic lipid storage. If scAT becomes dysfunctional and its expandability gets limited, an increase of VAT mass and ectopic fat disposition may follow, disturbing peripheral glucose utilization, promoting insulin resistance and systemic low-grade inflammation, which are associated with increased T2DM and CVD risk. Therefore, preventing obesity-associated (sc)AT dysfunction by enhancing its fat buffering ability, differentiation capacity, insulin sensitivity and subsequent uptake of glucose, or redistributing lipids into more beneficial depots, is pivotal to protect from obesity-related metabolic impairments. Nutritional bio-actives are one of the potential therapeutic options to prevent or treat AT dysfunction in human metabolic diseases.

\section{Nutritional bio-actives and AT (dys)-functions}

AT dysfunction seems to be the major driver of obesity-related metabolic derangements [174]. In the last decades, numerous studies have demonstrated that plant-derived polyphenols and carotenoids, and polyunsaturated fatty acids (PUFAs) from fish and algae are potent anti-oxidant and anti-inflammatory nutrients. They influence adipocyte function in different cell and animal models of obesity [175], thereby providing potential beneficial effects in modulating obesityrelated metabolic disturbances in both rodents and humans (for review see [17, 86, 176-178]). Therefore, improving AT function with nutritional bio-actives, by increasing AT lipid buffering 
capacity and subsequently reducing the inflammatory response, seems a strategy of clinical importance besides life-style changes including increased physical activity [179]. However, the physiological complexities detailed above and varying treatment doses, periods and study designs require a careful interpretation of the results that are currently available in the scientific literature. The following section sketches the effects of the carotenoid lycopene, the polyphenols resveratrol and (-)-epigallocatechin-3-gallate and the n-3 PUFA eicosapentaenoic acid, on markers of AT (dys)-function and related health benefits. For the purpose of this thesis, metabolic profiles are defined as measurements of the multiparametric metabolic response (including parameters like mRNA, proteins and lipids) of living systems (cell models and human subjects) stimulated by single or combinations of bio-actives with partly distinct mechanisms of action [180].

\section{Carotenoids}

Carotenoids are naturally occurring pigments in plants and photosynthetic bacteria and serve a large number of functions in nature. They play a role as macular pigments, precursors of vitamin A and act as antioxidants [181] and probably as adipose biology modulators [182, 183]. Carotenoids have also been associated with numerous health effects: decreased risk of macular degeneration, cancers and CVDs $[184,185]$. Beta-carotene, alpha-carotene, beta-cryptoxanthin, lutein, zeaxanthin, and lycopene (Lyc) are the most abundant carotenoids in human plasma and account for $\sim 70 \%$ of all carotenoids identified in human plasma and tissues. Carotenoids must be taken up from the diet (e.g., tomato, carrots and spinach) and are divided into two major groups: xanthophylls, which are oxygenated carotenoids (lutein and zeaxanthin) and carotenes, which are hydrocarbon carotenoids that are either cyclized (beta-carotene) or linear (Lyc) [186]. Betacarotene is the best-known carotenoid since it is the main precursor of vitamin A (retinol), an essential molecule for vision and many gene-regulating processes as reviewed elsewhere [176, 187]. AT is an important storage depot for the lipid-soluble carotenoids and they are found in adipocytes mainly in the TAG core of the LD and associated to plasma membranes [188].

The anti-inflammatory and antioxidant properties of carotenoids and carotenoid-derivatives make them predestined to affect the inflammatory and secretory profile of AT. Carotenoids and their conversion products interact with nuclear receptors and inflammatory pathways in adipocytes and AT macrophages, thereby affecting adipogenic and lipogenic gene expression (reduced adipogenesis and increased lipid turnover), reducing oxidative stress (also by scavenging ROS directly) and modulating the expression of specific adipokines and inflammatory cytokines, respectively [176, 189]. Several epidemiological studies in overweight and obese adults and children report an association of low serum carotenoid levels with risk factors of the metabolic syndrome e.g. increased HOMA-IR and inflammatory markers in plasma, independent of obesity- 
related measures, but an inverse association between carotenoid plasma levels and adiposity. Hence, fat mass appears to sequester carotenoids and thus may control blood levels as recently suggested for beta-carotene [190] or reflect a higher consumption in obese AT [183]. In line, the few intervention studies testing carotenoid supplementation on adiposity showed consistently that a higher intake of carotenoids resulted in increased adiponectin levels and decreased body fat (see [183] and references therein) and insulin resistance [191]. In summary, because carotenoid levels are reduced in blood and adipocytes (beta-carotene) in human obesity [190], their supplementation seems advantageous to ameliorate WAT lipid turnover and to scavenge ROS, thereby possibly affecting AT inflammation and insulin sensitivity.

Lycopene is the most abundant carotenoid present in tomatoes and one of the main carotenoids in the human diet [192]. The acyclic Lyc (Table 1.3) has the same molecular mass and chemical formula than beta-carotene and is the second most abundant carotenoid in human AT [193]. Its absorption, transport, metabolism, and biological activities are rather ill-defined compared to betacarotene [194]. Lyc is well known for its antioxidant capacity, its anti-carcinogenic (prostate) and cardioprotective effects $[185,195]$. It also reduces oxidative DNA damage in healthy volunteers [196]. Furthermore, evidence is accumulating from epidemiological, animal and intervention studies for its anti-inflammatory and anti-diabetic properties in relation to chronic diseases accompanying obesity [197-199]. It has been shown that lycopene and its metabolite apo-10'lycopenoic acid inhibit TNF-alpha-induced pro-inflammatory cytokine and chemokine expression in murine and human adipocytes [200, 201]. In addition, treatment of rodents with Lyc or a Lycenriched tomato product has shown to improve gene expression and secretion of adipokines (increase of adiponectin) and cytokines (decrease of MCP-1 and IL-6) in AT and liver, either without affecting AT mass (obese rats) $[202,203]$ or with reducing HFD-induced adiposity and HOMA-IR (mice) [204]. Intervention studies in healthy and moderately overweight subjects and metabolic syndrome patients demonstrated the beneficial effects of tomato juice and Lyc supplement / Lyc rich-diet regarding metabolic risk reduction in humans. Li et al. reported that daily tomato juice consumption (32.5 mg Lyc/d, 8 weeks) induced reductions in waist circumference, serum levels of cholesterol and the inflammatory MCP-1, and an increase in serum levels of the anti-inflammatory adiponectin in young healthy Taiwanese women [208]. However, these changes were not accompanied by a decreased body fat content nor an improved insulin sensitivity. Furthermore, McEneny and colleagues showed, that Lyc supplementation $(70 \mathrm{mg} / \mathrm{d})$ and a diet enriched in Lyc (224-350 mg/d) for 12 weeks reduced systemic inflammation and 
ameliorated HDL functionality in moderately overweight men and women, which implies a cardioprotective effect [209].

Table 1.3: Structure and demonstrated effects of dietary bio-actives on adipose tissue

\begin{tabular}{|c|c|}
\hline Structure (bio-actives) & Effect on... \\
\hline \multicolumn{2}{|l|}{ (all-E)-lycopene } \\
\hline $\mathrm{H}_{3} \mathrm{C}$ & $\begin{array}{l}\text { - Gene expression in AT } \\
\rightarrow \text { mRNA of PPAR-gamma, SIRT-1, CD36 } \\
\text { - Cyto-/adipokine secretion } \\
\rightarrow \text { adiponectin, leptin mRNA levels; MCP-1, IL-6 secretion } \\
\text { - Oxidative stress } \\
\text { - AT distribution }\end{array}$ \\
\hline \multicolumn{2}{|l|}{ trans-resveratrol } \\
\hline $\mathrm{OH}$ & $\begin{array}{l}\text { - Differentiation of pre-adipocytes } \\
\rightarrow \text { mRNA of PPAR-gamma and CEBP-alpha } \\
\text { - Lipogenesis (WAT) } \\
\rightarrow \text { FAS activity, FAS mRNA levels, ACC activity } \\
\text { - Lipid uptake and lipolysis } \\
\rightarrow \text { Lipases expression; LPL, ATGL, HSL mRNA levels } \\
\text { - Apoptosis } \\
\text { - Mitochondrial function } \\
\rightarrow \text { Heat production (BAT), UCP-1 activity, FFA oxidation } \\
\text { - Cyto-/adipokine secretion } \\
\rightarrow \text { PAI-1, MCP-1, interleukins, TNF-alpha } \\
\text { - Energy expenditure, AT weight } \\
\text { - Insulin sensitivity }\end{array}$ \\
\hline \multicolumn{2}{|l|}{ EGCG } \\
\hline & $\begin{array}{l}\text { - Differentiation of pre-adipocytes } \\
\rightarrow \text { mRNA of PPAR-gamma and FAS } \\
\text { - Lipolysis } \\
\text { - Cyto-/adipokine secretion } \\
\rightarrow \text { adiponectin, TNF-alpha, IL-6 } \\
\text { - Energy expenditure } \\
\text { - Fat oxidation } \\
\text { - Insulin sensitivity } \\
\text { - Body weight, fat mass }\end{array}$ \\
\hline \multicolumn{2}{|l|}{ n-3 PUFA (EPA) } \\
\hline & $\begin{array}{l}\text { - Differentiation of pre-adipocytes } \\
\rightarrow \text { mRNA of PPAR-gamma } \\
\text { - Cyto-/adipokine secretion } \\
\rightarrow \text { adiponectin, leptin, MCP-1, IL-6, CRP } \\
\text { - Inflammation } \\
\rightarrow \text { NF-kappaB pathway; macrophage infiltration } \\
\rightarrow \text { prostaglandins/leukotrienes synthesis } \\
\text { - Mitochondrial biogenesis / browning } \\
\rightarrow \text { beta-oxidatio } n\end{array}$ \\
\hline
\end{tabular}

Effects might depend on cell/animal model, AT depots and could be affected by body weight, metabolic donor characteristics and gender. Key references: [205-207]. This list is not comprehensive. 
Men and women with metabolic syndrome showed a significant attenuation of inflammation and endothelial dysfunction accompanied by improved insulin sensitivity after tomato-juice consumption (4 times / week for 8 weeks) [210].

In summary, lycopene seems to be a promising nutritional bio-active for attenuating inflammatory processes occurring in dysfunctional AT. However, it remains unclear whether these changes also translate into beneficial metabolic effects on the long-term in human.

\section{Polyphenols}

Polyphenols can be divided in 5 major classes: stilbenes, flavonoids, lignans, phenolic acids and other polyphenols, which are further sub-classified and often found as glycosides in plants. They have been identified as antioxidant ingredients in the diet decades ago and have recently been studied for their anti-diabetic (caffeic acid), anti-obesity (curcumin), blood glucose-lowering (phloridzin) and phytoestrogenic (genisitin, lignans) properties [211], secondary to their gut microbiota modulating (quercetin) [212] and anti-cancerogenic effects [213]. The most recognized and abundantly available dietary polyphenols are epigallocatechin-3-gallate (EGCG, flavonoid) and trans-resveratrol (Res, stilbene) (Table 1.3), found in tea (green, oolong) and hazelnuts or in grapes (red wine) and peanuts, respectively (Phenol-Explorer DB, [214]). Both entered the nutritional "stage" because their consumption in form of green tea (Japanese population) [215, 216] or red wine ("French Paradox") [217] was inversely associated with cardiometabolic disease in humans. Ever since, they have been extensively studied for their promising health-promoting (anti-obesity and anti-inflammatory) effects as reviewed elsewhere [177].

The main catechin in green tea, $\boldsymbol{E} \boldsymbol{G C G}$, has consistently been shown to inhibit adipogenesis in murine adipocytes by downregulating PPAR-gamma and FAS [218] and pre-adipocyte proliferation by reduced activation of the ERK/Cdk2 signaling pathway [219]. Additionally, it was also shown that green tea catechins enhance (catecholamine-induced) lipolysis in 3T3-L1 adipocytes [220]. The potential of EGCG to reduce lipid accumulation and alleviate diabetes risk has further been demonstrated in-vivo in obese rodents [221, 222]. The treatment with EGCG reduced AT mass, increased fat oxidation (decreased RQ), modified related gene expression [223] and decreased nutrient absorption [224], possibly by activating AMP kinase (AMPK) and inhibiting intestinal enzymes/transporters [225], leading to reduced body weight and improved glucose homeostasis and insulin sensitivity [226, 227]. A recent study by Bao et al. corroborated the anti-inflammatory effect of EGCG. They showed that long-term supplementation alleviated 
inflammation by reducing macrophage infiltration into AT and suppressing TNF-alpha and IL-6 expression in HFD-rats [228].

In humans, EGCG and green tea extract (GTE) supplementation depicts so far inconsistent data regarding their effects on body composition, whole-body and peripheral insulin sensitivity. Hsu and co-workers reported that the intake of GTE ( $\sim 300 \mathrm{mg} / \mathrm{d}$ or $\sim 860 \mathrm{mg} / \mathrm{d}$ EGCG) for 12 weeks did not affect body weight or waist circumference in obese Asian women compared to PLA, although a reduction in plasma lipids (total and LDL-cholesterol) was observed with the higher GTE dosage [229, 230]. Furthermore, in Caucasian healthy adults [231] and in premenopausal obese women on an energy-restricted diet [232] no (additional) effect on body weight, EE, fat oxidation, and cardiometabolic risk factors was found after 12-week EGCG supplementation (300-600 mg/d). Interestingly, short-term studies with EGCG reported an increase in fat oxidation and in some cases an increased EE in normal and overweight men [233-235]. Similar controversial findings were observed for the effects of EGCG on insulin sensitivity. While a recent study in obese hypertensive adults ( 3 months GTE) showed an improvement in insulin levels and lipid profile [236] other clinical trials could not find an effect on insulin sensitivity in obese males (e.g., [237], for further references see reviews [225, 238-240]). In conclusion, the current data suggest that the effects of EGCG seem to be influenced by gender, metabolic status (BMI), dosing and duration of the supplementation [241]. However, this anti-inflammatory catechin might favorably alter dysfunctional processes (in AT and skeletal muscle) underlying obesity-related metabolic complications by mutual interaction with its human target enzyme catechol-O-methyl-transferase [242, 243] and by affecting AMPK activity [238]. Although, overall EGCG seems to have no clinically significant impact on body fat reduction [244].

Trans-resveratrol (3,4,5-trihydroxystilbene), a phytochemical in the skin of red grapes with broad bio-active properties (e.g., cardio-protective and anti-cancerogenic; for review see [245]), has been recently investigated for its potential caloric-restriction like effects against obesity and related metabolic diseases [246, 247]. Numerous studies using adipocytes have shown that Res has antiobesity properties by suppressing pre-adipocyte proliferation and differentiation [248], decreasing lipogenesis and promoting hormone-induced lipolysis [249] and beta-oxidation [250] in white adipocytes. These effects may be mediated by the activation of the central regulators of energy metabolism, AMPK [251] and NAD-dependent deacetylase sirtuin-1 (SIRT1) [252], which subsequently engages PPAR-gamma coactivator (PGC)-1-alpha [253], a regulator of mitochondrial biogenesis [254]. A reduced lipid accumulation and a concomitant suppression of adipogenic (PPAR-gamma, C/EBP-alpha) [255] and lipogenic (FAS, LPL) [256] genes 
accompanied the inhibition of adipogenesis and lipogenesis. Whereas the stimulation of lipolysis and beta-oxidation in adipocytes by Res can be partly attributed to elevated ATGL mRNA and protein levels [257] and reduced malonyl-CoA (inhibitor of beta-oxidation) levels via reduction of ACC activity [258], respectively. Resveratrol prevented the gain of body weight and AT mass and the emergence of insulin resistance $[253,259,260]$ in diet-induced obese rodents. This was accompanied by a diminished adipogenesis and amelioration of obesity-related chronic inflammation in VAT and improved plasma lipid profiles, adipokine levels and fat oxidation [251, 259]. Besides the improvements of white AT function and glucose homeostasis [261], a stimulation of mitochondrial content in liver [260] and skeletal muscle [253] and an increase in scAT and skeletal muscle oxidative capacity (ex-vivo) was observed after Res supplementation, thus displaying its caloric restriction mimicking effects under conditions of energy excess. In-vitro AT models have shown that resveratrol's anti-inflammatory effects can be attributed to a suppression of nuclear factor (NF)-kappaB activity and a decreased release and expression of proinflammatory cytokines like IL-6, IL-8, MCP-1 [262], PAI-1 [263] and TNF-alpha [264].

Compared to rodents [265], most human studies have not found a significant effect of Res on AT mass and body weight [266-269]. However, a 30-day intervention trial in obese men with 150 $\mathrm{mg} / \mathrm{d}$ Res led to a change in AT morphology presented by a reduced mean adipocyte size and gene expression changes indicative of enhanced adipogenesis and alternative lipid breakdown [270]. Nevertheless, resveratrol appears to affect whole-body metabolic profiles, substrate utilization and mitochondrial function of obese and diabetic subjects. Timmers et al. [267] reported a reduction in plasma glucose and lipid levels, inflammation markers and postprandial AT lipolysis in healthy obese men, while the oxidative capacity of skeletal muscle (ex-vivo) and the HOMA-IR index were improved after 30 days Res supplementation. These changes where reflected by a down-regulation of pathways linked to inflammation and an up-regulation of gene sets related to mitochondrial oxidative phosphorylation in muscle and may support an improved metabolic flexibility and insulin sensitivity in obese subjects. Interestingly, other groups could not find similar metabolic improvements [266, 268, 269], whereas Bhatt et al. [271] and Brasnyo et al. [272] reported beneficial effects of Res as add-on therapy or as sole supplementation in diabetic subjects, contrary to a recent study by Timmers et al. in metformin-treated T2DM subjects [273]. Hence, further intervention studies with resveratrol in well-defined subject populations are needed to elucidate if this bio-active polyphenol, with caloric-restriction like effects, could be a beneficial supplement or add-on therapy for metabolically impaired humans [265, 274]. 


\section{Polyunsaturated fatty acids}

The omega-3 (n-3) and omega-6 (n-6) fatty acids are long-chain polyunsaturated fatty acids (LCPUFAs), which are essential for humans because they cannot be synthesized de novo but are vital for normal cell and tissue metabolism. Eicosapentaenoic acid (EPA-20:5) (Table 1.3) and docosahexaenoic acid (DHA-22:6) are n-3 LC-PUFAs found in fish and algae and have attracted a lot of attention over the last 30 years. Several studies in humans and rodents have suggested potential beneficial effects of these marine PUFAs in numerous metabolic diseases with chronic inflammation including CVD, atherosclerosis, obesity and metabolic syndrome $[178,275]$.

The dietary fatty acids $\boldsymbol{E P} \boldsymbol{A}$ and DHA were also extensively studied for their effects on white AT metabolism and function. In brief, n-3 LC-PUFAs can reduce the LD formation in murine adipocytes compared to saturated FAs [276] but they also showed conflicting data concerning preadipocyte differentiation [277, 278]. Furthermore, several murine and human adipocyte assays and rodent studies, using high-fat obesogenic conditions (see [175] and references therein), demonstrated that marine EPA and DHA modulate adipocyte TAG storage and mobilization [279], by stimulating mitochondrial biogenesis, FA oxidation [280, 281] and lipolysis [276]. The beneficial effects of EPA on adipocyte glucose and lipid metabolism and on insulin sensitivity [279] in obese / diabetic rodents can partly be attributed to their PPAR and AMPK activation [282] and their modulation of cyto-/adipokine secretion [283]. The attenuated AT inflammation caused by EPA and DHA may be explained by a diminished release of pro-inflammatory chemokines and cytokines as result of reduced M1-macrophage infiltration and adipocyte hypertrophy [284]. Furthermore, EPA and DHA promote the formation of beneficial and protective n-3 PUFA derived pro-resolving lipid mediators (resolvins and protectins) and the reduction in n-6 PUFA derived pro-inflammatory lipid mediators, contributing to the resolution of acute and chronic inflammation [207, 285]. Pro-resolving mediators also exert distinct insulin sensitizing and glucose lowering activity as shown in obese-diabetic mice [286, 287]. Results in diabetic and obese subjects after the supplementation with EPA and DHA or fish oil are conflicting regarding plasma lipid and glucose levels, insulin sensitivity and adiposity (fat mass) [178, 175], although most studies indicate that an increase in n-3 LC-PUFAs intake has positive effects on lipid metabolism but not on whole-body (HOMA-IR) or peripheral (muscle) insulin sensitivity [288-291]. However, there is increasing evidence from human intervention studies, which suggest that marine n-3 LC-PUFA supplementation affects inflammation by lowering plasma markers (CRP, IL-6 and TNF-alpha) of chronic inflammatory diseases [292] and the expression of inflammatory genes in AT [293]. Together, these data indicate that n-3 LC-PUFAs can modulate AT function and thus are valuable 
dietary components for a favorable influence on systemic insulin sensitivity and chronic inflammatory diseases.

\section{Bio-actives combinations}

Combining different nutritional bio-actives with partly distinct mechanisms of action may lead to additional and/or even synergistic effects on AT biology and functionality compared with single supplementation, which may be a promising strategy to prevent or treat metabolic diseases. So far, these combined treatments have been conducted mostly in murine in-vitro AT models or in animal models of obesity, but human in-vitro and intervention studies are scarce. The combination of polyphenols in murine and human adipocytes showed additive or synergistic inhibitory effects on the lipid accumulation [294, 295]. Such a synergistic reduction in accumulated lipids could also be demonstrated in rodent white AT, when treated with a combination of Res and quercetin [296]. An acute intake (3 days) of a polyphenol combination (EGCG, Res, soy isoflavone) increased resting and 24-h EE and fasting plasma glycerol and leptin concentrations in overweight subjects [297]. These results suggest that supplementing combinations of polyphenols might be beneficial for weight maintenance and metabolic health. However, whether these beneficial acute effects could be retained on the long-term still need to be investigated in a well-controlled human setting.

\section{Outline of the thesis}

The research presented in this thesis investigated the effects of different nutritional bio-actives (trans-resveratrol, EGCG, lycopene and EPA) on AT biology and metabolic profiles in humans, with focus on adipocyte functional parameters.

The initial step was to develop a high content, cell-based assay using automated fluorescent microscopy to determine the accumulation of cytosolic LDs in differentiated C3H10 T1/2 adipocytes as described in Chapter 2. Additionally, we measured the effects of several dietary constituents on LD accumulation. In Chapter 3 we tested individual and combinations of bioactive dietary constituents including Lyc, EPA, and Res for their effects on adipocyte function of in-vitro differentiating human adipocytes (14 days), applying the fluorescence assay established and validated for LD quantification in Chapter 2. Furthermore, the influence of these bio-actives on adipogenic, lipogenic and lipolytic gene expression and cyto-/adipokine secretion was investigated. Based on the proof-of-concept results in Chapter 3 we studied in Chapter 4, whether the combination of Lyc/Res likewise ameliorates adipocyte function of in-vitro differentiating (14 days) hMADS cells that were isolated from subcutaneous white AT of age-matched lean and obese donors. For Chapter 5 and Chapter 6 a 12-week randomized, double-blind, placebo-controlled 
clinical trial was conducted to investigate whether a combined EGCG and Res supplementation has effects on metabolic profiles, skeletal muscle oxidative capacity, lipid oxidation, lipolysis and peripheral, hepatic and AT insulin sensitivity in overweight and obese, non-diabetic subjects. Therefore, a hyperinsulinemic-euglycemic clamp, indirect calorimetry and microdialysis in AT and skeletal muscle were performed before and after intervention in overweight-obese subjects (Chapter 5). Underlying molecular mechanisms were investigated in skeletal muscle biopsies using gene microarrays. Chapter 6 describes the effects of the 12-week supplementation with EGCG and Res on adipocyte size, mitochondrial protein content and transcriptional profile in abdominal subcutaneous AT compared to placebo treatment. Finally, in Chapter 7 the main findings are discussed in a broader frame and perspectives and implications for future research are indicated. 


\section{References}

1. Bastard, J.-P. and B. Fève, Physiology and Physiopathology of adipose tissue. 2012: Springer Science \& Business Media.

2. Hepler, C. and R.K. Gupta, The expanding problem of adipose depot remodeling and postnatal adipocyte progenitor recruitment. Molecular and Cellular Endocrinology, 2016.

3. Ng, M., et al., Global, regional, and national prevalence of overweight and obesity in children and adults during 1980-2013: a systematic analysis for the Global Burden of Disease Study 2013. Lancet, 2014. 384(9945): p. 766-81.

4. Vandevijvere, S., et al., Increased food energy supply as a major driver of the obesity epidemic: a global analysis. Bull World Health Organ, 2015. 93(7): p. 446-56.

5. $\quad$ Soechtig, S., Fed Up. 2014, RADiUS-TWC p. $92 \mathrm{~min}$.

6. Strasser, B., Physical activity in obesity and metabolic syndrome. Ann N Y Acad Sci, 2013. 1281: p. 141-59.

7. WHO. Obesity and overweight - Fact sheet No. 311. 2017 October 2017 [cited 2017 February 07].

8. Guilherme, A., et al., Adipocyte dysfunctions linking obesity to insulin resistance and type 2 diabetes. Nat Rev Mol Cell Biol, 2008. 9(5): p. 367-377.

9. Formiguera, X. and A. Canton, Obesity: epidemiology and clinical aspects. Best Pract Res Clin Gastroenterol, 2004. 18(6): p. 1125-1146.

10. Mandal, S. and N. Hart, Respiratory complications of obesity. Clinical Medicine, 2012. 12(1): p. 75-78.

11. Starley, B.Q., C.J. Calcagno, and S.A. Harrison, Nonalcoholic fatty liver disease and hepatocellular carcinoma: a weighty connection. Hepatology, 2010. 51(5): p. 1820-32.

12. Arnold, M., et al., Obesity and cancer: An update of the global impact. Cancer Epidemiology, 2016. 41: p. 8-15.

13. Heyward, F.D., et al., Obesity Weighs down Memory through a Mechanism Involving the Neuroepigenetic Dysregulation of Sirt1. The Journal of Neuroscience, 2016. 36(4): p. 1324-1335.

14. Klimcakova, E., et al., Adipokines and dietary interventions in human obesity. Obesity reviews, 2010(6).

15. Tang, W., et al., Thiazolidinediones regulate adipose lineage dynamics. Cell Metab, 2011. 14(1): p. $116-22$.

16. Hsu, C.L. and G.C. Yen, Phenolic compounds: evidence for inhibitory effects against obesity and their underlying molecular signaling mechanisms. Molecular nutrition \& food research, 2008. 52(1): p. 53-61.

17. Calder, P.C., et al., Dietary factors and low-grade inflammation in relation to overweight and obesity. Br J Nutr, 2011. 106 Suppl 3: p. S5-78.

18. Rosen, E.D. and B.M. Spiegelman, What we talk about when we talk about fat. Cell, 2014. 156(12): p. 20-44.

19. Wu, J., et al., Beige adipocytes are a distinct type of thermogenic fat cell in mouse and human. Cell, 2012. 150(2): p. 366-76.

20. Nedergaard, J., T. Bengtsson, and B. Cannon, Unexpected evidence for active brown adipose tissue in adult humans. American Journal of Physiology - Endocrinology And Metabolism, 2007. 293(2): p. E444.

21. van Marken Lichtenbelt, W.D., et al., Cold-activated brown adipose tissue in healthy men. N Engl J Med, 2009. 360(15): p. 1500-8.

22. Virtanen, K.A., et al., Functional brown adipose tissue in healthy adults. N Engl J Med, 2009. 360(15): p. 1518-25.

23. Perrini, S., et al., Differences in gene expression and cytokine release profiles highlight the heterogeneity of distinct subsets of adipose tissue-derived stem cells in the subcutaneous and visceral adipose tissue in humans. PLoS One, 2013. 8(3): p. e57892.

24. Tchkonia, T., et al., Mechanisms and metabolic implications of regional differences among fat depots. Cell Metab, 2013. 17(5): p. 644-56.

25. Tchoukalova, Y.D., et al., Sex- and depot-dependent differences in adipogenesis in normal-weight humans. Obesity (Silver Spring), 2010. 18(10): p. 1875-80. 
26. Shepherd, P.R. and B.B. Kahn, Glucose transporters and insulin action--implications for insulin resistance and diabetes mellitus. N Engl J Med, 1999. 341(4): p. 248-57.

27. Rosen, E.D. and B.M. Spiegelman, Adipocytes as regulators of energy balance and glucose homeostasis. Nature, 2006. 444(7121): p. 847-53.

28. Abel, E.D., et al., Adipose-selective targeting of the GLUT4 gene impairs insulin action in muscle and liver. Nature, 2001. 409(6821): p. 729.

29. Carvalho, E., et al., Adipose-specific overexpression of GLUT4 reverses insulin resistance and diabetes in mice lacking GLUT4 selectively in muscle. The American Journal of Physiology, 2005(4): p. 551.

30. Garvey, W.T., et al., Pretranslational suppression of a glucose transporter protein causes insulin resistance in adipocytes from patients with non-insulin-dependent diabetes mellitus and obesity. J Clin Invest, 1991. 87(3): p. 1072-81.

31. Ducluzeau, P.H., et al., Regulation by insulin of gene expression in human skeletal muscle and adipose tissue. Evidence for specific defects in type 2 diabetes. Diabetes, 2001. 50(5): p. 1134-42.

32. Blüher, M., Adipose tissue dysfunction contributes to obesity related metabolic diseases. Best Practice \& Research Clinical Endocrinology \& Metabolism, 2013. 27(2): p. 163-177.

33. Lanthier, N. and I.A. Leclercq, Adipose tissues as endocrine target organs. Best Practice \& Research Clinical Gastroenterology, 2014. 28(4): p. 545-558.

34. Shimizu, I. and K. Walsh, The Whitening of Brown Fat and Its Implications for Weight Management in Obesity. Curr Obes Rep, 2015. 4(2): p. 224-9.

35. Ravussin, E., et al., Determinants of 24-hour energy expenditure in man. Methods and results using a respiratory chamber. J Clin Invest, 1986. 78(6): p. 1568-78.

36. Esteve Rafols, M., Adipose tissue: cell heterogeneity and functional diversity. Endocrinol Nutr, 2014. 61(2): p. 100-12.

37. Tauchi-Sato, K., et al., The surface of lipid droplets is a phospholipid monolayer with a unique Fatty Acid composition. J Biol Chem, 2002. 277(46): p. 44507-12.

38. Sztalryd, C. and A.R. Kimmel, Perilipins: Lipid droplet coat proteins adapted for tissue-specific energy storage and utilization, and lipid cytoprotection. Biochimie, 2014. 96: p. 96-101.

39. Murphy, S., S. Martin, and R.G. Parton, Lipid droplet-organelle interactions; sharing the fats. Biochim Biophys Acta, 2009. 1791(6): p. 441-7.

40. Rambold, Angelika S., S. Cohen, and J. Lippincott-Schwartz, Fatty Acid Trafficking in Starved Cells: Regulation by Lipid Droplet Lipolysis, Autophagy, and Mitochondrial Fusion Dynamics. Developmental Cell, 2015. 32(6): p. 678-692.

41. Hashemi, H.F. and J.M. Goodman, The life cycle of lipid droplets. Current Opinion in Cell Biology, 2015. 33: p. 119-124.

42. Gong, J., et al., Fsp27 promotes lipid droplet growth by lipid exchange and transfer at lipid droplet contact sites. The Journal of Cell Biology, 2011. 195(6): p. 953-963.

43. Sun, Z., et al., Perilipin1 promotes unilocular lipid droplet formation through the activation of Fsp27 in adipocytes. Nat Commun, 2013. 4: p. 1594.

44. Lass, A., et al., Lipolysis - a highly regulated multi-enzyme complex mediates the catabolism of cellular fat stores. Prog Lipid Res, 2011. 50(1): p. 14-27.

45. Spalding, K.L., et al., Dynamics of fat cell turnover in humans. Nature, 2008. 453(7196): p. $783-$ 787.

46. Arner, P., et al., Dynamics of human adipose lipid turnover in health and metabolic disease. Nature, 2011. 478(7367): p. 110-3.

47. Spalding, K.L., et al., Impact of fat mass and distribution on lipid turnover in human adipose tissue. Nat Commun, 2017. 8: p. 15253.

48. Frayn, K.N., Adipose tissue as a buffer for daily lipid flux. Diabetologia, 2002. 45(9): p. 1201-10.

49. Oberbauer, E., et al., Enzymatic and non-enzymatic isolation systems for adipose tissue-derived cells: current state of the art. Cell Regeneration, 2015. 4(1): p. 7.

50. Tontonoz, P., E. Hu, and B.M. Spiegelman, Stimulation of adipogenesis in fibroblasts by PPAR gamma 2, a lipid-activated transcription factor. Cell, 1994. 79(7): p. 1147-1156.

51. Freytag, S.O., D.L. Paielli, and J.D. Gilbert, Ectopic expression of the CCAAT/enhancer-binding protein alpha promotes the adipogenic program in a variety of mouse fibroblastic cells. Genes Dev, 1994. 8(14): p. 1654-1663. 
52. Auwerx, J., P. Leroy, and K. Schoonjans, Lipoprotein lipase: recent contributions from molecular biology. Critical reviews in clinical laboratory sciences, 1992. 29(3-4): p. 243-268.

53. Matarese, V. and D.A. Bernlohr, Purification of murine adipocyte lipid-binding protein. Characterization as a fatty acid- and retinoic acid-binding protein. J Biol Chem, 1988. 263(28): p. 14544-14551.

54. Kim, J.B., et al., Nutritional and insulin regulation of fatty acid synthetase and leptin gene expression through ADD1/SREBP1. The Journal of clinical investigation, 1998. 101(1): p. 1-9.

55. Sztalryd, C., M.C. Komaromy, and F.B. Kraemer, Overexpression of hormone-sensitive lipase prevents triglyceride accumulation in adipocytes. The Journal of clinical investigation, 1995. 95(6): p. 2652-2661.

56. James, D.E., et al., Insulin-regulatable tissues express a unique insulin-sensitive glucose transport protein. Nature, 1988. 333(6169): p. 183-185.

57. Lowe, C.E., S. O'Rahilly, and J.J. Rochford, Adipogenesis at a glance. Journal of Cell Science, 2011. 124(16): p. 2681-2686.

58. Eissing, L., et al., De novo lipogenesis in human fat and liver is linked to ChREBP- $\beta$ and metabolic health. Nature communications, 2013. 4: p. 1528-1528.

59. Kersten, S., Mechanisms of nutritional and hormonal regulation of lipogenesis. 2001. p. 282.

60. Guo, Z.K., et al., De novo lipogenesis in adipose tissue of lean and obese women: application of deuterated water and isotope ratio mass spectrometry. Int J Obes Relat Metab Disord, 2000. 24(7): p. 932-7.

61. Diraison, F., et al., Differences in the regulation of adipose tissue and liver lipogenesis by carbohydrates in humans. J Lipid Res, 2003. 44(4): p. 846-53.

62. Kersten, S., Physiological regulation of lipoprotein lipase. Biochim Biophys Acta, 2014. 1841(7): p. 919-33.

63. Dijk, W. and S. Kersten, Regulation of lipoprotein lipase by Angpt14. Trends Endocrinol Metab, 2014. 25(3): p. 146-55.

64. Fielding, B.A. and K.N. Frayn, Lipoprotein lipase and the disposition of dietary fatty acids. Br J Nutr, 1998. 80(6): p. 495-502.

65. Kazantzis, M. and A. Stahl, Fatty acid transport proteins, implications in physiology and disease. Biochimica Et Biophysica Acta, 2012. 1821(5): p. 852-857.

66. Hajri, T. and N.A. Abumrad, FATTY ACID TRANSPORT ACROSS MEMBRANES: Relevance to Nutrition and Metabolic Pathology. Annual Review of Nutrition, 2002. 22(1): p. 383.

67. Furuhashi, M. and G.S. Hotamisligil, Fatty acid-binding proteins: role in metabolic diseases and potential as drug targets. Nat Rev Drug Discov, 2008. 7(6): p. 489-503.

68. Ellis, J.M., et al., Acyl-coenzyme A synthetases in metabolic control. Curr Opin Lipidol, 2010. 21(3): p. 212-7.

69. Shi, Y. and D. Cheng, Beyond triglyceride synthesis: the dynamic functional roles of MGAT and DGAT enzymes in energy metabolism. American Journal of Physiology - Endocrinology And Metabolism, 2009. 297(1): p. E10.

70. Czech, M.P., et al., Insulin signalling mechanisms for triacylglycerol storage. Diabetologia, 2013. 56(5): p. 949-964.

71. Nielsen, T.S., et al., Dissecting adipose tissue lipolysis: molecular regulation and implications for metabolic disease. J Mol Endocrinol, 2014. 52(3): p. R199-222.

72. Schweiger, M., et al., Adipose triglyceride lipase and hormone-sensitive lipase are the major enzymes in adipose tissue triacylglycerol catabolism. J Biol Chem, 2006. 281(52): p. 40236-41.

73. Greenberg, A.S., et al., Perilipin, a major hormonally regulated adipocyte-specific phosphoprotein associated with the periphery of lipid storage droplets. J Biol Chem, 1991. 266(17): p. 11341-6.

74. Brasaemle, D.L., Thematic review series: adipocyte biology. The perilipin family of structural lipid droplet proteins: stabilization of lipid droplets and control of lipolysis. J Lipid Res, 2007. 48(12): p. 2547-59.

75. Grahn, T.H., et al., Fat-specific protein 27 (FSP27) interacts with adipose triglyceride lipase (ATGL) to regulate lipolysis and insulin sensitivity in human adipocytes. J Biol Chem, 2014. 289(17): p. 12029-39.

76. Lass, A., et al., Adipose triglyceride lipase-mediated lipolysis of cellular fat stores is activated by CGI-58 and defective in Chanarin-Dorfman Syndrome. Cell Metab, 2006. 3(5): p. 309-19. 
77. Zimmermann, R., et al., Fat mobilization in adipose tissue is promoted by adipose triglyceride lipase. Science, 2004. 306(5700): p. 1383-6.

78. Egan, J.J., et al., Mechanism of hormone-stimulated lipolysis in adipocytes: translocation of hormone-sensitive lipase to the lipid storage droplet. Proc Natl Acad Sci U S A, 1992. 89(18): p. 8537-41.

79. Ryden, M., et al., Comparative studies of the role of hormone-sensitive lipase and adipose triglyceride lipase in human fat cell lipolysis. Am J Physiol Endocrinol Metab, 2007. 292(6): p. E1847-55.

80. Fredrikson, G., H. Tornqvist, and P. Belfrage, Hormone-sensitive lipase and monoacylglycerol lipase are both required for complete degradation of adipocyte triacylglycerol. Biochim Biophys Acta, 1986. 876(2): p. 288-93.

81. Fruhbeck, G., et al., Regulation of adipocyte lipolysis. Nutr Res Rev, 2014. 27(1): p. 63-93.

82. Schweiger, M., et al., G0/G1 switch gene-2 regulates human adipocyte lipolysis by affecting activity and localization of adipose triglyceride lipase. Journal of Lipid Research, 2012. 53(11): p. 2307-2317.

83. Sengenes, C., et al., Natriuretic peptides: a new lipolytic pathway in human adipocytes. The FASEB Journal, 2000. 14(10): p. 1345-1351.

84. Moro, C., et al., Differential regulation of atrial natriuretic peptide- and adrenergic receptordependent lipolytic pathways in human adipose tissue. Metabolism, 2005. 54(1): p. 122-31.

85. Goossens, G.H., The role of adipose tissue dysfunction in the pathogenesis of obesity-related insulin resistance. Physiol Behav, 2008. 94(2): p. 206-18.

86. Stinkens, R., et al., Targeting fatty acid metabolism to improve glucose metabolism. Obesity reviews, 2015(9).

87. Zhang, Y., et al., Positional cloning of the mouse obese gene and its human homologue. Nature, 1994. 372(6505): p. 425-32.

88. Galic, S., J.S. Oakhill, and G.R. Steinberg, Adipose tissue as an endocrine organ. Molecular and Cellular Endocrinology, 2010. 316(2): p. 129-139.

89. Ronti, T., G. Lupattelli, and E. Mannarino, The endocrine function of adipose tissue: an update. Clin Endocrinol (Oxf), 2006. 64(4): p. 355-65.

90. Antuna-Puente, B., et al., Adipokines: the missing link between insulin resistance and obesity. Diabetes Metab, 2008. 34(1): p. 2-11.

91. Nakamura, K., J.J. Fuster, and K. Walsh, Adipokines: a link between obesity and cardiovascular disease. J Cardiol, 2014. 63(4): p. 250-9.

92. $\mathrm{Xu}, \mathrm{H}$., et al., Chronic inflammation in fat plays a crucial role in the development of obesity-related insulin resistance. J Clin Invest, 2003. 112(12): p. 1821-30.

93. Wang, B., J.R. Jenkins, and P. Trayhurn, Expression and secretion of inflammation-related adipokines by human adipocytes differentiated in culture: integrated response to TNF-alpha. Am J Physiol Endocrinol Metab, 2005. 288(4): p. E731-40.

94. Skurk, T., et al., Relationship between adipocyte size and adipokine expression and secretion. J Clin Endocrinol Metab, 2007. 92(3): p. 1023-33.

95. Bluher, M., Adipose tissue inflammation: a cause or consequence of obesity-related insulin resistance? Clin Sci (Lond), 2016. 130(18): p. 1603-14.

96. Chawla, A., K.D. Nguyen, and Y.P. Goh, Macrophage-mediated inflammation in metabolic disease. Nat Rev Immunol, 2011. 11(11): p. 738-49.

97. Huh, J.Y., et al., Crosstalk between Adipocytes and Immune Cells in Adipose Tissue Inflammation and Metabolic Dysregulation in Obesity. Molecules and Cells, 2014. 37(5): p. 365-371.

98. Weisberg, S.P., et al., Obesity is associated with macrophage accumulation in adipose tissue. J Clin Invest, 2003. 112(12): p. 1796-808.

99. Harman-Boehm, I., et al., Macrophage infiltration into omental versus subcutaneous fat across different populations: effect of regional adiposity and the comorbidities of obesity. J Clin Endocrinol Metab, 2007. 92(6): p. 2240-7.

100. Cancello, R., et al., Reduction of macrophage infiltration and chemoattractant gene expression changes in white adipose tissue of morbidly obese subjects after surgery-induced weight loss. Diabetes, 2005. 54(8): p. 2277-86. 
101. Yamauchi, T., et al., The fat-derived hormone adiponectin reverses insulin resistance associated with both lipoatrophy and obesity. Nat Med, 2001. 7(8): p. 941-6.

102. Wang, Y., et al., Post-translational modifications of adiponectin: mechanisms and functional implications. Biochem J, 2008. 409(3): p. 623-33.

103. Yamauchi, T., et al., Cloning of adiponectin receptors that mediate antidiabetic metabolic effects. Nature, 2003. 423(6941): p. 762-9.

104. Lo, J.C., et al., Adipsin is an adipokine that improves beta cell function in diabetes. Cell, 2014. 158(1): p. 41-53.

105. Derosa, G., et al., Adipocytokine levels in obese and non-obese subjects: an observational study. Inflammation, 2013. 36(4): p. 914-20.

106. McGee, K.C., et al., Visfatin Is Regulated by Rosiglitazone in Type 2 Diabetes Mellitus and Influenced by NFKB and JNK in Human Abdominal Subcutaneous Adipocytes. PLoS ONE, 2011. 6(6): p. e20287.

107. Gautron, L. and J.K. Elmquist, Sixteen years and counting: an update on leptin in energy balance. J Clin Invest, 2011. 121(6): p. 2087-93.

108. Sainz, N., et al., Leptin resistance and diet-induced obesity: central and peripheral actions of leptin. Metabolism, 2015. 64(1): p. 35-46.

109. Turer, A.T., et al., Adipose tissue mass and location affect circulating adiponectin levels. Diabetologia, 2011. 54(10): p. 2515-24.

110. Castan-Laurell, I., et al., Apelin, diabetes, and obesity. Endocrine, 2011. 40(1): p. 1-9.

111. Sun, K., C.M. Kusminski, and P.E. Scherer, Adipose tissue remodeling and obesity. J Clin Invest, 2011. 121(6): p. 2094-101.

112. Alligier, M., et al., Subcutaneous adipose tissue remodeling during the initial phase of weight gain induced by overfeeding in humans. J Clin Endocrinol Metab, 2012. 97(2): p. E183-92.

113. Arner, E., et al., Adipocyte turnover: relevance to human adipose tissue morphology. Diabetes, 2010. 59(1): p. 105-9.

114. Tan, C.Y. and A. Vidal-Puig, Adipose tissue expandability: the metabolic problems of obesity may arise from the inability to become more obese. Biochem Soc Trans, 2008. 36(Pt 5): p. 935-40.

115. Krotkiewski, M., et al., Impact of obesity on metabolism in men and women. Importance of regional adipose tissue distribution. J Clin Invest, 1983. 72(3): p. 1150-62.

116. Tchoukalova, Y.D., et al., Regional differences in cellular mechanisms of adipose tissue gain with overfeeding. Proc Natl Acad Sci U S A, 2010. 107(42): p. 18226-31.

117. Manolopoulos, K.N., F. Karpe, and K.N. Frayn, Gluteofemoral body fat as a determinant of metabolic health. Int J Obes, 2010. 34(6): p. 949-959.

118. Karpe, F. and K.E. Pinnick, Biology of upper-body and lower-body adipose tissue--link to wholebody phenotypes. Nat Rev Endocrinol, 2015. 11(2): p. 90-100.

119. Trayhurn, P., Hypoxia and adipose tissue function and dysfunction in obesity. Physiol Rev, 2013. 93(1): p. 1-21.

120. Goossens, G.H. and E.E. Blaak, Adipose tissue dysfunction and impaired metabolic health in human obesity: a matter of oxygen? Front Endocrinol (Lausanne), 2015. 6: p. 55.

121. Kim, J.I., et al., Lipid-overloaded enlarged adipocytes provoke insulin resistance independent of inflammation. Mol Cell Biol, 2015. 35(10): p. 1686-99.

122. Michaud, A., et al., Abdominal subcutaneous and omental adipocyte morphology and its relation to gene expression, lipolysis and adipocytokine levels in women. Metabolism, 2014. 63(3): p. 37281.

123. Sun, K., et al., Fibrosis and adipose tissue dysfunction. Cell Metab, 2013. 18(4): p. 470-7.

124. Cinti, S., et al., Adipocyte death defines macrophage localization and function in adipose tissue of obese mice and humans. J Lipid Res, 2005. 46(11): p. 2347-55.

125. Gustafson, B., et al., Inflammation and impaired adipogenesis in hypertrophic obesity in man. Am J Physiol Endocrinol Metab, 2009. 297(5): p. E999-e1003.

126. Kloting, N., et al., Insulin-sensitive obesity. Am J Physiol Endocrinol Metab, 2010. 299(3): p. E506-15.

127. Ryden, M., et al., Adipocyte triglyceride turnover and lipolysis in lean and overweight subjects. J Lipid Res, 2013. 54(10): p. 2909-13. 
128. Hammarstedt, A., T.E. Graham, and B.B. Kahn, Adipose tissue dysregulation and reduced insulin sensitivity in non-obese individuals with enlarged abdominal adipose cells. Diabetology \& Metabolic Syndrome, 2012. 4: p. 42-42.

129. Arner, P. and D. Langin, Lipolysis in lipid turnover, cancer cachexia, and obesity-induced insulin resistance. Trends Endocrinol Metab, 2014. 25(5): p. 255-62.

130. Semenkovich, C.F., et al., Insulin regulation of lipoprotein lipase activity in 3T3-L1 adipocytes is mediated at posttranscriptional and posttranslational levels. J Biol Chem, 1989. 264(15): p. 90308.

131. Bickerton, A.S., et al., Adipose tissue fatty acid metabolism in insulin-resistant men. Diabetologia, 2008. 51(8): p. 1466-74.

132. Jocken, J.W., et al., Adipose triglyceride lipase and hormone-sensitive lipase protein expression is decreased in the obese insulin-resistant state. J Clin Endocrinol Metab, 2007. 92(6): p. 2292-9.

133. Jocken, J.W., et al., Insulin-mediated suppression of lipolysis in adipose tissue and skeletal muscle of obese type 2 diabetic men and men with normal glucose tolerance. Diabetologia, 2013. 56(10): p. 2255-65.

134. Jocken, J.W. and E.E. Blaak, Catecholamine-induced lipolysis in adipose tissue and skeletal muscle in obesity. Physiol Behav, 2008. 94(2): p. 219-30.

135. Verboven, K., et al., Attenuated atrial natriuretic peptide-mediated lipolysis in subcutaneous adipocytes of obese type 2 diabetic men. Clin Sci (Lond), 2016. 130(13): p. 1105-14.

136. Kovsan, J., et al., Altered autophagy in human adipose tissues in obesity. J Clin Endocrinol Metab, 2011. 96(2): p. E268-77.

137. Haim, Y., et al., Elevated autophagy gene expression in adipose tissue of obese humans: A potential non-cell-cycle-dependent function of E2F1. Autophagy, 2015. 11(11): p. 2074-2088.

138. Soussi, H., et al., DAPK2 Downregulation Associates With Attenuated Adipocyte Autophagic Clearance in Human Obesity. Diabetes, 2015. 64(10): p. 3452-63.

139. Farese, R.V., Jr. and T.C. Walther, Lipid droplets finally get a little R-E-S-P-E-C-T. Cell, 2009. 139(5): p. 855-60.

140. Muoio, D.M., Metabolic inflexibility: when mitochondrial indecision leads to metabolic gridlock. Cell, 2014. 159(6): p. 1253-62.

141. Kusminski, C.M. and P.E. Scherer, Mitochondrial dysfunction in white adipose tissue. Trends Endocrinol Metab, 2012. 23(9): p. 435-43.

142. Yin, X., et al., Adipocyte Mitochondrial Function Is Reduced in Human Obesity Independent of Fat Cell Size. The Journal of Clinical Endocrinology and Metabolism, 2014. 99(2): p. E209-E216.

143. Jocken, J.W., et al., Contribution of lipase deficiency to mitochondrial dysfunction and insulin resistance in hMADS adipocytes. Int J Obes (Lond), 2016. 40(3): p. 507-13.

144. Haemmerle, G., et al., ATGL-mediated fat catabolism regulates cardiac mitochondrial function via PPAR-alpha and PGC-1. Nat Med, 2011. 17(9): p. 1076-85.

145. Engin, A., The Pathogenesis of Obesity-Associated Adipose Tissue Inflammation. Adv Exp Med Biol, 2017. 960: p. 221-245.

146. Kim, C.S., et al., Circulating levels of MCP-1 and IL-8 are elevated in human obese subjects and associated with obesity-related parameters. Int J Obes (Lond), 2006. 30(9): p. 1347-55.

147. Mraz, M. and M. Haluzik, The role of adipose tissue immune cells in obesity and low-grade inflammation. J Endocrinol, 2014. 222(3): p. R113-27.

148. Degawa-Yamauchi, M., et al., Regulation of adiponectin expression in human adipocytes: effects of adiposity, glucocorticoids, and tumor necrosis factor alpha. Obes Res, 2005. 13(4): p. 662-9.

149. Lacasa, D., et al., Macrophage-secreted factors impair human adipogenesis: involvement of proinflammatory state in preadipocytes. Endocrinology, 2007. 148(2): p. 868-77.

150. Sun, S., et al., Mechanisms of inflammatory responses in obese adipose tissue. Annu Rev Nutr, 2012. 32: p. 261-86.

151. Chehimi, M., H. Vidal, and A. Eljaafari, Pathogenic Role of IL-17-Producing Immune Cells in Obesity, and Related Inflammatory Diseases. J Clin Med, 2017. 6(7).

152. Wernstedt Asterholm, I., et al., Adipocyte inflammation is essential for healthy adipose tissue expansion and remodeling. Cell Metab, 2014. 20(1): p. 103-18.

153. Montague, C.T. and S. O'Rahilly, The perils of portliness: causes and consequences of visceral adiposity. Diabetes, 2000. 49(6): p. 883-8. 
154. Heilbronn, L., S.R. Smith, and E. Ravussin, Failure of fat cell proliferation, mitochondrial function and fat oxidation results in ectopic fat storage, insulin resistance and type II diabetes mellitus. Int J Obes Relat Metab Disord, 2004. 28 Suppl 4: p. S12-21.

155. Jensen, M.D., Is visceral fat involved in the pathogenesis of the metabolic syndrome? Human model. Obesity (Silver Spring), 2006. 14 Suppl 1: p. 20s-24s.

156. Nielsen, S. and F. Karpe, Determinants of VLDL-triglycerides production. Curr Opin Lipidol, 2012. 23(4): p. 321-6.

157. van Hees, A.M., et al., Skeletal muscle fatty acid handling in insulin resistant men. Obesity (Silver Spring), 2011. 19(7): p. 1350-9.

158. Shulman, G.I., Ectopic Fat in Insulin Resistance, Dyslipidemia, and Cardiometabolic Disease. N Engl J Med, 2014. 371(12): p. 1131-1141.

159. van der Kolk, B.W., et al., Altered skeletal muscle fatty acid handling is associated with the degree of insulin resistance in overweight and obese humans. Diabetologia, 2016. 59(12): p. 2686-2696.

160. Bremer, A.A. and I. Jialal, Adipose tissue dysfunction in nascent metabolic syndrome. J Obes, 2013. 2013: p. 393192.

161. Kadowaki, T., et al., Adiponectin and adiponectin receptors in insulin resistance, diabetes, and the metabolic syndrome. J Clin Invest, 2006. 116(7): p. 1784-92.

162. Mitrou, P., et al., Rates of lipid fluxes in adipose tissue in vivo after a mixed meal in morbid obesity. Int J Obes (Lond), 2010. 34(4): p. 770-4.

163. Yang, Q., et al., Serum retinol binding protein 4 contributes to insulin resistance in obesity and type 2 diabetes. Nature, 2005. 436(7049): p. 356-62.

164. Bluher, M., The distinction of metabolically 'healthy' from 'unhealthy' obese individuals. Curr Opin Lipidol, 2010. 21(1): p. 38-43.

165. Green, H. and M. Meuth, An established pre-adipose cell line and its differentiation in culture. Cell, 1974. 3(2): p. 127-133.

166. Reznikoff, C.A., D.W. Brankow, and C. Heidelberger, Establishment and characterization of a cloned line of $\mathrm{C} 3 \mathrm{H}$ mouse embryo cells sensitive to postconfluence inhibition of division. Cancer Res, 1973. 33(12): p. 3231-3238.

167. Armani, A., et al., Cellular models for understanding adipogenesis, adipose dysfunction, and obesity. J Cell Biochem, 2010. 110(3): p. 564-72.

168. Rodriguez, A.M., et al., The human adipose tissue is a source of multipotent stem cells. Biochimie, 2005. 87(1): p. 125-8.

169. Ruiz-Ojeda, F.J., et al., Cell Models and Their Application for Studying Adipogenic Differentiation in Relation to Obesity: A Review. International Journal of Molecular Sciences, 2016. 17(7): p. 1040.

170. Wabitsch, M., et al., Characterization of a human preadipocyte cell strain with high capacity for adipose differentiation. Int J Obes Relat Metab Disord, 2001. 25(1): p. 8-15.

171. Todorcevic, M., et al., A cellular model for the investigation of depot specific human adipocyte biology. Adipocyte, 2017. 6(1): p. 40-55.

172. Keuper, M., et al., THP-1 Macrophages and SGBS Adipocytes - A New Human in vitro Model System of Inflamed Adipose Tissue. Frontiers in Endocrinology, 2011. 2: p. 89.

173. Aubin, K., et al., Characterization of $<$ italic $>$ In Vitro $<$ italic $>$ Engineered Human Adipose Tissues: Relevant Adipokine Secretion and Impact of TNF- $\alpha$. PLoS ONE, 2015. 10(9): p. e0137612.

174. Gonçalves, C.G., M.J. Glade, and M.M. Meguid, Review: Metabolically healthy obese individuals: Key protective factors. Nutrition, 2016. 32: p. 14-20.

175. Todorčević, M. and L. Hodson, The Effect of Marine Derived n-3 Fatty Acids on Adipose Tissue Metabolism and Function. Journal of Clinical Medicine, 2016. 5(1): p. 3.

176. Luisa Bonet, M., et al., Carotenoids and their conversion products in the control of adipocyte function, adiposity and obesity. Archives of Biochemistry and Biophysics, 2015. 572: p. 112-125.

177. Wang, S., et al., Novel insights of dietary polyphenols and obesity. J Nutr Biochem, 2014. 25(1): p. 1-18.

178. Lorente-Cebrian, S., et al., Role of omega-3 fatty acids in obesity, metabolic syndrome, and cardiovascular diseases: a review of the evidence. J Physiol Biochem, 2013. 69(3): p. 633-51.

179. Thompson, D., et al., Physical activity and exercise in the regulation of human adipose tissue physiology. Physiol Rev, 2012. 92(1): p. 157-91. 
180. Clarke, C.J. and J.N. Haselden, Metabolic profiling as a tool for understanding mechanisms of toxicity. Toxicol Pathol, 2008. 36(1): p. 140-7.

181. Jomova, K. and M. Valko, Health protective effects of carotenoids and their interactions with other biological antioxidants. Eur J Med Chem, 2013. 70: p. 102-10.

182. Linus Pauling Institute (Jane Higdon, P.D. MIC - Phytochemicals $>>$ Carotenoids. 2016 Updated in July 2016 [cited 2017 07.02.2017].

183. Bonet, M.L., et al., Carotenoids in Adipose Tissue Biology and Obesity. Subcell Biochem, 2016. 79: p. 377-414.

184. Medicine, I.o., Dietary Reference Intakes for Vitamin C, Vitamin E, Selenium, and Carotenoids. 2000, Washington, DC: The National Academies Press. 529.

185. Rao, A.V. and L.G. Rao, Carotenoids and human health. Pharmacological Research, 2007. 55(3): p. 207-216.

186. Britton, G., S. Liaaen-Jensen, and H. Pfander, Carotenoids handbook. 2004, Basel: Birkhal0308user.

187. Grune, T., et al., Beta-carotene is an important vitamin A source for humans. J Nutr, 2010. 140(12): p. $2268 \mathrm{~s}-2285 \mathrm{~s}$.

188. Gouranton, E., et al., Purified low-density lipoprotein and bovine serum albumin efficiency to internalise lycopene into adipocytes. Food Chem Toxicol, 2008. 46(12): p. 3832-6.

189. Kaulmann, A. and T. Bohn, Carotenoids, inflammation, and oxidative stress--implications of cellular signaling pathways and relation to chronic disease prevention. Nutr Res, 2014. 34(11): p. 907-29.

190. Östh, M., et al., The Concentration of $\beta$-Carotene in Human Adipocytes, but Not the Whole-Body Adipocyte Stores, Is Reduced in Obesity. PLoS ONE, 2014. 9(1): p. e85610.

191. Canas, J.A., et al., Insulin resistance and adiposity in relation to serum beta-carotene levels. J Pediatr, 2012. 161(1): p. 58-64.e1-2.

192. Maiani, G., et al., Carotenoids: actual knowledge on food sources, intakes, stability and bioavailability and their protective role in humans. Mol Nutr Food Res, 2009. 53 Suppl 2: p. S194218.

193. Stahl, W., et al., cis-trans isomers of lycopene and beta-carotene in human serum and tissues. Arch Biochem Biophys, 1992. 294(1): p. 173-7.

194. Wang, X.-D., Lycopene metabolism and its biological significance. The American Journal of Clinical Nutrition, 2012. 96(5): p. 1214S-1222S.

195. Thies, F., et al., Cardiovascular benefits of lycopene: fantasy or reality? Proc Nutr Soc, 2017. 76(2): p. 122-129.

196. Devaraj, S., et al., A dose-response study on the effects of purified lycopene supplementation on biomarkers of oxidative stress. J Am Coll Nutr, 2008. 27(2): p. 267-73.

197. Ghavipour, M., et al., Tomato juice consumption reduces systemic inflammation in overweight and obese females. Br J Nutr, 2013. 109(11): p. 2031-5.

198. Ghavipour, M., G. Sotoudeh, and M. Ghorbani, Tomato juice consumption improves blood antioxidative biomarkers in overweight and obese females. Clin Nutr, 2015. 34(5): p. 805-9.

199. Roohbakhsh, A., G. Karimi, and M. Iranshahi, Carotenoids in the treatment of diabetes mellitus and its complications: A mechanistic review. Biomed Pharmacother, 2017. 91: p. 31-42.

200. Gouranton, E., et al., Apo-10'-lycopenoic acid impacts adipose tissue biology via the retinoic acid receptors. Biochim Biophys Acta, 2011. 1811(12): p. 1105-14.

201. Gouranton, E., et al., Lycopene inhibits proinflammatory cytokine and chemokine expression in adipose tissue. J Nutr Biochem, 2011. 22(7): p. 642-8.

202. Luvizotto, R.A., et al., Lycopene-rich tomato oleoresin modulates plasma adiponectin concentration and mRNA levels of adiponectin, SIRT1, and FoxO1 in adipose tissue of obese rats. Hum Exp Toxicol, 2015. 34(6): p. 612-9.

203. Luvizotto, R.d.A.M., et al., Lycopene supplementation modulates plasma concentrations and epididymal adipose tissue mRNA of leptin, resistin and IL-6 in diet-induced obese rats. British Journal of Nutrition, 2013. 110(10): p. 1803-1809.

204. Fenni, S., et al., Lycopene and tomato powder supplementation similarly inhibit high-fat diet induced obesity, inflammatory response, and associated metabolic disorders. Mol Nutr Food Res, 2017. 
205. Aguirre, L., et al., Resveratrol: Anti-Obesity Mechanisms of Action. Molecules, 2014. 19(11): p. 18632

206. Wolfram, S., Y. Wang, and F. Thielecke, Anti-obesity effects of green tea: from bedside to bench. Molecular nutrition \& food research, 2006. 50(2): p. 176-187.

207. Martínez-Fernández, L., et al., Omega-3 fatty acids and adipose tissue function in obesity and metabolic syndrome. Prostaglandins \& Other Lipid Mediators, 2015. 121, Part A: p. 24-41.

208. Li, Y.F., et al., Tomato juice supplementation in young women reduces inflammatory adipokine levels independently of body fat reduction. Nutrition, 2015. 31(5): p. 691-6.

209. McEneny, J., et al., Lycopene intervention reduces inflammation and improves HDL functionality in moderately overweight middle-aged individuals. J Nutr Biochem, 2013. 24(1): p. 163-8.

210. Tsitsimpikou, C., et al., Dietary supplementation with tomato-juice in patients with metabolic syndrome: A suggestion to alleviate detrimental clinical factors. Food and Chemical Toxicology, 2014. 74: p. 9-13.

211. Carpene, C., et al., Novel strategies for preventing diabetes and obesity complications with natural polyphenols. Curr Med Chem, 2015. 22(1): p. 150-64.

212. Duda-Chodak, A., The inhibitory effect of polyphenols on human gut microbiota. J Physiol Pharmacol, 2012. 63(5): p. 497-503.

213. Lewandowska, H., et al., The role of natural polyphenols in cell signaling and cytoprotection against cancer development. J Nutr Biochem, 2016. 32: p. 1-19.

214. Scalbert, A. Phenol-Explorer - Database on polyphenol contents in foods (INRA). [Data Base] 2015 Version 3.6 released June 2015 07.02.2017].

215. Sasazuki, S., et al., Relation between green tea consumption and the severity of coronary atherosclerosis among Japanese men and women. Ann Epidemiol, 2000. 10(6): p. 401-8.

216. Saito, E., et al., Association of green tea consumption with mortality due to all causes and major causes of death in a Japanese population: the Japan Public Health Center-based Prospective Study (JPHC Study). Annals of Epidemiology, 2015. 25(7): p. 512-518.e3.

217. Renaud, S. and M. de Lorgeril, Wine, alcohol, platelets, and the French paradox for coronary heart disease. The Lancet, 1992. 339(8808): p. 1523-1526.

218. $\mathrm{Wu}, \mathrm{M}$., et al., Epigallocatechin-3-gallate inhibits adipogenesis through down-regulation of PPARgamma and FAS expression mediated by PI3K-AKT signaling in 3T3-L1 cells. Eur J Pharmacol, 2016. 795: p. 134-142.

219. Hung, P.F., et al., Antimitogenic effect of green tea (-)-epigallocatechin gallate on 3T3-L1 preadipocytes depends on the ERK and Cdk2 pathways. Am J Physiol Cell Physiol, 2005. 288(5): p. C1094-108.

220. Chen, S., N. Osaki, and A. Shimotoyodome, Green tea catechins enhance norepinephrine-induced lipolysis via a protein kinase A-dependent pathway in adipocytes. Biochem Biophys Res Commun, 2015. 461(1): p. 1-7.

221. Wolfram, S., et al., TEAVIGO (epigallocatechin gallate) supplementation prevents obesity in rodents by reducing adipose tissue mass. Annals of Nutrition \& Metabolism, 2005. 49(1): p. 5463.

222. Wolfram, S., et al., Epigallocatechin gallate supplementation alleviates diabetes in rodents. J Nutr, 2006. 136(10): p. 2512-8.

223. Lee, M.S., C.T. Kim, and Y. Kim, Green tea (-)-epigallocatechin-3-gallate reduces body weight with regulation of multiple genes expression in adipose tissue of diet-induced obese mice. Ann Nutr Metab, 2009. 54(2): p. 151-7.

224. Klaus, S., et al., Epigallocatechin gallate attenuates diet-induced obesity in mice by decreasing energy absorption and increasing fat oxidation. International journal of obesity (2005), 2005. 29(6): p. 615-623.

225. Yang, C.S., et al., Mechanisms of body weight reduction and metabolic syndrome alleviation by tea. Mol Nutr Food Res, 2016. 60(1): p. 160-74.

226. Chen, Y.K., et al., Effects of green tea polyphenol (-)-epigallocatechin-3-gallate on newly developed high-fat/Western-style diet-induced obesity and metabolic syndrome in mice. J Agric Food Chem, 2011. 59(21): p. 11862-71.

227. Moreno, M.F., et al., Metabolic profile response to administration of epigallocatechin-3-gallate in high-fat-fed mice. Diabetol Metab Syndr, 2014. 6(1): p. 84. 
228. Bao, S., et al., Epigallocatechin gallate improves insulin signaling by decreasing toll-like receptor 4 (TLR4) activity in adipose tissues of high-fat diet rats. Mol Nutr Food Res, 2014. 58(4): p. 67786.

229. Hsu, C.-H., et al., Effect of green tea extract on obese women: A randomized, double-blind, placebo-controlled clinical trial. Clinical Nutrition, 2008. 27(3): p. 363-370.

230. Chen, I.J., et al., Therapeutic effect of high-dose green tea extract on weight reduction: A randomized, double-blind, placebo-controlled clinical trial. Clinical Nutrition, 2016. 35(3): p. 592599.

231. Janssens, P.L., R. Hursel, and M.S. Westerterp-Plantenga, Long-term green tea extract supplementation does not affect fat absorption, resting energy expenditure, and body composition in adults. J Nutr, 2015. 145(5): p. 864-70.

232. Mielgo-Ayuso, J., et al., Effects of dietary supplementation with epigallocatechin-3-gallate on weight loss, energy homeostasis, cardiometabolic risk factors and liver function in obese women: randomised, double-blind, placebo-controlled clinical trial. Br J Nutr, 2014. 111(7): p. 1263-71.

233. Boschmann, M. and F. Thielecke, The effects of epigallocatechin-3-gallate on thermogenesis and fat oxidation in obese men: a pilot study. J Am Coll Nutr, 2007. 26(4): p. 389s-395s.

234. Thielecke, F., et al., Epigallocatechin-3-gallate and postprandial fat oxidation in overweight/obese male volunteers: a pilot study. Eur J Clin Nutr, 2010. 64(7): p. 704-13.

235. Rumpler, W., et al., Oolong tea increases metabolic rate and fat oxidation in men. J Nutr, 2001. 131(11): p. 2848-52.

236. Bogdanski, P., et al., Green tea extract reduces blood pressure, inflammatory biomarkers, and oxidative stress and improves parameters associated with insulin resistance in obese, hypertensive patients. Nutr Res, 2012. 32(6): p. 421-7.

237. Brown, A.L., et al., Effects of dietary supplementation with the green tea polyphenol epigallocatechin-3-gallate on insulin resistance and associated metabolic risk factors: randomized controlled trial. Br J Nutr, 2009. 101(6): p. 886-94.

238. Suzuki, T., et al., Beneficial Effects of Tea and the Green Tea Catechin Epigallocatechin-3-gallate on Obesity. Molecules, 2016. 21(10).

239. Legeay, S., et al., Epigallocatechin Gallate: A Review of Its Beneficial Properties to Prevent Metabolic Syndrome. Nutrients, 2015. 7(7): p. 5443-68.

240. Thielecke, F. and M. Boschmann, The potential role of green tea catechins in the prevention of the metabolic syndrome - a review. Phytochemistry, 2009. 70(1): p. 11-24.

241. Huang, J., et al., The anti-obesity effects of green tea in human intervention and basic molecular studies. Eur J Clin Nutr, 2014. 68(10): p. 1075-87.

242. Brown, A.L., et al., Health effects of green tea catechins in overweight and obese men: a randomised controlled cross-over trial. Br J Nutr, 2011. 106(12): p. 1880-9.

243. Hursel, R., et al., The Role of Catechol-O-Methyl Transferase Val(108/158)Met Polymorphism (rs4680) in the Effect of Green Tea on Resting Energy Expenditure and Fat Oxidation: A Pilot Study. PLoS ONE, 2014. 9(9): p. e106220.

244. Kapoor, M.P., et al., Physiological effects of epigallocatechin-3-gallate (EGCG) on energy expenditure for prospective fat oxidation in humans: A systematic review and meta-analysis. J Nutr Biochem, 2016. 43: p. 1-10.

245. Gambini, J., et al., Properties of Resveratrol: In Vitro and In Vivo Studies about Metabolism, Bioavailability, and Biological Effects in Animal Models and Humans. Oxid Med Cell Longev, 2015. 2015: p. 837042.

246. Timmers, S., M.K. Hesselink, and P. Schrauwen, Therapeutic potential of resveratrol in obesity and type 2 diabetes: new avenues for health benefits? Ann N Y Acad Sci, 2013. 1290: p. 83-9.

247. Raederstorff, D., I. Kunz, and J. Schwager, Resveratrol, from experimental data to nutritional evidence: the emergence of a new food ingredient. Ann N Y Acad Sci, 2013. 1290: p. 136-41.

248. Mitterberger, M.C. and W. Zwerschke, Mechanisms of resveratrol-induced inhibition of clonal expansion and terminal adipogenic differentiation in 3T3-L1 preadipocytes. J Gerontol A Biol Sci Med Sci, 2013. 68(11): p. 1356-76.

249. Szkudelska, K., L. Nogowski, and T. Szkudelski, Resveratrol, a naturally occurring diphenolic compound, affects lipogenesis, lipolysis and the antilipolytic action of insulin in isolated rat adipocytes. J Steroid Biochem Mol Biol, 2009. 113(1-2): p. 17-24. 
250. Mercader, J., A. Palou, and M.L. Bonet, Resveratrol enhances fatty acid oxidation capacity and reduces resistin and Retinol-Binding Protein 4 expression in white adipocytes. J Nutr Biochem, 2011. 22(9): p. 828-34.

251. Rivera, L., et al., Long-term resveratrol administration reduces metabolic disturbances and lowers blood pressure in obese Zucker rats. Biochem Pharmacol, 2009. 77(6): p. 1053-63.

252. Fischer-Posovszky, P., et al., Resveratrol regulates human adipocyte number and function in a Sirt1-dependent manner. Am J Clin Nutr, 2010. 92(1): p. 5-15.

253. Lagouge, M., et al., Resveratrol improves mitochondrial function and protects against metabolic disease by activating SIRT1 and PGC-1alpha. Cell, 2006. 127(6): p. 1109-1122.

254. Szkudelska, K. and T. Szkudelski, Resveratrol, obesity and diabetes. Eur J Pharmacol, 2010. 635(13): p. 1-8.

255. Chen, S., et al., Resveratrol inhibits cell differentiation in 3T3-L1 adipocytes via activation of AMPK. Can J Physiol Pharmacol, 2011. 89(11): p. 793-9.

256. Rayalam, S., et al., Resveratrol induces apoptosis and inhibits adipogenesis in 3T3-L1 adipocytes. Phytother Res, 2008. 22(10): p. 1367-1371.

257. Lasa, A., et al., Resveratrol regulates lipolysis via adipose triglyceride lipase. J Nutr Biochem, 2012. 23(4): p. 379-84.

258. Li, S., et al., Resveratrol inhibits lipogenesis of 3T3-L1 and SGBS cells by inhibition of insulin signaling and mitochondrial mass increase. Biochimica et Biophysica Acta (BBA) - Bioenergetics, 2016. 1857(6): p. 643-652.

259. Kim, S., et al., Resveratrol exerts anti-obesity effects via mechanisms involving down-regulation of adipogenic and inflammatory processes in mice. Biochem Pharmacol, 2011. 81(11): p. 1343-51.

260. Baur, J.A., et al., Resveratrol improves health and survival of mice on a high-calorie diet. Nature, 2006. 444(7117): p. 337-342.

261. Beaudoin, M.S., et al., Resveratrol supplementation improves white adipose tissue function in a depot-specific manner in Zucker diabetic fatty rats. Am J Physiol Regul Integr Comp Physiol, 2013. 305(5): p. R542-51.

262. Zagotta, I., et al., Obesity and inflammation: reduced cytokine expression due to resveratrol in a human in vitro model of inflamed adipose tissue. Front Pharmacol, 2015. 6: p. 79.

263. Rosenow, A., et al., Resveratrol-induced changes of the human adipocyte secretion profile. J Proteome Res, 2012. 11(9): p. 4733-43.

264. Gonzales, A.M. and R.A. Orlando, Curcumin and resveratrol inhibit nuclear factor-kappaBmediated cytokine expression in adipocytes. Nutr Metab (Lond), 2008. 5: p. 17.

265. Fernandez-Quintela, A., et al., Anti-obesity effects of resveratrol: comparison between animal models and humans. J Physiol Biochem, 2016.

266. Yoshino, J., et al., Resveratrol supplementation does not improve metabolic function in nonobese women with normal glucose tolerance. Cell Metab, 2012. 16(5): p. 658-64.

267. Timmers, S., et al., Calorie restriction-like effects of 30 days of resveratrol supplementation on energy metabolism and metabolic profile in obese humans. Cell Metab, 2011. 14(5): p. 612-22.

268. Poulsen, M.M., et al., High-dose resveratrol supplementation in obese men: an investigatorinitiated, randomized, placebo-controlled clinical trial of substrate metabolism, insulin sensitivity, and body composition. Diabetes, 2013. 62(4): p. 1186-95.

269. van der Made, S.M., J. Plat, and R.P. Mensink, Resveratrol does not influence metabolic risk markers related to cardiovascular health in overweight and slightly obese subjects: a randomized, placebo-controlled crossover trial. PLoS One, 2015. 10(3): p. e0118393.

270. Konings, E., et al., The effects of 30 days resveratrol supplementation on adipose tissue morphology and gene expression patterns in obese men. Int J Obes (Lond), 2014. 38(3): p. 470-3.

271. Bhatt, J.K., S. Thomas, and M.J. Nanjan, Resveratrol supplementation improves glycemic control in type 2 diabetes mellitus. Nutr Res, 2012. 32(7): p. 537-41.

272. Brasnyo, P., et al., Resveratrol improves insulin sensitivity, reduces oxidative stress and activates the Akt pathway in type 2 diabetic patients. Br J Nutr, 2011. 106(3): p. 383-9.

273. Timmers, S., et al., Resveratrol as Add-on Therapy in Subjects With Well-Controlled Type 2 Diabetes: A Randomized Controlled Trial. Diabetes Care, 2016. 39(12): p. 2211-2217. 
274. Christenson, J., et al., The Effects of Resveratrol Supplementation in Overweight and Obese Humans: A Systematic Review of Randomized Trials. Metab Syndr Relat Disord, 2016. 14(7): p. 323-33.

275. Calder, P.C., Marine omega-3 fatty acids and inflammatory processes: Effects, mechanisms and clinical relevance. Biochim Biophys Acta, 2015. 1851(4): p. 469-84.

276. Barber, E., A.J. Sinclair, and D. Cameron-Smith, Comparative actions of omega-3 fatty acids on in-vitro lipid droplet formation. Prostaglandins Leukot Essent Fatty Acids, 2013. 89(5): p. 359-66.

277. Murali, G., et al., Differential effects of eicosapentaenoic acid and docosahexaenoic acid in promoting the differentiation of 3T3-L1 preadipocytes. Prostaglandins, Leukotrienes and Essential Fatty Acids, 2014. 90(1): p. 13-21.

278. Polus, A., et al., Influence of dietary fatty acids on differentiation of human stromal vascular fraction preadipocytes. Biochim Biophys Acta, 2015. 1851(9): p. 1146-55.

279. Bertrand, C., et al., Effects of Dietary Eicosapentaenoic Acid (EPA) Supplementation in High-Fat Fed Mice on Lipid Metabolism and Apelin/APJ System in Skeletal Muscle. PLoS ONE, 2013. 8(11): p. e78874.

280. Laiglesia, L.M., et al., Eicosapentaenoic acid promotes mitochondrial biogenesis and beige-like features in subcutaneous adipocytes from overweight subjects. J Nutr Biochem, 2016. 37: p. 7682.

281. Flachs, P., et al., Polyunsaturated fatty acids of marine origin upregulate mitochondrial biogenesis and induce beta-oxidation in white fat. Diabetologia, 2005. 48(11): p. 2365-75.

282. Figueras, M., et al., Effects of Eicosapentaenoic Acid (EPA) Treatment on Insulin Sensitivity in an Animal Model of Diabetes: Improvement of the Inflammatory Status. Obesity, 2011. 19(2): p. $362-$ 369.

283. Kalupahana, N.S., et al., Eicosapentaenoic acid prevents and reverses insulin resistance in high-fat diet-induced obese mice via modulation of adipose tissue inflammation. J Nutr, 2010. 140(11): p. 1915-22.

284. LeMieux, M.J., et al., Eicosapentaenoic acid reduces adipocyte hypertrophy and inflammation in diet-induced obese mice in an adiposity-independent manner. J Nutr, 2015. 145(3): p. 411-7.

285. Clària, J., et al., Pro-resolving actions of SPM in adipose tissue biology. Molecular Aspects of Medicine, 2017. 58: p. 83-92.

286. White, P.J., et al., Protectin DX alleviates insulin resistance by activating a myokine-liver glucoregulatory axis. Nat Med, 2014. 20(6): p. 664-9.

287. Hellmann, J., et al., Resolvin D1 decreases adipose tissue macrophage accumulation and improves insulin sensitivity in obese-diabetic mice. Faseb j, 2011. 25(7): p. 2399-407.

288. Woodman, R.J., et al., Effects of purified eicosapentaenoic and docosahexaenoic acids on glycemic control, blood pressure, and serum lipids in type 2 diabetic patients with treated hypertension. The American Journal of Clinical Nutrition, 2002. 76(5): p. 1007-1015.

289. Tierney, A.C., et al., Effects of dietary fat modification on insulin sensitivity and on other risk factors of the metabolic syndrome--LIPGENE: a European randomized dietary intervention study. Int J Obes (Lond), 2011. 35(6): p. 800-9.

290. Paniagua, J.A., et al., A low-fat high-carbohydrate diet supplemented with long-chain n-3 PUFA reduces the risk of the metabolic syndrome. Atherosclerosis, 2011. 218(2): p. 443-50.

291. Furuhashi, M., et al., Reduction of circulating FABP4 level by treatment with omega-3 fatty acid ethyl esters. Lipids Health Dis, 2016. 15(1): p. 5.

292. Li, K., et al., Effect of marine-derived n-3 polyunsaturated fatty acids on C-reactive protein, interleukin 6 and tumor necrosis factor alpha: a meta-analysis. PLoS One, 2014. 9(2): p. e88103.

293. Itariu, B.K., et al., Long-chain n-3 PUFAs reduce adipose tissue and systemic inflammation in severely obese nondiabetic patients: a randomized controlled trial. Am J Clin Nutr, 2012. 96(5): p. 1137-49.

294. Ahmed, B., S. Liu, and H. Si, Antiadipogenic Effects and Mechanisms of Combinations of Genistein, Epigallocatechin-3-Gallate, and/or Resveratrol in Preadipocytes. J Med Food, 2016.

295. Park, H.J., et al., Combined effects of genistein, quercetin, and resveratrol in human and 3T3-L1 adipocytes. J Med Food, 2008. 11(4): p. 773-83. 
296. Arias, N., et al., The combination of resveratrol and quercetin enhances the individual effects of these molecules on triacylglycerol metabolism in white adipose tissue. European Journal of Nutrition, 2016. 55(1): p. 341-348.

297. Most, J., et al., Short-term supplementation with a specific combination of dietary polyphenols increases energy expenditure and alters substrate metabolism in overweight subjects. Int J Obes (Lond), 2014. 38(5): p. 698-706. 


\section{CHAPTER 2}

\section{Dietary constituents reduce lipid accumulation}

in murine $\mathrm{C} 3 \mathrm{H} 10 \mathrm{~T} 1 / 2$ adipocytes: A novel fluorescent method to quantify fat droplets 


\section{Abstract}

Background: Adipocyte volume (fat accumulation) and cell number (adipogenesis) is increased in obese individuals. Our objective was the identification of dietary constituents with inhibitory effects on triglyceride formation during adipogenesis. Therefore, an in-vitro adipose cell assay in murine C3H10 T1/2 cells was developed, which enabled rapid quantification of intracellular fat droplet accumulation during adipocyte differentiation. Results were corroborated by expression levels of several specific adipogenic and lipogenic genes which are known to regulate triglyceride accumulation.

Methods: $\mathrm{C} 3 \mathrm{H} 10 \mathrm{~T} 1 / 2$ adipocyte differentiation was conducted with rosiglitazone in the presence of test compounds for 7 days. Accumulation of intracellular lipid droplets was measured using the Cellomics ${ }^{\circledR}$ ArrayScan ${ }^{\circledR}$ VTI HCS reader and SpotDetector ${ }^{\circledR}$ BioApplication from ThermoFisher. Fluorescent images were automatically acquired and analysed employing the fluorescent dyes BODIPY ${ }^{\circledR} 493 / 503$ and Hoechst 33342 , for staining neutral lipids and localisation of nuclei, respectively. The expression levels of adipogenic and lipogenic genes, such as PPAR $\alpha$ and PPAR $\gamma, \mathrm{C} / \mathrm{EBP} \alpha, \mathrm{aP} 2$, adiponectin, LPL and HSL, CPT-1 $\beta$, ACC1, Glut4 and FAS, were determined by quantitative RT-PCR. Dietary ingredients including PUFAs, carotenoids, polyphenols and catechins were tested for their effect on lipid accumulation.

Results: The $\omega-3$ PUFAs docosahexaenoic acid (DHA) and eicosapentaenoic acid (EPA), the carotenoid $\beta$-carotene and hydroxytyrosol exhibited the strongest inhibitory effects on the rosiglitazone-stimulated lipid formation. (all-E)-lycopene and epigallocatechin gallate (EGCG) showed a moderate inhibition, whereas resveratrol did not reduce fat droplet formation. Additionally, it was demonstrated that adipogenic and lipogenic gene expression was attenuated. DHA, $\beta$-carotene and hydroxytyrosol inhibited the gene expression of PPAR $\gamma, \mathrm{C} / \mathrm{EBP} \alpha, \mathrm{aP} 2$ and $\mathrm{CPT}-1 \beta$.

Conclusion: This in-vitro assay in differentiating adipocytes enables automated detection and quantification of changes in lipid droplet number, size, and intensity. The observed inhibitory effects of identified dietary constituents such as $\omega-3$ PUFAs and $\beta$-carotene correlate with the modulation of genes involved in adipocyte differentiation. 


\section{Background}

The metabolic disorder obesity leads to various diseases such as hypertension, type-2-diabetes, respiratory complications, and coronary heart disease [1]. This accounts for the numerous studies on cellular and molecular processes underlying fat metabolism in recent years [2, 3].

Adipocytes are specialised cells that store triacylglycerides (TGs) in times of energy excess and release energy by lipolysis during energy shortage [2]. A constant positive energy balance leads to an excessive fat accumulation in white adipose tissue (WAT). Two mechanisms make this possible: (1) hypertrophy of adipocytes and (2) hyperplasia of proliferating pre-adipocytes into differentiated adipocytes [4]. This complex process called adipogenesis is sequentially regulated by several transcription factors such as peroxisome proliferator-activated receptor gamma (PPAR $\gamma$ ) [5], CCAAT/enhancer binding proteins (C/EBP $\alpha, \mathrm{C} / \mathrm{EBP} \beta$ and $\mathrm{C} / \mathrm{EBP} \delta)[6]$ and the adipocyte determination and differentiation factor 1 (ADD1/SREBP-1c) [3]. However, WAT is also an endocrine tissue that secretes metabolically active substances (adipokines), which function as feedback signals or lead to immunological responses [2].

The inhibition of differentiation of pre-adipocytes into adipocytes may regulate the amount of adipose tissue [7]. This has triggered the discovery of pharmacological inhibitors of adipogenesis and intensified the search for dietary ingredients with similar properties [8]. Food constituents such as polyphenols or carotenoids are dietary substances that are precursors of, or function as, signalling molecules. Most of these substances are plant-derived, being present in fruits, vegetables, and nuts; likewise, polyunsaturated fatty acids (PUFAs) in fish and algae have similar properties. Many food ingredients have been described as modulators of adipocyte differentiation e.g. dietary PUFAs (for review see Madsen et al. [9]).

Several cell models exist to simulate differentiation of pre-adipocytes in-vitro, the most widelyused being the 3T3-L1 cell line [10] derived from 3T3 cells [11]. Another commonly-employed model is the multipotent embryonic fibroblast cell line C3H10 T1/2 [12]. Pre-adipocyte differentiation is assessed through visualisation of accumulated fat droplets via neutral lipid staining. Alternatively, late adipocyte differentiation markers and genes related to lipid metabolism, such as lipoprotein lipase (LPL) [13], adipocyte fatty acid binding protein (aP2) [14], fatty acid synthase (FAS) [15], hormone-sensitive lipase (HSL) [16] or carnitine palmitoyltransferase 1 (CPT-1), can be measured.

The identification of bioactive compounds that might reduce excessive WAT requires valid invitro test systems that allow for the investigation of a greater number of compounds and rapid quantitative detection of relevant fat cell differentiation features. 
The main objective of the present investigation was to identify the effects of food constituents that could modulate the differentiation of $\mathrm{C} 3 \mathrm{H} 10 \mathrm{~T} 1 / 2$ cells into mature adipocytes and the concomitant accumulation of cytosolic TGs. For this purpose, we have developed a new morphological, high content, cell assay (HCA) using the Cellomics ${ }^{\circledR}$ ArrayScan ${ }^{\circledR}$ VTI HCS Reader and the SpotDetector ${ }^{\circledR}$ BioApplication software from ThermoFisher. Several chemical classes of dietary ingredients, such as PUFAs, carotenoids, polyphenols and catechins, were tested in this assay. Furthermore, we examined the effects of these compounds on expression levels of genes known to play key roles in adipocyte differentiation and fat metabolism. Our data demonstrate that the HCA assay is a valuable substitute for the commonly-used Oil Red O procedure [17], enabling improved in situ quantification of fat droplet number, size and intensity in adipocytes.

\section{Methods}

\section{Materials}

Dulbecco's modified Eagle's cell culture medium (DMEM), penicillin G (10000 units/ml) and streptomycin $(10000 \mu \mathrm{g} / \mathrm{ml})$ solution (pen/strep), L-glutamine $(100 \mathrm{x}, 200 \mathrm{mM})$ and phosphatebuffered saline without $\mathrm{Ca}^{2+}$ and $\mathrm{Mg}^{2+}$ (PBS (-/-)) were from GIBCO ${ }^{\mathrm{TM}}$ (Grand Island, NY, USA). Fetal calf serum (FCS) and trypsin were purchased from AMIMED ${ }^{\circledR}$ (BioConcept, Allschwil, Switzerland). FALCON ${ }^{\circledR}$ culture flasks were from BECTON DICKINSON (Franklin Lakes, NJ, USA) and 24-well plates coated with collagen type I were supplied by Greiner bio-one (Frickenhausen, Germany).

Rosiglitazone was purchased from Shanco International (NJ, USA) and recombinant bovine insulin (dissolved in $\mathrm{H}_{2} \mathrm{O}$ ), resveratrol and $\omega$-3 PUFAs, docosahexaenoic acid (DHA) and eicosapentaenoic acid (EPA), were from Sigma-Aldrich (St. Louis, MO, USA). 3-hydroxytyrosol (HT) was from Cayman Chemicals (Ann Abor, MI, USA). Epigallocatechin gallate (EGCG, Teavigo $^{\mathrm{TM}}$ ) and carotenoids were supplied by DSM Nutritional Products Ltd (Basel, Switzerland). Carotenoids were dissolved in tetrahydrofurane (THF). All other test compounds were dissolved in dimethylsulfoxide (DMSO).

Fluorescent dyes - Hoechst 33342 and BODIPY ${ }^{\circledR}$ 493/503 - were obtained from Molecular Probes $^{\mathrm{TM}}$ (Eugene, OR, USA). The CytoTox ${ }^{\circledR}$ non-radioactive cytotoxicity assay kit was purchased from Promega (Fitchburg, WI, USA). Primers and probes were synthesised by Applied Biosystems (PE Biosystems, Foster City, CA, USA) or by Sigma Genosys (St. Louis, MO, USA). 


\section{Cell culture}

Mouse embryonic fibroblast cells C3H10 T1/2 [12] were from ATCC-LGC (Middlesex, UK) and cultured according to the supplier's protocol. Briefly, cells were grown in high glucose $(4.5 \mathrm{~g} / \mathrm{ml})$ DMEM supplemented with $10 \%$ FCS and $0.5 \%$ pen/strep (v/v) (growth medium; GM) at $37^{\circ} \mathrm{C}$ and $5 \% \mathrm{CO}_{2}$. Cells were cultured until they reached $80-90 \%$ confluence; they were used between passage 6 and 20 for the experiments described below.

\section{Adipocyte differentiation assay}

$\mathrm{C} 3 \mathrm{H} 10 \mathrm{~T} 1 / 2$ cells were plated $\left(2 \times 10^{5}\right.$ cells $\left./ \mathrm{cm}^{2}\right)$ in collagen-I-coated 24 -well plates and maintained in phenol red-free GM supplemented with $2 \mathrm{mM}$ L-glutamine (GM1) until 1 day postconfluence (day 0). Differentiation medium (DM) containing $200 \mathrm{nM}$ bovine insulin and $10 \mu \mathrm{M}$ rosiglitazone was then added [18]. C3H10 T1/2 pre-adipocytes were either cultured in DM only (positive control) or in DM supplemented with different doses of test compounds $(0.5-25 \mu \mathrm{M})$ for 4 days (gene expression study) or 7 days. Each treatment was performed in triplicate. The final DMSO and/or THF concentration was adjusted for all treatments and controls to $0.2 \%$ and/or $0.1 \%$, respectively, and medium and compounds were renewed every other day. Cells were lysed after $96 \mathrm{~h}$ or, after 1 week treatment, fixed with $60 \%$ isopropanol for $1.5 \mathrm{~h}$ at $4{ }^{\circ} \mathrm{C}$ and stained (see below).

\section{Staining procedures}

Adipocytes were stained with Oil Red O or with the fluorescent dyes Hoechst 33342 (Hoe) and BODIPY ${ }^{\circledR} 493 / 503$ (BP). In brief, fixed cells were first washed twice with PBS (-/-). For the Oil Red O staining, cells were incubated with $200 \mu \mathrm{l}$ of Oil Red O solution $(0.33 \% \mathrm{w} / \mathrm{v}$ in $60 \%$ isopropanol) for $20 \mathrm{~min}$ at room temperature (RT). The fluorescent staining comprised addition of $200 \mu \mathrm{l} \mathrm{Hoe} / \mathrm{BP}$ solution $(3 \mu \mathrm{g} / \mathrm{ml}$ Hoe, $0.75 \mu \mathrm{g} / \mathrm{ml}$ BP in PBS (-/-)) per well, followed by incubation in the dark at RT for $30 \mathrm{~min}$. Before analysis, the cells were washed twice with PBS ($/-)$.

\section{Visualisation of Oil Red $O$ stained adipocytes and quantification of Oil Red 0}

Images of Oil Red O stained adipocytes were acquired using a Nikon Coolpix 990 camera at 20x magnification and further edited with Adobe ${ }^{\circledR}$ Photoshop ${ }^{\circledR}$ CS2 version 9.

To quantify staining of fat droplets, Oil Red O stain was extracted by adding isopropanol to each well immediately after removal of the wash solution $(300 \mu \mathrm{l}$ per well). Plates were incubated for $10 \mathrm{~min}$ at RT; two aliquots of $100 \mu \mathrm{l}$ were transferred to 96-well plates and read immediately at $510 \mathrm{~nm}$ (Spectra Max Plus 384, Molecular Devices, CA USA). After subtraction of the 
background value (wells without cells, but treated with medium; non-specific binding), the differentiation of treated cells was calculated as percent of positive control.

\section{Automated determination of lipid accumulation in adipocytes}

Lipid droplets were quantified with the Cellomics ${ }^{\circledR}$ ArrayScan ${ }^{\circledR}$ VTI HCS Reader (500series, version 5.6.1.4 - 0.63x, ThermoFisher Scientific). Fat droplets were detected with the provided SpotDetector ${ }^{\circledR}$ BioApplication. The fluorescent dye, BODIPY ${ }^{\circledR} 493 / 503$, was used to visualise and quantify fat droplets (number, area, intensity) in differentiated C3H10 T1/2 adipocytes, while nuclei were stained using the Hoechst 33342 dye. The method is based on a two-channel assay, which uses a 40x objective (NA 0.5), a Hamamatsu ORCA-ER digital camera in combination with a $0.63 x$ coupler and Carl Zeiss microscope optics for image acquisition. Images were acquired in high resolution $(1024 \times 1024,1 \times 1)$ and auto focus mode $(1024 \times 1024,4 \times 4)$ resulting in a field width of 262 microns. Channel one (Ch1) applies the XF93-Hoechst filter and is the focus channel in which objects (nuclei) are identified, and the spots (fat droplets) are detected in channel two (Ch2, XF93-FITC filter).

The default settings of the SpotDetector.V2 assay algorithm version 4.1 were altered such that, in $\mathrm{Ch} 2$, thresholds were set to ensure that only fat droplets of a certain size and intensity were selected for analysis of 100 fields per well (Table 2.1). The data of each channel were reported on a "per field" basis.

\section{Cell viability}

Cell viability was assessed using the CytoTox $^{\circledR}$ non-radioactive cytotoxicity assay kit (lactate dehydrogenase (LDH) determination) and by calculating the relative cell number according to the following equation: cell number $(\%)=$ (average number of cells per fields [treatment] * 100 / average number of cells per fields [control]). Cytotoxic effects of the compounds were rated by comparison of the LDH levels in the supernatants of compound-treated and untreated cells at day 7. Nuclei were counted with the ArrayScan ${ }^{\circledR}$ reader. 
Table 2.1: Protocol parameters differing from the standard SpotDetector ${ }^{\circledR}$ algorithm

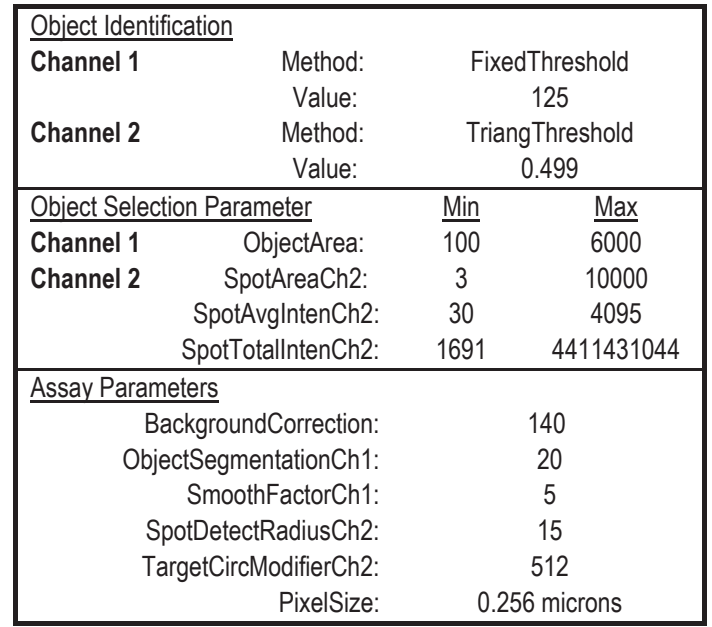

The alteration of assay parameters enables adjustment of the algorithm to different biological cell models. Displayed values are pixel number.

\section{Gene expression study}

Total mRNA was extracted using the RNeasy ${ }^{\circledR} 96$ Kit (Qiagen, Hilden, Germany) and quantified with the RiboGreen ${ }^{\circledR}$ Kit (Invitrogen, Molecular Probes ${ }^{\mathrm{TM}}$, Eugene, OR, USA) according to the manufacturer's protocols. RNA quality was assessed using RNA 6000 Nano Chips for the Agilent 2100 Bioanalyzer (Agilent Technologies, USA). For first strand cDNA synthesis $650 \mathrm{ng}$ total RNA was reverse-transcribed using the Omniscript ${ }^{\circledR}$ RT Kit from Qiagen (20 $\mu 1$ reaction mix), random primers from Promega and RNaseOUT ${ }^{\mathrm{TM}}$ from Invitrogen. After three incubation steps $\left(60 \mathrm{~min}\right.$ at $37^{\circ} \mathrm{C}, 5 \mathrm{~min}$ at $93^{\circ} \mathrm{C}, 5 \mathrm{~min}$ at $4^{\circ} \mathrm{C}$ ), cDNA solutions were diluted with DEPC-treated water to $260 \mu \mathrm{l}$ and stored at $-20^{\circ} \mathrm{C}$.

Quantitative TaqMan ${ }^{\text {TM }}$ RT-PCR was performed using an ABI-PRISM ${ }^{\circledR} 7900$ HT Sequence Detection System (PE Biosystems, Foster City, CA, USA) and MicroAmp ${ }^{\circledR}$ Optical 96-well reaction plates (PE Biosystems, Foster City, CA, USA). Briefly, $5 \mu 1$ cDNA was added to $20 \mu 1$ reaction mixture, containing 1× Universal Master Mix (PE Biosystems, Rotkreuz, Switzerland), $300 \mathrm{nM}$ PCR primers (forward and reverse), and $100 \mathrm{nM}$ TaqMan ${ }^{\mathrm{TM}}$ probe (FAM-TAMRA) for the gene of interest. 18S rRNA was used as endogenous control (EC), with primers and probes (VIC-TAMRA) at $50 \mathrm{nM}$ and $100 \mathrm{nM}$, respectively. Primers and probes were designed using the Primer Express software (Applied Biosystems, Forster City, CA, USA). The oligonucleotide sequences for the primers and probes are shown in Table 2.2. The cycle conditions were: 2 min at $50^{\circ} \mathrm{C}, 10 \mathrm{~min}$ at $95^{\circ} \mathrm{C}, 40$ cycles of $15 \mathrm{sec}$ at $95^{\circ} \mathrm{C}$ and $60 \mathrm{sec}$ at $60^{\circ} \mathrm{C}$. Threshold $\mathrm{C}_{\mathrm{T}}$ values were set at 0.05 . Baseline start and stop values for the gene of interest were set at 3 and 15 , respectively, 
and for $18 \mathrm{~S}$ rRNA at 3 and 7, respectively. mRNA abundance was calculated using the comparative $\mathrm{C}_{\mathrm{T}}$ method according to the manufacturer's protocol. Shortly, $\Delta \mathrm{C}_{\mathrm{T}}=\mathrm{C}_{\mathrm{T}}$ [gene of interest $]-\mathrm{C}_{\mathrm{T}}[E C]$ and $\Delta \Delta \mathrm{C}_{\mathrm{T}}=\Delta \mathrm{C}_{\mathrm{T}}[$ rosiglitazone control cells $]-\Delta \mathrm{C}_{\mathrm{T}}[$ treated cells $]$. The fold expression for the gene of interest was expressed as $2^{-\Delta \Delta \mathrm{C}_{\mathrm{T}}}$.

Table 2.2: Sequences of primers and probes for different adipocyte specific genes used in real-time RT-PCR

\begin{tabular}{|c|c|c|}
\hline Gene name & $\begin{array}{l}\text { Forward Primer } \\
\text { Reverse Primer }\end{array}$ & Probe \\
\hline Adiponectin (Acrp30) & $\begin{array}{l}\text { 5'-GGCACTCCTGGAGAGAAGGG } \\
\text { 5'-ATTCCAACATCTCCTGTCTCACC }\end{array}$ & 5'-GAAAGGAGATGCAGGTCTTCTTGGTCCTA \\
\hline $\begin{array}{l}\text { Fatty acid binding protein } 4 \text {, adipocyte } \\
\text { type (aP2) }\end{array}$ & $\begin{array}{l}\text { 5'-GCGTGGAATTCGATGAAATCA } \\
\text { 5'-CCCGCCATCTAGGGTTATGA }\end{array}$ & 5'-CGCAGACGACAGGAAGGTGAAGAGC \\
\hline $\begin{array}{l}\text { Acetyl-CoA carboxylase } \\
\text { (ACC1) }\end{array}$ & $\begin{array}{l}\text { 5'-TCACTCGCTTTGGAGGCAA } \\
\text { 5'-CGCAGCGATGCCATTGT }\end{array}$ & 5'-AGGGTCATAGAGAAGGTGCTCATCGCC \\
\hline Lipoprotein lipase (LPL) & $\begin{array}{l}\text { 5'-GTGGCCGAGAGCGAGAAC } \\
\text { 5'-AAGAAGGAGTAGGTTTATTTGTGGAA }\end{array}$ & 5'-TTCCCTTCACCCTGCCCGAGG \\
\hline $\begin{array}{l}\text { Hormone sensitive lipase } \\
\text { (HSL) }\end{array}$ & $\begin{array}{l}\text { 5'-AAGACCACATCGCCCACAG } \\
\text { 5'-CAGACACACTCCTGCGCATAG }\end{array}$ & 5'-AGAGTCTGTGCGCCCCACGGA \\
\hline Fatty acid synthase (FAS) & $\begin{array}{l}\text { 5'-TCATAAAGCAGTTTCTTGATGTGGA } \\
\text { 5'-CAGGCTCTTCAGTGGCAGC }\end{array}$ & 5'-CACAGCAAGGTGCTGGAGGCCC \\
\hline $\begin{array}{l}\text { Carnitine palmitoyl } \\
\text { transferase beta (CPT-1 } \beta \text { ) }\end{array}$ & $\begin{array}{l}\text { 5'-CCAATCATCTGGGTGCTGG } \\
\text { 5'-TAAGAGACCCCGTAGCCATCAT }\end{array}$ & 5'-TGGCTTTGGTCCCGTGGCG \\
\hline $\begin{array}{l}\text { Glucose transporter } 4 \\
\text { (Glut4) }\end{array}$ & $\begin{array}{l}\text { 5'-TCGGCTCTGACGATGGG } \\
\text { 5'-CCAAGCCAGCTGAGAATACAG }\end{array}$ & 5'AACCCCCTCGGCAGCGAGTGACTG \\
\hline $\begin{array}{l}\text { Peroxisome proliferator activated } \\
\text { receptor gamma 2a (PPAR } 2 \mathrm{2a} \text { ) }\end{array}$ & $\begin{array}{l}\text { 5'-CTATGAGCACTTCACAAGAAATTACCAT } \\
\text { 5'-TCCATCACGGAGAGGTCCAC }\end{array}$ & 5'- TCTGGCCCACCAACTTCGGAATCAG \\
\hline $\begin{array}{l}\text { Peroxisome proliferator activated } \\
\text { receptor alpha (PPAR } \alpha \text { ) }\end{array}$ & $\begin{array}{l}\text { 5'-GCCTCAGGGTACCACTACGG } \\
\text { 5'-GCCGAATAGTTCGCCGAA }\end{array}$ & 5'-CACGCATGTGAAGGCTGTAAGGGCTT \\
\hline $\begin{array}{l}\text { CCAAT/enhancer binding protein alpha } \\
(\mathrm{C} / E B P \alpha)\end{array}$ & $\begin{array}{l}\text { 5'-CGGTGCGGGCAAAGC } \\
\text { 5'-TGCGTTCCCGCCGTAC }\end{array}$ & 5'-AGAAGTCGGTGGACAAGAACAGCAAC \\
\hline 18S rRNA & $\begin{array}{l}\text { 5'-CGGCTACCACATCCAAGG } \\
\text { 5'-CGGGTCGGGAGTGGGT }\end{array}$ & 5'-TTGCGCGCCTGCTGCCT \\
\hline
\end{tabular}

\section{Statistical analysis}

Statistical significance of the mean differences between treatment and positive control was tested by Student's t-test for unpaired values. P values less than 0.05 were considered significant. ArrayScan ${ }^{\circledR}$ results are shown as mean \pm SEM. Gene expression data are expressed as fold change \pm error (based on SEM). 


\section{Results}

\section{Cross-validation of Cellomics $₫$ assay with Oil Red 0 method}

For validation of the Cellomics ${ }^{\circledR}$ assay, the established Oil Red O method was used as reference, and the accumulation of intracellular lipid droplets was measured with both methods. C3H10 T1/2 cells were differentiated for 7 days using the PPAR $\gamma$ agonist rosiglitazone, at concentrations between 0.01 and $100 \mu \mathrm{M}$. Cells grown in DM without rosiglitazone (negative control) accumulated only a few small fat droplets. The average number of fat droplets per cell increased from 0.5 (negative control) to 4.0 (positive control). The fat droplets were both significantly brighter and larger $(\mathrm{p}<0.001)$ in cells incubated with $10 \mu \mathrm{M}$ rosiglitazone.

To assess the quality and suitability of the new methodology the assay windows (positive control (p)/negative control (n)) and the estimated Z-factors (1- $\left(\left(3 \times\left(\sigma_{p}+\sigma_{n}\right)\right) /\left(\left|\mu_{p}-\mu_{n}\right|\right) ; \mu=\right.$ sample means and $\sigma=$ sample standard deviation) for the different assay parameters were determined. A $\mathrm{Z}$-value between 0.5 and 1 is interpreted as an exceptional assay, whereas a value between 0 and 0.5 is indicative of a marginal assay [19]. The Oil Red O method had an assay window of 2.3 and a Z-factor of only -0.08 ; where $0.2 \%$ DMSO was used as negative control and $100 \mu \mathrm{M}$ rosiglitazone as maximum positive control. In contrast, the Cellomics ${ }^{\circledR}$ assay resulted in a window of 6.8 and a Z-factor of 0.71 for the Spot Count / Object parameter. During screening, an assay window greater than 20 and an average Z-value of 0.65 was achieved with $10 \mu \mathrm{M}$ rosiglitazone as positive control and $0.2 \% \mathrm{DMSO}$ as negative control, indicating that the fluorescent method is a robust assay (CV Spot Count / Object = 0.09) [19].
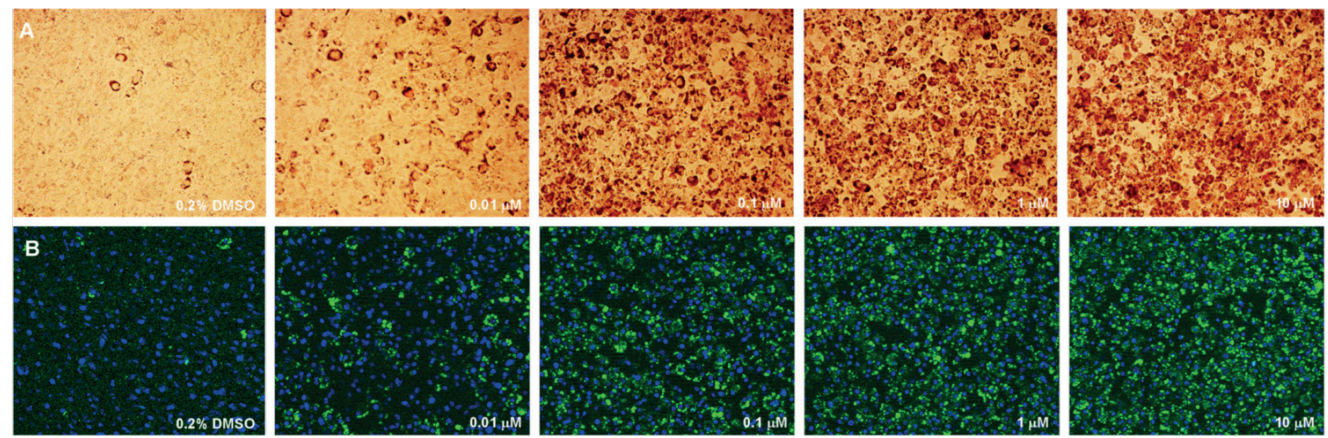

Figure 2.1: Visualisation of lipid accumulation in differentiated adipocytes

C3H10 T1/2 cells were treated for 7 days with rosiglitazone $(0.01,0.1,1$ and $10 \mu \mathrm{M})$ and, after fixation with $60 \%$ isopropanol, stained with (A) Oil Red O or (B) Hoechst 33342 and BODIPY® 493/503. Images were acquired using A) a Nikon Coolpix 990 camera at $20 x$ magnification or B) the Cellomics ${ }^{\circledR}$ HCS Reader camera (20x). 
Rosiglitazone dose-dependently increased the number of Oil Red O-stained lipid droplets (Figure 2.1A). To quantify the microscopic findings, Oil Red $\mathrm{O}$ was eluted from the fat droplets and measured spectro-photometrically (Figure 2.2A). A differentiation plateau seemed to be reached between $10 \mu \mathrm{M}$ and $100 \mu \mathrm{M}$ rosiglitazone, hence $100 \mu \mathrm{M}$ values were used as references for calculations. The fluorescent stain BODIPY ${ }^{\circledR}$ likewise enabled measurement of rosiglitazonedependent induction of TG formation (Figure 2.1B). Both fat droplet number (Spot Count / Object) and fat droplet intensity (Spot Avg Intensity) concentration-dependently increased with rosiglitazone (Figure 2.2B). Thus, our method proved valid for detecting intracellular lipid accumulation.
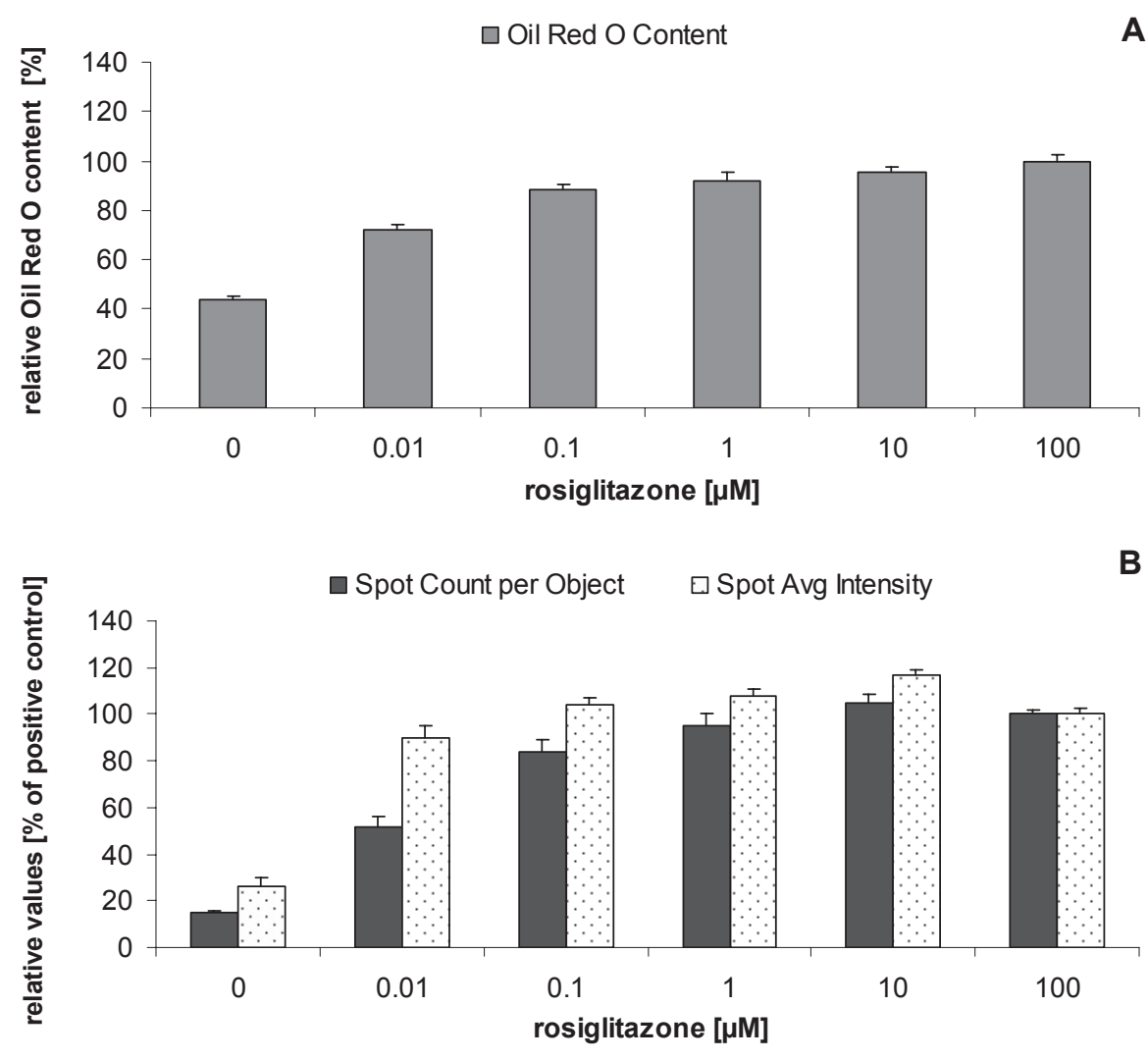

Figure 2.2: Determination of intracellular fat content in adipocytes differentiated for 7 days with varying concentrations of rosiglitazone

(A) Oil Red O quantification according to the Materials and Methods section. (B) Fat droplet number (Spot Count / Object) and droplet intensity (Spot Avg Intensity), using the Cellomics HCS Reader. Neutral lipids were stained with BODIPY® 493/503. Results are expressed as percentage of values obtained with $100 \mathrm{aM}$ rosiglitazone (mean $\pm S E M, n=8$ ). 


\section{Effects of substances on number of maturing $\mathrm{C} 3 \mathrm{H} 10 \mathrm{~T} 1 / 2$ cells}

Cell (or nuclei) number was considered to reflect cell viability. Cells treated with different concentrations of DHA, EPA, EGCG, HT, resveratrol, (all-E)-lycopene and $\beta$-carotene had a similar nuclei count as control cells. The number of cells was slightly reduced $(<20 \%)$ by incubation with EGCG, HT, resveratrol, and $\beta$-carotene at the highest concentration (data not shown). This was corroborated by the measurement of LDH activity in supernatants after 7 days' treatment. Only DHA and EPA increased the LDH release at $25 \mu \mathrm{M}$ (data not shown). The data indicate that cell viability was not impaired by the substances, although some of them might have apoptotic effects at high concentrations.

\section{PUFAs and $\beta$-carotene inhibit lipid accretion in maturing adipocytes}

Subsequently, the effects of test substances on lipid accumulation in adipocytes were investigated using the Cellomics ${ }^{\circledR}$ method. Murine pre-adipocytes were differentiated for 7 days $(n=10)$ in the absence or presence of $\omega-3$ PUFAs (DHA or EPA), carotenoids, (poly)-phenols, and catechins. Incubation of rosiglitazone-treated C3H10 T1/2 cells with $25 \mu \mathrm{M}$ DHA, $25 \mu \mathrm{M}$ EPA, or $2 \mu \mathrm{M} \beta$ carotene, significantly decreased the number of lipid droplets (Spot Count / Object) by $56 \%, 42 \%$ and $41 \%$, respectively (Figure 2.3 ).

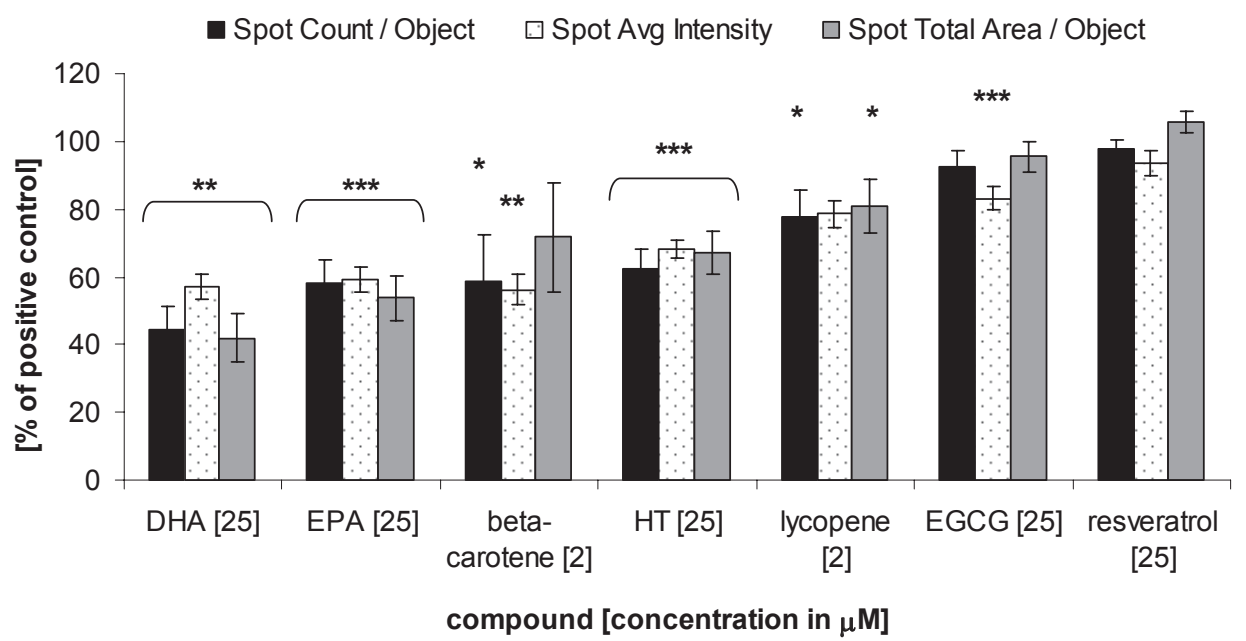

Figure 2.3: Effects of DHA, EPA, $\beta$-carotene, HT, (all-E)-lycopene, EGCG and resveratrol on lipid formation measured with the HCA assay

C3H10 T1/2 cells were treated for 7 days. Shown are three parameters (Spot Count / Object, Spot Avg Intensity, and Spot Total Area / Object) that quantify lipid formation, as compared to rosiglitazone controls set as $100 \%$. Data are shown as mean \pm SEM $(\mathrm{n}=10-20)$. Student's t-test: treatment versus control $\left.\left(^{*}\right) p<0.05,\left({ }^{* *}\right) p<0.01,{ }^{(* *}\right) p<0.001$. 
At $25 \mu \mathrm{M}$ HT inhibited the accumulation of TGs in adipocytes by $38 \%$, whereas (all-E)-lycopene and EGCG reduced the fat droplets by 22 and 7\%, respectively. The test compounds also lowered the spot intensity (Spot Avg Intensity) and Spot Total Area / Object parameters to a similar extent, suggesting that the total fat content per cell was reduced. Compared to the PUFAs, resveratrol did not affect adipogenesis and even increased TG content.

\section{Concentration-dependent effects of test compounds on TG accumulation}

In order to investigate the concentration-response relationship of the test substances, we differentiated pre-adipocytes for 7 days in the presence of 3 concentrations of each test compound. DHA and EPA concentration-dependently reduced both fat droplet number and intensity (Figure 2.4). $\beta$-carotene showed a more potent concentration-dependency than (all-E-)-lycopene. EGCG and resveratrol, however, had no inhibitory effect on intracellular TG accumulation (except EGCG at $25 \mu \mathrm{M}$; Figure 2.3). In contrast, HT showed a similar concentration-dependent modification of fat droplets as EPA (data not shown).

\section{Reduced expression of genes involved in adipocyte differentiation and in glucose and fatty acid metabolism}

To explore a possible impact of the tested substances at the transcriptional level, we differentiated pre-adipocytes with $10 \mu \mathrm{M}$ rosiglitazone in the absence or presence of DHA, EPA, EGCG, HT, resveratrol, (all-E-)-lycopene or $\beta$-carotene and assessed gene expression levels by quantitative RT-PCR. Tested genes were the master regulators of adipogenesis PPAR $\gamma$ and C/EBP $\alpha$, the nuclear receptor PPAR $\alpha$, as well as the adipocyte differentiation markers fatty acid binding protein 2 (aP2) and adipose tissue-specific adiponectin. mRNA levels of genes involved in fatty acid metabolism (ACC1, CPT-1 $\beta$, FAS, HSL, LPL) and glucose uptake (Glut4) were also determined. Treatment with DHA $(25 \mu \mathrm{M})$ reduced the expression of aP2 and adiponectin, to $48 \%$ and $59 \%$ respectively (Table 2.3). The expression of aP2 and adiponectin after treatment with $2 \mu \mathrm{M} \beta$ carotene was also significantly decreased to $31 \%$ and $39 \%$, and at $25 \mu \mathrm{M}$ HT to $47 \%$ and $69 \%$, respectively (Figure 2.5, Table 2.3). The mRNA levels of the adipogenesis-associated transcription factors, PPAR $\gamma$, PPAR $\alpha$ and C/EBP $\alpha$ were strongly suppressed by PUFAs, $\beta$-carotene and HT at the highest concentration (up to 85\%; Table 2.3). Although PPAR $\gamma$ and C/EBP $\alpha$ were suppressed by $\beta$-carotene, as by DHA and EPA, not exactly the same down-stream genes were affected. Interestingly $1 \mu \mathrm{M}$ DHA led to a significant increase in the mRNA level of $\mathrm{C} / \mathrm{EBP} \alpha$. 

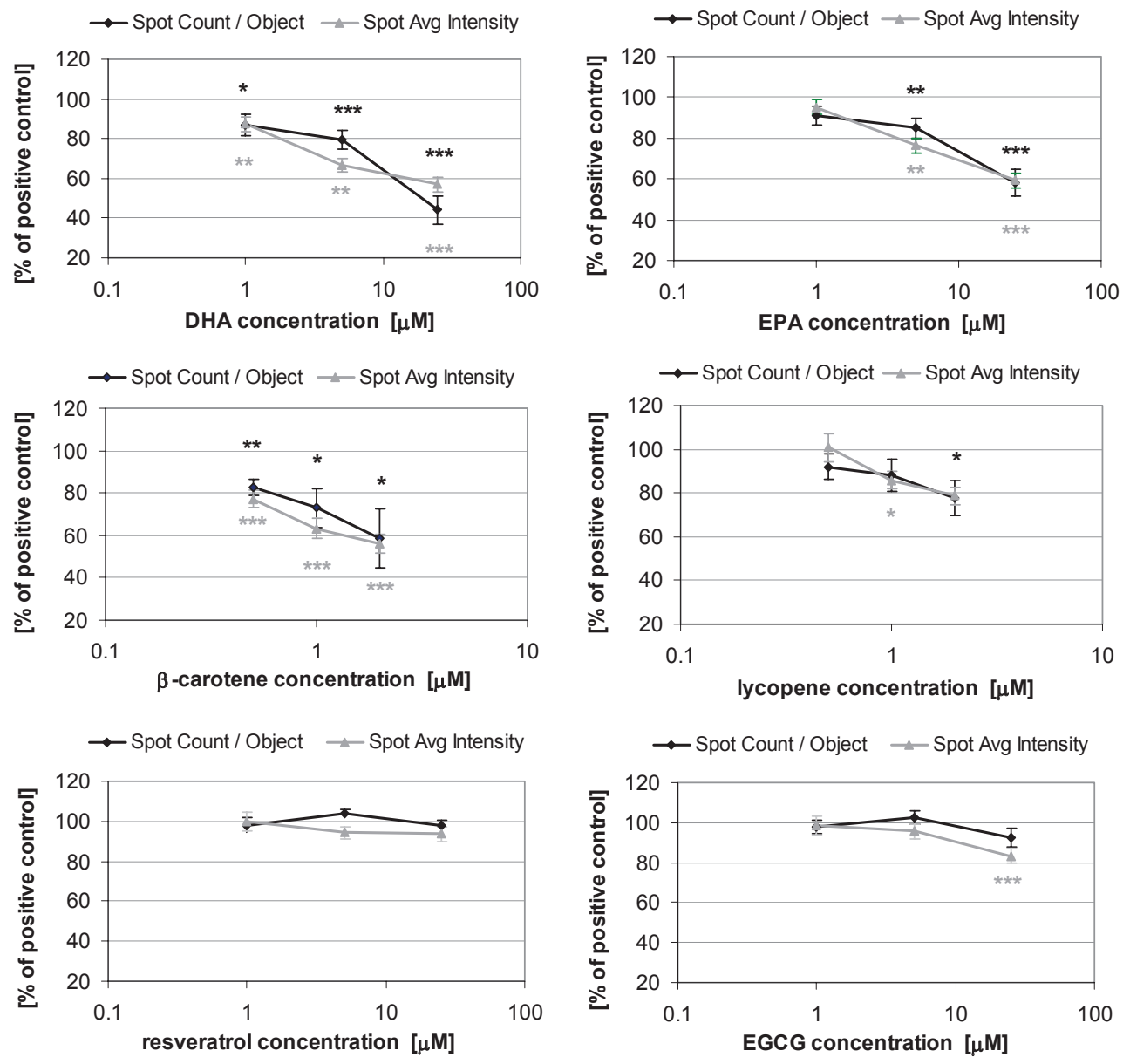

Figure 2.4: Effects of varying concentrations of DHA, EPA, $\beta$-carotene, (all-E)-lycopene, resveratrol, and EGCG on number and intensity of fat droplets

C3H10 T1/2 cells were differentiated in the presence of test substances for 7 days. Spot Count / Object and Spot Avg Intensity were determined, as compared to rosiglitazone control set as 100\%. Data are shown as mean \pm SEM $(n=10-20)$. Student's t-test: treatment versus control $\left({ }^{*}\right) p<0.05,\left(^{* *}\right) p<0.01,\left({ }^{* *}\right) p<0.001$.

ACC1, CPT-1 $\beta$, FAS, HSL and LPL are enzymes which control triglyceride transport, fatty acid synthesis and transport of long-chain fatty acids in differentiated fat cells. In the case of CPT-1 $\beta$, its expression was significantly reduced by DHA to $21 \%$ and by HT to $44 \%$, but not by lycopene (Table 2.3). The effects of DHA and $\beta$-carotene on gene expression levels were concentrationdependent (Figure 2.5). LPL mRNA levels were strongly diminished to $10 \%$ by all $\beta$-carotene concentrations. 
Table 2.3: Effects of compounds on gene expression of adipocyte differentiation markers and enzymes involved in fat and glucose metabolism $(n=6-15)$

\begin{tabular}{|c|c|c|c|c|c|c|c|c|}
\hline & & \multicolumn{2}{|c|}{ PUFAs } & \multicolumn{2}{|c|}{ CAROTENOIDS } & \multicolumn{3}{|c|}{ PHENOLS } \\
\hline & & $\frac{\mathrm{DHA}}{25 \mu \mathrm{M}}$ & $\frac{\text { EPA }}{25 \mu \mathrm{M}}$ & $\frac{\text { lycopene }}{2 \mu \mathrm{M}}$ & $\frac{\beta \text {-carotene }}{2 \mu \mathrm{M}}$ & $\frac{E G C G}{25 \mu M}$ & $\frac{\mathrm{HT}}{25 \mu \mathrm{M}}$ & $\frac{\text { Resv }}{25 \mu \mathrm{M}}$ \\
\hline \multirow[t]{3}{*}{ PPAR $\gamma 2$} & fold & 0.48 & 0.61 & 0.93 & 0.48 & 0.97 & 0.53 & 1.07 \\
\hline & error $+1-$ & $0.11 / 0.09$ & $0.09 / 0.08$ & $0.33 / 0.25$ & $0.17 / 0.13$ & $0.20 / 0.17$ & $0.15 / 0.12$ & $0.12 / 0.11$ \\
\hline & $p$-value & $<0.001$ & 0.002 & 0.75 & 0.04 & 0.64 & $<0.001$ & 0.53 \\
\hline \multirow[t]{3}{*}{ PPAR $\alpha$} & fold & 0.15 & 0.25 & 1.01 & 0.80 & 0.81 & 0.47 & 0.92 \\
\hline & error +1 - & $0.10 / 0.06$ & $0.10 / 0.07$ & $0.57 / 0.36$ & $0.37 / 0.25$ & $0.11 / 0.09$ & $0.15 / 0.12$ & $0.07 / 0.07$ \\
\hline & $p$-value & $<0.001$ & $<0.001$ & 0.94 & 0.69 & 0.33 & $<0.001$ & 0.99 \\
\hline \multirow[t]{3}{*}{$\mathrm{C} / \mathrm{EBP} \alpha$} & fold & 0.60 & 0.82 & 0.86 & 0.42 & 1.02 & 0.46 & 1.12 \\
\hline & error +1 - & $0.09 / 0.08$ & $0.08 / 0.07$ & $0.34 / 0.24$ & $0.29 / 0.17$ & $0.13 / 0.12$ & $0.15 / 0.11$ & $0.07 / 0.07$ \\
\hline & $p$-value & $<0.001$ & 0.038 & 0.12 & 0.02 & 0.94 & $<0.001$ & 0.44 \\
\hline \multirow[t]{3}{*}{ aP2 } & fold & 0.52 & 0.72 & 1.09 & 0.31 & 0.82 & 0.47 & 1.06 \\
\hline & error $+1-$ & $0.13 / 0.11$ & $0.05 / 0.05$ & $0.13 / 0.12$ & $0.25 / 0.14$ & $0.07 / 0.07$ & $0.15 / 0.11$ & $0.07 / 0.06$ \\
\hline & $p$-value & $<0.001$ & 0.005 & 0.26 & 0.007 & 0.04 & $<0.001$ & 0.45 \\
\hline \multirow[t]{3}{*}{ Acrp30 } & fold & 0.41 & 0.61 & 0.85 & 0.39 & 0.84 & 0.69 & 1.11 \\
\hline & error $+1-$ & $0.13 / 0.10$ & $0.12 / 0.10$ & $0.11 / 0.10$ & $0.39 / 0.19$ & $0.09 / 0.08$ & $0.08 / 0.07$ & $0.14 / 0.13$ \\
\hline & $p$-value & $<0.001$ & 0.002 & 0.06 & 0.02 & 0.79 & $<0.001$ & 0.11 \\
\hline \multirow[t]{3}{*}{ Glut4 } & fold & 0.25 & 0.39 & 1.33 & 0.37 & 0.86 & 0.42 & 1.04 \\
\hline & error +1 - & $0.10 / 0.07$ & $0.09 / 0.08$ & $0.21 / 0.18$ & $0.42 / 0.20$ & $0.17 / 0.15$ & $0.07 / 0.06$ & $0.20 / 0.17$ \\
\hline & $p$-value & $<0.001$ & $<0.001$ & 0.04 & 0.06 & 0.33 & $<0.001$ & 0.47 \\
\hline \multirow[t]{3}{*}{$\overline{L P L}$} & fold & 0.39 & 0.62 & 0.41 & 0.10 & 0.63 & 0.77 & 0.73 \\
\hline & error + / - & $0.17 / 0.12$ & $0.13 / 0.10$ & $0.35 / 0.19$ & $0.40 / 0.08$ & $0.28 / 0.19$ & $0.12 / 0.11$ & $0.59 / 0.33$ \\
\hline & $p$-value & $<0.001$ & 0.013 & 0.09 & 0.005 & 0.01 & 0.13 & 0.18 \\
\hline \multirow[t]{3}{*}{ FAS } & fold & 0.63 & 0.65 & 0.79 & 0.68 & 1.13 & 0.83 & 1.05 \\
\hline & error +1 - & $0.05 / 0.05$ & $0.05 / 0.05$ & $0.19 / 0.15$ & $0.06 / 0.06$ & $0.19 / 0.17$ & $0.07 / 0.07$ & $0.07 / 0.07$ \\
\hline & $p$-value & $<0.001$ & 0.02 & 0.14 & 0.04 & 0.37 & 0.08 & 0.37 \\
\hline \multirow[t]{3}{*}{$\overline{H S L}$} & fold & 0.58 & 0.75 & 0.91 & 0.49 & 1.04 & 0.69 & 1.05 \\
\hline & error +1 - & $0.11 / 0.09$ & $0.11 / 0.10$ & $0.06 / 0.06$ & $0.28 / 0.18$ & $0.10 / 0.09$ & $0.09 / 0.08$ & $0.07 / 0.07$ \\
\hline & $p$-value & $<0.001$ & 0.016 & 0.58 & 0.04 & 0.69 & $<0.001$ & 0.30 \\
\hline \multirow[t]{3}{*}{ СРТ-1 $\beta$} & fold & 0.21 & 0.34 & 1.11 & 0.59 & 0.81 & 0.44 & 0.84 \\
\hline & error $+1-$ & $0.09 / 0.06$ & $0.11 / 0.08$ & $0.28 / 0.22$ & $0.27 / 0.18$ & $0.10 / 0.09$ & $0.06 / 0.05$ & $0.09 / 0.08$ \\
\hline & $p$-value & $<0.001$ & $<0.001$ & 0.23 & 0.35 & 0.08 & $<0.001$ & 0.47 \\
\hline \multirow[t]{3}{*}{ ACC1 } & fold & 0.57 & 0.64 & 0.90 & 0.88 & 1.46 & 0.68 & 1.17 \\
\hline & error + / - & $0.14 / 0.11$ & $0.14 / 0.11$ & $0.30 / 0.23$ & $0.01 / 0.01$ & $0.34 / 0.27$ & $0.15 / 0.13$ & $0.19 / 0.17$ \\
\hline & $p$-value & 0.004 & 0.02 & 0.58 & 0.72 & 0.09 & 0.02 & 0.45 \\
\hline
\end{tabular}

With regard to the glucose transporter 4 gene (Glut4), which is instrumental for adipocyte glucose uptake, the investigated substances had an effect that was similar to that observed for ACC1 (Figure 2.5). In particular, DHA affected the gene expression of Glut4 in a dose-dependent manner. Conversely, resveratrol slightly increased mRNA levels of the investigated genes (Figure 2.5). This is consistent with the effects on cell morphology described above. 
Novel fluorescent method for LD quantification

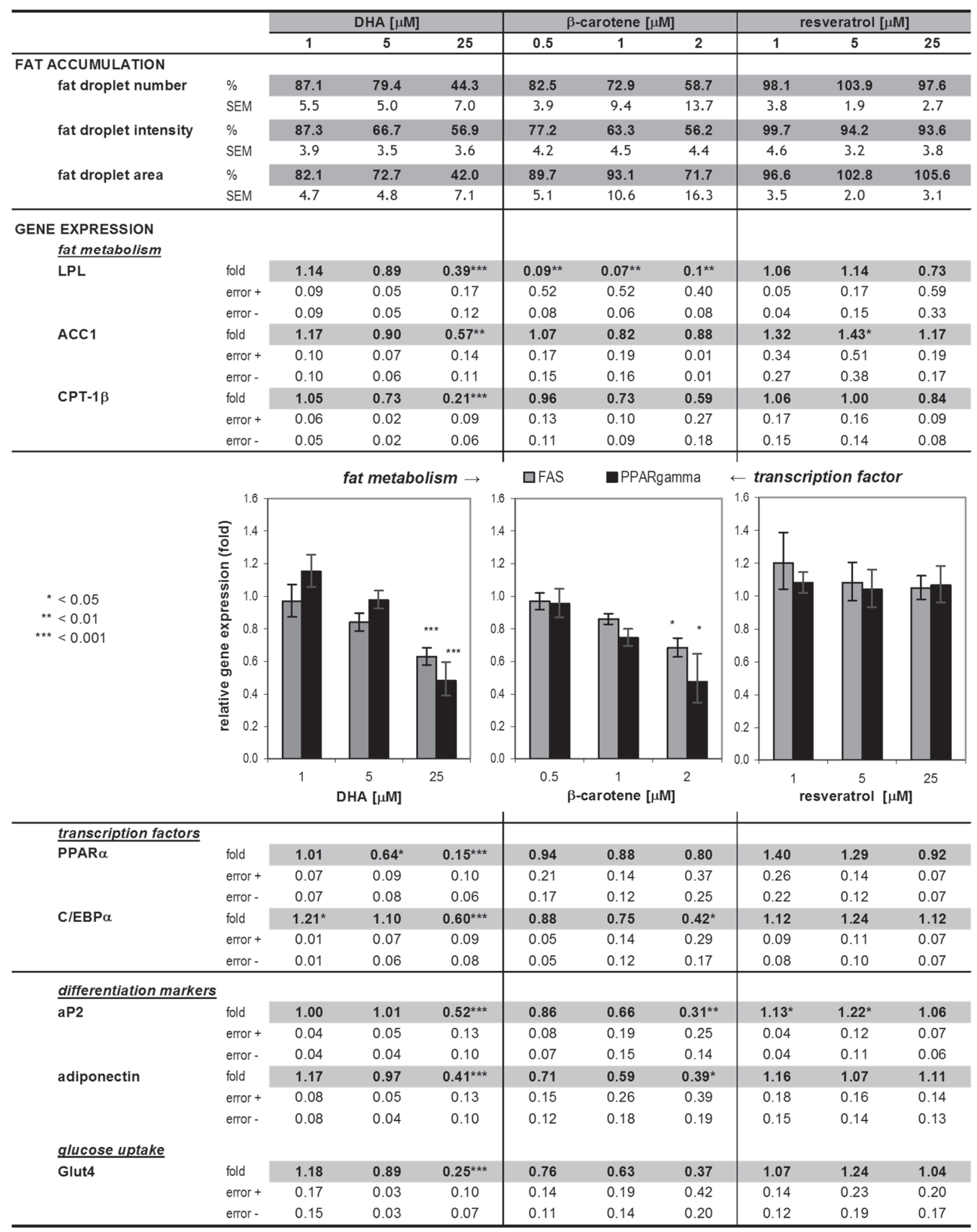

Figure 2.5: Summary of overall effects of the dietary ingredients DHA, $\beta$-carotene and resveratrol on lipid accumulation and on gene expression clusters

mRNA levels of enzymes involved in glucose and fat metabolism (Glut4, LPL, FAS, ACC1 and CPT-1 $\beta$ ) and of adipocyte differentiation markers (PPAR $\gamma, \mathrm{C} / \mathrm{EBP} \alpha, \mathrm{PPAR} \alpha$, aP2 and adiponectin) were determined in maturing $\mathrm{C} 3 \mathrm{H} 10 \mathrm{~T} 1 / 2$ cells. Depicted are the dose-dependent effects of DHA and $\beta$-carotene and the impact of resveratrol on lipid accumulation (after 7 days treatment, top of the table) and on gene expression (after 4 days treatment) relative to rosiglitazone control cells. Data are shown as $\%$ of 
positive control \pm SEM (fat accumulation parameters) and fold change \pm error (based on SEM, $n=6-15$ ) for gene expression levels, respectively. Exemplarily, the fold changes of the genes FAS and PPAR $\square$ are shown as illustration. Fat droplet number equates to Spot Count / Object; fat droplet intensity equates to Spot Avg Intensity and fat droplet area equates to Spot Total Area / Object. Student's t-test: treatment versus control $\left({ }^{*}\right) p<0.05,\left({ }^{* *}\right) p<0.01,\left({ }^{* *}\right) p<0.001$. The average coefficient of variation $(C V)$ for $d C T$ values was less than $5 \%$ for all analyzed genes at all concentration of the investigated compounds.

\section{Discussion}

The present study describes the ability of natural substances to modulate intracellular lipid accumulation during adipogenesis. A high-content method for detecting fat droplets in differentiating adipocytes is established. Lipid droplets are easily accessible for microscopic analysis, because the nuclei and the droplets can be well visualised in separate fluorescent channels. As a model system, we selected a murine pluripotent cell line, C3H10 T1/2, which could be readily differentiated into adipocytes by treating the cells with the PPAR $\gamma$ agonist rosiglitazone and insulin.

The SpotDetector ${ }^{\circledR}$ protocol from ThermoFisher was modified to identify fat droplets with a radius of $>15$ pixel (i.e. $>3.9 \mu \mathrm{m}$ ), therefore excluding small droplets that are formed in pre-adipocytes only exposed to DMSO and insulin. The adapted algorithm quantified the effects of $10 \mu \mathrm{M}$ rosiglitazone with high consistency, yielding Z-factors $>0.6$ for the nuclei-related parameters Spot Count and Spot Area and for the parameter Spot Average Intensity. Hence the Cellomics ${ }^{\circledR}$ assay is suitable for rapid screening of a large number of compounds [19] and is amendable for a high throughput screen if transferred to a 96-well-plate format. The consistent measurements achieved with the SpotDetector ${ }^{\circledR}$ BioApplication also enabled detection of moderate effects of less potent modulators such as bioactive components in food. This high-content analysis (HCA) approach is a greatly improved method (higher Z-factors, broader window and small CVs) compared with the commonly-used Oil Red O procedure, delivering fast, multi-parametric and objective data in a labour-reduced manner. Its use is comparable with the system presented by McDonough and coworkers [20], who applied the CyteSeer ${ }^{\circledR}$ image analysis software (Vala Sciences), together with a robotic microscopy instrument. However, the convenient use of commercially available cell culture plates, the easily applicable lipid stain (BODIPY ${ }^{\circledR}$ 493/503) and rapid field-based analysis of images with the provided BioApplication from ThermoFisher are advantageous for the fast quantification of fat droplets in adipocytes.

A large number of studies suggest that certain plant extracts, their constituent phytochemicals [8, 21] and the fatty acid composition of fats [22], influence the metabolism in adipocytes [23]. Based 
on this information we tested several bioactive substances present in our daily diet with the established HCA assay.

The $\omega-3$ PUFAs EPA and DHA, which are known to reduce lipid droplet size in differentiating 3T3-L1 pre-adipocytes $[9,24]$ and to decrease adipose growth in rodents [25-28], were tested as reference compounds in our assay. $\omega-3$ PUFAs markedly reduced the number of fat droplets and their average intensity in a dose-dependent manner whereas size of the lipid droplets was not decreased (data not shown). By comparison, Madsen et al. [9] used a mix of dexamethasone, 3isobutyl-1-methylxanthine (IBMX) and insulin for the differentiation of 3T3-L1 cells and compared whether different PUFAs enhanced adipocyte differentiation to a similar extent as the PPAR $\gamma$ agonist rosiglitazone. DHA and EPA were reported to be less potent than rosiglitazone. Because we used only rosiglitazone and insulin for differentiation, one could expect that the concomitant treatment with DHA or EPA would further stimulate the TG accretion. Interestingly in maturing $\mathrm{C} 3 \mathrm{H} 10 \mathrm{~T} 1 / 2$ cells we observed the opposite effect. At which stages of the adipocyte life cycle (determination, clonal expansion, maturation, lipolysis or apoptosis) [2] the $\omega$-3 PUFAs affect the reduction of lipid droplets in $\mathrm{C} 3 \mathrm{H} 10$ T1/2 could not be deduced from the ArrayScan ${ }^{\circledR}$ results. The elevated LDH levels after 7 days' treatment might indicate that DHA induced apoptosis during clonal expansion as described for 3T3-L1 cells [29]. Thus, the potent inhibition of lipid accumulation is not only due to decreased adipogenesis but might also reflect a proapoptotic effect of DHA.

In addition, we showed that EPA and DHA reduced expression levels of adipogenic and lipogenic genes as described by Raclot et al. [30] and Shillabeer et al. [31]. C/EBP $\alpha$ and PPAR $\gamma$ are the 'master' regulators of adipogenesis [32]. PPAR $\gamma$ regulates the anabolic arm of lipid metabolism [33], whereas PPAR $\alpha[5,34]$ and PPAR $\beta / \delta$ are effective as catabolic modulators of the energy balance [35]. PPAR $\gamma$ and PPAR $\alpha$ are induced in the early and late phases of the differentiation process, respectively [33, 36], and act as sensors for fatty acids and their derivatives [37, 38]. Our findings that $\mathrm{C} 3 \mathrm{H} 10 \mathrm{~T} 1 / 2$ adipocytes, differentiated in the presence of DHA or EPA, show only a weak C/EBP $\alpha$ and PPAR $\gamma$ expression, are in line with the results of Worgall et al:: PUFAs downregulated the expression of sterol regulatory element-binding proteins (SREBPs), which play a major role in adipocyte differentiation [39], regulate PPAR $\gamma$, and thereby suppress lipogenesis [40]. Moreover, Okuno et al. demonstrated that $\omega-3$ PUFAs also down-regulated genes of the late phase of adipocyte differentiation, such as PPAR $\alpha$ and aP2 in rat visceral adipose tissue [27].

We hypothesise that the reduced expression levels of these two genes and other enzymes and transporters important for lipid and glucose metabolism, such as LPL, FAS, CPT-1 $\beta$ and Glut4, 
are a consequence of DHA inhibiting the two master regulators PPAR $\gamma$ and $\mathrm{C} / \mathrm{EBP} \alpha$ and thus suppressing adipogenesis and concomitant lipogenesis in C3H10 T1/2 cells. Collectively, our data are both consistent with and extend earlier studies performed in 3T3-L1 cells [24, 29, 41].

Furthermore, a large body of studies concerning $\beta$-carotene and lycopene, EGCG and resveratrol demonstrates that the effects of natural compounds on adipocyte differentiation are as varying as their structure [23]. EGCG, the major catechin in green tea, is known to stimulate apoptosis, inhibit adipogenesis and intracellular TG accretion in 3T3-L1 adipocytes [42, 43]. Human AML-1 cells undergo apoptosis after treatment with EGCG, although the conversion from pre-adipocytes to adipocytes is not affected [44]. Moreover, EGCG had no lipolytic influence on mature C3H10 T1/2 adipocytes [45]. Our results show that EGCG only moderately inhibited the average intensity of the fat droplets in $\mathrm{C} 3 \mathrm{H} 10 \mathrm{~T} 1 / 2$ cells and it had no significant impact on any gene expression levels, although Furuyashiki et al. [43] reported that in 3T3-L1 cells PPAR $\gamma$ and $\mathrm{C} / \mathrm{EBP} \alpha$ were down-regulated by tea catechins, like EGCG. These also showed an enhancing effect on the expression and secretion of adiponectin in 3T3-L1 adipocytes [46]. However, in maturing $\mathrm{C} 3 \mathrm{H} 10 \mathrm{~T} 1 / 2$ adipocytes the mRNA level of adiponectin was not affected by EGCG. Investigations on the protein level and of the cell supernatants are required to further determine the effects of dietary constituents on secreted adipokines.

Resveratrol has been tested on adipocytes [47] and was found to be apoptotic [48], anti-adipogenic $[48,49]$ and anti-lipogenic [21]. In this study resveratrol showed no inhibitory effect, or even induced a moderate stimulation of lipid accumulation in differentiating $\mathrm{C} 3 \mathrm{H} 10 \mathrm{~T} 1 / 2$ cells. This is consistent with the observed up-regulation of aP2 mRNA at low resveratrol concentrations, compared to rosiglitazone control. In contrast, Rayalam et al. [48] reported suppression of adipocyte-specific genes such as PPAR $\gamma, \mathrm{C} / \mathrm{EBP} \alpha, \mathrm{FAS}, \mathrm{HSL}$ and LPL and a strong inhibition of lipid accumulation ( $40 \%$ by $25 \mu \mathrm{M}$ resveratrol) in maturing 3T3-L1 adipocytes. These effects were accounted for by decreased cell viability.

Reduced cell number and increased LDH levels are indicative of apoptosis and reduced cell viability [48]. Consequently, a diminished number of fat cells during differentiation enable less fat accumulation. It can be assumed that $\beta$-carotene induced apoptosis at $2 \mu \mathrm{M}$ similar to EGCG at $25 \mu \mathrm{M}$ (data not shown, [42]). At this physiologically-relevant concentration $\beta$-carotene efficiently reduced fat droplet number, size, and intensity. Furthermore $\beta$-carotene treatment led to a downregulation of the dominant regulators of adipogenesis, $\operatorname{PPAR} \gamma$ and $\mathrm{C} / \mathrm{EBP} \alpha$, and a significant reduction of LPL, HSL, FAS, aP2 and adiponectin. The effects of $\beta$-carotene and its biologically active form vitamin A (retinol / retinal / retinoic acid) on WAT are well-studied (for review see 
[50]). Kawada and co-workers [51] described that fat-soluble vitamins, including provitamin $\beta$ carotene, strongly inhibited adipose conversion of 3T3-L1 cells. They [52] suggested that carotenoids and retinoids inhibit adipocyte differentiation through retinoic acid receptor upregulation and the decrease of PPAR $\gamma 2$. The strong suppression of LPL, one of the early markers of adipocyte differentiation [53], is remarkable compared to all the other dose-dependent effects of $\beta$-carotene. Insulin is known to induce LPL synthesis in adipocytes and stimulate LPL, ACC and Glut4 proteins. Plausibly, $\beta$-carotene - even in low concentrations - interferes with insulin actions, although recently it was shown by Kameji et al. [54] that high concentrations of $\beta$-carotene elevated LPL and PPAR $\gamma$ levels and insulin functions were supported. Lycopene, as a fat-soluble molecule is also stored in adipose tissue, in particular in different cellular compartments of the adipocytes [55]. Our results illustrate that lycopene decreased the accumulation of TGs to a far lesser extent than $\beta$-carotene and it also showed no effect on the different adipogenic and lipogenic genes. Only Glut4, which participates in insulin-dependent glucose uptake into adipocytes [56] and is thus indirectly involved in fatty acid synthesis, was significantly induced. Therefore, the small effect it has must be mediated via other mechanisms than PPAR $\gamma$ and C/EBP $\alpha$ suppression. Hydroxytyrosol (HT) is the major polyphenol in extra-virgin olive oil and its potent antioxidant properties are thought to be partly responsible for the Mediterranean diet effect $[57,58]$. In our study, HT showed effects that were comparable to those of EPA and $\beta$-carotene on adipocyte lipid content and gene expression. HT significantly inhibited all tested genes except LPL.

In summary, dietary bio-actives such as $\omega-3$ PUFAs, $\beta$-carotene and HT, which seem to have the same inhibitory effect on lipid accumulation during adipocyte differentiation, display different impacts on the expression of relevant genes. Therefore, the influence of substances on the phosphorylation status of corresponding enzymes, e.g. LPL and HSL, should also be considered. The conflicting data reported in different studies might be partly explained by the use of different cell models from different species. Adipose tissue biology is diversely regulated among different species, thus the same might be applicable for different cell lines. Contradictory effects of substances in diverse cell systems could be explained due to the application of cAMP elevating agents, the hormonal status (e.g. dexamethasone and insulin) present during adipocyte differentiation [9] and the stage of the adipocyte life cycle at which they are applied [23]. 


\section{Conclusion}

Obesity is characterised by increased adipose tissue mass and is associated with high health risks. The expansion of WAT is conditional upon its ability to increase the number of adipocytes and their volume (triglyceride content in fat droplets). Identifying compounds that suppress the formation of new adipocytes and the accretion of fat at different stages of the adipocyte life cycle is of major interest for preventive measures and therapeutic applications. Our data show that this newly developed HCA assay is a valuable tool to identify and quantify changes in fat accumulation during adipogenesis and lipogenesis and thus for the identification of such substances. This study demonstrates that treatment of murine $\mathrm{C} 3 \mathrm{H} 10 \mathrm{~T} 1 / 2$ pre-adipocytes with naturally-occurring components can reduce the amount of accumulated fat. Furthermore, the observed morphological changes are in line with the alterations in expression levels of adipogenic and lipogenic genes.

Murine cell lines might give indications how food ingredients influence the formation of fat droplets in tissues. This needs however, to be further corroborated by experiments with human adipocytes. Ultimately, studies with obese humans would be of great value, as obesity is not only caused by high fat consumption but also triggered by high intakes of refined carbohydrates [9]. Other research groups approach this challenge and discovered both differences and analogies in human and rodent adipogenesis [59]. For example, Söhle et al. describes that a white tea extract rich in polyphenols inhibited the adipogenesis in human subcutaneous pre-adipocytes [60].

Because several natural compounds act in different ways on the elaborate biological pathways of adipose tissue formation, it would be advantageous to treat adipocytes with a combination of dietary bio-actives. Hence, such an approach might exceed the favourable effects of each individual compound and lead to additive or synergistic effects on multiple levels of adipocyte differentiation. This strategy might also apply for improving the appearance of dimpled skin caused by overfilled subcutaneous adipocytes mostly observed in obese people.

\section{Acknowledgements}

We would like to thank Christine Töpfer for helping to perform RNA extractions, Nicole Seifert for cell culture support, Ann Fowler for assistance with the ArrayScan ${ }^{\circledR}$ reader and protocol establishment and Annis Mechan for critical review of the manuscript. 


\section{References}

1. Formiguera X, Canton A: Obesity: epidemiology and clinical aspects. 2004, 18:1125-1146.

2. Gregoire FM: Adipocyte differentiation: from fibroblast to endocrine cell. Exp Biol Med (Maywood) 2001, 226:997-1002.

3. Rosen ED, Walkey CJ, Puigserver P, Spiegelman BM: Transcriptional regulation of adipogenesis. Genes Dev 2000, 14:1293-1307.

4. Spalding KL, Arner E, Westermark PO, Bernard S, Buchholz BA, Bergmann O, Blomqvist L, Hoffstedt J, Naslund E, Britton T, Concha H, Hassan M, Ryden M, Frisen J, Arner P: Dynamics of fat cell turnover in humans. Nature 2008, 453:783-787.

5. Tontonoz P, Hu E, Spiegelman BM: Stimulation of adipogenesis in fibroblasts by PPAR gamma 2, a lipid-activated transcription factor. Cell 1994, 79:1147-1156.

6. Freytag SO, Paielli DL, Gilbert JD: Ectopic expression of the CCAAT/enhancer-binding protein alpha promotes the adipogenic program in a variety of mouse fibroblastic cells. Genes Dev 1994, 8:1654-1663.

7. Shimomura I, Hammer RE, Richardson JA, Ikemoto S, Bashmakov Y, Goldstein JL, Brown MS: Insulin resistance and diabetes mellitus in transgenic mice expressing nuclear SREBP-1c in adipose tissue: model for congenital generalized lipodystrophy. Genes Dev 1998, 12:3182-3194.

8. Hsu CL, Yen GC: Phenolic compounds: evidence for inhibitory effects against obesity and their underlying molecular signaling mechanisms. Mol Nutr Food Res 2008, 52:53-61.

9. Madsen L, Petersen RK, Kristiansen K: Regulation of adipocyte differentiation and function by polyunsaturated fatty acids. Biochim Biophys Acta 2005, 1740:266-286.

10. Green H, Meuth M: An established pre-adipose cell line and its differentiation in culture. Cell 1974, 3:127-133.

11. Todaro GJ, Green H: Quantitative studies of the growth of mouse embryo cells in culture and their development into established lines. J Cell Biol 1963, 17:299-313.

12. Reznikoff CA, Brankow DW, Heidelberger C: Establishment and characterization of a cloned line of C3H mouse embryo cells sensitive to postconfluence inhibition of division. Cancer Res 1973, 33:3231-3238.

13. Auwerx J, Leroy P, Schoonjans K: Lipoprotein lipase: recent contributions from molecular biology. Crit Rev Clin Lab Sci 1992, 29:243-268.

14. Matarese V, Bernlohr DA: Purification of murine adipocyte lipid-binding protein. Characterization as a fatty acid- and retinoic acid-binding protein. J Biol Chem 1988, 263:14544-14551.

15. Kim JB, Sarraf P, Wright M, Yao KM, Mueller E, Solanes G, Lowell BB, Spiegelman BM: Nutritional and insulin regulation of fatty acid synthetase and leptin gene expression through ADD1/SREBP1. J Clin Invest 1998, 101:1-9.

16. Sztalryd C, Komaromy MC, Kraemer FB: Overexpression of hormone-sensitive lipase prevents triglyceride accumulation in adipocytes. J Clin Invest 1995, 95:2652-2661.

17. Ramirez-Zacarias JL, Castro-Munozledo F, Kuri-Harcuch W: Quantitation of adipose conversion and triglycerides by staining intracytoplasmic lipids with Oil red O. Histochemistry 1992, 97:493-497.

18. Kast-Woelbern HR, Dana SL, Cesario RM, Sun L, de Grandpre LY, Brooks ME, Osburn DL, ReifelMiller A, Klausing K, Leibowitz MD: Rosiglitazone induction of Insig-1 in white adipose tissue reveals a novel interplay of peroxisome proliferator-activated receptor gamma and sterol regulatory element-binding protein in the regulation of adipogenesis. J Biol Chem 2004, 279:23908-23915.

19. Zhang JH, Chung TD, Oldenburg KR: A Simple Statistical Parameter for Use in Evaluation and Validation of High Throughput Screening Assays. J Biomol Screen 1999, 4:67-73.

20. McDonough PM, Agustin RM, Ingermanson RS, Loy PA, Buehrer BM, Nicoll JB, Prigozhina NL, Mikic I, Price JH: Quantification of lipid droplets and associated proteins in cellular models of obesity via high-content/high-throughput microscopy and automated image analysis. Assay Drug Dev Technol 2009, 7:440-460.

21. Hsu CL, Yen GC: Effects of flavonoids and phenolic acids on the inhibition of adipogenesis in 3T3L1 adipocytes. J Agric Food Chem 2007, 55:8404-8410.

22. Nakatani T, Kim HJ, Kaburagi Y, Yasuda K, Ezaki O: A low fish oil inhibits SREBP-1 proteolytic cascade, while a high-fish-oil feeding decreases SREBP-1 mRNA in mice liver: relationship to antiobesity. J Lipid Res 2003, 44:369-379. 
23. Rayalam S, Della-Fera MA, Baile CA: Phytochemicals and regulation of the adipocyte life cycle. J Nutr Biochem 2008, 19:717-726.

24. Manickam E, Sinclair AJ, Cameron-Smith D: Suppressive actions of eicosapentaenoic acid on lipid droplet formation in 3T3-L1 adipocytes. Lipids Health Dis 2010, 9:57.

25. Shimomura Y, Tamura T, Suzuki M: Less body fat accumulation in rats fed a safflower oil diet than in rats fed a beef tallow diet. J Nutr 1990, 120:1291-1296.

26. Wang H, Storlien LH, Huang XF: Effects of dietary fat types on body fatness, leptin, and ARC leptin receptor, NPY, and AgRP mRNA expression. Am J Physiol Endocrinol Metab 2002, 282:E1352-9.

27. Okuno M, Kajiwara K, Imai S, Kobayashi T, Honma N, Maki T, Suruga K, Goda T, Takase S, Muto Y, Moriwaki H: Perilla oil prevents the excessive growth of visceral adipose tissue in rats by downregulating adipocyte differentiation. J Nutr 1997, 127:1752-1757.

28. Pellizzon M, Buison A, Ordiz F,Jr, Santa Ana L, Jen KL: Effects of dietary fatty acids and exercise on body-weight regulation and metabolism in rats. Obes Res 2002, 10:947-955.

29. Kim HK, Della-Fera M, Lin J, Baile CA: Docosahexaenoic acid inhibits adipocyte differentiation and induces apoptosis in 3T3-L1 preadipocytes. J Nutr 2006, 136:2965-2969.

30. Raclot T, Groscolas R, Langin D, Ferre P: Site-specific regulation of gene expression by n-3 polyunsaturated fatty acids in rat white adipose tissues. J Lipid Res 1997, 38:1963-1972.

31. Shillabeer G, Hornford J, Forden JM, Wong NC, Lau DC: Hepatic and adipose tissue lipogenic enzyme mRNA levels are suppressed by high fat diets in the rat. J Lipid Res 1990, 31:623-631.

32. Farmer SR: Transcriptional control of adipocyte formation. Cell Metab 2006, 4:263-273.

33. Chawla A, Schwarz EJ, Dimaculangan DD, Lazar MA: Peroxisome proliferator-activated receptor (PPAR) gamma: adipose-predominant expression and induction early in adipocyte differentiation. Endocrinology 1994, 135:798-800.

34. Pyper SR, Viswakarma N, Yu S, Reddy JK: PPARalpha: energy combustion, hypolipidemia, inflammation and cancer. Nucl Recept Signal 2010, 8:e002.

35. Wang YX, Lee CH, Tiep S, Yu RT, Ham J, Kang H, Evans RM: Peroxisome-proliferator-activated receptor delta activates fat metabolism to prevent obesity. Cell 2003, 113:159-170.

36. Chawla A, Lazar MA: Peroxisome proliferator and retinoid signaling pathways co-regulate preadipocyte phenotype and survival. Proc Natl Acad Sci U S A 1994, 91:1786-1790.

37. Chawla A, Repa JJ, Evans RM, Mangelsdorf DJ: Nuclear receptors and lipid physiology: opening the X-files. Science 2001, 294:1866-1870.

38. Krey G, Braissant O, L'Horset F, Kalkhoven E, Perroud M, Parker MG, Wahli W: Fatty acids, eicosanoids, and hypolipidemic agents identified as ligands of peroxisome proliferator-activated receptors by coactivator-dependent receptor ligand assay. Mol Endocrinol 1997, 11:779-791.

39. Kim JB, Spiegelman BM: ADD1/SREBP1 promotes adipocyte differentiation and gene expression linked to fatty acid metabolism. Genes Dev 1996, 10:1096-1107.

40. Worgall TS, Sturley SL, Seo T, Osborne TF, Deckelbaum RJ: Polyunsaturated fatty acids decrease expression of promoters with sterol regulatory elements by decreasing levels of mature sterol regulatory element-binding protein. J Biol Chem 1998, 273:25537-25540.

41. Li HX, Yang GS: [Effects of docosahexaenoic acid on rat adipocytes proliferation and differentiation]. Sheng Wu Gong Cheng Xue Bao 2005, 21:840-843.

42. Lin J, Della-Fera MA, Baile CA: Green tea polyphenol epigallocatechin gallate inhibits adipogenesis and induces apoptosis in 3T3-L1 adipocytes. Obes Res 2005, 13:982-990.

43. Furuyashiki T, Nagayasu H, Aoki Y, Bessho H, Hashimoto T, Kanazawa K, Ashida H: Tea catechin suppresses adipocyte differentiation accompanied by down-regulation of PPARgamma2 and C/EBPalpha in 3T3-L1 cells. Biosci Biotechnol Biochem 2004, 68:2353-2359.

44. Morikawa K, Ikeda C, Nonaka M, Pei S, Mochizuki M, Mori A, Yamada S: Epigallocatechin gallateinduced apoptosis does not affect adipocyte conversion of preadipocytes. Cell Biol Int 2007, 31:13791387.

45. Wolfram S, Wang Y, Thielecke F: Anti-obesity effects of green tea: from bedside to bench. Mol Nutr Food Res 2006, 50:176-187.

46. Cho SY, Park PJ, Shin HJ, Kim YK, Shin DW, Shin ES, Lee HH, Lee BG, Baik JH, Lee TR: (-)Catechin suppresses expression of Kruppel-like factor 7 and increases expression and secretion of adiponectin protein in 3T3-L1 cells. Am J Physiol Endocrinol Metab 2007, 292:E1166-72. 
47. Picard F, Kurtev M, Chung N, Topark-Ngarm A, Senawong T, Oliveira MD, Leid M, McBurney MW, Guarente L: Sirt1 promotes fat mobilization in white adipocytes by repressing PPAR-gamma. Nature 2004, 429:771-776.

48. Rayalam S, Yang JY, Ambati S, Della-Fera MA, Baile CA: Resveratrol induces apoptosis and inhibits adipogenesis in 3T3-L1 adipocytes. Phytother Res 2008, 22:1367-1371.

49. Pang WJ, Sun SD, Bai L, Yang YJ, Yang GS: [Effects of resveratrol on pig primary preadipocytes proliferation, differentiation and transcription expression of Sirt1 gene]. Sheng Wu Gong Cheng Xue Bao 2006, 22:850-855.

50. Tourniaire F, Gouranton E, von Lintig J, Keijer J, Luisa Bonet M, Amengual J, Lietz G, Landrier JF: beta-Carotene conversion products and their effects on adipose tissue. Genes Nutr 2009, 4:179-187.

51. Kawada T, Aoki N, Kamei Y, Maeshige K, Nishiu S, Sugimoto E: Comparative investigation of vitamins and their analogues on terminal differentiation, from preadipocytes to adipocytes, of 3T3L1 cells. Comp Biochem Physiol A Comp Physiol 1990, 96:323-326.

52. Kawada T, Kamei Y, Fujita A, Hida Y, Takahashi N, Sugimoto E, Fushiki T: Carotenoids and retinoids as suppressors on adipocyte differentiation via nuclear receptors. Biofactors 2000, 13:103109.

53. Ailhaud G, Grimaldi P, Negrel R: Cellular and molecular aspects of adipose tissue development. Annu Rev Nutr 1992, 12:207-233.

54. Kameji H, Mochizuki K, Miyoshi N, Goda T: beta-Carotene accumulation in 3T3-L1 adipocytes inhibits the elevation of reactive oxygen species and the suppression of genes related to insulin sensitivity induced by tumor necrosis factor-alpha. Nutrition 2010,

55. Gouranton E, Yazidi CE, Cardinault N, Amiot MJ, Borel P, Landrier JF: Purified low-density lipoprotein and bovine serum albumin efficiency to internalise lycopene into adipocytes. Food Chem Toxicol 2008, 46:3832-3836.

56. Bryant NJ, Govers R, James DE: Regulated transport of the glucose transporter GLUT4. Nat Rev Mol Cell Biol 2002, 3:267-277.

57. Bendini A, Cerretani L, Carrasco-Pancorbo A, Gomez-Caravaca AM, Segura-Carretero A, Fernandez-Gutierrez A, Lercker G: Phenolic molecules in virgin olive oils: a survey of their sensory properties, health effects, antioxidant activity and analytical methods. An overview of the last decade. Molecules 2007, 12:1679-1719.

58. Fito M, de la Torre R, Covas MI: Olive oil and oxidative stress. Mol Nutr Food Res 2007, 51:12151224.

59. Entenmann G, Hauner H: Relationship between replication and differentiation in cultured human adipocyte precursor cells. Am J Physiol 1996, 270:C1011-6.

60. Söhle J, Knott A, Holtzmann U, Siegner R, Gronniger E, Schepky A, Gallinat S, Wenck H, Stab F, Winnefeld M: White Tea extract induces lipolytic activity and inhibits adipogenesis in human subcutaneous (pre)-adipocytes. 2009, 6:20. 


\section{CHAPTER 3}

Combinations of bio-active dietary constituents affect human white adipocyte function in-vitro

Ines Warnke, Johan W.E. Jocken, Rotraut Schoop, Christine Toepfer, Regina Goralczyk, Joseph Schwager Published in "Nutrition \& Metabolism" (2016) 13:84 


\section{Abstract}

Background: Specific bio-active dietary compounds modulate numerous metabolic processes in adipose tissue (AT), including pre-adipocyte proliferation and differentiation. AT dysfunction, rather than an increased fat mass per se, is strongly associated with the development of insulin resistance and is characterized by impaired adipogenesis, hypertrophic adipocytes, inflammation, and impairments in substrate metabolism. A better understanding of mechanisms underlying AT dysfunction may provide new strategies for the treatment of obesity-associated metabolic diseases. Here we evaluated the role of (all-E)lycopene (Lyc), eicosapentaenoic acid (EPA) or trans-resveratrol (Res) and combinations thereof on human white adipocyte function.

Methods: In-vitro differentiating human pre-adipocytes were treated with EPA, Lyc and Res or their combinations for 14 days. The effects on intracellular lipid droplet (LD) accumulation, secreted anti- and pro-inflammatory cyto-/adipokines (e.g. adiponectin, IL-6, IL-8/CXCL-8 and MCP-1/CCL2) and on gene expression of markers of adipocyte differentiation and substrate metabolism (e.g., PPAR-gamma, C/EBPalpha, GLUT-4, FAS, ATGL, HSL, and PLIN-1) were measured by fluorescent microscopy (Cellomics ${ }^{\mathrm{TM}}$ ), multi-parametric LiquiChip ${ }^{\circledR}$ technology and quantitative RT-PCR, respectively.

Results: Treatment of differentiating adipocytes for 14 days with the combination of Lyc/Res and EPA/Res resulted in significantly inhibited LD formation ( $\sim-25 \%$ and $-20 \%$, respectively) compared to the effects of the single compounds. These morphological changes were accompanied by increased mRNA levels of the adipogenic marker PPAR-gamma and the lipase ATGL and by decreased expression levels of lipogenic markers (LPL, FAS, GLUT-4) and the LD-covering protein PLIN-1. In addition, a blunted adipocyte secretion of pro-inflammatory cytokines (IL-6 and MCP-1) and adiponectin was observed following treatment with these compounds.

Conclusion: The combination of the dietary bio-actives Lyc and EPA with Res might influence adipocyte function by affecting the balance between adipogenic, lipogenic and lipolytic gene expression, resulting in a reduced LD storage and a less inflammatory secretion profile. Taken together, our results indicate that combinations of dietary compounds may be beneficial for the prevention and treatment of metabolic disorders via effects on human white adipocyte function. 


\section{Background}

Over the last decade research focusing on adipose tissue (AT) biology and function enormously advanced due to the sustained increase in obesity prevalence [1]. AT dysfunction, characterized by impaired adipogenesis, hypertrophic adipocytes, inflammation, and impairments in lipid and glucose metabolism, rather than an increased body fat mass per se, is strongly associated with the development of insulin resistance [2]. A better understanding of mechanisms causing or maintaining AT dysfunction may provide novel and improved strategies for the treatment of obesity-associated metabolic diseases.

The major role of AT is storage and release of fatty acids (FAs) depending on energy intake and expenditure. FAs are stored in the form of triacylglycerides (TAGs) in intracellular lipid droplets (LDs) and released by lipolysis, the hydrolysis of TAGs into free FAs and glycerol via the action of intracellular lipases (including hormone sensitive lipase (HSL) and adipose triglyceride lipase (ATGL)). This storage and removal capacity (lipid turn-over) of AT is regulated by a tight alignment between adipogenic differentiation, lipogenesis and lipolysis [3, 4], which has been shown to be impaired in obesity and may modulate whole-body insulin sensitivity $[5,6]$. The differentiation of pre-adipocytes into mature adipocytes is controlled by a complex transcriptional cascade involving peroxisome proliferator-activated receptor gamma (PPAR-gamma) and CCAAT/enhancer binding protein alpha (C/EBP-alpha) (reviewed in [7]). Furthermore, lipidstoring adipocytes are enclosed by adipose-derived stromal cells including pre-adipocytes, endothelial and hematopoietic cells, and immune cells (e.g. macrophages [8]). Hence, AT is not only an energy storage tissue but also an active endocrine organ, producing and secreting an abundance of specific mediators (for review see [9]). Pro-inflammatory cytokines and chemokines are increasingly secreted by AT cells of obese individuals [10], resulting in a state of 'low-grade inflammation' [11], which affects local adipose metabolism, systemic inflammation, and insulin sensitivity.

Overall dietary quality and specifically diets high in bio-active constituents may have beneficial clinical effects on metabolic processes, by altering AT function. A number of natural compounds, such as plant-derived polyphenols, carotenoids and polyunsaturated fatty acids (PUFAs), or their metabolic derivatives have been tested for their impact on adipocyte differentiation and metabolism in several in-vitro and in-vivo murine models [12-14]. However, human data are scarce and mainly the action of individual compounds have been tested $[15,16]$. Several studies using pre-adipocytes, mostly from murine and less frequent from human origin, have demonstrated that the polyphenol Res and the n-3 PUFA EPA are potent modulators of adipocyte function (for review 
see [17] and [18], respectively). However, distinct biological activities of lycopene or its metabolites in human adipocyte function remain to be elucidated. Here, we investigated the effects of individual and combinations of bio-active dietary constituents including (all-E)-lycopene (Lyc), eicosapentaenoic acid (EPA) and trans-resveratrol (Res) on lipid accumulation, adipogenic, lipogenic and lipolytic gene expression and cyto-/adipokine secretion in in-vitro differentiating (14 days) primary human white adipocytes.

\section{Material and Methods}

\section{Cell culture}

All cell culture reagents were obtained from Life Technologies (Karlsbad, CA, US). Unless otherwise stated, chemicals were purchased from Sigma-Aldrich (St. Louis, MO, US). Individual subcutaneous primary human pre-adipocytes (HPAd 1375 and 1377) were obtained from Cell Application, Inc. (San Diego, CA, US) whereas a multi-donor vial, termed super lot (SL0035), was purchased from Zen-Bio, Inc. (North Carolina, US). Available donor characteristics are indicated in Supplemental Table S3.1. Pre-adipocytes were maintained in growth medium (GM): DMEM/Ham's F-12 (1:1, v/v) complemented with 10\% FCS, 1\% pen/strep (v/v), 1\% HEPES pH $7.4,0.2 \%$ amphotericin $\mathrm{B}$, and $2.5 \mathrm{ng} / \mathrm{ml}$ recombinant basic fibroblast growth factor (bFGF). GM was changed every 2-3 days. Cells were passaged when reaching $\sim 80 \%$ confluence and used for experiments between passage 3 and 7. For experiments, 6000 cells $/ \mathrm{cm}^{2}$ were incubated $\left(37^{\circ} \mathrm{C}\right.$, 5$8 \% \mathrm{CO}_{2}$, relative humidity of $85 \%$ ) on collagen-I-coated 24 -well plates in GM for $\sim 5$ days. For adipogenesis confluent HPAd were cultured for 14 days in differentiation medium (DM): DMEM/Ham's F-12 supplemented with 5\% FCS, 1\% pen/strep, 1.5\% HEPES pH 7.4, 0.2\% amphotericin $\mathrm{B}, 17 \mu \mathrm{M}$ calcium-pantothenate, $33 \mu \mathrm{M}$ biotin, $0.5 \mu \mathrm{M}$ recombinant human insulin and $100 \mu \mathrm{M}$ rosiglitazone. After 3 days of differentiation $0.5 \mu \mathrm{M}$ dexamethasone and $250 \mu \mathrm{M}$ isobutylmethylxanthine (IBMX) were omitted from the medium.

For the non-differentiation control (CTRL) and differentiation control (Diff CTRL) pre-adipocytes were cultured in medium containing only vehicle (i.e. dimethylsulfoxide (DMSO), tetrahydrofurane (THF)) and insulin or in complete DM, respectively. For treatment purposes DM was supplemented with different doses $(0.5-25 \mu \mathrm{M})$ of the test compounds EPA, Res and Lyc (DSM Nutritional Products Ltd. Basel, Switzerland)) for the total 14-day differentiation period (see Supplemental Figure S3.1). Lyc was dissolved in THF and all other substances in DMSO. To investigate possible amplifying effects, Res $(1$ and $25 \mu \mathrm{M})$ was also combined with Lyc $(0.5$ and 
$2 \mu \mathrm{M})$ or EPA (1 and $25 \mu \mathrm{M})$, respectively. Treatments were performed in triplicate. Final vehicle concentrations were adjusted to $0.2 \%$ DMSO and $0.1 \%$ THF in all cultures. Media with or without bio-active compounds were renewed every 3 to 5 days.

\section{Lipid droplet quantification (ArrayScan)}

Cellular LDs were quantified adapting the Cellomics ${ }^{\mathrm{TM}}$ assay described previously [19] applying the Thermo Scientific ${ }^{\mathrm{TM}}$ ArrayScan ${ }^{\mathrm{TM}}$ VTI High Content Reader (Thermo Fisher Scientific, Waltham, MA, US). Briefly, differentiated adipocytes (day 14) were fixed, stained with the fluorescent dyes Hoechst 33342 (nuclei) and BODIPY ${ }^{\circledR}$ 493/503 (LDs; Life Technologies) followed by quantification of accumulated LDs with the SpotDetector ${ }^{\circledR}$ V2 algorithm. Adjusted protocol parameters are listed in Supplemental Table S3.2. For analysis 100 fields per well were scanned and data of each channel were reported on a "per field" basis. The nuclei related features Spot Count (=LD-number), Spot Total Area (= LD-area) and Spot Total Intensity (=LDintensity), describing the differentiation status of treated adipocytes, were calculated as percent of Diff CTRL per plate.

\section{Gene expression analysis (TaqMan ${ }^{T M}$ )}

Total RNA was isolated from cells at day 8 of treatment (RNeasy ${ }^{\circledR} 96$ Kits; Qiagen, Hilden, Germany). Primers and probes were designed using the Primer Express software (Applied Biosystems, Foster City, CA, US) and synthesised by Sigma Genosys (St. Louis, MO, US) (Supplemental Table S3.3). Quantitative TaqMan ${ }^{\text {TM }}$ RT-PCR was performed on first strand cDNA (Omniscript ${ }^{\circledR}$ RT Kit; Qiagen) as detailed previously [19] utilizing an ABI-PRISM ${ }^{\circledR} 7900$ HT Sequence Detection System. mRNA abundance was calculated using the comparative $\mathrm{C}_{\mathrm{T}}$ method: $\Delta \mathrm{C}_{\mathrm{T}}=\mathrm{C}_{\mathrm{T}}$ [gene of interest $]-\mathrm{C}_{\mathrm{T}}$ [endogenous control $]$ and $\Delta \Delta \mathrm{C}_{\mathrm{T}}=\Delta \mathrm{C}_{\mathrm{T}}[$ Diff CTRL cells $]-\Delta \mathrm{C}_{\mathrm{T}}$ [treated cells]. The fold change for the gene of interest was expressed as $2^{-\Delta \Delta \mathrm{C}_{\mathrm{T}}}$.

\section{Adipokine and cytokine secretion (Luminex)}

Supernatants of differentiating adipocytes were collected at day 8 (after 5 days conditioning, day 4-8) of the treatment period and stored at $-80^{\circ} \mathrm{C}$ till analysis. MILLIPLEX MAP Human Adipocyte Panel (Cat\#HADCYT-61K) kits were purchased from Millipore (Billerica, Massachusetts, USA) and used according to the manufacture's protocol on the LiquiChip ${ }^{\circledR}$ Workstation IS 200 (Luminex technology; Qiagen, Hilden, Germany). Detected molecules were: adiponectin (Adipo), hepatic growth factor (HGF), interleukin (IL)-6, IL-8/CXCL8, monocyte chemoattractant protein (MCP)1/CCL2 and plasminogen activator inhibitor (PAI)-1 (active, serpin E1). Measurements were run in triplicates and final concentrations were normalized by nucleus count per well. Data evaluation was performed with the LiquiChip ${ }^{\circledR}$ Analyser software from Qiagen. 


\section{Statistical analysis}

In brief, data points from repetitive experiments conducted with pre-adipocytes from the same donor were set relative to the corresponding Diff CTRL mean $(=100 \%)$ and averaged. Subsequently all relative values from the different donors and super lot (Supplemental Table S3.1) were used for calculating the overall mean \pm SEM. Statistical significance of the mean differences between treatment and Diff CRTL was tested by a linear mixed model or Student's t-test. P values $<0.05$ were considered significant. ArrayScan ${ }^{\mathrm{TM}}$ results and cytokine concentrations are shown as relative mean \pm SEM. Gene expression data are expressed as fold change (FC) \pm error (based on SEM). For details of the statistical analysis applied to the three data sets see Supplemental Method S3.4.

\section{Results}

\section{Combinations of bio-active dietary constituents inhibited lipid accumulation}

To determine effects of bio-active dietary constituents on lipid accumulation in adipocytes, preadipocytes were differentiated for 14 days in the presence or absence of the compounds. Subsequently, LD accretion in the mature adipocytes was assessed by fluorescent microscopy (Figure 3.1A). Lyc alone decreased the LD-area by $15 \%$ at $0.5 \mu \mathrm{M}(\mathrm{p}=0.021)$ and the LD-intensity by $20 \%$ at $2 \mu \mathrm{M}(\mathrm{p}<0.05)$, while Res and EPA alone did not affect lipid accumulation (Figure 3.1B) compared to Diff CTRL. However, the combination Lyc/Res substantially reduced the adipocyte lipid content (represented by shown LD-parameters). Lyc/Res at $2 / 25 \mu \mathrm{M}$, significantly inhibited LD-number, -area and -intensity by 25\% $(\mathrm{p}<0.001), 17 \%(\mathrm{p}<0.01)$ and $23 \%(\mathrm{p}=0.044)$, respectively (Figure 3.1A+C, right), in comparison with the Diff CTRL. In addition, combined treatment with EPA/Res at $25 / 25 \mu \mathrm{M}$ reduced LD-area and -intensity by $22 \%(\mathrm{p}<0.001)$ and $26 \%$ $(p=0.011)$, respectively, whereas the LD-number was slightly attenuated (Figure 3.1C). Together, these data indicate that the combination of Lyc/Res and EPA/Res significantly reduced the adipocyte lipid content compared to Diff CTRL. 


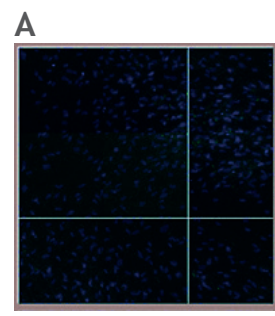

CTRL

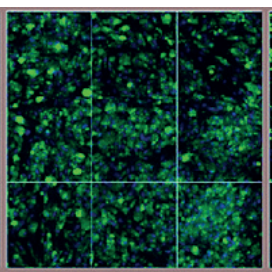

Diff CTRL

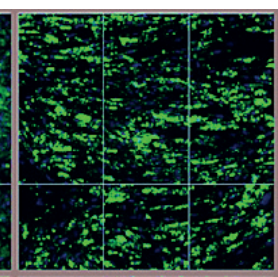

Res [25]

concentration [microM]

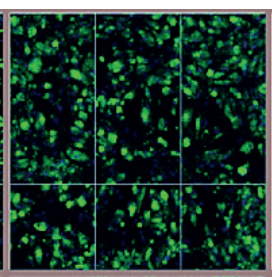

Lyc [2]

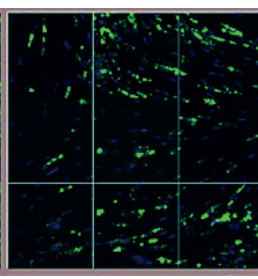

Lyc/Res [2/25]

B

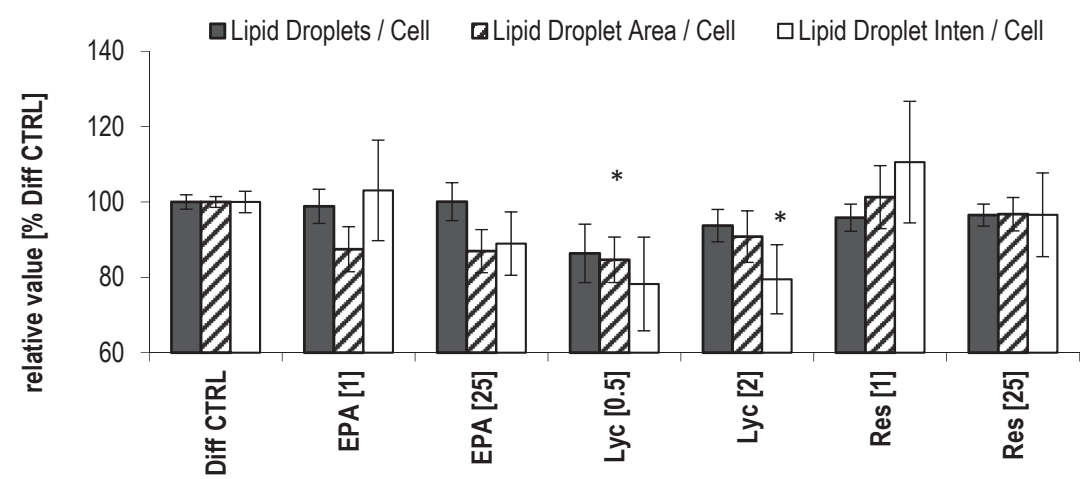

C

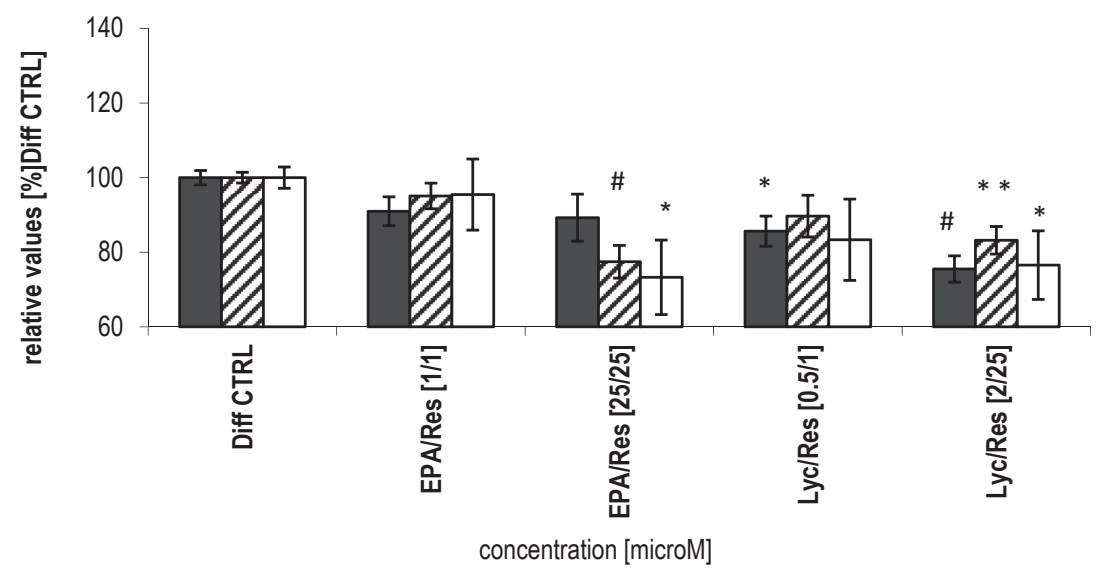

Figure 3.1: Effects of lycopene (Lyc), resveratrol (Res), and eicosapentaenoic acid (EPA) on lipid droplet parameters in differentiated human adipocytes

Primary human pre-adipocytes (HPAd) were differentiated for 14 days in the presence of bio-active compounds. (A) Representative fluorescent images of cells after growth in non-differentiation medium (CTRL) and differentiation-only medium in the absence (Diff CTRL) or presence of resveratrol (Res), lycopene (Lyc) and Lyc/Res at high concentrations. (B) Quantification of the effects of Lyc, Res and eicosapentaenoic acid (EPA) alone and (C) of the combinations of EPA/Res and Lyc/Res on lipid droplet features. Shown are three parameters (Lipid Droplets/Cell, Lipid Droplet Area/Cell and Lipid Droplet Intensity/Cell) that quantify lipid accumulation in adipocytes, as compared to Diff CTRL set as 100\%. Data are represented as overall mean \pm SEM ( 7 donors, $n \geq$ 6). $\left(^{*}\right) p<0.05,\left({ }^{*}\right) p<0.01,(\#) p<0.001$ (versus Diff CTRL, linear mixed model). 


\section{Dietary bio-actives affected adipogenic, lipogenic and lipolytic gene expression}

In order to investigate the possible underlying mechanism for this attenuated lipid accumulation targeted quantitative RT-PCR analysis was performed at day 8 of differentiation, a time point intermediate between undifferentiated and differentiated adipocytes. The combinations EPA/Res and Lyc/Res at high concentrations increased the expression of the adipogenic master regulator, PPAR-gamma, by 1.9-fold and 1.4-fold ( $\mathrm{p}<0.001$ ), respectively, compared with Diff CTRL (Figure 3.2A). Lyc/Res reduced mRNA levels of the lipogenic genes FAS (fatty acid synthase) and GLUT-4 (glucose transporter type 4/SLC2A4) significantly (Figure 3.2E+F, p<0.05). In addition, the combination of EPA/Res strongly blunted the lipogenic markers LPL (lipoprotein lipase) and GLUT-4 mRNA (0.03 and 0.15-fold, respectively; Figure 3.2C+F, p<0.001). Both combinations did not affect FABP-4 (cytosolic fatty acid binding protein 4) expression (Figure 3.2D). Res alone at $25 \mu \mathrm{M}$ moderately enhanced the expression of PPAR-gamma to 1.7-fold $(p<0.001)$ and reduced GLUT-4 expression 0.7 -fold $(p=0.007)$ compared to Diff CTRL. The expression of C/EBP-alpha, LPL and GLUT-4 was diminished to 0.5-fold, 0.1-fold, and 0.4-fold, respectively, when pre-adipocytes were differentiated in the presence of $25 \mu \mathrm{M}$ EPA (Figure 3.2B, $\mathrm{C}+\mathrm{F}, \mathrm{p}<0.01$ ). In contrast, Lyc alone (at $2 \mu \mathrm{M})$ did not affect adipogenic and lipogenic gene expression (Figure 3.2A-F).

The mRNA levels of the major lipases HSL and ATGL were neither significantly affected by Lyc and EPA nor by the combinations, although Res alone increased ATGL expression 2.6-fold ( $p=0.006$; Figure $3.2 \mathrm{G}+\mathrm{H}$ ) in comparison to Diff CTRL. However, combining these bio-active compounds significantly diminished the expression of the LD covering protein PLIN-1 (perilipin 1; Figure 3.2I, EPA/Res: 0.2-fold and Lyc/Res: 0.4-fold, $p<0.001$ ). A comparable effect on the expression of lipogenic and lipolytic genes was observed at day 14 for both combinations (Supplemental Figure S3.2). Altogether, these results demonstrate that combining of Lyc/Res and EPA/Res affects the balance between adipogenic, lipogenic and lipolytic gene expression in white human adipocytes.

\section{Bio-active compounds attenuated secretion of pro-inflammatory cytokines}

Finally, we analysed the influence of dietary bio-actives on cyto-/adipokine secretion of differentiating human adipocytes. EPA alone and the combination EPA/Res strongly reduced the secretion of the insulin sensitizer Adipo by $\sim 90 \%$ whereas Res and Lyc/Res blunted it by $\sim 60 \%$ (Figure 3.3A) compared to Diff CTRL. 
Adipogenic transcription factors

A

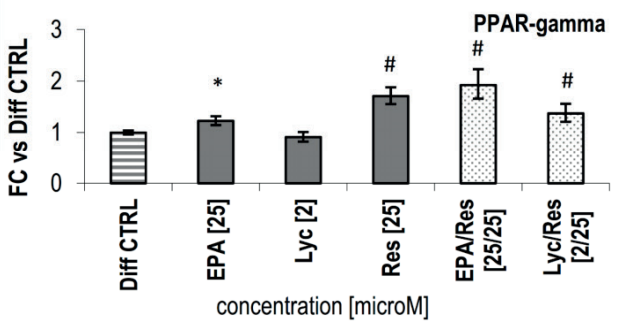

B

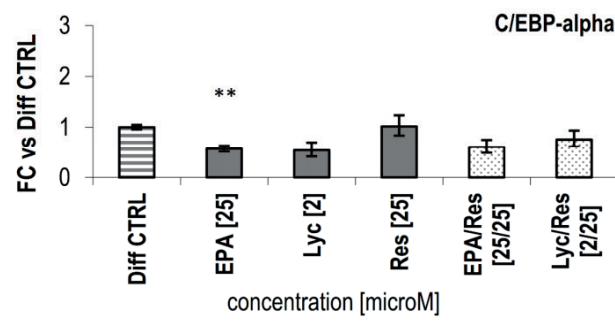

Lipogenesis markers

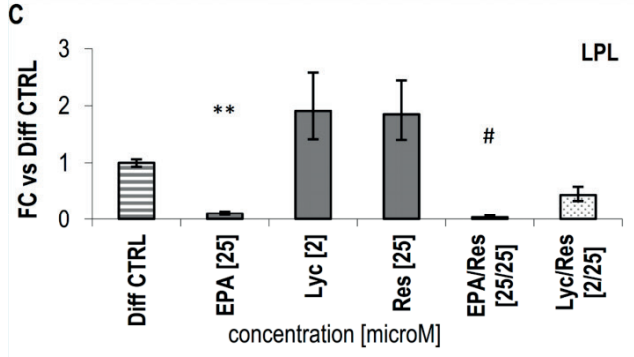

$$
\text { D }
$$

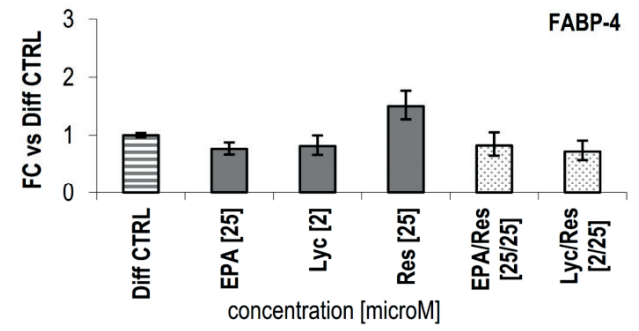

E

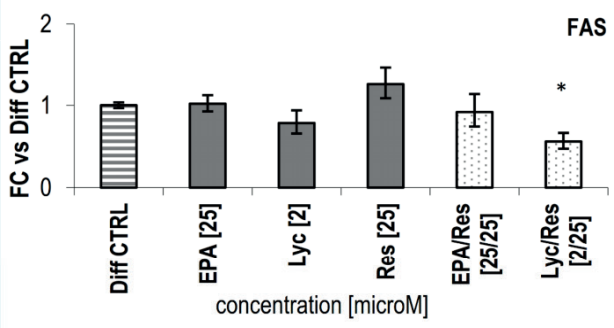

F

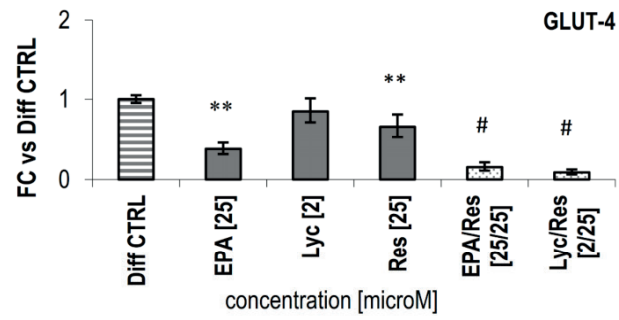

Lipolysis associated proteins

G

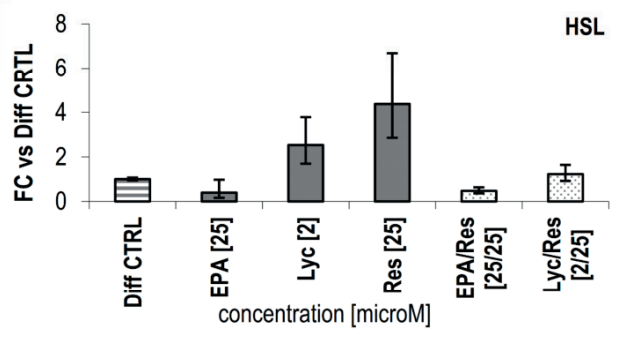

I

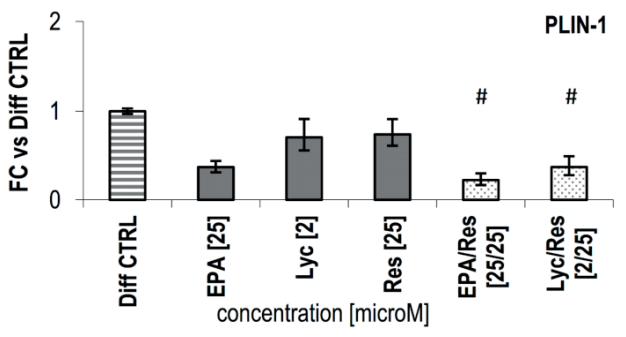

H

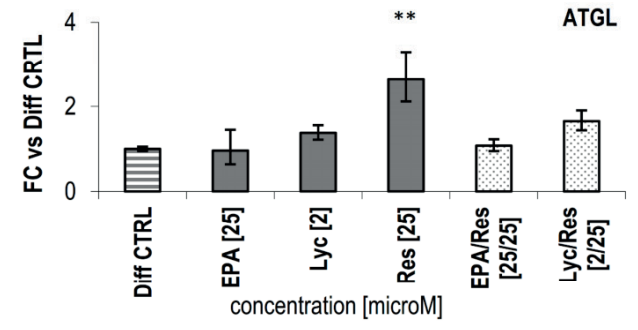




\section{Figure 3.2: Effects of bio-active compounds on adipogenic, lipogenic and lipolytic gene expression markers}

Displayed are the effects of EPA, Lyc and Res or combinations thereof on gene expression after 8 days treatment of HPAd. mRNA levels of adipogenic transcription factors PPAR-gamma $(A)$ and C/EBP-alpha (B), lipogenesis markers LPL (C), FABP-4 (D), FAS (E) and GLUT-4 (F) and lipolytic markers HSL (G), ATGL (H) and PLIN-1 (I) were determined by quantitative RT-PCR. Data are shown as crude fold change (FC) \pm error (based on SEM, 2 donors, 1 super lot, $n \geq 2$ ), compared to Diff CTRL set as 1. $\left({ }^{*}\right) p<0.05$, $\left.{ }^{* *}\right) p<0.01$, (\#) $p<0.001$ (versus Diff CTRL, linear mixed model). CV for dCT values: $<5 \%$ for all genes.

The combinations EPA/Res and Lyc/Res and EPA suppressed pro-inflammatory IL-6 secretion by $70 \%$. Release of the pro-inflammatory cytokine MCP-1 was significantly reduced by more than $30 \%$ by all compounds and combinations except Res. Both combinations caused a similar secretion pattern for the above mentioned cyto-/adipokines at day 14 of differentiation (Supplemental Figure S3.2). Further, the secretion of angiogenic HGF was markedly diminished by Lyc/Res $(70 \%$, $\mathrm{p}<0.01$ ), whereas PAI-1 levels were not modified by the compounds (Figure 3.3E+F) in comparison to the Diff CTRL.

Together, our data indicate that EPA and the combinations Lyc/Res and EPA/Res are able to suppress the secretion of pro-inflammatory markers, but concurrently attenuate the antiinflammatory and insulin sensitizing adipocyte-specific hormone adiponectin.

\section{Discussion}

Weight gain is accompanied by increased lipid storage in AT, altered gene expression and production of pro- and anti-inflammatory mediators [20]. In the present study, we examined the effect of the three abundantly consumed bio-actives Lyc, Res, and EPA on human white adipocyte function and whether combining Res with EPA or Lyc has amplifying effects. Our in-vitro data demonstrate that combining Lyc with Res caused a significant reduction in LD-number, -area and -intensity in human adipocytes. This reduced lipid accumulation was accompanied by increased expression of adipogenic and reduced expression of lipogenic and lipolytic markers, and by an attenuated pro-inflammatory secretion profile (i.e. IL-6 and MCP-1).

Single bio-active dietary constituents are known to influence pre-adipocyte differentiation, proliferation, and adipocyte function directly or indirectly [15-18]. Surprisingly, in our study Res and EPA treatment alone showed no significant effect on the LD accumulation in human adipocytes. This is contrary to the general reported findings in in-vitro and animal studies, which suggest that Res and EPA are capable of diminishing lipid storage in rodent adipocytes [21-24]. 
A

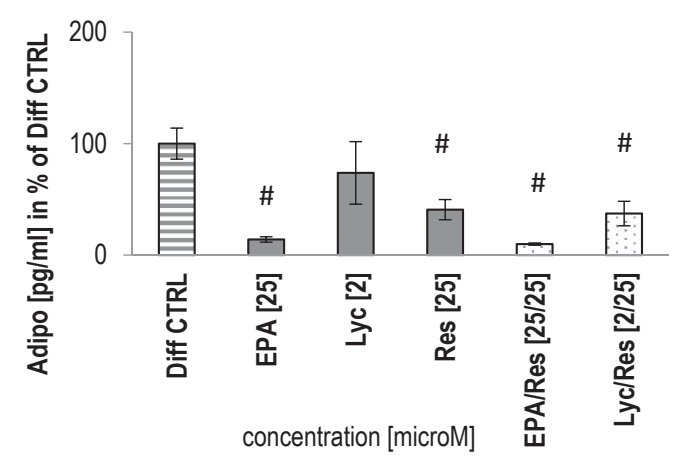

C

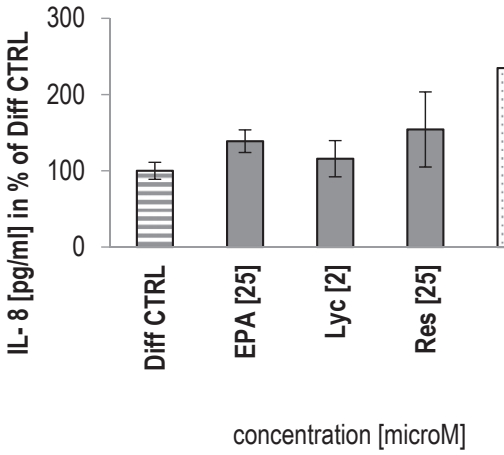

$\mathrm{E}$

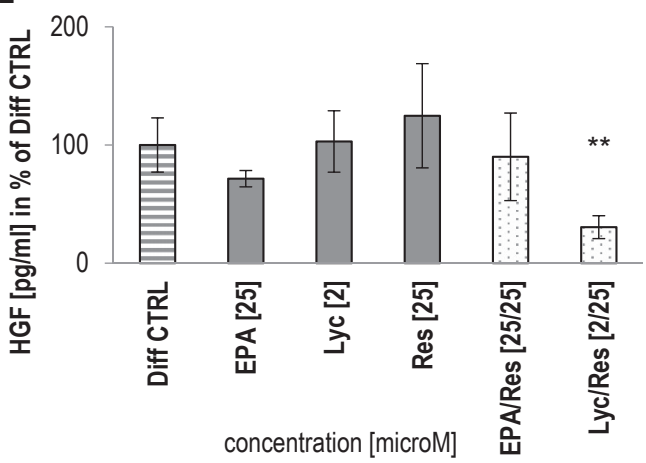

B

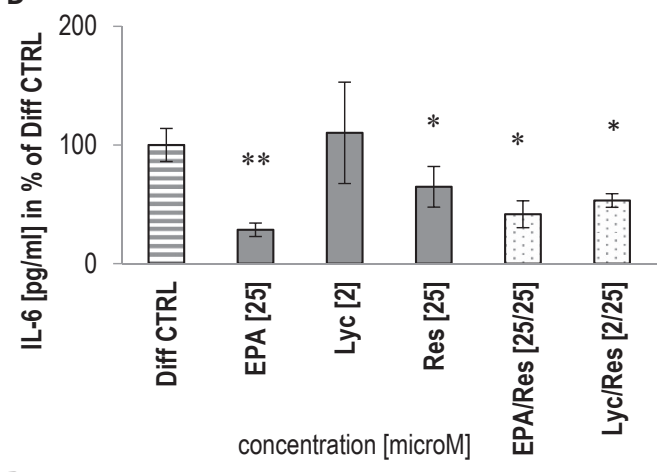

D

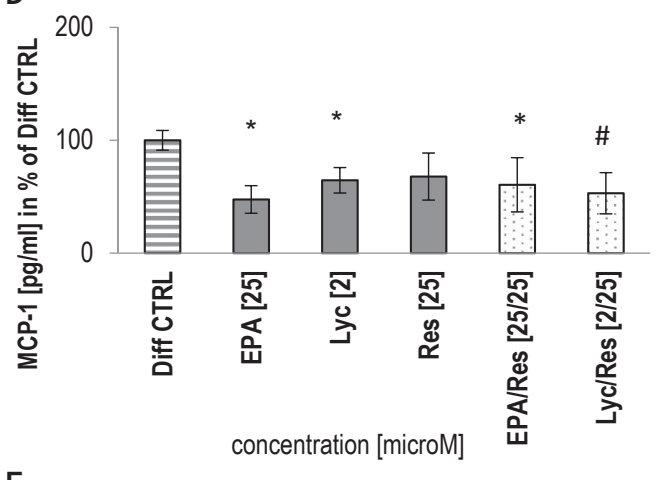

$\mathbf{F}$

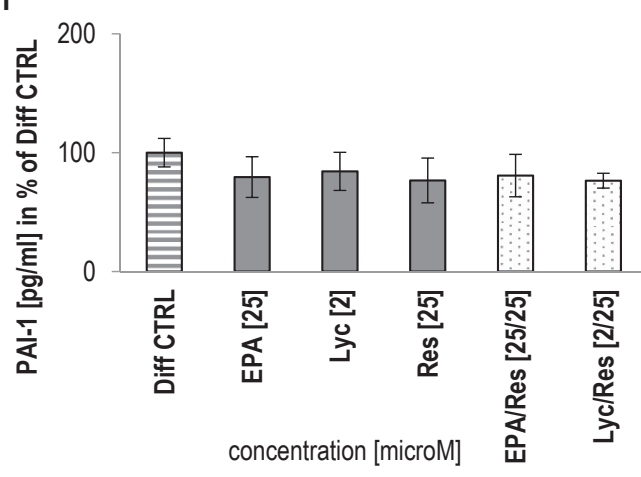

Figure 3.3: Adipokine and cytokine secretion of adipocytes treated with bio-active compounds

Supernatants of HPAd, cultured in the presence or absence of EPA, Lyc and Res or combinations thereof, were collected at day 8 of differentiation and subsequently analysed on the LiquiChip ${ }^{\circledR}$ workstation. The accumulation of the following adipokines: adiponectin (Adipo, A), interleukin-6 (IL-6, B) and 8 (IL-8, C), monocyte chemotactic protein 1 (MCP-1, D), hepatic growth factor (HGF, E) and plasminogen activator inhibitor-1 (PAl-1, F) was measured in media after 5 days conditioning (day 4-8), as compared to Diff CTRL set as $100 \%$. Data are shown for three independent experimental series as overall mean $\left.\pm \mathrm{SEM} .\left(^{*}\right) p<0.05,{ }^{* *}\right)$ $p<0.01$, (\#) $p<0.001$ (versus Diff CTRL, linear mixed model). 
However, in human adipocytes lipid accumulation was either promoted or unaffected by EPA [25, 26], what seems in line with our observation after 14 days treatment. For Res, both enhancing and suppressive effects on lipid accumulation were described in murine 3T3-L1 cells and following a high fat diet (HFD) in mice [27, 28]. In human Simpson-Golabi-Behmel syndrome (SGBS) adipocytes anti-adipogenic effects of Res were reported for concentrations $>30 \mu \mathrm{M}[29,30]$, suggesting that Res at $25 \mu \mathrm{M}$ (this study) might be at switch between its pro- and anti-adipogenic actions. The limited number of human clinical trials with Res or EPA supplementation showed controversial results regarding their effects on adipocyte size, function [31,32] and adipose inflammation [33, 34], possible due to variation in population, dose, and duration of supplementation. Together, these data indicate that further research is necessary to elucidate the exact effect of Res and EPA on adipogenic potential of human (pre-)adipocytes, under the consideration that the usage of primary white adipocytes can affect the experimental outcomes due to intrinsic donor characteristics [35].

Supplementation with the carotenoid Lyc can increase its levels in human AT [36] and 3T3-L1 cells [37] and it is well studied for its anti-oxidative effects [38]. In addition, our results show for the first time that Lyc alone moderately but significantly reduces lipid accumulation in human adipocytes. This effect was also observed in C3H10 T1/2 adipocytes [19]. Conversely, Lyc or its metabolite apo-10'-lycopenoic acid (APO10LA) did not modulate AT mass in rodents [39] (following oral administration for 6 weeks) [40]. In contrast to the minor effect of single compounds, our data clearly demonstrate that the combination Lyc/Res has a stronger effect on LD accumulation, suggesting that Res enhances Lyc's modest anti-adipogenic effect.

Although not a direct measure of lipid turn-over within adipocytes, investigating mRNA levels of genes involved in related pathways could provide possible underlying mechanisms for the amplifying effects observed with combinations of bio-actives. Res's anti-adipogenic properties may be attributed to its effects on both master regulators of adipogenesis, PPAR-gamma and C/EBP-alpha, which are repressed via activation of AMP-activated protein kinase (AMPK) [41]. Interestingly, in the present study Res at $25 \mu \mathrm{M}$ up-regulated PPAR-gamma expression and showed no effect on C/EBP-alpha mRNA in differentiating human adipocytes. This seems contrary to the reported down-regulation of PPAR-gamma and lipogenic markers in 3T3-L1 [41] and SGBS cells [29] at concentrations $>25 \mu \mathrm{M}$ and the accompanying reduction of fat pads in mice following 10 weeks of HFD feeding [42]. We hypothesize that in the present study the concentration of Res $(25 \mu \mathrm{M})$ is insufficient to counteract the strong adipogenic effects of the PPAR-gamma agonist rosiglitazone [43], contained in the media at $100 \mu \mathrm{M}$ during the complete 
differentiation period. This is further supported by results in differentiating SGBS cells, demonstrating no effect of $20 \mu \mathrm{M}$ Res on PPAR-gamma expression even in the presence of a low rosiglitazone concentration $(2 \mu \mathrm{M})$ [29].

PUFAs can act as PPAR agonists and display PPAR-independent gene control function [44]. Regarding EPA, a well-known n-3 PUFA, a reduction in stored LDs and a concomitant modification of adipogenic markers, e.g. a decrease of PPAR-gamma expression, was described in murine fat cells $[19,21]$. However, in line with our results, an upregulation of PPAR-gamma expression is described in isolated human adipocytes [45], suggesting species-specific effects of EPA on adipogenesis. Finally, Lyc is not presented as an adipogenic modulator [16], however some conflicting data exist regarding its impact on adipose gene expression. In accordance with our human data, in murine adipocytes, Lyc treatment did not affect adipogenic genes [39] but moderately decreased lipid deposition [19], whereas Lyc administration for 6 weeks decreased PPAR-gamma mRNA in AT of rats without changes in total fat mass [46].

In addition to adipogenesis, the balance between lipogenesis and lipolysis largely influences total lipid amount in human adipocytes. The combination of Lyc/Res and EPA/Res strongly suppressed the lipogenic markers GLUT-4, LPL and FAS in our human fat cells. However, for the single compounds conflicting effects on these markers were reported previously. Supporting our results, a reduction of GLUT-4 mRNA and unaffected levels of FAS mRNA were shown in 3T3-L1 cells after Res $(20 \mu \mathrm{M})$ [47] and EPA $(100 \mu \mathrm{M})$ [48] treatment, respectively. Whereas, opposing to our human data, GLUT-4 and LPL mRNA expression was up-regulated after treating murine adipocytes with Lyc $(2 \mu \mathrm{M})[19]$ and EPA $(100 \mu \mathrm{M})$ [21], respectively.

Furthermore, the lipases ATGL and HSL and the LD-covering protein PLIN-1 are key proteins in the intracellular lipolytic process, being tightly regulated by neuroendocrine signals (reviewed in [49]). Our results are in agreement with Lasa et al. [50], who reported that the mRNA level of ATGL but not HSL, were increased following treatment of mature human and murine adipocytes with $100 \mu \mathrm{M}$ Res for 24h, which was accompanied by an increased FFA release [50]. Interestingly, the mRNA of the dominant LD-covering protein PLIN-1 was not altered by the single constituents. In line, a recent in-vitro study in human adipocytes also demonstrated that EPA alone had no impact on PLIN-1 expression [51]. On the other hand, the combinations significantly suppressed PLIN-1 mRNA in our human adipocyte model, suggesting interactions between bio-active compounds which influence PLIN-1 expression. However, it needs to be investigated whether this translates into functional changes in the lipolytic potential of the adipocytes. 
Together, the pronounced down-regulation of lipogenic (GLUT-4, LPL, FAS) and lipolytic genes (PLIN-1) by the combination of bio-active compounds (EPA and Lyc with Res) cannot be justified by additive effects of the single compounds, suggesting strong amplifying/synergistic interactions. Therefore, combining bio-active constituents facilitates the interplay of different direct and indirect transcriptional controlling mechanisms, which warrants further investigations.

Finally, we showed that the reduction in lipid accumulation following EPA/Res or Lyc/Res treatment was accompanied by a diminished release of the pro-inflammatory cytokines IL- 6 and MCP-1. In line, a previous study, in different adipocyte models and in murine AT explants, demonstrated that pre-incubation with the anti-inflammatory nutrient Lyc $(24 \mathrm{~h}, 2 \mu \mathrm{M})$ reduced the secretion of the pro-inflammatory cytokines IL-6 and MCP-1 induced by tumour necrosis factor (TNF)-alpha stimulation or HFD-feeding, respectively [52]. Additionally, a study with HFDinduced obese rats showed that Lyc supplementation increased plasma adiponectin levels and decreased the mRNA of MCP-1 and IL-6 in AT and their circulating protein levels [40]. Without stimulation of the inflammatory pathways (this study), Lyc only suppressed the secretion of the macrophage attracting chemokine MCP-1 [53] from human adipocytes. Furthermore, several studies indicate that treatment of mature SGBS and 3T3-L1 adipocytes with Res (100 $\mu \mathrm{M}, 48 \mathrm{~h})$ $[30,54]$ and EPA $(200 \mu \mathrm{M}, 48 \mathrm{~h})[55,56]$, respectively, and supplementation of HFD-fed mice with both bio-actives (Res, 10 weeks [42]; EPA, 5 or 11 weeks $[55,56]$ ) can reduce the expression and secretion of pro-inflammatory cytokines such as MCP-1, IL-6 and IL-8 in AT.

In addition, we observed a decrease of Adipo secretion with Res, EPA and the combinations. However, several groups reported an increase of the anti-inflammatory and insulin sensitizing adipokine adiponectin and a decrease of leptin expression and secretion after treatment of mature 3T3-L1- and SGBS-adipocytes with Res (up to $200 \mu \mathrm{M}$ ) or its metabolites for 24 or $48 \mathrm{~h}$, respectively [30, 57]. Similarly, EPA treatment $(100 \mu \mathrm{M}, 24$ or $48 \mathrm{~h})$ was also able to increase Adipo secretion from mature primary human adipocytes $[26,58]$. Nevertheless, our findings are in line with recent data from Lorente-Cebrian et al. [59], who reported that $200 \mu \mathrm{M}$ EPA (96 h) significantly decreased adiponectin gene expression and protein secretion in freshly isolated rat adipocytes [59]. Furthermore, we noticed a strong attenuation of the angiogenic protein HGF by the combination Lyc/Res. HGF is secreted from AT and its expression is elevated in the obese state allowing for remodelling of AT when expanding [60] and exhibits anti-inflammatory characteristics on the cross-talk between macrophages and adipocytes in mice [61].

Altogether our data suggest that treatment with dietary bio-actives during adipogenic differentiation, alters the cyto-/adipokine release towards a less pro-inflammatory secretion 
profile, indicated by an attenuated IL-6 and MCP-1 release. The combinations EPA/Res and Lyc/Res partially augmented the effects of the single compounds on the secretion of adiponectin, IL-6, MCP-1 and HGF, supporting the concept of strong amplifying/synergistic interactions between nutrients [62].

\section{Conclusions}

Here we show for the first time, that the combination of the dietary bio-actives Lyc and EPA with Res might influence adipocyte function synergistically by affecting the balance between adipogenic, lipogenic and lipolytic gene expression, resulting in a reduced lipid accumulation and improved inflammatory profile. Our data suggest that applying combinations of bio-actives is a more favourable approach to tackle AT dysfunction because anti-lipogenic and anti-inflammatory effects can be magnified compared to the single dietary constituents [63-66]. Given that obesity is recognized as a state of chronic 'low-grade inflammation' $[10,11]$, reducing the secretion of proand stimulating anti-inflammatory adipokines with combinations of dietary bio-actives could contribute to manage and treat obesity-related metabolic complications.

\section{Abbreviations}

Adipo: adiponectin; AT: adipose tissue; ATGL: adipose triglyceride lipase; bFGF: basic fibroblast growth factor; C/EBP-alpha: CCAAT/enhancer binding protein alpha; DM: differentiation medium; EPA: eicosapentaenoic acid; FA: fatty acid; FABP-4: cytosolic fatty acid binding protein 4; FAS: fatty acid synthase; FC: fold change; FCS: fetal calf serum; GLUT-4/SLC2A4: glucose transporter type 4; GM: growth medium; HFD: high fat diet; HGF: hepatic growth factor; HPAd: human pre-adipocytes; HSL: hormone sensitive lipase; IL-6: interleukin-6; IL-8: interleukin-8; LD: lipid droplet; Lyc: (all-E)-lycopene; LPL: lipoprotein lipase; MCP-1/ CCL2: monocyte chemoattractant protein-1; n: number of experimental series; PAI-1: plasminogen activator inhibitor; PBS: phosphate-buffered saline; PLIN-1: perilipin 1; PPAR-gamma: Peroxisome Proliferator-Activated Receptor Gamma; PUFA: polyunsaturated fatty acid; Res: transresveratrol; SEM: standard error of the mean; SGBS: Simpson-Golabi-Behmel syndrome; TAGs: triacylglycerides.

\section{Acknowledgements}

We would like to thank Nathalie Richard and Albine Bompard for helping to perform the LiquiChip ${ }^{\circledR}$ experiments, Nicole Seifert for cell culture support, Eliane Wandeler and Ann Sion for assistance with the ArrayScan ${ }^{\circledR}$ reader and protocol establishment and Giorgio La Fata and Timo Lischke for critical review of the manuscript. 


\section{References}

1. Vandevijvere S, Chow CC, Hall KD, Umali E, Swinburn BA: Increased food energy supply as a major driver of the obesity epidemic: a global analysis. Bulletin of the World Health Organization 2015, 93(7):446-456.

2. Guilherme A, Virbasius JV, Puri V, Czech MP: Adipocyte dysfunctions linking obesity to insulin resistance and type 2 diabetes. Nat Rev Mol Cell Biol 2008, 9(5):367-377.

3. Arner E, Westermark PO, Spalding KL, Britton T, Ryden M, Frisen J, Bernard S, Arner P: Adipocyte turnover: relevance to human adipose tissue morphology. Diabetes 2010, 59(1):105-109.

4. Arner P, Bernard S, Salehpour M, Possnert G, Liebl J, Steier P, Buchholz BA, Eriksson M, Arner E, Hauner $\mathrm{H}$ et al: Dynamics of human adipose lipid turnover in health and metabolic disease. Nature 2011, 478(7367):110-113.

5. Hammarstedt A, Graham TE, Kahn BB: Adipose tissue dysregulation and reduced insulin sensitivity in non-obese individuals with enlarged abdominal adipose cells. Diabetology \& metabolic syndrome 2012, 4:42-42.

6. Arner P, Langin D: Lipolysis in lipid turnover, cancer cachexia, and obesity-induced insulin resistance. Trends in endocrinology and metabolism: TEM 2014, 25(5):255-262.

7. Rosen ED, MacDougald OA: Adipocyte differentiation from the inside out. Nat Rev Mol Cell Biol 2006, 7(12):885-896.

8. Weisberg SP, McCann D, Desai M, Rosenbaum M, Leibel RL, Ferrante AW, Jr.: Obesity is associated with macrophage accumulation in adipose tissue. The Journal of clinical investigation 2003, 112(12):1796-1808.

9. Galic S, Oakhill JS, Steinberg GR: Adipose tissue as an endocrine organ. Molecular and cellular endocrinology 2010, 316(2):129-139.

10. Kim CS, Park HS, Kawada T, Kim JH, Lim D, Hubbard NE, Kwon BS, Erickson KL, Yu R: Circulating levels of MCP-1 and IL-8 are elevated in human obese subjects and associated with obesity-related parameters. International journal of obesity 2006, 30(9):1347-1355.

11. Calder PC, Ahluwalia N, Brouns F, Buetler T, Clement K, Cunningham K, Esposito K, Jonsson LS, Kolb H, Lansink M et al: Dietary factors and low-grade inflammation in relation to overweight and obesity. The British journal of nutrition 2011, 106 Suppl 3:S5-78.

12. Hsu CL, Yen GC: Phenolic compounds: evidence for inhibitory effects against obesity and their underlying molecular signaling mechanisms. Molecular nutrition \& food research 2008, 52(1):53-61.

13. Madsen L, Petersen RK, Kristiansen K: Regulation of adipocyte differentiation and function by polyunsaturated fatty acids. Biochimica et biophysica acta 2005, 1740(2):266-286.

14. Kawada T, Kamei Y, Fujita A, Hida Y, Takahashi N, Sugimoto E, Fushiki T: Carotenoids and retinoids as suppressors on adipocyte differentiation via nuclear receptors. BioFactors 2000, 13(14):103-109.

15. Wang S, Moustaid-Moussa N, Chen L, Mo H, Shastri A, Su R, Bapat P, Kwun I, Shen CL: Novel insights of dietary polyphenols and obesity. The Journal of nutritional biochemistry 2014, 25(1):118.

16. Luisa Bonet M, Canas JA, Ribot J, Palou A: Carotenoids and their conversion products in the control of adipocyte function, adiposity and obesity. Archives of biochemistry and biophysics 2015, 572:112125.

17. Aguirre L, Fernández-Quintela A, Arias N, Portillo M: Resveratrol: Anti-Obesity Mechanisms of Action. Molecules 2014, 19(11):18632.

18. Martínez-Fernández L, Laiglesia LM, Huerta AE, Martínez JA, Moreno-Aliaga MJ: Omega-3 fatty acids and adipose tissue function in obesity and metabolic syndrome. Prostaglandins \& other lipid mediators.

19. Warnke I, Goralczyk R, Fuhrer E, Schwager J: Dietary constituents reduce lipid accumulation in murine C3H10 T1/2 adipocytes: A novel fluorescent method to quantify fat droplets. Nutrition \& metabolism 2011, 8(1):30.

20. Alligier M, Meugnier E, Debard C, Lambert-Porcheron S, Chanseaume E, Sothier M, Loizon E, Hssain AA, Brozek J, Scoazec JY et al: Subcutaneous adipose tissue remodeling during the initial phase of weight gain induced by overfeeding in humans. The Journal of clinical endocrinology and metabolism 2012, 97(2):E183-192. 
21. Manickam E, Sinclair AJ, Cameron-Smith D: Suppressive actions of eicosapentaenoic acid on lipid droplet formation in 3T3-L1 adipocytes. Lipids in health and disease 2010, 9(1):57.

22. Rayalam S, Yang JY, Ambati S, Della-Fera MA, Baile CA: Resveratrol induces apoptosis and inhibits adipogenesis in 3T3-L1 adipocytes. Phytotherapy research : PTR 2008, 22(10):1367-1371.

23. Bertrand C, Pignalosa A, Wanecq E, Rancoule C, Batut A, Deleruyelle S, Lionetti L, Valet P, CastanLaurell I: Effects of Dietary Eicosapentaenoic Acid (EPA) Supplementation in High-Fat Fed Mice on Lipid Metabolism and Apelin/APJ System in Skeletal Muscle. PloS one 2013, 8(11):e78874.

24. Alberdi G, Rodríguez VM, Miranda J, Macarulla MT, Arias N, Andrés-Lacueva C, Portillo MP: Changes in white adipose tissue metabolism induced by resveratrol in rats. Nutrition \& metabolism 2011, 8:29-29.

25. Hanada H, Morikawa K, Hirota K, Nonaka M, Umehara Y: Induction of apoptosis and lipogenesis in human preadipocyte cell line by n-3 PUFAs. Cell biology international 2011, 35(1):51-59.

26. Romacho T, Glosse P, Richter I, Elsen M, Schoemaker MH, van Tol EA, Eckel J: Nutritional ingredients modulate adipokine secretion and inflammation in human primary adipocytes. Nutrients 2015, 7(2):865-886.

27. Hu P, Zhao L, Chen J: Physiologically achievable doses of resveratrol enhance 3T3-L1 adipocyte differentiation. European journal of nutrition 2015, 54(4):569-579.

28. Chang CC, Lin KY, Peng KY, Day YJ, Hung LM: Resveratrol exerts anti-obesity effects in high-fat diet obese mice and displays differential dosage effects on cytotoxicity, differentiation, and lipolysis in 3T3-L1 cells. Endocrine journal 2015.

29. Fischer-Posovszky P, Kukulus V, Tews D, Unterkircher T, Debatin KM, Fulda S, Wabitsch M: Resveratrol regulates human adipocyte number and function in a Sirt1-dependent manner. Am J Clin Nutr 2010, 92(1):5-15.

30. Rosenow A, Noben JP, Jocken J, Kallendrusch S, Fischer-Posovszky P, Mariman EC, Renes J: Resveratrol-induced changes of the human adipocyte secretion profile. Journal of proteome research 2012, 11(9):4733-4743.

31. Yoshino J, Conte C, Fontana L, Mittendorfer B, Imai S, Schechtman KB, Gu C, Kunz I, Rossi Fanelli F, Patterson BW et al: Resveratrol supplementation does not improve metabolic function in nonobese women with normal glucose tolerance. Cell metabolism 2012, 16(5):658-664.

32. Konings E, Timmers S, Boekschoten MV, Goossens GH, Jocken JW, Afman LA, Muller M, Schrauwen P, Mariman EC, Blaak EE: The effects of 30 days resveratrol supplementation on adipose tissue morphology and gene expression patterns in obese men. International journal of obesity 2014, 38(3):470-473.

33. Skarke C, Alamuddin N, Lawson JA, Li X, Ferguson JF, Reilly MP, FitzGerald GA: Bioactive products formed in humans from fish oils. Journal of lipid research 2015, 56(9):1808-1820.

34. Ferguson JF, Xue C, Hu Y, Li M, Reilly MP: Adipose tissue RNASeq reveals novel gene-nutrient interactions following n-3 PUFA supplementation and evoked inflammation in humans. The Journal of nutritional biochemistry 2016, 30:126-132.

35. Lo Surdo J, Bauer SR: Quantitative approaches to detect donor and passage differences in adipogenic potential and clonogenicity in human bone marrow-derived mesenchymal stem cells. Tissue engineering Part C, Methods 2012, 18(11):877-889.

36. Walfisch Y, Walfisch S, Agbaria R, Levy J, Sharoni Y: Lycopene in serum, skin and adipose tissues after tomato-oleoresin supplementation in patients undergoing haemorrhoidectomy or peri-anal fistulotomy. The British journal of nutrition 2003, 90(4):759-766.

37. Moussa M, Gouranton E, Gleize B, Yazidi CE, Niot I, Besnard P, Borel P, Landrier JF: CD36 is involved in lycopene and lutein uptake by adipocytes and adipose tissue cultures. Molecular nutrition $\&$ food research 2011, 55(4):578-584.

38. Basu A, Imrhan V: Tomatoes versus lycopene in oxidative stress and carcinogenesis: conclusions from clinical trials. Eur J Clin Nutr 2007, 61(3):295-303.

39. Gouranton E, Aydemir G, Reynaud E, Marcotorchino J, Malezet C, Caris-Veyrat C, Blomhoff R, Landrier JF, Ruhl R: Apo-10'-lycopenoic acid impacts adipose tissue biology via the retinoic acid receptors. Biochimica et biophysica acta 2011, 1811(12):1105-1114.

40. Luvizotto RdAM, Nascimento AF, Imaizumi E, Pierine DT, Conde SJ, Correa CR, Yeum K-J, Ferreira ALA: Lycopene supplementation modulates plasma concentrations and epididymal adipose 
tissue mRNA of leptin, resistin and IL-6 in diet-induced obese rats. British Journal of Nutrition 2013, 110(10):1803-1809.

41. Chen S, Li Z, Li W, Shan Z, Zhu W: Resveratrol inhibits cell differentiation in 3T3-L1 adipocytes via activation of AMPK. Canadian journal of physiology and pharmacology 2011, 89(11):793-799.

42. Kim S, Jin Y, Choi Y, Park T: Resveratrol exerts anti-obesity effects via mechanisms involving downregulation of adipogenic and inflammatory processes in mice. Biochemical pharmacology 2011, 81(11):1343-1351.

43. Lehmann JM, Moore LB, Smith-Oliver TA, Wilkison WO, Willson TM, Kliewer SA: An antidiabetic thiazolidinedione is a high affinity ligand for peroxisome proliferator-activated receptor gamma (PPAR gamma). The Journal of biological chemistry 1995, 270(22):12953-12956.

44. Sessler AM, Ntambi JM: Polyunsaturated fatty acid regulation of gene expression. The Journal of nutrition 1998, 128(6):923-926.

45. Chambrier C, Bastard JP, Rieusset J, Chevillotte E, Bonnefont-Rousselot D, Therond P, Hainque B, Riou JP, Laville M, Vidal H: Eicosapentaenoic acid induces mRNA expression of peroxisome proliferator-activated receptor gamma. Obes Res 2002, 10(6):518-525.

46. Luvizotto RA, Nascimento AF, Miranda NC, Wang XD, Ferreira AL: Lycopene-rich tomato oleoresin modulates plasma adiponectin concentration and mRNA levels of adiponectin, SIRT1, and FoxO1 in adipose tissue of obese rats. Human \& experimental toxicology 2015, 34(6):612-619.

47. Mercader J, Palou A, Bonet ML: Resveratrol enhances fatty acid oxidation capacity and reduces resistin and Retinol-Binding Protein 4 expression in white adipocytes. The Journal of nutritional biochemistry 2011, 22(9):828-834.

48. Wójcik C, Lohe K, Kuang C, Xiao Y, Jouni Z, Poels E: Modulation of adipocyte differentiation by omega-3 polyunsaturated fatty acids involves the ubiquitin-proteasome system. Journal of cellular and molecular medicine 2014, 18(4):590-599.

49. Fruhbeck G, Mendez-Gimenez L, Fernandez-Formoso JA, Fernandez S, Rodriguez A: Regulation of adipocyte lipolysis. Nutrition research reviews 2014, 27(1):63-93.

50. Lasa A, Schweiger M, Kotzbeck P, Churruca I, Simon E, Zechner R, Portillo MP: Resveratrol regulates lipolysis via adipose triglyceride lipase. The Journal of nutritional biochemistry 2012, 23(4):379-384.

51. Polus A, Kiec-Wilk B, Razny U, Gielicz A, Schmitz G, Dembinska-Kiec A: Influence of dietary fatty acids on differentiation of human stromal vascular fraction preadipocytes. Biochimica et biophysica acta 2015, 1851(9):1146-1155.

52. Gouranton E, Thabuis C, Riollet C, Malezet-Desmoulins C, El Yazidi C, Amiot MJ, Borel P, Landrier JF: Lycopene inhibits proinflammatory cytokine and chemokine expression in adipose tissue. The Journal of nutritional biochemistry 2011, 22(7):642-648.

53. Kanda H, Tateya S, Tamori Y, Kotani K, Hiasa K, Kitazawa R, Kitazawa S, Miyachi H, Maeda S, Egashira K et al: MCP-1 contributes to macrophage infiltration into adipose tissue, insulin resistance, and hepatic steatosis in obesity. The Journal of clinical investigation 2006, 116(6):1494-1505.

54. Zagotta I, Dimova EY, Debatin KM, Wabitsch M, Kietzmann T, Fischer-Posovszky P: Obesity and inflammation: reduced cytokine expression due to resveratrol in a human in vitro model of inflamed adipose tissue. Frontiers in pharmacology 2015, 6:79.

55. Siriwardhana N, Kalupahana NS, Fletcher S, Xin W, Claycombe KJ, Quignard-Boulange A, Zhao L, Saxton AM, Moustaid-Moussa N: n-3 and n-6 polyunsaturated fatty acids differentially regulate adipose angiotensinogen and other inflammatory adipokines in part via NF-kappaB-dependent mechanisms. The Journal of nutritional biochemistry 2012, 23(12):1661-1667.

56. Kalupahana NS, Claycombe K, Newman SJ, Stewart T, Siriwardhana N, Matthan N, Lichtenstein $\mathrm{AH}$, Moustaid-Moussa N: Eicosapentaenoic acid prevents and reverses insulin resistance in high-fat diet-induced obese mice via modulation of adipose tissue inflammation. The Journal of nutrition 2010, 140(11):1915-1922.

57. Eseberri I, Lasa A, Churruca I, Portillo MP: Resveratrol metabolites modify adipokine expression and secretion in 3T3-L1 pre-adipocytes and mature adipocytes. PloS one 2013, 8(5):e63918.

58. Tishinsky JM, Ma DW, Robinson LE: Eicosapentaenoic acid and rosiglitazone increase adiponectin in an additive and PPARgamma-dependent manner in human adipocytes. Obesity 2011, 19(2):262268. 
59. Lorente-Cebrián S, Pérez-Matute P, Martínez JA, Marti A, Moreno-Aliaga MJ: Effects of eicosapentaenoic acid (EPA) on adiponectin gene expression and secretion in primary cultured rat adipocytes. Journal of physiology and biochemistry 2006, 62(2):61-69.

60. Cao Y: Angiogenesis modulates adipogenesis and obesity. The Journal of clinical investigation 2007 , 117(9):2362-2368.

61. Kusunoki H, Taniyama Y, Otsu R, Rakugi H, Morishita R: Anti-inflammatory effects of hepatocyte growth factor on the vicious cycle of macrophages and adipocytes. Hypertension research : official journal of the Japanese Society of Hypertension 2014, 37(6):500-506.

62. Most J, Goossens GH, Jocken JW, Blaak EE: Short-term supplementation with a specific combination of dietary polyphenols increases energy expenditure and alters substrate metabolism in overweight subjects. International journal of obesity 2014, 38(5):698-706.

63. Rayalam S, Della-Fera MA, Yang JY, Park HJ, Ambati S, Baile CA: Resveratrol potentiates genistein's antiadipogenic and proapoptotic effects in 3T3-L1 adipocytes. The Journal of nutrition 2007, 137(12):2668-2673

64. Park HJ, Yang JY, Ambati S, Della-Fera MA, Hausman DB, Rayalam S, Baile CA: Combined effects of genistein, quercetin, and resveratrol in human and 3T3-L1 adipocytes. Journal of medicinal food 2008, 11(4):773-783.

65. Hadad N, Levy R: The synergistic anti-inflammatory effects of lycopene, lutein, $\beta$-carotene, and carnosic acid combinations via redox-based inhibition of NF- $\kappa \mathrm{B}$ signaling. Free Radical Biology and Medicine 2012, 53(7):1381-1391.

66. Björk C, Wilhelm U, Mandrup S, Larsen BD, Bordoni A, Hedén P, Rydén M, Arner P, Laurencikiene J: Effects of selected bioactive food compounds on human white adipocyte function. Nutrition \& metabolism 2016, 13(1):1-10. 
Supplemental Material 


\section{Supplemental Tables and Methods}

Supplemental Table S3.1: Relevant donor characteristics from used human pre-adipocytes (HPAd)

\begin{tabular}{|l|c|l|c|l|c|c|l|l|}
\hline Cells & Lot \# & ID & Age & Gender & BMI & $\begin{array}{l}\text { Number of } \\
\text { donors }\end{array}$ & $\begin{array}{l}\text { Ethnic } \\
\text { group }\end{array}$ & Biopsy region \\
\hline HPAd & 1375 & Donor 1 & 41 & female & n.g. & 1 & Caucasian & $\begin{array}{l}\text { abdominal, } \\
\text { subcutaneous }\end{array}$ \\
\hline HPAd & 1377 & Donor 2 & 53 & female & n.g. & 1 & Caucasian & $\begin{array}{l}\text { abdominal, } \\
\text { subcutaneous }\end{array}$ \\
\hline $\begin{array}{l}\text { SL0035 } \\
\text { (super lot) }\end{array}$ & 0035 & $\begin{array}{l}\text { Donor } \\
\text { Pool }\end{array}$ & $37-57$ & female & avg 27.9 & 5 & n.g. & $\begin{array}{l}\text { Subcutaneous, } \\
\text { abdominal (3) } \\
\text { and gluteal (2) }\end{array}$ \\
\hline
\end{tabular}

n.g. not given

Supplemental Table S3.2: Altered Cellomics ${ }^{\mathrm{TM}}$ parameters for the analysis of HPAd (20x objective)

\begin{tabular}{|c|c|c|c|c|c|}
\hline \multicolumn{6}{|l|}{ Object Identification } \\
\hline $\begin{array}{l}\text { Channel 1: Objects } \\
\text { Method: }\end{array}$ & \multicolumn{2}{|c|}{ FixedThreshold } & $\begin{array}{l}\text { Channel 2: Spots } \\
\text { Method: }\end{array}$ & \multicolumn{2}{|c|}{ TriangThreshold } \\
\hline Value: & \multicolumn{2}{|l|}{125} & Value: & \multicolumn{2}{|l|}{0.499} \\
\hline Object Selection Parameter & $\underline{\text { Min }}$ & $\underline{\text { Max }}$ & & $\underline{\text { Min }}$ & Max \\
\hline $\begin{array}{l}\text { Channel 1: Objects } \\
\text { ObjectAreaCh1: }\end{array}$ & 50 & 10000 & $\begin{array}{l}\text { Channel 2: Spots } \\
\text { SpotAreaCh2: }\end{array}$ & 3 & 10000 \\
\hline ObjectAvgIntenCh1: & 0 & 4095 & SpotAvgIntenCh2: & 10 & 4095 \\
\hline ObjectTotallntenCh1: & 0 & 4411431044 & SpotTotallntenCh2: & 500 & 4411431044 \\
\hline \multicolumn{6}{|l|}{ Assay Parameters } \\
\hline BackgroundCorrectionCh1: & & 140 & BackgroundCorrectionCh2: & & 140 \\
\hline ObjectSegmentationCh1: & & 20 & CircModifierCh2 & & 127 \\
\hline SmoothFactorCh1: & & 5 & RingDistanceCh2: & & -512 \\
\hline SmoothMethodCh1: & & 1 & SpotDetectRadiusCh2: & & 5 \\
\hline RejectBorderObjectsCh1: & & 0 & SpotSmoothFactorCh2: & & 1 \\
\hline "'PixelSize: & & 4 microns & SpotSmoothMethodCh2: & & 1 \\
\hline
\end{tabular}

Displayed values are pixel number. ThermoFisher Bioapplication applied: SpotDetector ${ }^{\circledR}$ 
Supplemental Table S3.3: Sequences of human primers and probes for adipocyte specific genes used for quantitative RT-PCR

\begin{tabular}{|c|c|c|}
\hline Gene name & $\begin{array}{l}\text { Forward Primer } \\
\text { Reverse Primer }\end{array}$ & $\begin{array}{l}\text { Probe } \\
\text { (FAM conjugated) }\end{array}$ \\
\hline $\begin{array}{l}\text { Peroxisome proliferator activated } \\
\text { receptor gamma 1+2 (PPARy 1+2) } \\
\text { NM_138712.3, NM_015869.4 }\end{array}$ & $\begin{array}{l}\text { 5'TCAGAAATGCCTTGCAGTG'3 } \\
\text { 5'TTCTCGGCCTGTGGCATC'3 }\end{array}$ & 5'TGTCTCATAATGCCATCAGGTTTGGGC'3 \\
\hline $\begin{array}{l}\text { CCAAT/enhancer binding protein } \\
\text { alpha (C/EBPa) NM } 001287435.1\end{array}$ & $\begin{array}{l}\text { 5'AAGAAGTCGGTGGACAAGAACAG'3 } \\
\text { 5'TGCGCACCGCGATGT'3 }\end{array}$ & 5'AACGAGTACCGGGTGCGGCG'3 \\
\hline $\begin{array}{l}\text { Lipoprotein lipase (LPL) } \\
\text { NM } 000237.2\end{array}$ & $\begin{array}{l}\text { 5'CAGCAAAACCTTCATGGTGAT'3 } \\
\text { 5'CAAGTTTTGGCACCCAACTC'3 }\end{array}$ & $5^{\prime} \mathrm{CATACATTCCTGTTACCGTCCAGCCA'3}^{\prime}$ \\
\hline $\begin{array}{l}\text { Fatty acid synthase (FAS) } \\
\text { NM 004104.4 }\end{array}$ & $\begin{array}{l}\text { 5'CACCTATGGCCTGCAGTGC'3 } \\
\text { 5'TGATGCAGTCGATGTAGTAGGCA'3 }\end{array}$ & 5'CCCGAGCTGCGCCCCTTG'3 \\
\hline $\begin{array}{l}\text { Fatty acid binding protein } 4 \text { (FABP- } \\
\text { 4) NM } 001442.2\end{array}$ & $\begin{array}{l}\text { 5'AAATGTGTGATGCTTTTGTAGGTACC'3 } \\
5 \text { 'CCATGCCAGCCACTTTCC' } 3\end{array}$ & $\begin{array}{l}\text { 5`ATGAAAGAAGTAGGAGTGGGCTTTGCCACC' } \\
3\end{array}$ \\
\hline $\begin{array}{l}\text { Glucose transporter } 4 \text { (GLUT-4) } \\
\text { NM 001042.2 }\end{array}$ & $\begin{array}{l}\text { 5'CCGCTACCTCTACATCATCCAGA'3 } \\
\text { 5'CAGAAACATCGGCCCAGC'3 }\end{array}$ & 5 'CCTGCCAGAAAGAGTCTGAAGCGCC'3 \\
\hline $\begin{array}{l}\text { Adipose triglyceride lipase (ATGL) } \\
\text { NM } 020376.3\end{array}$ & $\begin{array}{l}\text { 5'CGAGAATGTCATTATATCCCACTTCA'3 } \\
\text { 5'TGAGCCCACAGTACACGGG } 3\end{array}$ & 5'AAGGACGAGCTCATCCAGGCCAATG'3 \\
\hline $\begin{array}{l}\text { Hormone sensitive lipase (HSL/ } \\
\text { LIPE) NM } 005357.3\end{array}$ & $\begin{array}{l}\text { 5'CTGCATAAGGGATGCTTCTATC'3 } 3 \\
5 \text { 'CCTGTCTCGTTGCGTTTGTAGTA'3 }\end{array}$ & 5'CTGCCTGGGCTTCCAGTTCACGC'3 \\
\hline $\begin{array}{l}\text { Perilipin } 1 \text { (PLIN-1) } \\
\text { NM 001145311.1 }\end{array}$ & $\begin{array}{l}\text { 5'CCCCCTGAAAAGATTGCTTCT'3 } \\
5 \text { 'GGAACGCTGATGCTGTTTCTG'3 }\end{array}$ & 5'CCATCTCTCCACCCGCCTCCGC'3 \\
\hline Endogenous control & $\begin{array}{l}\text { Forward Primer } \\
\text { Reverse Primer }\end{array}$ & $\begin{array}{l}\text { Probe } \\
\text { (VIC conjugated) }\end{array}$ \\
\hline $\begin{array}{l}\text { 18S rRNA } \\
\text { NR } 003286.2\end{array}$ & $\begin{array}{l}\text { 5'CGGCTACCACATCCAAGG'3 } \\
\text { 5'CGGGTCGGGAGTGGGT'3 }\end{array}$ & 5'TTGCGCGCCTGCTGCCT’3 \\
\hline
\end{tabular}

\section{Supplemental Method S3.4: Detailed Statistical Analysis}

Statistical programming was performed with the statistical software $\mathrm{R}$ [1, version 3.0.2]. Additional packages used for analysis were multcomp [2, version 1.3-6] and nlme [3, version 3.1-117].

\section{Analysis of Array-Scan data}

For standardisation purposes, each 24-well plate contained a Diff CTRL (triplicates) and all treatment triplicates were calculated in $\%$ of the Diff CTRL average per plate.

All parameters were investigated for normality of distribution (visually and using the Shapiro Wilk test). A log-transformation (base 10) was performed to correct for observed skewness of the distribution of the data. Analysis then took place with a hierarchical linear mixed model. We included donor, treatment and donortreatment interaction as fixed effect and additionally allowed different variances per donor/treatment stratum, since visual inspection showed that variance heterogeneity between strata had remained even after log-transformation. To ensure that the assumptions of the used model were met we inspected goodness of fit plots.

The hierarchy of the experiment was reflected by using nested random effects (replicate nested in plate nested in passage number nested in experiment). Donor differences per treatment and overall and treatment differences (vs. Diff CTRL) per donor and averaged across donors were tested. p-values were adjusted for multiple comparisons for overall averages [2]. 


\section{Analysis of $\triangle \triangle C T$-values for gene expression data}

mRNA abundance was calculated using the comparative $\mathrm{C}_{\mathrm{T}}$ method: $\Delta \mathrm{C}_{\mathrm{T}}=\mathrm{C}_{\mathrm{T}}$ [gene of interest $]-\mathrm{C}_{\mathrm{T}}$ [endogenous control] and $\Delta \Delta \mathrm{C}_{\mathrm{T}}=\Delta \mathrm{C}_{\mathrm{T}}[$ Diff CTRL cells $]-\Delta \mathrm{C}_{\mathrm{T}}$ [treated cells]. $\Delta \Delta \mathrm{C}_{\mathrm{T}}$ values were calculated using the Diff CTRL cells per plate to standardize the data points. A hierarchical linear mixed model was used to estimate the mean $\Delta \Delta \mathrm{CT}$ across all experiments and donors. For this, we included treatment as fixed effect and additionally allowed different variances per donor/treatment stratum, since visual inspection showed variance heterogeneity between strata. The hierarchy of the experiment was reflected by using nested random effects (replicate nested in well nested in plate nested in donor). The estimated mean $\Delta \Delta \mathrm{CT}$ per treatment together with its estimated standard deviation were then used to calculate $2^{\wedge}(-\Delta \Delta C T)$ together with its range (+/- error based on SEM). To ensure that the assumptions of the used model were met we inspected goodness of fit plots.

\section{Analysis for adipokines in supernatants}

All parameters were investigated for normality of distribution (visually and by using the Shapiro Wilk test). A log-transformation (base 10) was performed to correct for observed skewness of the distribution of the data. Analysis then took place similar to before with a hierarchical linear mixed model. We included donor, treatment and donor-treatment interaction as fixed effect and additionally allowed different variances per donor/treatment stratum, since visual inspection showed that variance heterogeneity between strata had remained even after log-transformation.

The hierarchy of the experiment was reflected by using nested random effects (replicate nested in well nested in plate nested in experiment). Donor differences per treatment and overall and treatment differences (vs. Diff CTRL) per donor and averaged across donors were tested. Univariate and adjusted p-values were reported. To ensure that the assumptions of the used model were met we inspected goodness of fit plots.

\section{References}

1. R Core Team (2013). R: A language and environment for statistical computing. R Foundation for Statistical Computing, Vienna, Austria.

2. Torsten Hothorn, Frank Bretz and Peter Westfall (2008). Simultaneous Inference in General Parametric Models. Biometrical Journal 50(3), 346--363.

3. Pinheiro J, Bates D, DebRoy S, Sarkar D and R Core Team (2014). nlme: Linear and Nonlinear Mixed Effects Models. R package version 3.1-117 


\section{Supplemental Results}

Lipid Droplet Quantification (ArrayScan) - Day 14

A

120

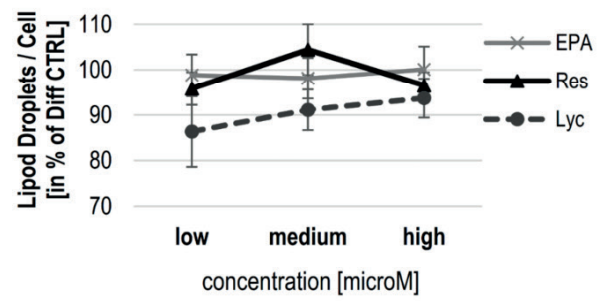

Gene Expression Analysis (TaqMan ${ }^{\mathrm{TM}}$ ) - Day 8

C

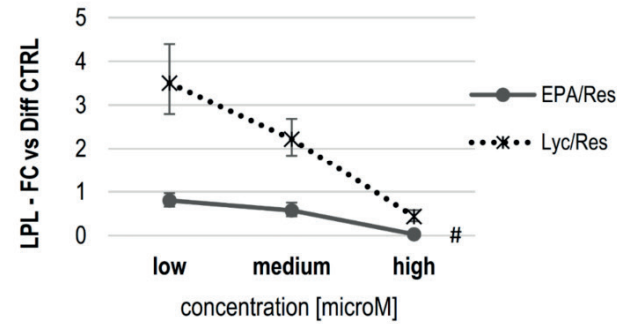

Adipo- and Cytokine Secretion (Luminex) - Day 8

E

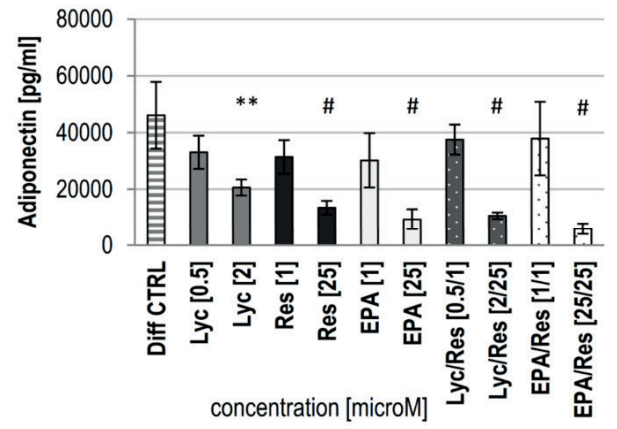

B

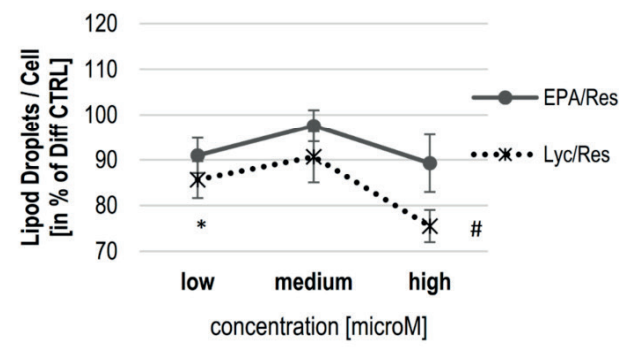

D

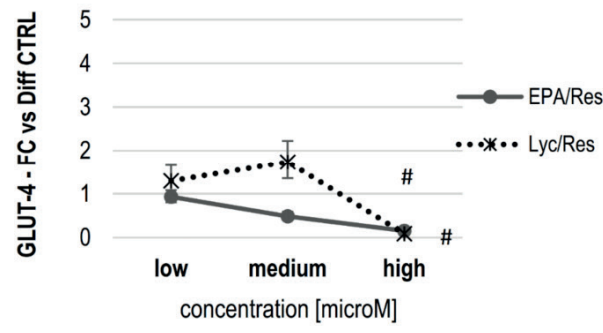

F

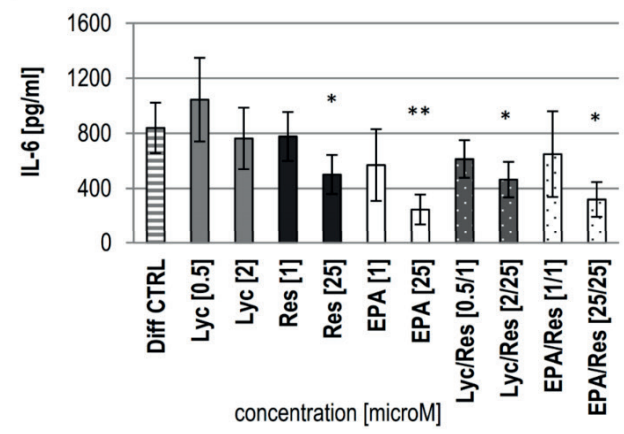

\section{Supplemental Figure S3.1: Lipid droplet number, lipogenic gene expression markers and adipokine secretion of adipocytes treated with different doses of bio-active compounds}

Assessment of different doses of Lyc, Res and EPA alone and of the combinations EPA/Res and Lyc/Res on distinct adipocyte parameters reflecting adipocyte function. Depicted are the effects on the number of lipid droplets (Lipid Droplets/Cell in \% of Diff CTRL; A-B), the expression of the lipogenic genes LPL and GLUT-4 (FC \pm error compared to Diff CTRL set as 1; C-D) and the secretion of the adipokines adiponectin and IL-6 (overall mean \pm SEM; E-F) after 8 (C-F) or 14 (A-B) days treatment of HPAd (donors $\geq 2, n \geq 2$ ). These data indicate that not all parameters follow a linear dose-response relationship. $\left(^{*}\right) p<0.05,\left({ }^{* *}\right) p<0.01$, (\#) $p<0.001$ (versus Diff CTRL, linear mixed model). 
Gene Expression Analysis (TaqMan ${ }^{\mathrm{TM}}$ ) - Day 14

A

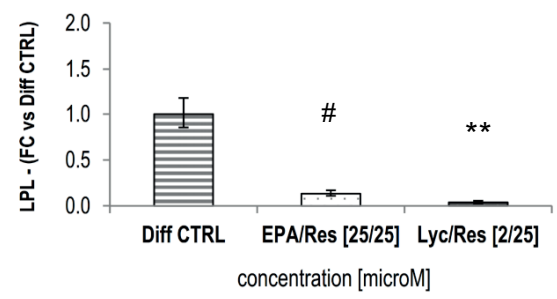

C

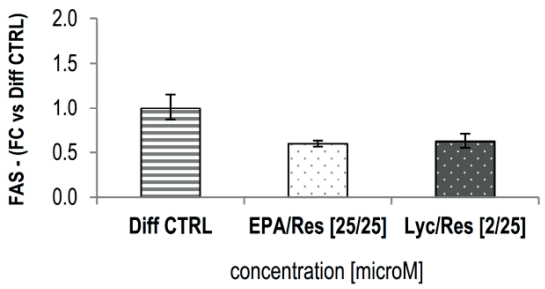

Adip- and Cytokine Secretion (Luminex) - Day 14

E

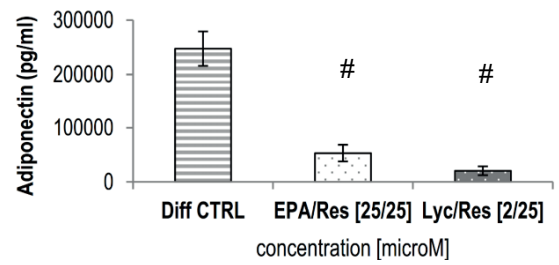

G

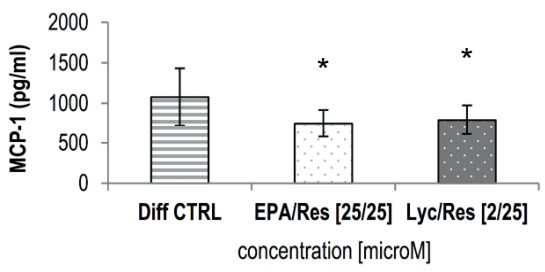

B

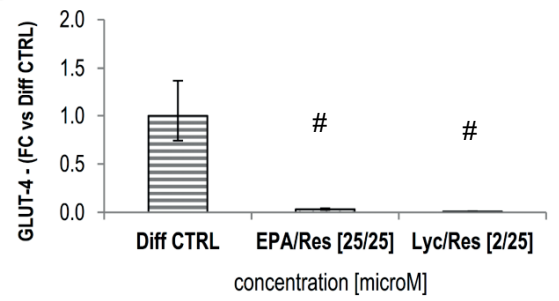

D

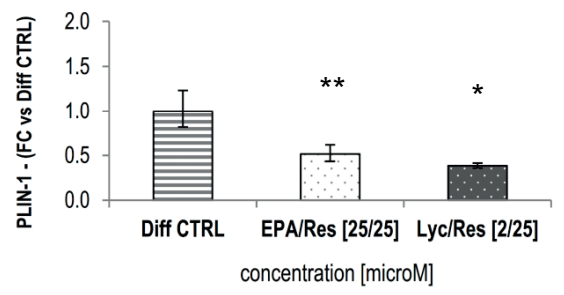

$\mathrm{F}$

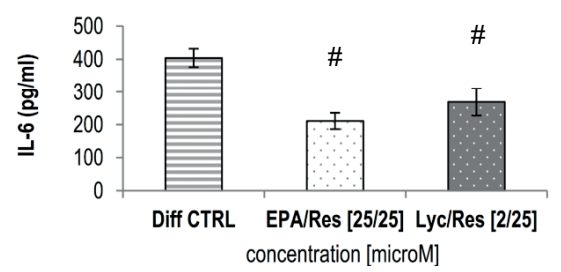

$\mathrm{H}$

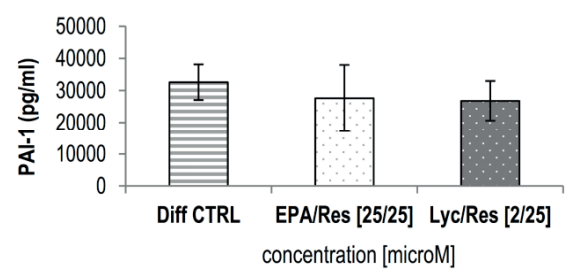

Supplemental Figure S3.2: Effects of bio-active compounds on lipogenic/lipolytic gene expression markers and adipokine secretion in differentiated human adipocytes

Shown are the effects of the combinations EPA/Res and Lyc/Res on gene expression and adipokine secretion after 14 days treatment of HPAd. mRNA levels of lipogenesis markers LPL (A), GLUT-4 (B) and FAS (C) and the lipolytic marker PLIN-1 (D) were determined by quantitative RT-PCR and the accumulation of the adipokines adiponectin (E), IL-6 (F), MCP-1 (G) and PAI-1 $(\mathrm{H})$ was measured in media after 5 days conditioning (day $10-14$ ) on the LiquiChip $§$ workstation. Data are shown as crude fold change $(F C) \pm$ error (based on SEM, A-D) compared to Diff CTRL set as 1 and as overall mean \pm SEM (E-H) for one experimental series (all donors included: HPAd 1375, 1377 and super lot SL0035). $\left(^{*}\right) p<0.05,\left({ }^{* *}\right) p<0.01,(\#) p<0.001$ (versus Diff CTRL, Student's t-test). 


\section{CHAPTER 4}

\section{Effects of combining resveratrol with lycopene on functional features of in-vitro differentiated human white adipocytes}

Ines Warnke, Joseph Schwager, Kenneth Verboven, Rotraut Schoop, Christine Toepfer, Ellen E. Blaak, Johan W.E. Jocken 


\section{Abstract}

Background: Combining dietary bio-active compounds is supposed to induce additive or synergistic effects on cell metabolism including adipocyte function, thereby improving metabolic profiles. These effects might depend on genetic and metabolic characteristics of the donor. Here we explored whether the combination of (all-E)-lycopene (Lyc) and trans-resveratrol (Res) influences functional features of in-vitro differentiated human multipotent adipose-derived stem (hMADS) cells from subcutaneous white adipose tissue of lean and obese donors.

Methods: hMADS cells were in-vitro differentiated in the presence or absence of Lyc $(2 \mu \mathrm{M})$ and Res $(25 \mu \mathrm{M})$ or their combination for 14 days. Subsequently, functional features of white adipocytes were determined including: i) intracellular lipid droplet (LD) accumulation (by fluorescent microscopy), ii) lipolytic capacity (by glycerol release), iii) adipocyte specific gene expression (via RT-PCR) and iv) adipokine secretion (with Luminex ${ }^{\circledR}$ technology).

Results: The combination Lyc/Res showed a strong inhibitory effect on LD accumulation $(\sim-59 \%)$ in differentiated hMADS cells independently of the donor's characteristics. However, Res alone affected the storage of LDs to a similar extent $(\sim-53 \%)$ as the combination with Lyc. In addition, Res and Lyc/Res blunted basal and beta-adrenergically stimulated lipolysis in differentiated hMADS cells by $72 \%$ and $50 \%$, and $64 \%$ and $48 \%$, respectively. These functional changes were accompanied by a decreased expression of the adipogenic (PPAR-gamma, CEBP-alpha), lipogenic (LPL, FABP-4, FAS, GLUT-4) and lipolytic genes (ATGL, HSL, PLIN-1). Furthermore, Res and the combination decreased the IL-6 and adiponectin secretion.

Conclusion: Collectively, our data suggest that mainly Res is the most potent bio-active compound which acts on adipocytes. Under the differentiation conditions utilized it improved adipocyte function independently of the donor's characteristics (lean versus obese). Therefore, Res may be beneficial for the prevention and treatment of metabolic disorders via amelioration of human white adipocyte function. 


\section{Background}

Adipose tissue (AT) is the major site for storing triacylglycerides (TAGs) in intracellular lipid droplets (LDs). Hence, it is the metabolic hub for energy storage and release depending on calorie in- and output. Adipocytes possess the enzymatic machinery necessary for the LDs life cycle, including lipoprotein lipase (LPL), involved in extracellular lipid hydrolysis releasing fatty acids (FA), which when taken up by the adipocytes are used to synthesize TAGs (lipogenesis). Contrary, adipose triglyceride lipase (ATGL) and hormone sensitive lipase (HSL) initiate the hydrolysis of intracellularly stored TAGs into free FAs and glycerol (lipolysis) [1]. Adipose LD turnover is regulated by a strong interplay between adipogenic differentiation, lipogenesis, and lipolysis. Consequently, AT dysfunction is characterized by an impaired adipogenesis, hypertrophic adipocytes, local inflammation, and disturbed lipid and glucose metabolism [2, 3]. Dysfunctional AT, rather than an increased fat mass per se, accompanies the highly prevalent condition obesity [4], affects whole-body insulin sensitivity [5] and is associated with the development of type 2 diabetes mellitus (T2DM) [6, 7]. Thus, comprehensive elucidation of mechanisms provoking and/or reducing AT dysfunction is the foundation for new treatment strategies to combat obesityassociated metabolic diseases.

Human AT cell models rely on the ability that stromal cells of AT can be differentiated in-vitro into multilocular adipocytes by stimulating the adipogenic transcriptional cascade (reviewed in [8]). The stromal vascular fraction (SVF), containing immune cells, stem cells and pre-adipocytes among other cells, actively contributes to the endocrine function of the AT organ [9]. AT of obese individuals often contains more immune cells including macrophages [10], which is associated with an increased secretion of pro-inflammatory cytokines and chemokines [11]. This local 'lowgrade inflammation' affects local adipocyte function, systemic inflammation, and insulin sensitivity [12].

Diets dense in nutritional bio-actives may have beneficial clinical effects on metabolic processes, among others by influencing adipocyte function. In recent years, numerous plant-derived polyphenols and carotenoids, or their metabolites have been investigated for their impact on adipocyte function in different murine models $[13,14,15]$. Several studies described beneficial effects for the polyphenol resveratrol (Res) on obesity-associated metabolic disorders [16]. Among these, studies using human pre-adipocyte cell lines have demonstrated that Res may be a potent modulator of adipocyte function (for review see [17]) by altering adipokine secretion [18, 19]. On the contrary, the carotenoid lycopene (Lyc) has been found to be an anti-inflammatory antioxidant, but effects on human adipocyte function are rarely evaluated and remain to be further investigated [20]. Recently, we have shown that combining trans-Res and (all-E)-Lyc improved adipocyte 
function in differentiated primary human pre-adipocytes (HPAd) by reducing LD accumulation, secretion of pro-inflammatory cytokines (IL-6 and MCP-1) and gene expression of lipogenic and lipolytic markers such as GLUT-4, LPL and the LD-covering protein perilipin (PLIN)-1 [18]. However, human data regarding the effects of bio-active compounds and combinations thereof on functional features of adipocytes are still scarce [21, 22, 23].

In the present study, we investigated whether the combination Lyc/Res influences adipocyte function of in-vitro differentiated human multipotent adipose-derived stem (hMADS) cells, also known as ASCs (adipose-derived stem cells). Since isolated hMADS cells appear to retain donor characteristics [24], we examined whether hMADS cells derived from subcutaneous AT of agematched obese and lean donors and purchased human pre-adipocytes (HPAd) differently respond to treatment with nutritional bio-actives. In-vitro adipocyte functional features were determined including: i) intracellular lipid droplet accumulation (by fluorescent microscopy), ii) basal and maximally beta-adrenergically stimulated lipolytic capacity (reflected by glycerol release), iii) adipocyte specific gene expression (via RT-PCR) and iv) adipokine secretion (using Luminex ${ }^{\circledR}$ technology).

\section{Material and Methods}

\section{Donors}

hMADS cells were isolated from individual abdominal subcutaneous white AT biopsies as described elsewhere $[25,26]$. Biopsies were collected from male subjects participating in a clinical research study, which was approved by the Medical Ethical Committee of Jessa Hospital in accordance with the Declaration of Helsinki (ClinicalTrial.gov accession number NCT02598544; [27]). Clinical characteristics of lean and obese donors used for the present study are summarized in Table 4.1. The lean group consisted of hMADS cells derived from 6 healthy donors [age 55+/2 years; BMI 23.2+/-0.4 kg/m²; glycated haemoglobin $\left.\left(\mathrm{HbA}_{1 \mathrm{c}}\right) 5.3+/-0.1 \%(34+/-1 \mathrm{mmol} / \mathrm{mol})\right]$. The obese group included hMADS cells of 5 donors [age $55+/-4$ years; BMI $37.8+/-0.96 \mathrm{~kg} / \mathrm{m}^{2}$; $\left.\mathrm{HbA}_{1 \mathrm{c}} 7.1+/-0.6 \%(55+/-7 \mathrm{mmol} / \mathrm{mol})\right]$. Three of the obese donors were diabetic $\left[\mathrm{HbA}_{1 \mathrm{c}} \geq 6.5 \%\right.$ (45 mmol/mol)].

\section{Reagents and cell culture}

All reagents and chemicals were purchased from Life Technologies (Karlsbad, CA, US) and from Sigma-Aldrich (St. Louis, MO, US), respectively, if not otherwise stated. 
In addition, human pre-adipocytes (HPAd) from subcutaneous white AT of female subjects (age 43.2 years, BMI 27.9; further details see [18]) from Cell Application and from Zen-Bio (two multidonor vials) were tested. Cells were cultured and differentiated as described previously [18].

Table 4.1: Donors characteristics (hMADS cells of subcutaneous abdominal AT)

\begin{tabular}{|c|c|c|c|c|c|c|}
\hline \multirow[b]{2}{*}{ Age (years) } & \multicolumn{3}{|c|}{$\begin{array}{l}\text { LEAN } \\
(n=6)\end{array}$} & \multicolumn{3}{|c|}{$\begin{array}{c}\text { OBESE } \\
(\mathrm{n}=5)\end{array}$} \\
\hline & 55.5 & \pm & 2.3 & 55.0 & \pm & 3.9 \\
\hline $\mathrm{BMI}\left(\mathrm{kg} / \mathrm{m}^{2}\right)$ & 23.2 & \pm & 0.4 & 37.8 ** & \pm & 1.0 \\
\hline $\mathrm{W}-\mathrm{H}$ ratio & 0.97 & \pm & 0.02 & $1.05 *$ & \pm & 0.02 \\
\hline LBM (kg) & 57.6 & \pm & 2.5 & $75.4^{* *}$ & \pm & 2.1 \\
\hline Fat mass $(\mathrm{kg})$ & 17.3 & \pm & 1.7 & $40.7^{* *}$ & \pm & 2.2 \\
\hline Body fat $\%$ & 23.1 & \pm & 2.1 & 35.0 ** & \pm & 1.6 \\
\hline Fasting glucose (mmol/L) & 5.7 & \pm & 0.2 & 8.7 & \pm & 1.3 \\
\hline Fasting insulin (mU/L) & 10.4 & \pm & 2.2 & $22.4^{*}$ & \pm & 2.7 \\
\hline Fasting triglycerides $(\mathrm{mmol} / \mathrm{L})$ & 0.96 & \pm & 0.14 & 1.14 & \pm & 0.3 \\
\hline Fasting FFA ( $\mu \mathrm{mol} / \mathrm{L})$ & 424.8 & \pm & 121.8 & 1097 * & \pm & 122 \\
\hline $\mathrm{HbA}_{1 \mathrm{c}}(\%)$ & 5.3 & \pm & 0.1 & $7.1^{*}$ & \pm & 0.6 \\
\hline HOMA-IR & 2.7 & \pm & 0.6 & 9.1 * & \pm & 2.3 \\
\hline
\end{tabular}

Values are means \pm SEM, subcutaneous abdominal biopsies, Caucasian males; for the three diabetic-obese subjects the HOMAIR was $11.5 \pm 3.4$ and the fasting insulin and glucose were $26 \pm 2.6 \mathrm{mU} / \mathrm{L}$ and $9.5 \pm 2.2 \mathrm{mmol} / \mathrm{L}$, respectively. BMl: body mass index, circ.: circumference, FFA: free fatty acids, HbA1c: glycated haemoglobin, HOMA-IR: homeostatic model assessment insulin resistance, LBM: lean body mass, SEM: standard error of the mean, W-H: waist-hip. * $p<0.05$; ${ }^{* *} p<0.001$ compared with lean control group.

Relevant adaptations of the protocol are briefly delineated. Growth medium (GM) was supplemented with or without $2.5 \mathrm{ng} / \mathrm{ml}$ recombinant basic fibroblast growth factor (bFGF), depending on the proliferation rate of the cells and was changed every 2-3 days. Cells were used between passage 4 and 8 . For experiments, 3000-6000 cells $/ \mathrm{cm}^{2}$ were grown on 24-well cell culture plates (Corning ${ }^{\circledR}$ Costar ${ }^{\circledR}$ TCT, one donor/plate) in GM until confluent. Cells were then stimulated to differentiate with differentiation medium (DM). Therefore DMEM/Ham's F-12 was supplemented with 3\% FCS, $17 \mu \mathrm{M}$ calcium-pantothenate, $33 \mu \mathrm{M}$ biotin, $0.1 \mu \mathrm{M}$ human insulin, $1 \mu \mathrm{M}$ dexamethasone (Dexa), $5 \mu \mathrm{M}$ rosiglitazone and $250 \mu \mathrm{M}$ isobutylmethylxanthine (IBMX), during the first 7 days. To complete adipocyte differentiation cells were maintained another 7 days (=7/7-day protocol) in DM omitting rosiglitazone and IBMX (maintenance medium, MM).

Non-differentiation control (CTRL) and differentiation control (Diff CTRL) cells were cultured in MM omitting Dexa and in DM/MM, respectively. For bio-actives treatment, media were supplemented with single test compounds (Res, $25 \mu \mathrm{M}$ and Lyc, $2 \mu \mathrm{M}$ (DSM Nutritional Products Ltd. Basel, Switzerland)) or a combination thereof for either the complete differentiation period 
(14 days) or 24 hours (day 14). Compound concentrations were based on dose-response experiments as presented in our previous study [18]. Lyc was dissolved in tetrahydrofurane (THF), Res in dimethylsulfoxide (DMSO) and controls and treatments were performed in triplicate or quadruplicate. Final DMSO and THF concentrations were adjusted for all treatments and controls to $0.2 \%$ and $0.1 \%$, respectively. Media and test compounds were replaced every 2 to 3 days.

\section{Measurement of lipid droplet accumulation in adipocytes}

Cellular LDs were quantified using an ArrayScan ${ }^{\circledR}$ VTI HCS Reader (equipped with a LED light source; ThermoFisher, Waltham, MA, US) by adapting our previously described high-content Cellomics $^{\circledR}$ assay [18] to the Colocalization ${ }^{\circledR}$ BioApplication V4. In brief, differentiated adipocytes (day 14, quadruplicates/treatment) were fixed with $60 \%$ isopropanol or $3.7 \%$ formaldehyde, stained with the fluorescent dyes Hoechst 33342 (final $4 \mu \mathrm{g} / \mathrm{ml}$ ) and BODIPY ${ }^{\circledR}$ 493/503 (final $0.125 \mu \mathrm{g} / \mathrm{ml}$ ) (Life Technologies) at RT for $30 \mathrm{~min}$, followed by image acquisition. Assay parameters are listed in Supplemental Table S4.1. For analysis 100 fields (objects) per well were scanned and data of four channels were used to report results on a "per nuclei" basis. The normalized LD features (number, area, and intensity) were calculated as percent of Diff CTRL per plate. Nuclei count and PrestoBlue ${ }^{\circledR}$ cell viability reagent (ThermoFisher) were used to assess cytotoxic effects of the compounds (day 14).

\section{In-vitro lipolysis in differentiated hMADS cells}

Differentiated hMADS adipocytes (14 days) were incubated in lipolysis medium (LM; DMEM /Ham's F-12 containing $3 \%$ bovine serum albumin (fatty acid-free), 1000 units/ml penicillin $/ 1000 \mu \mathrm{g} / \mathrm{ml}$ streptomycin, $0.25 \mu \mathrm{g} / \mathrm{ml}$ amphotericin B) supplemented with Res $(25 \mu \mathrm{M})$, Lyc $(2 \mu \mathrm{M})$, Lyc/Res $(2 / 25 \mu \mathrm{M})$ or only DMSO/THF $(0.2 \% / 0.1 \%$, vehicle CTRL) overnight. Next day, cells were treated with bio-actives in either the presence or absence of $1 \mu \mathrm{M}$ isoprenaline (ISO, a non-selective $\beta$-adrenergic agonist) for 5 hours. After the 5 -hour incubation period, aliquots of the supernatants were collected and analyzed in duplicates for glycerol concentrations (EnzyChrom ${ }^{\mathrm{TM}}$ Adipolysis Assay Kit; CAT\#EAPL-200; Gentaur). Treatments were performed in triplicates and data are expressed as normalized mean glycerol release ( $\mu \mathrm{g} / \mathrm{mg}$ protein). For protein quantification, cells were washed with ice-cold PBS, lysed in $40 \mu \mathrm{l}$ RIPA (radioimmunoprecipitation assay) buffer containing 1x protease and phosphatase inhibitor cocktail (Cell Signaling Technology) and stored at $-80^{\circ} \mathrm{C}$ prior to analysis. Protein measurements were performed in duplicates using the Pierce ${ }^{\circledR}$ BCA protein assay kit (Santa Cruz Biotechnology). 


\section{RNA extraction and quantification by RT-PCR (gene expression analysis)}

Total RNA was collected and isolated at day 0, 7 and 14 of treatment (Figure 4.1A) using RNeasy ${ }^{\circledR}$ 96 Kits (Qiagen, Hilden, Germany). Primers and probes were designed and synthesised as described previously [18]. Quantitative TaqMan ${ }^{\mathrm{TM}}$ real-time (RT) PCR was performed on first strand cDNA (Omniscript ${ }^{\circledR}$ RT Kit; Qiagen) transcribed from 100-500 ng RNA [28] utilizing an ABI-PRISM ${ }^{\circledR} 7900$ HT Sequence Detection System. mRNA abundance was calculated using the comparative $\mathrm{C}_{\mathrm{T}}$ method: $\Delta \mathrm{C}_{\mathrm{T}}=\mathrm{C}_{\mathrm{T}}$ [gene of interest $]-\mathrm{C}_{\mathrm{T}}$ [endogenous control] and $\Delta \Delta \mathrm{C}_{\mathrm{T}}=\Delta \mathrm{C}_{\mathrm{T}}$ [Diff CTRL cells] $-\Delta \mathrm{C}_{\mathrm{T}}[$ treated cells $]$. The fold change for the gene of interest was expressed as $2^{-\Delta \Delta \mathrm{C}_{\mathrm{T}}}$.

\section{Analysis of secreted proteins from treated adipocytes}

Cell culture supernatants of differentiated adipocytes were collected, during and at the end of the treatment period (Fig. 1A), pooled and stored at $-80^{\circ} \mathrm{C}$ until further quantification. Therefore, 6plex Human Obesity Premixed kits (adiponectin/Acrp30, adipsin/complement factor D, IL-6, MCP-1/CCL-2, PAI-1/Serpin E1 and TNF-alpha; Luminex ${ }^{\circledR}$ Performance Assay: Cat\#FCST0806) were purchased from R\&D Systems (Minneapolis, Minnesota, USA) and used according to the Manufacture's protocol on the LiquiChip Workstation IS 200 (Luminex technology; Qiagen, Hilden, Germany). Pooled samples were measured in duplicates and final concentrations were normalized by the average nucleus count over four wells. Data were evaluated with the LiquiChip Analyser software from Qiagen.

\section{Statistical analysis}

Analyses were performed with the statistical software Minitab ${ }^{\circledR}$ 17.1.0 for Microsoft Windows. For standardisation purposes of the ArrayScan ${ }^{\circledR}$ data over repetitive experiments each 24-well plate contained a Diff CTRL (quadruplicates) and all treatment quadruplicates were calculated in $\%$ of the Diff CTRL average ( $=100 \%)$ and averaged across technical replicates per plate (donor). hMADS cells that did not differentiate properly (Window of LD number [Diff CTRL/CTRL] $<2$ ) were excluded from all analyses. Lipolysis data were normalized by protein content and are expressed relative to the corresponding vehicle CTRL average $(=100 \%)$ for each donor. Gene expression data are expressed as fold change (FC) \pm error (based on SEM) calculated from fitted $\Delta \Delta \mathrm{C}_{\mathrm{T}}$ means, whereas statistical analysis was performed with the averaged $\Delta \mathrm{C}_{\mathrm{T}}$ and $\Delta \Delta \mathrm{C}_{\mathrm{T}}$ values. Cyto-/adipokine concentrations are shown as absolute crude mean \pm SEM normalized by average cell number. For all parameters, visually inspected data were investigated for normality of distribution using the Ryan-Joiner (like Shapiro Wilk) and Kolmogorov-Smirnov test. A logtransformation (base 10) was performed to correct for the skewness of distribution of the data. If data were normally distributed, statistical significance of the mean differences between treatments 
was tested by analysis of variance (ANOVA) followed by Tukey's post hoc test (pairwise comparison adjusted for multiple testing). We included time point of experimental series (block), group (lean, obese, purchased) and treatment as fixed factors. Not normally distributed log transformed data were in addition analysed with the non-parametric Kruskal-Wallis test. The level of statistical significance was set as 0.05 .

\section{Results}

Overall means were calculated for all determined parameters because Diff CTRL cells of the groups (lean, obese, purchased) and the treatment effects were not significantly different between donor groups (Supplemental Figure S4.1). The treatments did not affect cell viability $(>70 \%$ compared to CTRL).

\section{Res and combined treatment reduced lipid droplet accumulation in adipocytes}

First, we investigated the chronic effects of bio-active compounds on LD accumulation in differentiated human adipocytes (day 14, Figure 4.1A) by automated fluorescent microscopy.

Res alone $(25 \mu \mathrm{M})$ significantly reduced the LD-number, -area and -intensity by $42 \%, 63 \%$ and $54 \%$, respectively, compared to Diff CTRL $(\mathrm{p}<0.001)$, whereas Lyc alone $(2 \mu \mathrm{M})$ did not affect LD accumulation (Figure 4.1B). The combination Lyc/Res $(2 / 25 \mu \mathrm{M})$ also reduced the adipocyte lipid content compared to Diff CTRL $(\mathrm{p}<0.001)$, but this was not significantly different in comparison to Res alone. Together, these data indicate that Res and Lyc/Res reduced LD accumulation to a similar extend in human adipocytes (Figure 4.1B) and this seems independent of donor characteristics (Supplemental Figure S4.1A). 
A

Growth Medium (GM)
Differentiation Medium (DM)

\section{4 days}

\section{Maintenance medium (MM)}

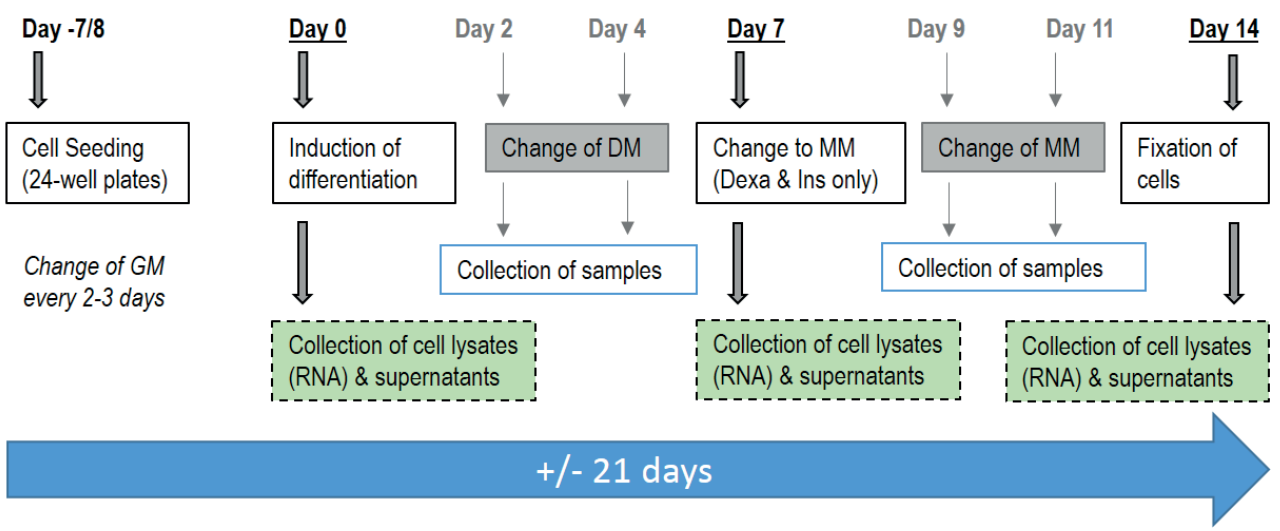

B

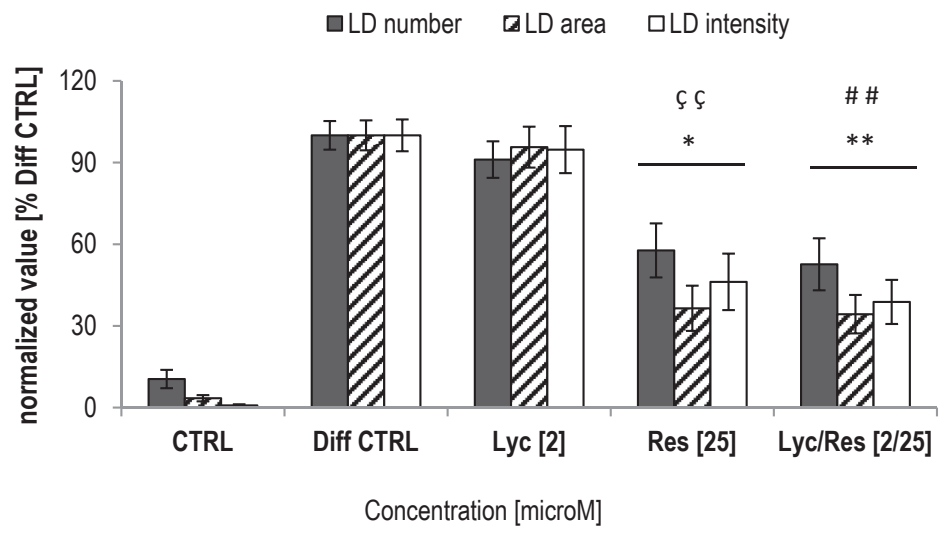

Figure 4.1: Lipid accumulation in differentiated human adipocytes treated in-vitro with lycopene (Lyc) and resveratrol (Res)

Primary hMADS cells from lean $(n=6)$ and obese $(n=3)$ donors and purchased HPAd $(n=4)$ were differentiated for 14 days in the presence of Lyc and Res alone or the combination thereof. Experimental time line (A). Effects of Lyc $(2 \mu \mathrm{M})$ and $\operatorname{Res}(25 \mu \mathrm{M})$ alone, and the combination Lyc/Res $(2 / 25 \mu \mathrm{M})$ on lipid droplet formation (B). Shown are three parameters (LD number, LD area and LD intensity; normalized by nuclei number) that quantify lipid accumulation in adipocytes, as compared to differentiation-only medium (Diff CTRL) set as 100\%. Data are represented as overall mean \pm SEM ( $n \geq 13$ donors, technical quadruplicates / treatment). CTRL: control; Dexa: dexamethasone; Diff: differentiation; hMADS: human multipotent adipose-derived stem cells; HPAd: human preadipocytes; Ins: insulin; LD: lipid droplet; Lyc: lycopene; Res: resveratrol; SEM: standard error of the mean. Statistical significance was determined by ANOVA (General Linear Model with Tukey's post hoc test - pairwise comparison adjusted for multiple testing). * Bio-actives alone or in combination compared to Diff CTRL $\left(^{*}\right) p<0.05$, $\left({ }^{*} *\right) p<0.001$ / \# Bio-active combination compared to Lyc [2] (\#) $p<0.05$, (\#\#) $p<0.001 /+$ Bio-active combination compared to Res [25] (+) $p<0.05,(++) p<0.001 /$ Ç Res [25] compared to Lyc [2] (ç) $p<0.05,(c ̧ \varsigma) p<0.001$ 


\section{Attenuated lipolysis after treatment of adipocytes with bio-actives}

In order to understand how dietary bio-actives might influence LD accumulation in human adipocytes, we measured the acute effect on basal and ISO-mediated lipolysis in 14-day differentiated hMADS cells. Res strongly inhibited basal (by $72 \%$, Figure $4.2 \mathrm{~A}$ ) and maximal ISOmediated lipolysis (by 50\%, Figure 4.2B) after the 5-hour treatment compared to vehicle CTRL $(p<0.05)$ and Lyc treatment $(p<0.001)$. Release of glycerol was also significantly reduced by the combination Lyc/Res, under basal (by 64\%) and ISO-mediated conditions (by 48\%, Figure 4.2, $\mathrm{p}<0.05$ ), but was not significantly different from Res alone. However, Lyc alone did not affect lipolysis in these adipocytes. There was no difference in basal and maximal ISO-mediated lipolysis (fold change: ISO-stimulated over basal) between the lean and obese group (data not shown). Overall, these findings indicate that the reduction in basal and beta-adrenergic stimulated lipolytic response was mainly mediated by Res.

\section{Combining Res and Lyc reduced gene expression of adipocyte markers}

To evaluate the ability of Lyc and Res to regulate transcriptional control of genes underlying the decreased LD accumulation and blunted lipolysis, a detailed analysis of adipogenic, lipogenic or lipolytic gene expression was conducted at day 14 of differentiation. Lyc alone $(2 \mu \mathrm{M})$ did not influence adipogenic, lipogenic or lipolytic gene expression (Figure 4.3A-D). However, Res $(25 \mu \mathrm{M})$ and the combination Lyc/Res $(2 / 25 \mu \mathrm{M})$ attenuated the expression of the adipogenic master regulators PPAR-gamma and C/EBP-alpha up to 0.36 -fold and 0.26 -fold $(\mathrm{p}<0.05)$, respectively (Figure 4.3A).

mRNA levels of the lipogenic genes FAS (fatty acid synthase), GLUT-4 (glucose transporter type 4/SLC2A4, (0.01-fold, $\mathrm{p}<0.001)$ ), FABP-4 (cytosolic fatty acid binding protein 4) and LPL (lipoprotein lipase) were also significantly reduced by Res alone and the combination compared to Diff CTRL (Figure 4.3B+C). In line with the decreased lipolytic response, Res and Lyc/Res strongly blunted the mRNA expression of HSL $(<0.05$-fold, Figure 4.3D) compared to Lyc $(p<0.001)$. The mRNA levels of ATGL and PLIN-1 (Figure 4.3D) were also significantly reduced by Res and Lyc/Res $(\mathrm{p}<0.05)$. However, combining the two bio-active compounds did not lead to a considerably larger reduction in gene expression than by Res alone $(p>0.26)$. These data corroborate that the modulation of the adipogenic, lipogenic and lipolytic gene expression in differentiated HPAds and hMADS cells (Supplemental Figure S4.2C-F) is mainly ascribable to Res and seems unaffected by the metabolic characteristics of the donor (Supplemental Figure S4.1C-D). 
A

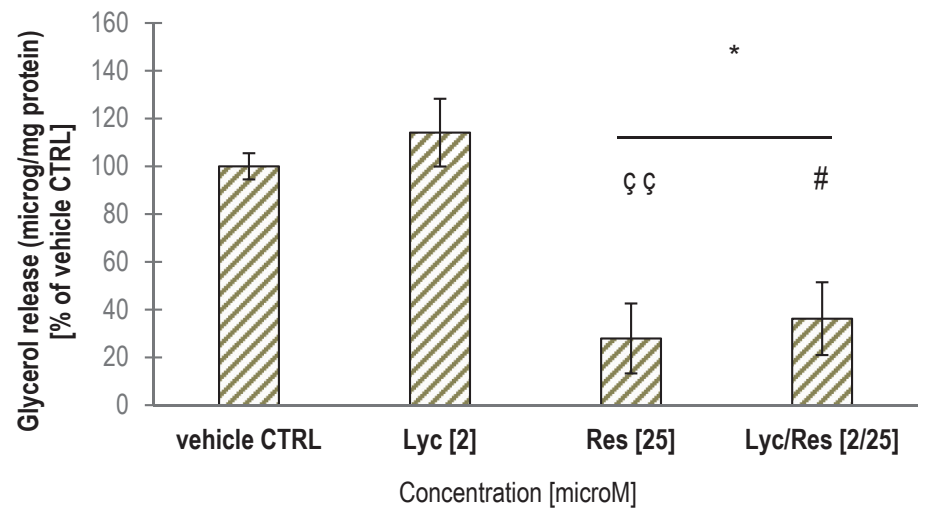

B

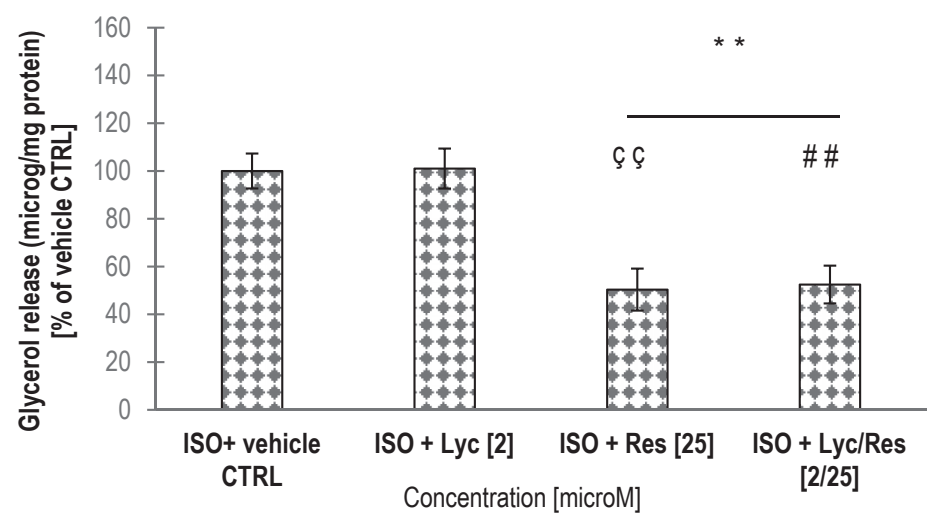

Figure 4.2: Decreased lipolysis in differentiated human adipocytes after in-vitro treatment with Lyc and Res

Glycerol release ( $\mu \mathrm{g} / \mathrm{mg}$ protein) was determined in 14 days differentiated hMADS cells under basal conditions (per $5 \mathrm{~h}$ incubation with bio-actives) (A) and ISO-mediated conditions ( $5 \mathrm{~h}$ incubation with bio-actives in the presence of ISO $\left(10^{-6} \mathrm{M}\right)$ ) (B). Cells were incubated with $2 \mu \mathrm{M}$ Lyc, $25 \mu \mathrm{M}$ Res or the combination thereof $(2 / 25 \mu \mathrm{M}$ Lyc/Res) for $18 \mathrm{~h}$ prior to the experiment. Data were normalized by protein amount, and results are expressed as means $\pm S E M(n=6 ; 3 x$ lean and $3 x$ obese donors, technical triplicates / treatment). Vehicle: DMSO/THF (0.2\%/0.1\%); CTRL: control; hMADS: human multipotent adiposederived stem cells; Lyc: Iycopene; Res: resveratrol; SEM: standard error of the mean; ISO: isoprenaline. Statistical significance was determined by ANOVA (General Linear Model with Tukey's post hoc test - pairwise comparison adjusted for multiple testing). * Bio-actives alone or in combination compared to vehicle CTRL $\left(^{*}\right) p<0.05,\left(^{*} *\right) p<0.001$ / \# Bio-active combination compared to Lyc [2] (\#) $p<0.05$, (\# \#) $p<0.001 /$ + Bio-active combination compared to Res [25] (+) $p<0.05,(++) p<0.001 /$ Ç Res [25] compared to Lyc [2] (६) $p<0.05$, (६ c) $p<0.001$ 
A

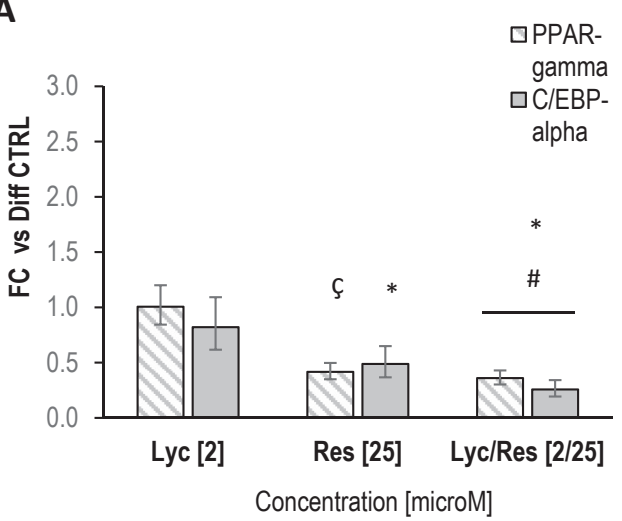

C

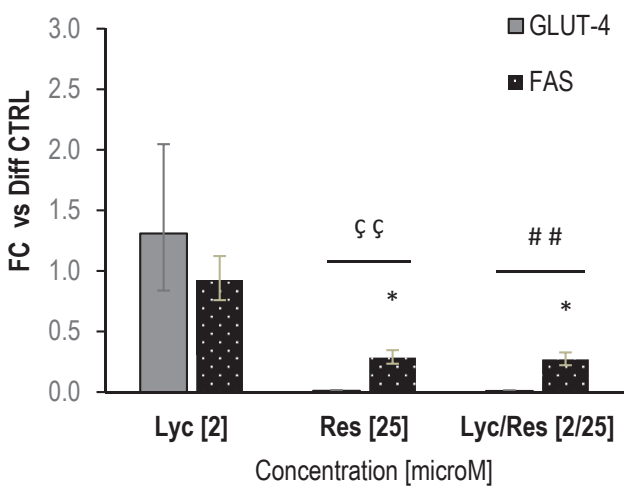

B

曰LPL

$\square$ FABP-4

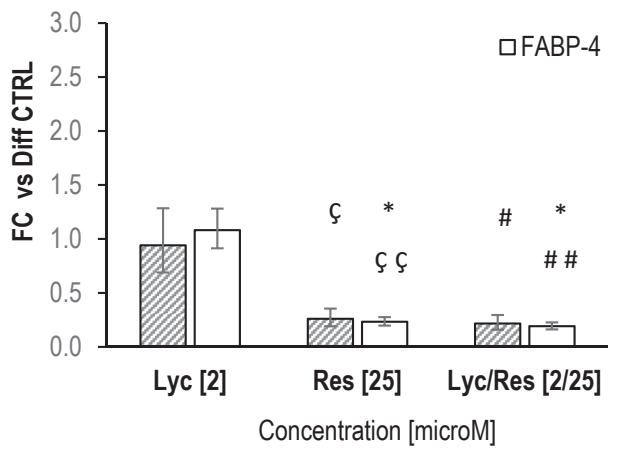

D

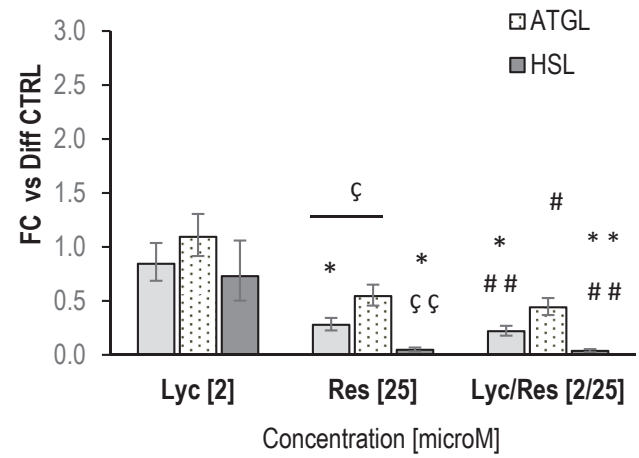

Figure 4.3: Adipogenic and lipogenic gene expression of human adipocytes treated in-vitro with Lyc and Res

Primary hMADS cells from lean $(n=6)$ and obese $(n=4)$ donors and purchased HPAd $(n=4)$ were differentiated for 14 days in the presence of Lyc and Res alone or the combination thereof. The effect on gene expression was determined by quantitative RT-PCR at day 14, including the mRNA levels of adipogenic transcription factors PPAR-gamma and C/EBP-alpha (A), lipogenesis markers LPL, FABP-4 (B), GLUT-4 and FAS (C) and lipolysis associated proteins PLIN-1, HSL and ATGL (D). Data are expressed as FC \pm error (based on SEM) over Diff CTRL (Diff CTRL set as 1 , not shown; $n \geq 13$ donors, technical quadruplicates / treatment). CTRL: control; Diff: differentiation; FC: fold change; hMADS: human multipotent adipose-derived stem cells; Lyc: lycopene; Res: resveratrol; SEM: standard error of the mean. Genes: ATGL: Adipose triglyceride lipase; C/EBP-alpha: CCAAT/enhancer binding protein alpha; FABP4: Fatty acid binding protein 4, FAS: Fatty acid synthase, GLUT-4: Glucose transporter 4; HSL: Hormone sensitive lipase; LPL: lipoprotein lipase; PLIN-1: Perilipin 1; PPAR-gamma: peroxisome proliferator-activated receptor gamma. Statistical significance was determined by ANOVA (General Linear Model with Tukey's post hoc test - pairwise comparison adjusted for multiple testing).

${ }^{*}$ Bio-actives alone or in combination compared to Diff CTRL $\left.\left(^{*}\right) p<0.05,{ }^{* *}\right) p<0.001$ / \# Bio-active combination compared to Lyc [2] (\#) $p<0.05$, (\#\#) $p<0.001 /$ + Bio-active combination compared to Res [25] (+) $p<0.05,(++) p<0.001 /$ Ç Res [25] compared to Lyc [2] (६) $p<0.05$, (ç ६) $p<0.001$ 


\section{Lyc/Res decreased pro-inflammatory secretion of differentiated adipocytes}

Finally, we determined the effects of dietary bio-actives on cyto-/adipokine secretion in in-vitro differentiated HPAd and hMADS adipocytes. After the 14-day treatment, Res alone and Lyc/Res significantly reduced adiponectin concentration $(\sim 80 \%)$ in the supernatants compared to Diff CTRL (Table 4.2, p<0.001). Concomitantly, Res and Lyc/Res reduced the level of IL-6 by $\sim 55 \%$ $(\mathrm{p}<0.05)$. The concentrations of MCP-1, TNF-alpha, PAI-1 and adipsin were not affected by any treatment (Table 4.2). A similar reduction in adiponectin and IL-6 concentrations was observed on day 2, 7, and 9 of differentiation (data not shown). Together, our data indicate that Res seems to be the effective bio-active that attenuates the secretion of the pro-inflammatory IL-6, and simultaneously suppressed the anti-inflammatory and insulin-sensitizing adiponectin.

Table 4.2: Decreased adipokine secretion in supernatants of differentiated primary adipocytes treated in-vitro with Lyc and Res for 14 days

\begin{tabular}{|c|c|c|c|c|c|c|c|c|c|c|c|c|c|c|}
\hline \multirow{2}{*}{$\begin{array}{l}\begin{array}{l}\text { Treatment } \\
\text { [microM] }\end{array} \\
\text { Adipokine }\end{array}$} & \multicolumn{2}{|c|}{ Diff CTRL } & \multicolumn{3}{|c|}{ Lyc [2] } & \multicolumn{3}{|c|}{ Res [25] } & \multicolumn{3}{|c|}{ Lyc/Res [2/25] } & \multicolumn{3}{|c|}{$\begin{array}{l}\text { Tukey's post hoc } \\
\text { Tests * }\end{array}$} \\
\hline & Mean & SEM & Mean & SEM & $p^{\#}$ & Mean & SEM & $p^{\#}$ & Mean & SEM & $p^{\#}$ & $\begin{array}{l}\text { Lycl } \\
\text { Res - } \\
\text { Lyc } \\
\end{array}$ & $\begin{array}{l}\text { Res - } \\
\text { Lyc }\end{array}$ & \begin{tabular}{|l|} 
Res - \\
Lycl \\
Res \\
\end{tabular} \\
\hline Adiponectin & 234647 & 27272 & 221810 & 26763 & 0.99 & 44731 & 26763 & 0.00 & 42848 & 27272 & 0.00 & 0.00 & 0.00 & 1.00 \\
\hline Adipsin & 230262 & 37827 & 207649 & 37124 & 0.97 & 189982 & 37124 & 0.87 & 199675 & 37124 & 0.94 & 0.99 & 0.99 & 0.99 \\
\hline IL-6 & 3139 & 401 & 1940 & 401 & 0.15 & 1336 & 401 & 0.01 & 1467 & 409 & 0.02 & 0.84 & 0.04 & 0.99 \\
\hline MCP-1 & 2963 & 389 & 3038 & 389 & 0.99 & 2900 & 389 & 0.99 & 3124 & 389 & 0.99 & 0.99 & 0.99 & 0.98 \\
\hline PAI-1 & 173140 & 39462 & 199161 & 38729 & 0.97 & 209965 & 38729 & 0.91 & 246192 & 38729 & 0.55 & 0.82 & 0.99 & 0.91 \\
\hline TNF-alpha & 462 & 96 & 434 & 95 & 0.98 & 570 & 94 & 0.85 & 587 & 96 & 0.78 & 0.66 & 0.74 & 0.99 \\
\hline
\end{tabular}

By cell number normalized values shown for three independent experimental series as overall mean \pm SEM $(n=14)$. Diff CTRL: Differentiation control; Lyc: lycopene; Res: resveratrol; SEM: standard error of the mean. $p$ \# - $p$-value compared to Diff CTRL, * Adjusted p-value (ANOVA - General Linear Model with Tukey's post hoc test, simultaneous tests for differences of means)

\section{Discussion}

Healthy adipocytes display functional features, which are essential for the physiological role of adipose tissue as a lipid storing and active endocrine organ. In the present study, we investigated whether the two regularly consumed bio-actives Res and Lyc, or both components combined, influence adipocyte function of in-vitro differentiated human adipocytes. Res and its combination with Lyc (Lyc/Res) demonstrated a significant reduction in intracellular LD accumulation, unaffected by the metabolic characteristics of the donor. This reduced LD accumulation was accompanied by a suppressed basal and beta-adrenergically mediated lipolysis, decreased expression of adipogenic, lipogenic and lipolytic markers, and an attenuated secretion of the proinflammatory cytokine IL-6 and the adipocyte-specific-hormone adiponectin. Overall, our data 
clearly indicate that Res is the main driver for these improved functional features of white adipocytes under the utilized differentiation conditions, independent of donor characteristics.

It is well known, that bio-active compounds contained in diets can influence adipogenesis and adipocyte function $[15,16,29]$. In our study, Res $(25 \mu \mathrm{M})$ alone and Lyc/Res $(2 / 25 \mu \mathrm{M})$ treatment showed significant effects on the LD accumulation in differentiated human adipocytes derived from white AT (Figure 4.1B). This is in line with the general reported findings of in-vitro and animal studies, which suggest that Res suppresses lipid storage and adipogenesis in rodent adipocytes [30, 31, 32]. In human Simpson-Golabi-Behmel syndrome (SGBS) adipocytes, previous studies reported anti-adipogenic and anti-lipogenic effects of Res for concentrations $>30 \mu \mathrm{M}[33,34]$. In contrast, enhancing effects on murine 3T3-L1 cells differentiation were also described for Res at low doses (1-10 $\mu \mathrm{M})$ [35]. However, in our recent study LD accumulation was unaffected in primary HPAd by $25 \mu \mathrm{M}$ Res [18]. The current observation implies that Res at $25 \mu \mathrm{M}$ seems only anti-adipogenic when the PPAR-gamma agonist rosiglitazone is not contained in the adipogenic cocktail for the complete 14-day differentiation period. This suggests an interaction between the polyphenol Res and this insulin-sensitizing PPARgamma agonist, corroborating our previous hypothesis that $25 \mu \mathrm{M}$ Res is insufficient to counteract the adipogenic effects of $100 \mu \mathrm{M}$ rosiglitazone when constantly present in the culturing environment [18]. Furthermore, in the present study, Res reduced the mRNA levels of both master regulators of adipogenesis, PPAR-gamma and C/EBP-alpha, in differentiated human adipocytes. This is in accordance with the reported down-regulation of PPAR-gamma, C/EBP-alpha and other lipogenic markers in 3T3-L1 [36] and SGBS adipocytes [33] at concentrations $\geq 20 \mu \mathrm{M}$ and the accompanying reduction of lipid accumulation in these cells and in fat pads of mice after a 10week high fat diet (HFD) supplemented with $0.4 \%$ Res [37]. In addition, GLUT-4, LPL, FAS and FABP-4, all markers of lipogenesis, were significantly down-regulated by Res and Lyc/Res (Fig. 4.3), which reflects previously reported data for Lyc/Res in maturing adipocytes but not for Res alone [18]. These discrepant effects of Res on e.g. PPAR-gamma, LPL and FABP-4 mRNA levels could partly be explained by the absence of rosiglitazone in the maintenance medium, as already indicated above. In this respect, in 3T3-L1 and SGBS adipocytes reduced mRNA levels of GLUT4 [38], FAS [33] and FABP-4 [35] were also shown after Res (>20-50 $\mu \mathrm{M})$ treatment. Together, the substantial down-regulation of the adipogenic and lipogenic gene expression and the comparable reduction in LDs by Res and Lyc/Res are indications that Res is the main driver for the reduction in adipogenic differentiation.

Human clinical trials with Res supplementation are scarce and showed controversial results regarding its effects on adipocyte size, AT function and inflammation [39-41]. However, it has 
been argued that Res is beneficial with respect to improving insulin sensitivity and overall metabolic health in subjects with T2DM [42, 43]. Conversely, recent data by Timmers et al. [44] and Most et al. [45] demonstrated no insulin-sensitizing effect of Res or Res in combination with epigallocatechin-3-gallate (EGCG) in T2DM and overweight-obese subjects, respectively, but Timmers et al. reported a substantial interaction between metformin (glucose-lowering medication) intake and Res metabolism [44]. Overall, the current in-vitro results and recent human data support the hypothesis of an interaction between drugs ameliorating the glucose homeostasis and the PPAR-targeting polyphenol, which needs to be investigated further.

The balance between adipogenesis, lipogenesis and lipolysis largely determines total lipid storage in human adipocytes. Lipolysis leads to a release of glycerol and free FAs from TAGs, and can be stimulated by several lipolytic agents such as isoprenaline, a non-selective beta-adrenoceptor agonist. The treatment with Res and Lyc/Res blunted basal and ISO-mediated lipolysis (Figure 4.2). However, for Res contradictory effects on adipocyte lipolysis have been reported for in-vitro and ex-vivo studies [46-50]. Most of these recent studies described either no effect on basal glycerol release [47, 50] or an increase of basal lipolytic activity [34, 48, 49] by Res, using varying concentrations $(0.03 \mu \mathrm{M}-1 \mathrm{mM})$, cell models (isolated rat, piglet and human white adipocytes, mature SGBS and 3T3-L1 fat cells) and incubation times (1.5 - $48 \mathrm{~h})$. In human primary [47], SGBS and murine 3T3-L1 adipocytes [49], an enhancement of ISO-stimulated lipolysis was described when treated with 10 or $100 \mu \mathrm{M}$ Res for 4 or $24 \mathrm{~h}$, respectively. A similar effect of Res $(100 \mu \mathrm{M})$ was also reported for epinephrine-stimulated lipolysis in rat adipocytes [50]. Our observations regarding the suppression of the basal and ISO-mediated glycerol release by Res $(25 \mu \mathrm{M})$ are in line with the findings from Chang et al. [46], who reported that Res at lower concentrations $(0.03-10 \mu \mathrm{M}, 24 \mathrm{~h})$ blunted basal and TNF-alpha-stimulated glycerol release in murine 3T3-L1 adipocytes. To our knowledge, for Lyc no effects on adipocyte lipolysis are described, which was confirmed in the current study because Lyc alone or combining Lyc with Res did not modify the anti-lipolytic effect of Res alone. In contrast to previous reports [35, 48], we show that chronic treatment with Res and Lyc/Res during the differentiation significantly reduced the gene expression of the lipolytic markers ATGL and PLIN-1. In the study by Lasa et al. treatment of mature human SGBS and murine adipocytes with Res (100 $\mu \mathrm{M}, 24 \mathrm{~h})$ [49] showed no effect on HSL gene expression, which was highly suppressed in our human adipocytes after 14 days treatment. However, it needs to be corroborated whether these gene expression changes in adipocytes translate into functional changes in lipolysis. Supplementing Res can have beneficial health effects in overweight and obese subjects, as partial inhibition of adipocyte lipolysis (HSL) could minimize lipid overflow and ectopic lipid accumulation in other tissues and thereby 
preventing the development of insulin resistance [17, 51]. It is noteworthy to mention that human clinical trials testing Res (30 days, $150 \mathrm{mg} / \mathrm{d})$ [40, 52] or a combination of Res and EGCG (12 weeks, 80 and $282 \mathrm{mg} / \mathrm{d}$ ) [45] have reported positive influences on certain AT functions and metabolic health in overweight-obese subjects.

Lyc is one of the most abundant carotenoid in plasma and is well studied for its anti-oxidative and anti-inflammatory effects in humans $[53,54]$. Our results suggest that Lyc $(2 \mu \mathrm{M})$ alone did not exhibit anti-lipogenic or anti-inflammatory effects in differentiated human adipocytes (Figure 4.1B). Contrary, moderate inhibitory effects were previously described by our lab in murine [28] and human adipocytes [18], which could partly be explained by the use of an alternative differentiation protocol. However, Lyc or its metabolite apo-10'-lycopenoic acid (APO10LA) did not modulate adipogenesis in 3T3-L1 adipocytes [55] or AT mass in 6 weeks supplemented rats [56], either. Furthermore, some controversial results exist regarding Lyc's impact on adipose gene expression. In accordance with our reported human adipocyte data, Lyc treatment did not affect adipogenic genes [55] but it moderately increased GLUT-4 and LPL mRNA expression, accompanied by a decreased lipid deposition, in murine adipocytes [28], and decreased PPARgamma mRNA in AT of rats without changes in total fat mass after 6 weeks Lyc administration [57]. Surprisingly, the anti-inflammatory Lyc did not affect the cyto-/adipokine secretion in this human adipocyte model. This is contrary to previous studies from our and other labs, which reported a reduced secretion of the pro-inflammatory cytokine IL- 6 and the macrophage attracting chemokine MCP-1 by Lyc treatment $(24 \mathrm{~h}, 2 \mu \mathrm{M})$ in murine and human adipocytes [58, 59]. In HFD-induced obese rats, Lyc supplementation showed increased adiponectin levels and decreased MCP-1 and IL-6 protein and mRNA levels in AT [56]. Nevertheless, our data show a marked reduction in LD accumulation and pro-inflammatory IL-6 by the combination Lyc/Res, suggesting that Res is the major driver of these anti-lipogenic and anti-inflammatory effects. This is in agreement with several studies, which indicate that treatment of mature SGBS adipocytes $(100 \mu \mathrm{M}, 48 \mathrm{~h})$ [19, 34], human AT explants $(50 \mu \mathrm{M}, 24 \mathrm{~h})$ [60] and of HFD-fed mice with Res (10 weeks) [37] can reduce the secretion of the pro-inflammatory cytokine IL-6 besides MCP-1 and IL-8.

The in-vitro observed decrease of adiponectin secretion with Res and Lyc/Res in human adipocytes is striking. Other groups reported that the secretion of this anti-inflammatory and insulin sensitizing adipocyte-specific hormone was increased when treating human SGBS adipocytes [34] and interleukin challenged primary adipocytes or AT explants [60] shortly (24 or $48 \mathrm{~h}$ ) with Res (up to $100 \mu \mathrm{M}$ ). We hypothesize that long-term exposure (14 days) with Res mainly inhibits the differentiation of adipocyte progenitor cells, and thus keeps adiponectin secretion 
levels close to the ones of undifferentiated progenitor cells. Further investigations are warranted to elucidate the mechanism underlying this observation. Altogether, these findings indicate that treatment with dietary bio-actives can alter the cyto-/adipokine release of adipocytes towards a less pro-inflammatory secretion profile.

In conclusion, this study corroborates that Res is the key driver of the improved functional features in differentiated human adipocytes independent of donor characteristics. Res modulated the balance between adipogenic, lipogenic and lipolytic gene expression, which resulted in a diminished LD accumulation, blunted basal and ISO-stimulated lipolysis and a reduced proinflammatory secretion profile. However, using combinations of bio-actives is indicated to be more effective in correcting AT dysfunction [21, 22, 61], by targeting partly distinct pathways. Moreover, supplementing Res together with other dietary bio-active nutrients (e.g. Lyc, polyphenols, n-3 PUFAs) could be advantageous for the treatment or prevention of obesityassociated metabolic diseases due to their effects on different metabolic/physiological pathways rather than their combined effects on adipose function.

\section{Acknowledgements}

We would like to thank Nathalie Richard for helping to perform Luminex experiments, Nicole Hoebers and Yvonne Essers for cell culture support and technical assistance at the university lab, Eliane Wandeler and Ann Sion for assistance with the ArrayScan ${ }^{\circledR}$ reader and protocol establishment. Özgür Sancak for critical review of the manuscript and Wim Saris for establishing and promoting the collaborations. 


\section{References}

1. Schweiger M, Schreiber R, Haemmerle G, Lass A, Fledelius C, Jacobsen P, Tornqvist H, Zechner R, Zimmermann R: Adipose triglyceride lipase and hormone-sensitive lipase are the major enzymes in adipose tissue triacylglycerol catabolism. The Journal of biological chemistry 2006, 281(52):4023640241.

2. Arner E, Westermark PO, Spalding KL, Britton T, Ryden M, Frisen J, Bernard S, Arner P: Adipocyte turnover: relevance to human adipose tissue morphology. Diabetes 2010, 59(1):105-109.

3. Arner P, Bernard S, Salehpour M, Possnert G, Liebl J, Steier P, Buchholz BA, Eriksson M, Arner E, Hauner $\mathrm{H}$ et al: Dynamics of human adipose lipid turnover in health and metabolic disease. Nature 2011, 478(7367):110-113.

4. Ng M, Fleming T, Robinson M, Thomson B, Graetz N, Margono C, Mullany EC, Biryukov S, Abbafati C, Abera SF et al: Global, regional, and national prevalence of overweight and obesity in children and adults during 1980-2013: a systematic analysis for the Global Burden of Disease Study 2013. Lancet 2014, 384(9945):766-781.

5. Hammarstedt A, Graham TE, Kahn BB: Adipose tissue dysregulation and reduced insulin sensitivity in non-obese individuals with enlarged abdominal adipose cells. Diabetology \& metabolic syndrome 2012, 4:42-42.

6. Guilherme A, Virbasius JV, Puri V, Czech MP: Adipocyte dysfunctions linking obesity to insulin resistance and type 2 diabetes. Nat Rev Mol Cell Biol 2008, 9(5):367-377.

7. Arner P, Langin D: Lipolysis in lipid turnover, cancer cachexia, and obesity-induced insulin resistance. Trends in endocrinology and metabolism: TEM 2014, 25(5):255-262.

8. Lowe CE, O'Rahilly S, Rochford JJ: Adipogenesis at a glance. Journal of cell science 2011, 124(16):2681-2686.

9. Galic S, Oakhill JS, Steinberg GR: Adipose tissue as an endocrine organ. Molecular and cellular endocrinology 2010, 316(2):129-139.

10. Weisberg SP, McCann D, Desai M, Rosenbaum M, Leibel RL, Ferrante AW, Jr.: Obesity is associated with macrophage accumulation in adipose tissue. The Journal of clinical investigation 2003, 112(12):1796-1808.

11. Kim CS, Park HS, Kawada T, Kim JH, Lim D, Hubbard NE, Kwon BS, Erickson KL, Yu R: Circulating levels of MCP-1 and IL-8 are elevated in human obese subjects and associated with obesity-related parameters. International journal of obesity 2006, 30(9):1347-1355.

12. Mraz M, Haluzik M: The role of adipose tissue immune cells in obesity and low-grade inflammation. The Journal of endocrinology 2014, 222(3):R113-127.

13. Hsu CL, Yen GC: Phenolic compounds: evidence for inhibitory effects against obesity and their underlying molecular signaling mechanisms. Molecular nutrition \& food research 2008, 52(1):53-61.

14. Kawada T, Kamei Y, Fujita A, Hida Y, Takahashi N, Sugimoto E, Fushiki T: Carotenoids and retinoids as suppressors on adipocyte differentiation via nuclear receptors. BioFactors 2000, 13(14):103-109.

15. Luisa Bonet M, Canas JA, Ribot J, Palou A: Carotenoids and their conversion products in the control of adipocyte function, adiposity and obesity. Archives of biochemistry and biophysics 2015, 572:112125.

16. Wang S, Moustaid-Moussa N, Chen L, Mo H, Shastri A, Su R, Bapat P, Kwun I, Shen CL: Novel insights of dietary polyphenols and obesity. The Journal of nutritional biochemistry 2014, 25(1):118.

17. Aguirre L, Fernández-Quintela A, Arias N, Portillo M: Resveratrol: Anti-Obesity Mechanisms of Action. Molecules 2014, 19(11):18632.

18. Warnke I, Jocken JW, Schoop R, Toepfer C, Goralczyk R, Schwager J: Combinations of bio-active dietary constituents affect human white adipocyte function in-vitro. Nutrition \& metabolism 2016, 13:84.

19. Zagotta I, Dimova EY, Debatin KM, Wabitsch M, Kietzmann T, Fischer-Posovszky P: Obesity and inflammation: reduced cytokine expression due to resveratrol in a human in vitro model of inflamed adipose tissue. Frontiers in pharmacology 2015, 6:79.

20. Bonet ML, Canas JA, Ribot J, Palou A: Carotenoids in Adipose Tissue Biology and Obesity. Subcellular biochemistry 2016, 79:377-414. 
21. Park HJ, Yang JY, Ambati S, Della-Fera MA, Hausman DB, Rayalam S, Baile CA: Combined effects of genistein, quercetin, and resveratrol in human and 3T3-L1 adipocytes. Journal of medicinal food 2008, 11(4):773-783.

22. Björk C, Wilhelm U, Mandrup S, Larsen BD, Bordoni A, Hedén P, Rydén M, Arner P, Laurencikiene $\mathrm{J}$ : Effects of selected bioactive food compounds on human white adipocyte function. Nutrition \& metabolism 2016, 13(1):1-10.

23. Arias N, Macarulla MT, Aguirre L, Milton I, Portillo MP: The combination of resveratrol and quercetin enhances the individual effects of these molecules on triacylglycerol metabolism in white adipose tissue. European journal of nutrition 2016, 55(1):341-348.

24. Ruiz-Ojeda FJ, Rupérez AI, Gomez-Llorente C, Gil A, Aguilera CM: Cell Models and Their Application for Studying Adipogenic Differentiation in Relation to Obesity: A Review. International journal of molecular sciences 2016, 17(7):1040.

25. Dubois SG, Floyd EZ, Zvonic S, Kilroy G, Wu X, Carling S, Halvorsen YDC, Ravussin E, Gimble JM, Prockop DJE et al: Isolation of Human Adipose-derived Stem Cells from Biopsies and Liposuction Specimens. In. Totowa, NJ; 2008: 69.

26. Jocken JW, Goossens GH, Popeijus H, Essers Y, Hoebers N, Blaak EE: Contribution of lipase deficiency to mitochondrial dysfunction and insulin resistance in hMADS adipocytes. International journal of obesity 2016, 40(3):507-513.

27. Verboven K, Hansen D, Moro C, Eijnde BO, Hoebers N, Knol J, Bouckaert W, Dams A, Blaak EE, Jocken JW: Attenuated atrial natriuretic peptide-mediated lipolysis in subcutaneous adipocytes of obese type 2 diabetic men. Clinical science 2016, 130(13):1105-1114.

28. Warnke I, Goralczyk R, Fuhrer E, Schwager J: Dietary constituents reduce lipid accumulation in murine C3H10 T1/2 adipocytes: A novel fluorescent method to quantify fat droplets. Nutrition \& metabolism 2011, 8(1):30.

29. Barber E, Sinclair AJ, Cameron-Smith D: Comparative actions of omega-3 fatty acids on in-vitro lipid droplet formation. Prostaglandins Leukot Essent Fatty Acids 2013, 89(5):359-366.

30. Rayalam S, Yang JY, Ambati S, Della-Fera MA, Baile CA: Resveratrol induces apoptosis and inhibits adipogenesis in 3T3-L1 adipocytes. Phytotherapy research : PTR 2008, 22(10):1367-1371.

31. Alberdi G, Rodríguez VM, Miranda J, Macarulla MT, Arias N, Andrés-Lacueva C, Portillo MP: Changes in white adipose tissue metabolism induced by resveratrol in rats. Nutrition \& metabolism 2011, 8:29-29.

32. Chang CC, Lin KY, Peng KY, Day YJ, Hung LM: Resveratrol exerts anti-obesity effects in high-fat diet obese mice and displays differential dosage effects on cytotoxicity, differentiation, and lipolysis in 3T3-L1 cells. Endocrine journal 2015.

33. Fischer-Posovszky P, Kukulus V, Tews D, Unterkircher T, Debatin KM, Fulda S, Wabitsch M: Resveratrol regulates human adipocyte number and function in a Sirt1-dependent manner. Am J Clin Nutr 2010, 92(1):5-15.

34. Rosenow A, Noben JP, Jocken J, Kallendrusch S, Fischer-Posovszky P, Mariman EC, Renes J: Resveratrol-induced changes of the human adipocyte secretion profile. Journal of proteome research 2012, 11(9):4733-4743.

35. Hu P, Zhao L, Chen J: Physiologically achievable doses of resveratrol enhance 3T3-L1 adipocyte differentiation. European journal of nutrition 2015, 54(4):569-579.

36. Chen S, Li Z, Li W, Shan Z, Zhu W: Resveratrol inhibits cell differentiation in 3T3-L1 adipocytes via activation of AMPK. Canadian journal of physiology and pharmacology 2011, 89(11):793-799.

37. Kim S, Jin Y, Choi Y, Park T: Resveratrol exerts anti-obesity effects via mechanisms involving downregulation of adipogenic and inflammatory processes in mice. Biochemical pharmacology 2011, 81(11):1343-1351.

38. Mercader J, Palou A, Bonet ML: Resveratrol enhances fatty acid oxidation capacity and reduces resistin and Retinol-Binding Protein 4 expression in white adipocytes. The Journal of nutritional biochemistry 2011, 22(9):828-834.

39. Yoshino J, Conte C, Fontana L, Mittendorfer B, Imai S, Schechtman KB, Gu C, Kunz I, Rossi Fanelli F, Patterson BW et al: Resveratrol supplementation does not improve metabolic function in nonobese women with normal glucose tolerance. Cell metabolism 2012, 16(5):658-664.

40. Konings E, Timmers S, Boekschoten MV, Goossens GH, Jocken JW, Afman LA, Muller M, Schrauwen P, Mariman EC, Blaak EE: The effects of 30 days resveratrol supplementation on adipose 
tissue morphology and gene expression patterns in obese men. International journal of obesity 2014, 38(3):470-473.

41. Poulsen MM, Vestergaard PF, Clasen BF, Radko Y, Christensen LP, Stodkilde-Jorgensen H, Moller N, Jessen N, Pedersen SB, Jorgensen JO: High-dose resveratrol supplementation in obese men: an investigator-initiated, randomized, placebo-controlled clinical trial of substrate metabolism, insulin sensitivity, and body composition. Diabetes 2013, 62(4):1186-1195.

42. Brasnyo P, Molnar GA, Mohas M, Marko L, Laczy B, Cseh J, Mikolas E, Szijarto IA, Merei A, Halmai R et al: Resveratrol improves insulin sensitivity, reduces oxidative stress and activates the Akt pathway in type 2 diabetic patients. The British journal of nutrition 2011, 106(3):383-389.

43. Bhatt JK, Thomas S, Nanjan MJ: Resveratrol supplementation improves glycemic control in type 2 diabetes mellitus. Nutrition research 2012, 32(7):537-541.

44. Timmers S, de Ligt M, Phielix E, van de Weijer T, Hansen J, Moonen-Kornips E, Schaart G, Kunz I, Hesselink MK, Schrauwen-Hinderling VB et al: Resveratrol as Add-on Therapy in Subjects With Well-Controlled Type 2 Diabetes: A Randomized Controlled Trial. Diabetes care 2016, 39(12):22112217.

45. Most J, Timmers S, Warnke I, Jocken JW, van Boekschoten M, de Groot P, Bendik I, Schrauwen P, Goossens GH, Blaak EE: Combined epigallocatechin-3-gallate and resveratrol supplementation for 12 wk increases mitochondrial capacity and fat oxidation, but not insulin sensitivity, in obese humans: a randomized controlled trial. Am J Clin Nutr 2016, 104(1):215-227.

46. Chang CC, Lin KY, Peng KY, Day YJ, Hung LM: Resveratrol exerts anti-obesity effects in high-fat diet obese mice and displays differential dosage effects on cytotoxicity, differentiation, and lipolysis in 3T3-L1 cells. Endocrine journal 2016, 63(2):169-178.

47. Gomez-Zorita S, Treguer K, Mercader J, Carpene C: Resveratrol directly affects in vitro lipolysis and glucose transport in human fat cells. Journal of physiology and biochemistry 2013, 69(3):585-593.

48. Shan T, Ren Y, Wang Y: Sirtuin 1 affects the transcriptional expression of adipose triglyceride lipase in porcine adipocytes. Journal of animal science 2013, 91(3):1247-1254.

49. Lasa A, Schweiger M, Kotzbeck P, Churruca I, Simon E, Zechner R, Portillo MP: Resveratrol regulates lipolysis via adipose triglyceride lipase. The Journal of nutritional biochemistry 2012, 23(4):379-384.

50. Szkudelska K, Nogowski L, Szkudelski T: Resveratrol, a naturally occurring diphenolic compound, affects lipogenesis, lipolysis and the antilipolytic action of insulin in isolated rat adipocytes. The Journal of steroid biochemistry and molecular biology 2009, 113(1-2):17-24.

51. Girousse A, Tavernier G, Valle C, Moro C, Mejhert N, Dinel A-L, Houssier M, Roussel B, BessePatin A, Combes $\mathrm{M}$ et al: Partial Inhibition of Adipose Tissue Lipolysis Improves Glucose Metabolism and Insulin Sensitivity Without Alteration of Fat Mass. PLoS biology 2013, 11(2):e1001485.

52. Timmers S, Konings E, Bilet L, Houtkooper RH, van de Weijer T, Goossens GH, Hoeks J, van der Krieken S, Ryu D, Kersten S et al: Calorie restriction-like effects of 30 days of resveratrol supplementation on energy metabolism and metabolic profile in obese humans. Cell metabolism 2011, 14(5):612-622.

53. Basu A, Imrhan V: Tomatoes versus lycopene in oxidative stress and carcinogenesis: conclusions from clinical trials. Eur J Clin Nutr 2007, 61(3):295-303.

54. Kaulmann A, Bohn T: Carotenoids, inflammation, and oxidative stress-implications of cellular signaling pathways and relation to chronic disease prevention. Nutrition research 2014, 34(11):907929.

55. Gouranton E, Aydemir G, Reynaud E, Marcotorchino J, Malezet C, Caris-Veyrat C, Blomhoff R, Landrier JF, Ruhl R: Apo-10'-lycopenoic acid impacts adipose tissue biology via the retinoic acid receptors. Biochimica et biophysica acta 2011, 1811(12):1105-1114.

56. Luvizotto RdAM, Nascimento AF, Imaizumi E, Pierine DT, Conde SJ, Correa CR, Yeum K-J, Ferreira ALA: Lycopene supplementation modulates plasma concentrations and epididymal adipose tissue mRNA of leptin, resistin and IL-6 in diet-induced obese rats. British Journal of Nutrition 2013, 110(10):1803-1809.

57. Luvizotto RA, Nascimento AF, Miranda NC, Wang XD, Ferreira AL: Lycopene-rich tomato oleoresin modulates plasma adiponectin concentration and mRNA levels of adiponectin, SIRT1, and FoxO1 in adipose tissue of obese rats. Human \& experimental toxicology 2015, 34(6):612-619. 
58. Gouranton E, Thabuis C, Riollet C, Malezet-Desmoulins C, El Yazidi C, Amiot MJ, Borel P, Landrier JF: Lycopene inhibits proinflammatory cytokine and chemokine expression in adipose tissue. The Journal of nutritional biochemistry 2011, 22(7):642-648.

59. Kanda H, Tateya S, Tamori Y, Kotani K, Hiasa K, Kitazawa R, Kitazawa S, Miyachi H, Maeda S, Egashira K et al: MCP-1 contributes to macrophage infiltration into adipose tissue, insulin resistance, and hepatic steatosis in obesity. The Journal of clinical investigation 2006, 116(6):1494-1505.

60. Olholm J, Paulsen SK, Cullberg KB, Richelsen B, Pedersen SB: Anti-inflammatory effect of resveratrol on adipokine expression and secretion in human adipose tissue explants. Int J Obes 2010, 34(10):1546-1553.

61. Siriwardhana N, Kalupahana NS, Cekanova M, LeMieux M, Greer B, Moustaid-Moussa N: Modulation of adipose tissue inflammation by bioactive food compounds. The Journal of nutritional biochemistry 2013, 24(4):613-623. 
Supplemental Material 


\section{Supplemental Results}

A

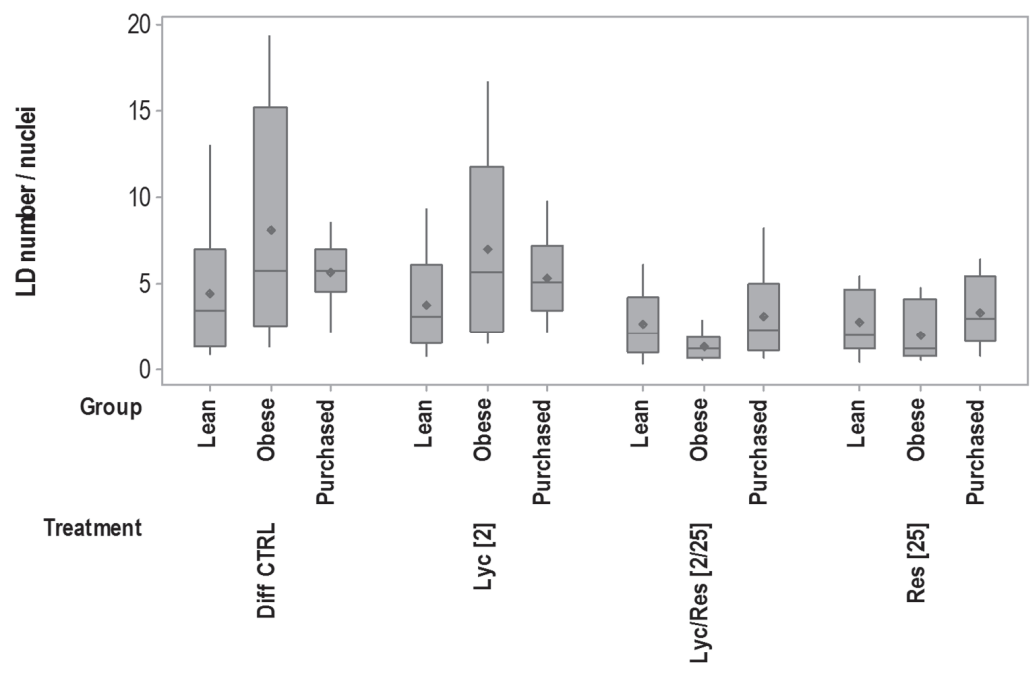

B

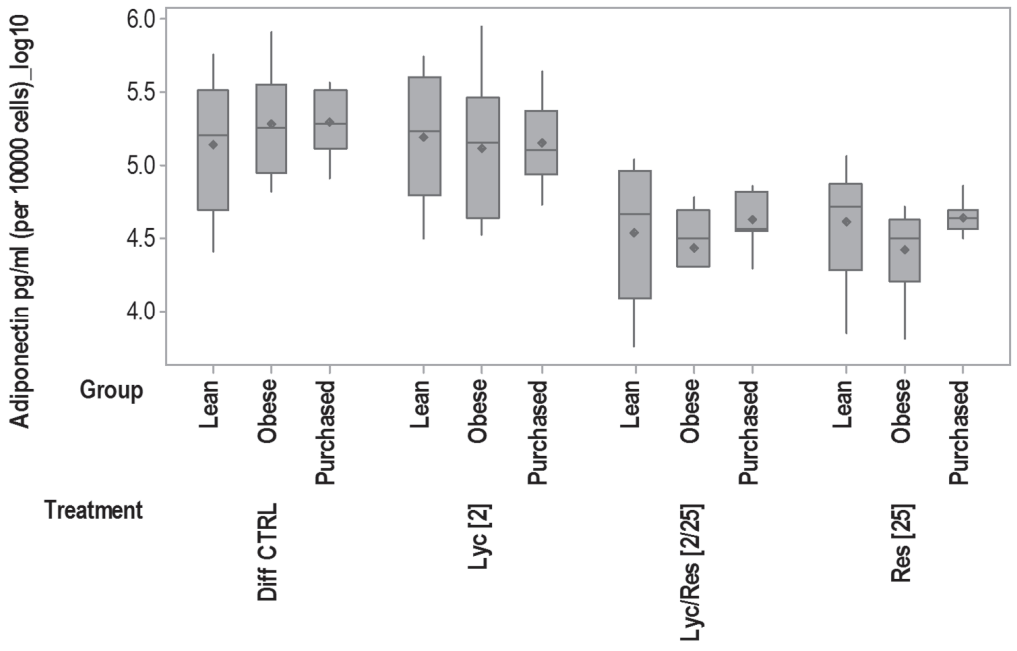


C

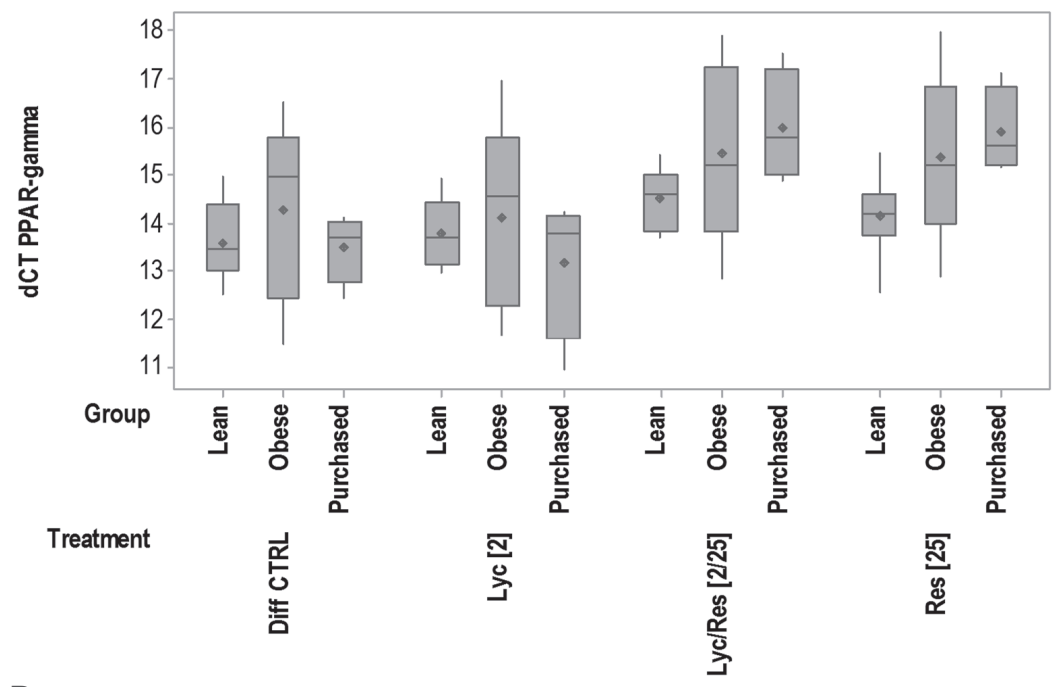

D

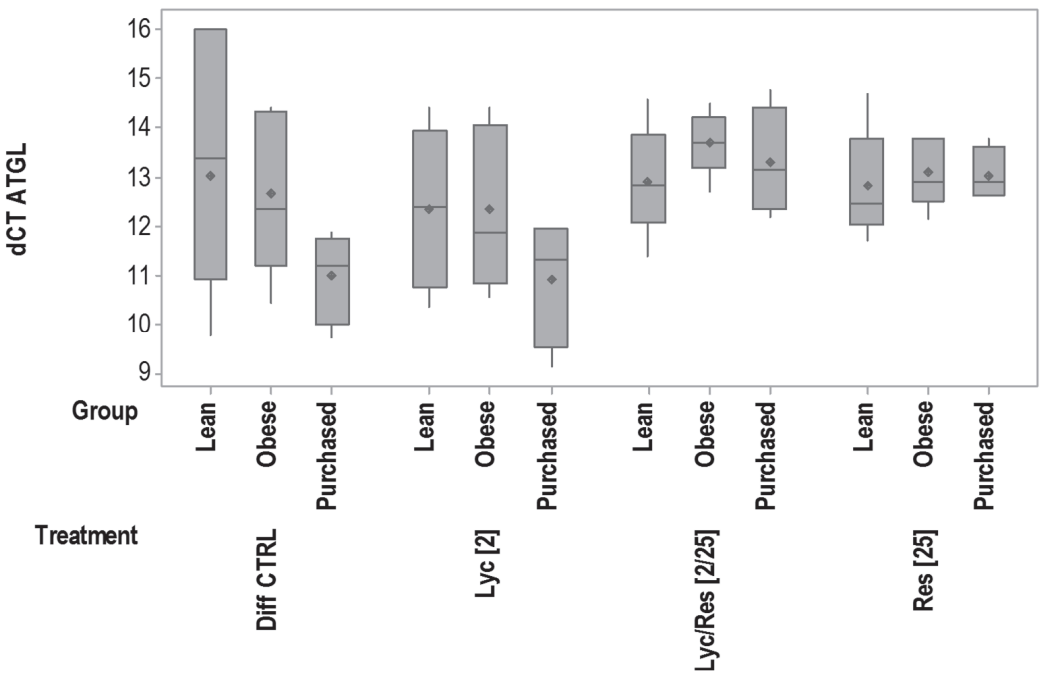

Supplemental Figure S4.1: Group independent treatment effects of Lyc and Res in in-vitro differentiated human adipocytes

hMADS cells from lean $(n=6)$ and obese $(n=4)$ donors and purchased HPAd $(n=4)$ were differentiated for 14 days in the presence of Lyc and Res alone or the combination thereof. Effects of Lyc $(2 \mu \mathrm{M})$, Res $(25 \mu \mathrm{M})$, and Lyc/Res $(2 / 25 \mu \mathrm{M})$ on LD number (normalized by nuclei number) (A), adiponectin secretion (log10 of $\mathrm{pg} / \mathrm{ml}$ per $10000 \mathrm{cells}$ ) and mRNA levels (as dCT) of PPARgamma (C) and ATGL (D) are depicted, compared to differentiation-only medium (Diff CTRL). Data are represented as boxplots (showing median, mean, $25 \%$ and $75 \%$ quartile, and SD, $n \geq 13$ donors). dCT: delta cycle threshold; CTRL: control; Diff: differentiation; hMADS: human multipotent adipose-derived stem cells; HPAd: human pre-adipocytes; LD: lipid droplet; SD: standard deviation; SEM: standard error of the mean. Statistical significance between groups was determined by ANOVA (significance level $\left({ }^{*}\right) p<0.05,\left(^{*} *\right) p<0.001$ ). 


\section{Lipid Droplet Parameters}

A

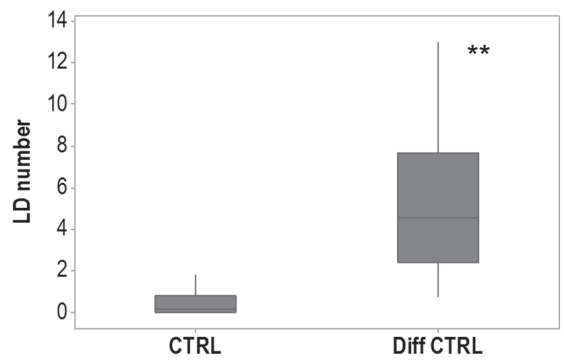

B

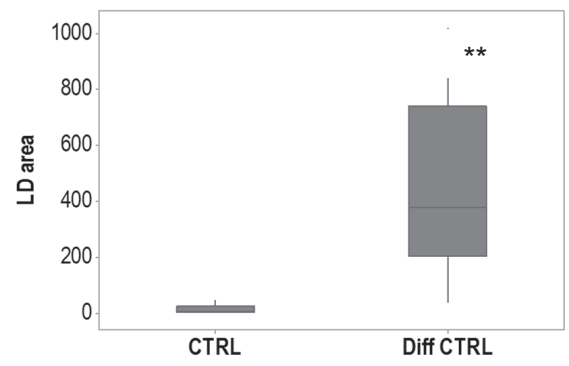

Controls [day 14]

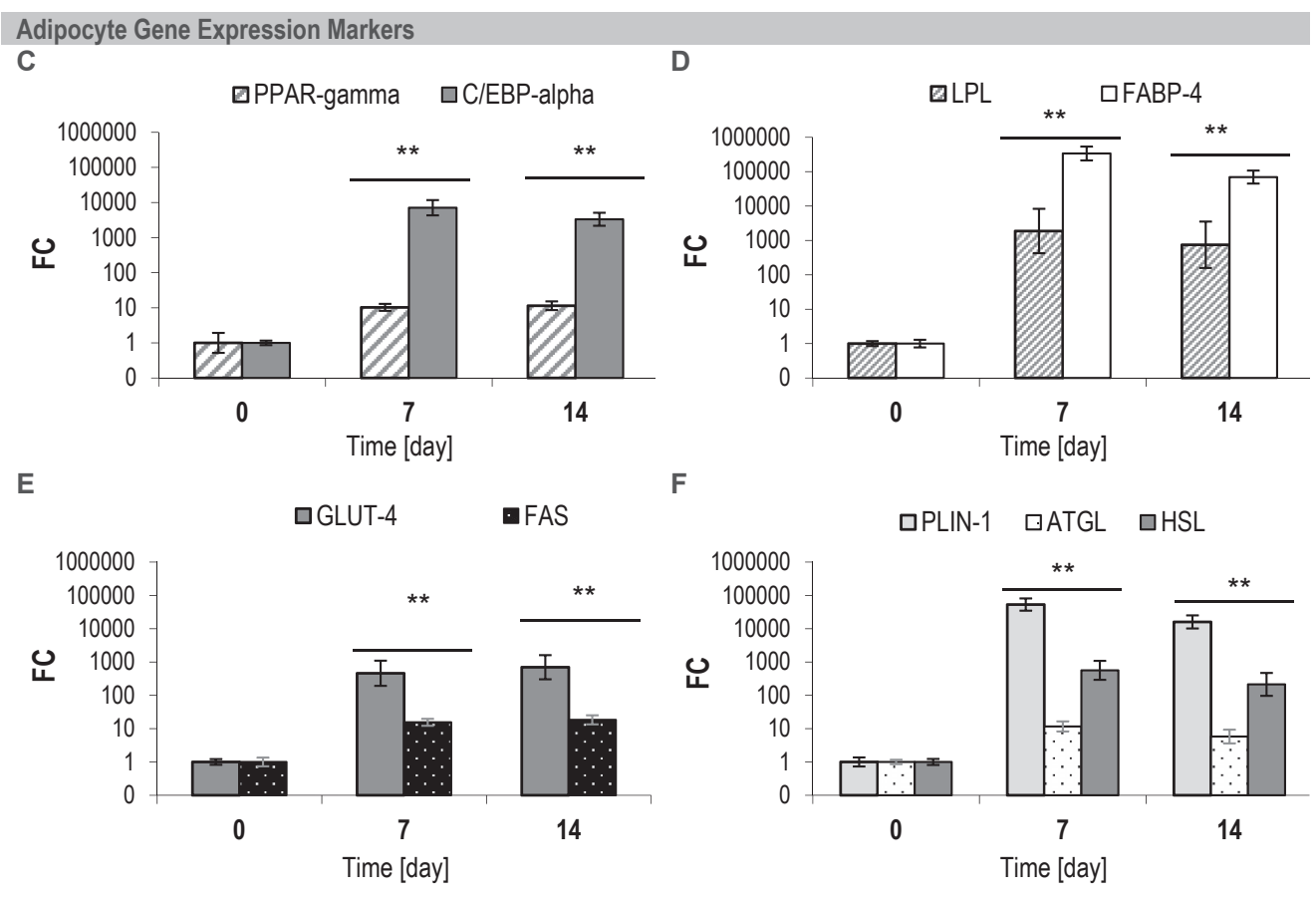

Supplemental Figure S4.2: Markers of in-vitro differentiated human adipocytes: Lipid droplet number and adipogenic, lipogenic and lipolytic gene expression profiles markers

hMADS cells from lean and obese donors and HPAd were differentiated for 14 days. Absolute overall means of the two LD parameters number (A) and area (B) for CTRL and Diff CTRL cells as detected with the ArrayScan ${ }^{\circledR}$ VTI HCS Reader (day 14, boxplots: median, mean, $25 \%$ and $75 \%$ quartile, and SD). mRNA levels of adipogenic transcription factors PPAR-gamma and C/EBP-alpha (C), lipogenesis markers GLUT-4, FAS (D), LPL and FABP-4 (E) and lipolysis associated proteins PLIN-1, HSL and ATGL (F) were determined by quantitative RT-PCR at day 0 , day 7 and 14. Data are expressed as overall FC \pm error (based on SEM) over baseline (day 0 , set as 1) ( $n \geq 13$ donors). CTRL: control; Diff: differentiation; FC: fold change; hMADS: human multipotent adipose-derived stem cells; HPAd: human pre-adipocytes, SEM: standard error of the mean. Genes: ATGL: Adipose triglyceride lipase; C/EBP-alpha: CCAAT/enhancer binding protein alpha; FABP-4: Fatty acid binding protein 4, FAS: Fatty acid synthase, GLUT-4: Glucose transporter 4; HSL: Hormone sensitive lipase; LPL: lipoprotein lipase; PLIN-1: Perilipin 1; PPARgamma: peroxisome proliferator-activated receptor gamma. Statistical significance was determined by paired Student's t-test comparing CTRL versus Diff CTRL (A-B) or day 0 versus day 7 or day $14(C-F)$ (significance level $\left.\left({ }^{*}\right) p<0.05,\left({ }^{*} *\right) p<0.001\right)$. 
Supplemental Table S4.1: Cellomics ${ }^{\mathrm{TM}}$ assay parameters for the analysis of LDs

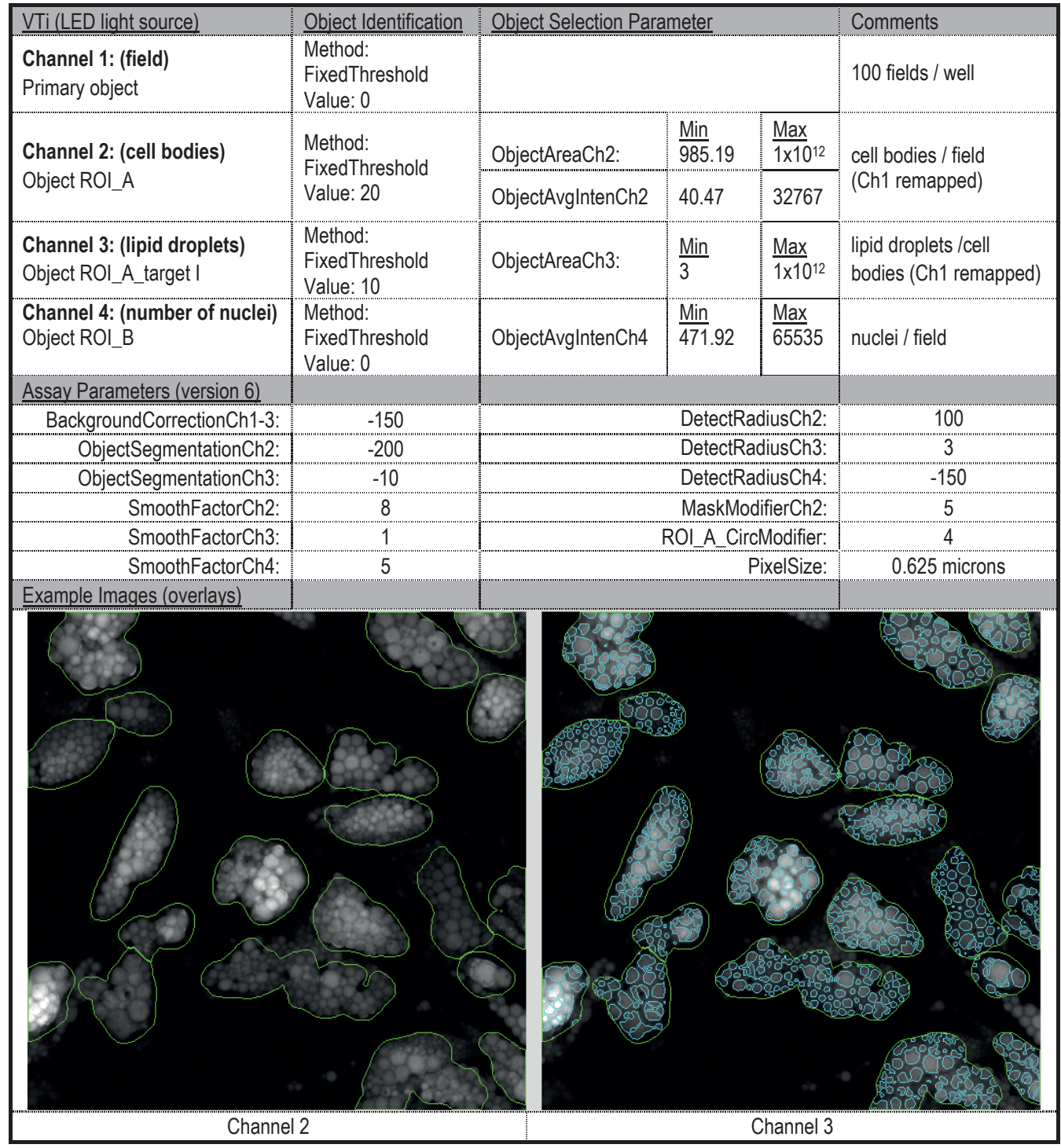

Displayed are values differing from the default settings in pixel number. Camera: ORCA-ER;1.00; Objective: 20x; ThermoFisher Bioapplication applied: Colocalization ${ }^{\circledR}$ V4; Normalized MEAN ROI_A Target I (Ch3) related features object count (= LD number), object total area (= LD area) and object total intensity (= LD intensity), describing the LD accumulation in treated cells, were calculated as percent of Diff CTRL per plate; Ch: channel; LD: lipid droplet; ROI: region of interest 


\section{CHAPTER 5}

Combined epigallocatechin-3-gallate and resveratrol supplementation for 12 wk increases mitochondrial capacity and fat oxidation, but not insulin sensitivity in obese humans: a randomized controlled trial 


\section{Abstract}

Background: The obese insulin resistant state is characterized by impairments in lipid metabolism. We previously showed that 3-day supplementation of combined epigallocatechin-3-gallate and resveratrol (EGCG+RES) increased energy expenditure, and improved the capacity to switch from fat towards carbohydrate oxidation with a high-fat mixed meal (HFMM) test in men. The present study aimed to investigate the longer-term effect of EGCG+RES supplementation on metabolic profile, mitochondrial capacity, fat oxidation, lipolysis, and tissue-specific insulin sensitivity.

Methods: In this randomized, double-blind study, 38 overweight and obese subjects (18 men, aged $38 \pm 2$ y, body-mass-index $29.7 \pm 0.5 \mathrm{~kg} / \mathrm{m}^{2}$ ) received either EGCG+RES (282 and $80 \mathrm{mg} / \mathrm{d}$, respectively) or placebo (PLA) for $12 \mathrm{wk}$. Before and after the intervention, oxidative capacity and gene expression were assessed in skeletal muscle. Fasting and postprandial (HFMM) lipid metabolism were assessed by using indirect calorimetry, blood sampling and microdialysis. Tissue-specific insulin sensitivity was assessed by a hyperinsulinemic-euglycemic clamp with $\left[6,6-{ }^{2} \mathrm{H}_{2}\right]$-glucose infusion.

Results: EGCG+RES supplementation did not affect the fasting plasma metabolic profile. Although wholebody fat mass was not affected, visceral adipose tissue mass tended to decrease after the intervention compared with PLA ( $\mathrm{P}_{\text {time* }}{ }^{*}$ treatment $\left.=0.09\right)$. EGCG+RES supplementation significantly increased oxidative capacity in permeabilized muscle fibers $\left(\mathrm{P}_{\text {time }}{ }^{*}\right.$ treatment $\left.<0.05, \mathrm{P}_{\mathrm{EGCG}+\mathrm{RES}}<0.05\right)$. Moreover, EGCG+RES reduced fasting $\left(\mathrm{P}_{\text {time*treatment }}=0.03\right)$ and postprandial respiratory quotient $\left(\mathrm{P}_{\text {time }}{ }^{*}\right.$ treatment $\left.=0.01\right)$ compared with placebo. Fasting and postprandial fat oxidation was not significantly affected by EGCG+RES ( $\mathrm{P}_{\mathrm{EGCG}+\mathrm{RES}}=0.46$ and $\mathrm{P}_{\mathrm{EGCG}+\mathrm{RES}}=0.38$, respectively) but declined after PLA ( $\mathrm{P}_{\mathrm{PLA}}=0.05$ and $\mathrm{P}_{\mathrm{PLA}}=0.03$, respectively). Energy expenditure was not altered $\left(\mathrm{P}_{\text {time*treatment }}=0.96\right)$. Furthermore, EGCG+RES supplementation attenuated the increase in plasma triacylglycerol concentration during the HFMM test that was observed after PLA $\left(\mathrm{P}_{\text {time*treatment }}=0.04, \mathrm{P}_{\mathrm{PLA}}=0.01\right)$. Finally, EGCG+RES had no effect on insulinstimulated glucose disposal, suppression of endogenous glucose production and lipolysis.

Conclusions: 12 weeks EGCG+RES supplementation increased mitochondrial capacity and stimulated fat oxidation compared with placebo, but this did not translate into increased tissue-specific insulin sensitivity in overweight and obese subjects. 


\section{Background}

The prevalence of obesity and related chronic diseases is continuously increasing [1]. Insulin resistance is a major risk factor for the progression of obesity towards chronic metabolic diseases, including cardiovascular disease and type 2 diabetes mellitus (T2DM) [2]. Reduced lipid storage capacity and impaired endocrine function of adipose tissue (AT) [3], and decreased mitochondrial capacity and accumulation of lipid-intermediates in skeletal muscle $[2,4,5]$ are closely associated with insulin resistance.

Current treatment strategies to control the progression of chronic diseases are mainly focused on lifestyle, pharmacological or surgical interventions. However, these interventions showed large inter-individual variability in response [6], which underscores the need for additional strategies to optimize the prevention of obesity-associated metabolic disorders.

Polyphenols were identified as dietary ingredients with antioxidant properties decades ago. More recently, they were also implicated in the prevention of T2DM and cardiovascular diseases. As such, epigallocatechin-3-gallate (EGCG), which is most abundant in green tea, and resveratrol (RES), which is present in grape skin, red wine and peanuts, have been implicated in the prevention of body weight gain and insulin resistance in rodents fed obesogenic diets [7-9]. In animal models of obesity, EGCG has been shown to reduce the absorption of intestinal lipids and to increase lipid catabolism, possibly by inhibiting catechol-O-methyltransferase [10] or by activating AMP kinase (AMPK) [11]. RES has been suggested to stimulate mitochondrial biogenesis by activating sirtuin 1 (SIRT1) and peroxisome proliferator-activated receptor $\gamma$ coactivator $1-\alpha(\mathrm{PGC} 1 \alpha)[7,8]$.

Human studies have shown that supplementation with both EGCG (or EGCG-rich products) and RES improved markers of insulin sensitivity (HOMA-IR, EGCG [12], RES [13]), reduced plasma markers of oxidative stress (total antioxidative status) and systemic inflammation (TNF- $\alpha$, EGCG [12], RES [13]), stimulated postprandial fat oxidation (EGCG [14]) and reduced body weight (EGCG [15], RES [16]). However, conflicting data in humans have also been reported [17-19].

We recently postulated that a combination of polyphenols with distinct mechanisms of action may have additional and/or synergistic effects, which may result in physiologically relevant effects on fat oxidation. Indeed, we showed that supplementation of a specific combination of EGCG and RES (EGCG+RES) for 3 days significantly increased resting and postprandial energy expenditure (EE), and resulted in a more pronounced increase in respiratory quotient (RQ) after a high-fat mixed meal (HFMM) compared with placebo (PLA) in men [20]. Importantly, these effects were not observed after single EGCG supplementation [21]. 
Here, we investigated the effects of longer-term combined EGCG+RES supplementation on metabolic profile, skeletal muscle oxidative capacity, fat oxidation, lipolysis, and peripheral, hepatic, and AT insulin sensitivity in overweight and obese, non-diabetic subjects.

\section{Methods}

\section{Subjects}

42 untrained $(<3 \mathrm{~h}$ organized sports activities/wk), weight-stable $(<2 \mathrm{~kg}$ body weight change 3 months before inclusion) overweight and obese (BMI $\left.>25 \mathrm{~kg} / \mathrm{m}^{2}\right)$, Caucasian men $(\mathrm{n}=21)$ and women $(n=21)$ aged between 20 and 50 y with normal glucose tolerance (fasting glucose $<6.1$ mmol/L, 2 h-glucose $<7.8 \mathrm{mmol} / \mathrm{L}$ ), normal blood pressure (diastolic: 60-90 mmHg; systolic: 100$140 \mathrm{mmHg}$ ) were included in this study. Subjects were not allowed to donate blood or use any medication or supplements that might interfere with study outcomes for 3 months before entering the study. Exclusion criteria were pregnancy, menopause, lactation and any reported (history of) chronic inflammatory, cardiovascular, hepatic, pulmonary, renal, or gastrointestinal disease. Intakes of caffeine $(<600 \mathrm{mg} / \mathrm{d})$, green tea $(<3 \mathrm{cups} / \mathrm{d})$, alcohol $(<20 \mathrm{~g} / \mathrm{d})$, grapes and peanuts (occasional consumption) had to be limited.

\section{Study design and randomization}

In this randomized, double-blind, placebo-controlled, parallel intervention trial, subjects received either a combination of EGCG and RES supplements (EGCG+RES; $282 \mathrm{mg} / \mathrm{d}$ and $80 \mathrm{mg} / \mathrm{d}$, respectively) or PLA (partly hydrolyzed microcrystalline cellulose-filled capsules) for a period of 12 wk to assess effects of EGCG+RES supplementation on tissue-specific insulin sensitivity (primary outcome) and metabolic profile, skeletal muscle oxidative capacity, fat oxidation and lipolysis (secondary outcomes). An independent researcher executed a block-wise randomization procedure and packed the supplements according to a computer-generated randomization plan (Microsoft Excel 2011 for Macintosh). The supplementation period started the day after the last baseline measurement in week 0 and was continued throughout measurements in week 12 . Subjects were instructed to maintain their habitual lifestyle pattern throughout the study. In total, subjects were asked to visit the university 10 times for medical screening, 3 clinical investigation days (CIDs) before the start of supplementation (within 7 days), 3 control visits during the supplementation period and 3 CIDs in the last week of supplementation (within 7 days). CIDs comprised skeletal muscle biopsies combined with dual energy X-ray absorptiometry (CID1), HFMM test (CID2), and hyperinsulinemic-euglycemic clamp (CID3) and were separated by $\geq 1$ day. Control visits for compliance, side effects and to provide new capsules were scheduled in 
week 2, 4, 8 of intervention. During week 0 and 12, subjects were asked to complete 3 -day food records ( 2 weekdays, 1 weekend day) and during week 4 a 1-day food record (1 weekday) to monitor and control for changes in dietary habits. An experienced dietitian checked the food records and discussed these with the subjects in case of incomplete or missing information. Energy and nutrient intake were analyzed using the Dutch Food Composition Dataset (NEVO: National Institute for Public Health and the Environment, Ministry of Health, Welfare and Sport, Netherlands).

\section{Clinical investigation days (CIDs)}

Two days before the CIDs, subjects were asked to refrain from intense physical activity and alcohol consumption. Meal intake during the evening before each CIDs was standardized per subject by providing the same meal. The first meal was provided ad libitum ( $740 \pm 34 \mathrm{kcal}, 48 \pm 2 \%$ of energy from fat) and subjects were asked to keep the portion size constant at all subsequent CIDs. After an overnight fast, subjects came to the university by car or public transport. Both before and after the intervention, a two-step hyperinsulinemic-euglycemic clamp and a HFMM test were performed, and a skeletal muscle biopsy was collected. All procedures were executed in a resting, half-supine position.

\section{Dual energy X-ray absorptiometry and skeletal muscle biopsy (CID1)}

First, body composition was measured by dual energy X-ray absorptiometry with the use of the 3compartment model (Hologic Corp.). Next, skeletal muscle (M. vastus lateralis) biopsy samples were taken under local anesthesia during fasting conditions before and after the 12-wk intervention period. One portion ( $\sim 30 \mathrm{mg}$ ) was used for high-resolution respirometry [22], which allows for the determination of oxidative capacity ex vivo by quantifying the oxygen consumption with the use of the Oxygraph-2k (OROBOROS Instruments). The other portion was directly frozen in isopentane and stored at $-80^{\circ} \mathrm{C}$ until further analyses. Protein quantification of mitochondrial oxidative phosphorylation (OxPhos) complexes was performed by SDS-PAGE followed by Western blot analysis with the use of nitrocellulose membranes (Trans-blot ${ }^{\circledR}$ Turbo ${ }^{\mathrm{TM}}$ transfer system, Bio-Rad). Blots were probed with Total OxPhos Antibody Cocktail (Mitoscience/Abcam) and a secondary Infrared dye680/700 conjugated donkey anti-mouse antibody (Licor/Invitrogen). Antigen-antibody complexes were visualized by using an Odyssey Infrared Imaging System (LICOR Biosciences).

A detailed description for skeletal muscle microarray analysis and lipid composition is provided in the Supplemental Methods. Briefly, for microarray analysis, $100 \mathrm{ng}$ intact total RNA was extracted from skeletal muscle biopsy samples by using the Trizol method (Qiagen) and processed 
by applying the GeneChip ${ }^{\circledR}$ WT PLUS Reagent Kit and Human Transcriptome Array (HTA) 2.0 GeneChips $^{\circledR}$ (Affymetrix) according to the manufacture's instructions. Functional data analysis was based on a false-discovery rate q-value $<0.2$ on the filtered data set (interquartile range (IQR) $>0.2(\log 2)$, intensity $>20,>5$ arrays, $>5$ probes/gene).

For the determination of lipid composition, total lipids were extracted after lyophilisation of skeletal muscle tissue. Diacylglycerol (DAG) and triacylglycerol (TAG) were separated by thinlayer chromatography and fatty acid profiles were determined on an analytical gas chromatograph, as described previously [5].

\section{Microdialysis (CID2)}

Four microdialysis probes (CMA 60; CMA Microdialysis) were inserted under local anesthesia, 2 in the medial portion of the gastrocnemius muscle of both legs and two in the subcutaneous adipose tissue $6-8 \mathrm{~cm}$ left and right from the umbilicus in male subjects $(\mathrm{n}=17$; age: $40.1 \pm 2.0$ years; BMI: $30.0 \pm 0.8 \mathrm{~kg} / \mathrm{m}^{2}$; HOMA-IR: 2.1 \pm 0.4 ) as described previously [23]. After insertion, $90 \mathrm{~min}$ were allowed for tissue recovery from the insertion trauma. Throughout the HFMM test (CID2), in each tissue, one probe was perfused at $0.3 \mu \mathrm{L} / \mathrm{min}$ with Ringer solution to collect microdialysate for analyses of glycerol, glucose, pyruvate, and lactate concentrations by means of CMA enzymatic assay kits on a CMA 600 microdialysis analyzer.

The contralateral probe was perfused at $5.0 \mu \mathrm{L} / \mathrm{min}$ with Ringer solution, supplemented with 50 $\mathrm{mmol} / \mathrm{L}$ ethanol, to assess the ethanol outflow/inflow ratio as an indicator of local blood flow. Ethanol concentrations were measured spectrophotometrically at $340 \mathrm{~nm}$ by using a standard ethanol assay kit (Boehringer).

\section{HFMM test (CID2)}

After inserting a cannula into the antecubital vein, substrate oxidation was measured for $30 \mathrm{~min}$ under fasting conditions $(\mathrm{t}=0)$ and for $4 \mathrm{~h}$ after the ingestion of a liquid HFMM (625 kcal, 61\% of energy from fat, $33 \%$ of energy from carbohydrate, $6 \%$ of energy from protein), which was consumed within $5 \mathrm{~min}$ at $\mathrm{t}=0$. Blood samples were taken under fasting $(\mathrm{t}=0 \mathrm{~min})$ and postprandial $(\mathrm{t}=30,60,90,120,150,180,210$ and $240 \mathrm{~min})$ conditions. EE and substrate oxidation were measured by indirect calorimetry by using the open-circuit ventilated hood system (Omnical, Maastricht University) and were calculated according to the formulas of Weir [24] and Frayn [25], respectively.

\section{Hyperinsulinemic-euglycemic clamp (CID3)}

A 2-step hyperinsulinemic-euglycemic clamp with $\left[6,6-{ }^{2} \mathrm{H}_{2}\right]$-glucose infusion (tracer, Cambridge Isotope Laboratories) was performed to assess the rate of disappearance $\left(\mathrm{R}_{\mathrm{d}}\right)$, non-oxidative 
glucose disposal (NOGD) and endogenous glucose production (EGP) [22]. First, a cannula was inserted into the antecubital vein. A second cannula was inserted into a superficial dorsal hand vein for the sampling of arterialized blood (by using a hot-box with air circulating at $\sim 50^{\circ} \mathrm{C}$ ). After the administration of a bolus-injection of $2.4 \mathrm{mg}\left[6,6-{ }^{2} \mathrm{H}_{2}\right]$-glucose $/ \mathrm{kg}$, a continuous $\left[6,6-{ }^{2} \mathrm{H}_{2}\right]$ glucose infusion was started at $0.04 \mathrm{mg} /\left(\mathrm{kg}^{*} \mathrm{~min}\right)$ and continued throughout the measurement. After $2 \mathrm{~h}$, insulin infusion was started at $10 \mathrm{mU} /\left(\mathrm{m}^{2 *} \min \right)$ for $2 \mathrm{~h}$, followed by $40 \mathrm{mU} /\left(\mathrm{m}^{2 *} \mathrm{~min}\right)$ insulin for the last $2 \mathrm{~h}$ to suppress lipolysis and EGP. By a variable co-infusion of a $20 \%$-glucose solution, enriched to $1.92 \mathrm{mg} / \mathrm{mL}$ tracer, blood glucose concentrations were maintained at 5.0 $\mathrm{mmol} / \mathrm{L}$. During the last $30 \mathrm{~min}$ of each insulin-infusion step $\left(0,10\right.$ and $\left.40 \mathrm{mU} /\left(\mathrm{m}^{2 *} \mathrm{~min}\right)\right)$ blood samples were collected and substrate oxidation was measured by using indirect calorimetry (described in the section entitled 'HFFM test (CID2)') to assess glucose kinetics. Kinetics of $\mathrm{R}_{\mathrm{d}}$ and NOGD were calculated during 0 and $40 \mathrm{mU} /\left(\mathrm{m}^{2 *} \mathrm{~min}\right)$ insulin-infusion, respectively as absolute increase between these steps $\left(\Delta \mu \mathrm{mol} /\left(\mathrm{kg}^{*} \mathrm{~min}\right)\right)$, whereas calculations for insulinmediated suppression of EGP and free fatty acids (FFA) were performed during 0 and 10 $\mathrm{mU} /\left(\mathrm{m}^{2 *} \mathrm{~min}\right)$ insulin-infusion, respectively, as relative suppression during 10 compared to 0 $\mathrm{mU} /\left(\mathrm{m}^{2 *} \min \right)(\%)$.

\section{Biochemistry}

Blood was collected into pre-chilled tubes, centrifuged $\left(1000 \mathrm{xg} ; 10 \mathrm{~min} ; 4^{\circ} \mathrm{C}\right)$ and plasma was snap-frozen in liquid nitrogen and stored at $-80^{\circ} \mathrm{C}$ until analyses.

To check for treatment compliance, plasma EGCG and RES concentrations were determined by liquid chromatography-mass spectrometry (LC-MS, for details see Supplemental Methods). In brief, for the measurement of free EGCG, $500 \mu \mathrm{L}$ of plasma was combined with the same volume of an ascorbic acid/EDTA stabilizing-buffer. For analyses, an internal standard was added, followed by a $2 x$ liquid-liquid extraction. Total RES and total dihydro-RES were measured as sum of aglycone and conjugated forms ( $\beta$-glucuronidase digestion required) after the addition of labelled internal standard by a liquid-liquid extraction. After centrifugation, an aliquot of the organic phase was evaporated to dryness, re-dissolved in injection solvent, and analyzed by using LC-MS systems. The isotopic enrichment of plasma glucose was determined by electron ionization gas chromatography-mass spectrometry and expressed as tracer-to-tracee ratio for steady-state calculations of $\mathrm{R}_{\mathrm{d}}$, NOGD and EGP, as described previously [22].

Plasma glucose, lactate, FFA, TAG, total cholesterol, and HDL-cholesterol (HDL-c) concentrations were determined with an automated spectrophotometer (ABX Pentra 400 autoanalyzer, Horiba ABX) by using enzymatic colorimetric kits. LDL-cholesterol (LDL-c) concentration was calculated by the Friedewald-equation [26]. Plasma glycerol concentrations 
were measured with an enzymatic assay (Enzytec Glycerol, Roche Biopharm) automated on a Cobas Fara spectrophotometric autoanalyzer (Roche Diagnostics). Circulating plasma concentrations of insulin, adiponectin and leptin were analyzed by using commercially available radioimmunoassay kits (Human Insulin specific radioimmunoassay, Human Adiponectin radioimmunoassay, Human Leptin radioimmunoassay, Millipore Corp.). Plasma concentrations of inflammatory markers (IL-6, IL- 8 and TNF- $\alpha$ ) were determined by using a multiplex ELISA (Human ProInflammatory II 4-Plex Ultra-Sensitive Kit, Meso Scale Diagnositics).

\section{Supplements}

The supplements were commercially available and were provided by Pure Encapsulations Inc. All the capsules were manufactured, tested, and checked in accordance to standards of the European GMP requirements.

EGCG capsules contained 94\% epigallocatechin-3-gallate (141 mg/capsule, lot 3570112) and RES capsules contained 20\% trans-resveratrol (40 mg trans-resveratrol in Polygonum cuspidatum extract/capsule, lot 1611011). The doses of the supplements were based on previous study outcomes, in which these doses altered markers of substrate and energy metabolism (Timmers et al. [13]: $150 \mathrm{mg}$ RES/d; Most et al. [20]: $200 \mathrm{mg}$ RES/d, $282 \mathrm{mg}$ EGCG/d). Higher concentrations appear to have no (additional) benefit (Poulsen et al. [17]: $1500 \mathrm{mg} \mathrm{RES/d}$; Brown et al. [19]: 800 mg EGCG/d). Two lots of placebo capsules (microcrystalline cellulose, lot 7150712 and 7160712 ) were used for blinding. PLA capsules were encapsulated identically to the active supplements and filled with microcrystalline cellulose and tan/brown and pink/red powder, respectively, to blind both supplements. Capsules were packed into white opaque boxes, labeled per subject without indication of the content. One capsule of each supplement (EGCG and RES or both blinded PLA capsules) was ingested during breakfast and dinner. This regimen was maintained until the last measurement. On CIDs, subjects ingested the capsules before arrival at the university (between 7 and $8 \mathrm{am}$ ). After completion of the study, returned capsules were counted for compliance and the stability of polyphenols was assessed by measuring EGCG and RES via HPLC in capsules stored for 3 months, according to the manufacture's recommendations. The supplements were considered safe and well-tolerated [20].

\section{Study approval}

All of the subjects gave written informed consent for participation in this study, which was reviewed and approved by the local medical ethical committee of Maastricht University Medical Center $^{+}$. This trial was registered at clinicaltrials.gov (NCT02381145). All procedures were conducted according to the Declaration of Helsinki. 


\section{Statistics}

The sample size $(n=42)$ was calculated to detect a physiological relevant change in insulin sensitivity of $20 \%$, with an SD of $4 \%$, a power of $80 \%$, assuming a significance level of $\alpha=0.05$ $(n=34)$, and a drop-out of $20 \%(n=8)$. All of the data are expressed as means \pm SEMs. Data for the HFMM test are expressed as AUCs to define the total measurement period during this test (fasting and postprandial), and as incremental AUCs to define specifically the postprandial response (diet-induced effect compared with fasting), both calculated by the trapezoid method. Variables were tested for normality by using Shapiro-Wilk-test and showed a normal distribution. Differences in subjects' characteristics at baseline were tested by Student's unpaired t-test. Data were analyzed by using a 2-factor repeated-measures ANOVA, with time [before intervention (pre) and after intervention (post)] and treatment (PLA, EGCG+RES) as factors. $\mathrm{P}<0.05$ was considered to be significant and $\mathrm{P}<0.10$ was considered to show a trend. In case of a significant time*treatment interaction $\left(\mathrm{P}_{\text {time*treatment }}\right)$, post-hoc analyses with Bonferroni correction were applied to identify significant within-group effects (PEGCG+RES, PPLA). In case of clinically relevant baseline differences between treatment groups (for fasting insulin, IL-6, mitochondrial capacity of state 3 respiration, and protein expression of OxPhos complexes III and V; for all $>20 \%$ baseline difference), the baseline value was included as covariate in univariate ANOVA, with the change over time as dependent variable and treatment as between-subject factor. In the ANOVA model, no interactions between sex and the primary and secondary outcomes were observed. Statistical analysis was performed by using SPSS 19.0 (IBM Corp.) for Macintosh.

\section{Results}

\section{Subject characteristics and plasma biochemistry}

42 overweight and obese men and women volunteered to participate in this study (August 2012 December 2013). In total, 4 subjects (3 men, 1 women) did not complete the study due to traveling abroad $(n=1)$, re-employment $(n=1)$ or non-compliance with respect to supplementation or changing lifestyle ( $\mathrm{n}=2)$. Characteristics of the 38 subjects (18 men, 20 women) who completed the study are summarized in Table 5.1. There were no significant differences between the EGCG+RES ( $n=18,8$ men) and PLA ( $n=20,10$ men) group with respect to baseline characteristics or self-reported moderate or intense physical activity (EGCG+RES: $1.4 \pm 0.3 \mathrm{~h} / \mathrm{wk}$; PLA: $1.6 \pm 0.2$ $\mathrm{h} / \mathrm{wk} ; \mathrm{P}=0.78)$. 
Table 5.1: Subjects' characteristics at baseline (week 0)

\begin{tabular}{lccc}
\hline & $\begin{array}{c}\text { PLA } \\
(\mathrm{n}=20)\end{array}$ & $\begin{array}{c}\text { EGCG+RES } \\
(\mathrm{n}=18)\end{array}$ & $\begin{array}{c}P \\
(\mathrm{t} \text {-test })\end{array}$ \\
\hline Age, years & $38.7 \pm 2.2$ & $36.1 \pm 2.2$ & 0.43 \\
BMI, kg/m² & $29.5 \pm 0.7$ & $29.9 \pm 0.6$ & 0.67 \\
Waist-hip-ratio & $0.87 \pm 0.02$ & $0.87 \pm 0.02$ & 0.78 \\
Systolic blood pressure, $\mathrm{mmHg}$ & $114 \pm 2$ & $117 \pm 2$ & 0.27 \\
Diastolic blood pressure, $\mathrm{mmHg}$ & $76 \pm 2$ & $76 \pm 2$ & 0.86 \\
Fasting plasma glucose, $\mathrm{mmol} / \mathrm{L}$ & $5.10 \pm 0.08$ & $5.19 \pm 0.09$ & 0.44 \\
2-h Glucose, mmol/L & $5.38 \pm 0.21$ & $5.34 \pm 0.24$ & 0.92 \\
HbA1c, \% & $5.15 \pm 0.06$ & $5.12 \pm 0.06$ & 0.76 \\
Hemoglobin, mmol/L & $8.9 \pm 0.1$ & $8.6 \pm 0.2$ & 0.22 \\
Hematocrit, L/L & $0.43 \pm 0.01$ & $0.39 \pm 0.02$ & 0.18 \\
Creatinine, $\mu \mathrm{mol} / \mathrm{L}$ & $79.5 \pm 3.7$ & $74.2 \pm 2.7$ & 0.29 \\
ALT, U/L & $27.9 \pm 4.4$ & $26.9 \pm 2.2$ & 0.86 \\
\hline
\end{tabular}

Values are means \pm SEMs. Data were analyzed by using a Student's unpaired t-test. $P<0.05$ was considered significant for the difference between PLA and EGCG+RES groups. ALT, alanine-aminotransferase, BMI, body mass index; EGCG+RES, epigallocatechin-3-gallate plus resveratrol; $\mathrm{HbA1c}$, glycated hemoglobin; PLA, placebo, 2-h Glucose, plasma glucose concentration two hours after oral glucose ingestion.

Compliance was confirmed by counting returned supplements $(<3 \%$ of supplements were returned). Supplements were well tolerated and no adverse events were reported. Importantly, plasma concentrations of EGCG, RES and dihydro-RES were significantly increased in all subjects who had received EGCG+RES (Table 5.2), which indicated compliance to the supplementation regimen. No significant changes in fasting plasma concentrations of glucose, insulin, lactate, glycerol, FFAs, or TAG were observed (Table 5.2, Figures 5.1A-F). Likewise, fasting plasma concentrations of leptin, adiponectin, and the inflammatory markers IL-6, IL-8 and TNF- $\alpha$ were not significantly affected by the intervention (Table 5.2). Furthermore, fasting plasma concentrations of total cholesterol, HDL-c and LDL-c were not significantly changed after $12 \mathrm{wk}$ of EGCG+RES-supplementation compared with PLA. However, the ratio of total cholesterol/HDL-c, a marker for myocardial infarction risk [27], tended to decrease after EGCG+RES supplementation but not after PLA ( $\mathrm{P}_{\text {time*treatment }}=0.03, \mathrm{P}_{\mathrm{EGCG}+\mathrm{RES}}=0.09, \mathrm{P}_{\mathrm{PLA}}=0.13$, Table 5.2). Adjustment for clinically relevant differences in baseline values (fasting insulin and IL-6) did not alter the outcome of the analysis ( $>20 \%$ baseline difference). 
Table 5.2: Fasting plasma biochemistry before and after 12 wk of supplementation with EGCG+RES or PLA

\begin{tabular}{|c|c|c|c|c|c|c|c|}
\hline & \multicolumn{2}{|c|}{$\begin{array}{c}\text { PLA } \\
(n=20)\end{array}$} & \multicolumn{2}{|c|}{$\begin{array}{c}\text { EGCG+RES } \\
(n=18)\end{array}$} & \multirow{2}{*}{$\begin{array}{c}\text { Time } \\
\mathrm{P}\end{array}$} & \multirow{2}{*}{$\begin{array}{c}\text { Treatment } \\
\mathrm{P}\end{array}$} & \multirow{2}{*}{$\begin{array}{c}\text { Time*treatment } \\
\text { P }\end{array}$} \\
\hline & Week 0 & Week 12 & Week 0 & Week 12 & & & \\
\hline EGCG, nmol/mL & $<5.45$ & $<5.45$ & $<5.45$ & $32.7 \pm 13.1^{*}$ & & & \\
\hline $\mathrm{RES}, \mathrm{nmol} / \mathrm{mL}$ & $<43.8$ & $<43.8$ & $<43.8$ & $1201 \pm 220$ * & & & \\
\hline $\begin{array}{l}\text { Dihydro-RES, } \\
\mathrm{nmol} / \mathrm{mL}\end{array}$ & $<43.4$ & $<43.4$ & $<43.4$ & $834 \pm 122$ * & & & \\
\hline Glucose, $\mathrm{mmol} / \mathrm{L}$ & $5.0 \pm 0.1$ & $5.1 \pm 0.1$ & $5.1 \pm 0.1$ & $5.1 \pm 0.1$ & 0.75 & 0.61 & 0.69 \\
\hline Insulin, mU/L & $10.1 \pm 1.5$ & $10.0 \pm 1.0$ & $8.1 \pm 1.0$ & $7.4 \pm 0.6$ & 0.54 & 0.10 & 0.67 \\
\hline HOMA-IR & $2.3 \pm 0.4$ & $2.2 \pm 0.2$ & $1.8 \pm 0.2$ & $1.7 \pm 0.1$ & 0.57 & 0.12 & 0.74 \\
\hline FFAs, $\mu \mathrm{mol} / \mathrm{L}$ & $553 \pm 33$ & $533 \pm 43$ & $523 \pm 24$ & $555 \pm 35$ & 0.89 & 0.92 & 0.37 \\
\hline $\mathrm{TAG}, \mathrm{mmol} / \mathrm{L}$ & $1.29 \pm 0.16$ & $1.52 \pm 0.16$ & $1.61 \pm 0.23$ & $1.61 \pm 0.24$ & 0.13 & 0.46 & 0.17 \\
\hline Cholesterol, $\mathrm{mmol} / \mathrm{L}$ & $5.9 \pm 0.3$ & $5.8 \pm 0.3$ & $6.0 \pm 0.2$ & $5.8 \pm 0.2$ & 0.13 & 0.81 & 0.49 \\
\hline HDL-c, mmol/L & $1.23 \pm 0.07$ & $1.20 \pm 0.07$ & $1.27 \pm 0.08$ & $1.29 \pm 0.09$ & 0.66 & 0.57 & 0.14 \\
\hline LDL-c, mmol/L & $4.40 \pm 0.27$ & $4.30 \pm 0.27$ & $4.45 \pm 0.22$ & $4.20 \pm 0.16$ & 0.06 & 0.94 & 0.39 \\
\hline Cholesterol/HDL-c & $5.09 \pm 0.34$ & $5.27 \pm 0.43$ & $5.16 \pm 0.42$ & $4.94 \pm 0.42$ & 0.94 & 0.82 & 0.03 \\
\hline Adiponectin, $\mu \mathrm{g} / \mathrm{mL}$ & $9.01 \pm 0.85$ & $9.64 \pm 1.05$ & $8.74 \pm 0.82$ & $8.99 \pm 0.87$ & 0.15 & 0.93 & 0.52 \\
\hline Leptin, ng/mL & $23.4 \pm 4.2$ & $22.7 \pm 4.1$ & $19.8 \pm 2.4$ & $18.2 \pm 2.4$ & 0.28 & 0.41 & 0.65 \\
\hline $\mathrm{IL}-6, \mathrm{pg} / \mathrm{mL}$ & $0.96 \pm 0.17$ & $0.83 \pm 0.11$ & $0.77 \pm 0.07$ & $0.85 \pm 0.13$ & 0.93 & 0.68 & 0.25 \\
\hline $\mathrm{IL-}-8, \mathrm{pg} / \mathrm{mL}$ & $9.25 \pm 0.61$ & $9.82 \pm 0.86$ & $9.80 \pm 0.93$ & $9.19 \pm 0.70$ & 0.98 & 0.97 & 0.26 \\
\hline TNF-a, pg/mL & $2.84 \pm 0.21$ & $3.15 \pm 0.42$ & $2.69 \pm 0.18$ & $2.63 \pm 0.15$ & 0.34 & 0.35 & 0.20 \\
\hline
\end{tabular}

Values are means \pm SEMs. Data were analyzed by using a two-factor repeated-measures ANOVA, with time $\left(\mathrm{P}_{\text {time, }}\right.$ pre, post) and treatment ( $P_{\text {treatment, }}$ PLA, EGCG+RES) as factors. For EGCG, RES and dihydro-RES, 5.45, 43.8 and $43.4 \mathrm{nmol} / \mathrm{mL}$, respectively, were detection limits. $P<0.05$ was considered significant. No baseline differences were observed (Student's t-test). In case of a significant time*treatment interaction ( $P_{\text {time }}{ }^{*}$ treatment), post-hoc analyses with Bonferroni correction were applied to identify significant within-group effects, indicated by an asterisk $\left(^{*}\right)$. Except for polyphenol concentrations, no significant within-group effects were found. EGCG+RES, epigallocatechin-3-gallate plus resveratrol; EGCG, free plasma epigallocatechin-3-gallate; FFAs, free fatty acids; HDL-c, HDL-cholesterol; HOMA-IR, homeostatic model assessment of insulin resistance; LDL-C, LDL-cholesterol; PLA, placebo; post, after intervention; pre, before intervention; RES, total plasma resveratrol; TAG, plasma triacylglycerol.

\section{EGCG+RES did not alter food intake and body composition}

Food intake, assessed by using self-reported 3-day food records, did not change after supplementation in either group (EGCG+RES: $2092 \pm 200 \mathrm{kcal} / \mathrm{d}, 45 \pm 1 \%$ of energy from carbohydrate, $39 \pm 1 \%$ of energy from fat, $15 \pm 1 \%$ of energy from protein, PLA: $1982 \mathrm{kcal} / \mathrm{d}$, $45 \pm 2 \%$ of energy from carbohydrate, $38 \pm 2 \%$ of energy from fat, $16 \pm 1 \%$ of energy from protein).

Body weight, body fat percentage and body fat distribution (upper and lower body fat mass) were not significantly altered by EGCG+RES supplementation (Table 5.3). Interestingly, however there was a tendency toward a differential effect on visceral adipose tissue mass, with a tendency toward a decrease after EGCG+RES compared with PLA $\left(\mathrm{P}_{\text {time*treatment }}=0.09\right)$. 

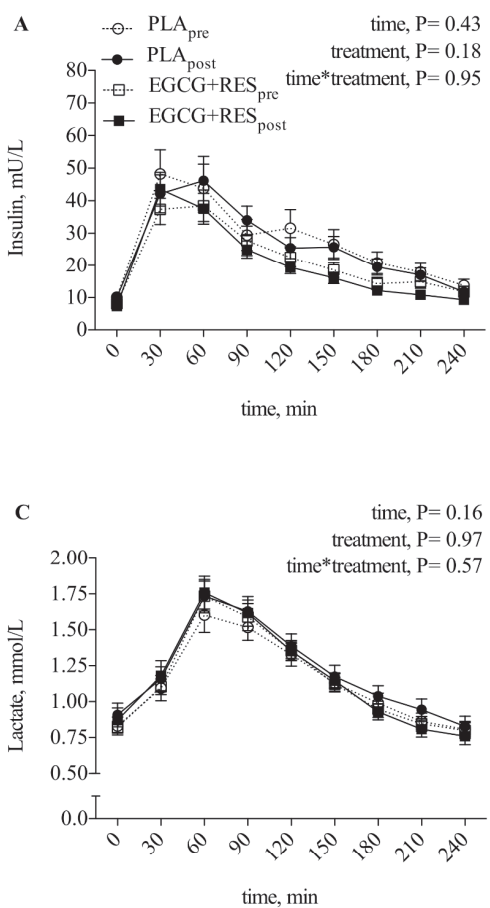

$\mathbf{E}$

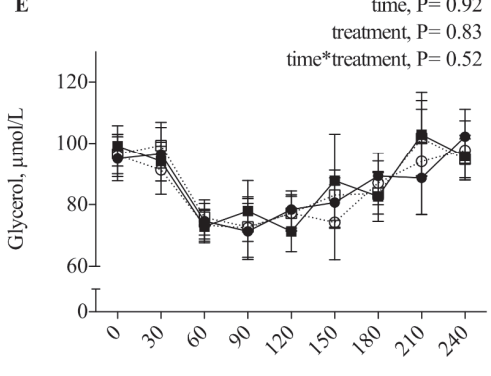

time, $\min$
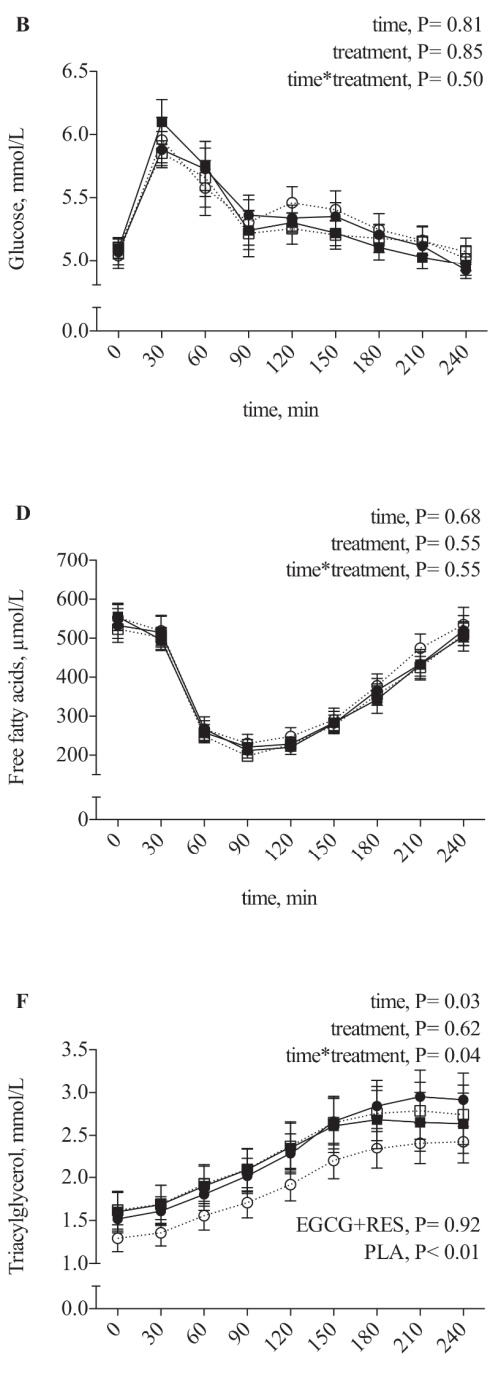

time, $\min$

\section{Figure 5.1: Fasting and postprandial insulin and metabolite concentrations before and after the intervention}

Values are means \pm SEMs $(n=38)$. Plasma insulin $(A)$ and metabolite $(B-F)$ concentrations are shown after an overnight fast (at $0 \mathrm{~min}$ ) and after a HFFM (from $0 \mathrm{~min}$ to $240 \mathrm{~min}$ ). Fasting values ( $t=0)$, AUCs during the HFMM test (calculated from $t=0-240 \mathrm{~min}$ ), and incremental AUCs were analyzed by using a two-factor repeated-measures ANOVA, with time ( $P_{\text {time, }}$ pre, post) and treatment (Ptreatment, PLA, EGCG+RES) as factors. $P<0.05$ was considered significant. No time*treatment interactions were observed on fasting concentrations and incremental AUCs. Reported P-values refer to the respective AUCs. In case of a significant time*treatment interaction ( $\left.P_{\text {time*treatment }}\right)$, post-hoc analyses with Bonferroni correction were applied to identify significant withingroup effects (EGCG+RES, P; PLA, P). EGCG+RES, epigallocatechin-3-gallate plus resveratrol; HFMM, high-fat mixed meal; PLA, placebo; post, after intervention; pre, before intervention. Dashed line, open circles: PLApre; solid line, filled circles: PLApost; Dashed line, open squares: EGCG+RESpre; solid line, filled squares: EGCG+RESpost. 
Table 5.3: Body composition before and after 12 wk supplementation with EGCG+RES or PLA

\begin{tabular}{lccccccc}
\hline & \multicolumn{2}{c}{$\begin{array}{c}\text { PLA } \\
(\mathrm{n}=20)\end{array}$} & \multicolumn{2}{c}{$\begin{array}{c}\text { EGCG+RES } \\
(\mathrm{n}=18)\end{array}$} & Time & Treatment & Time*treatment \\
\hline & Week 0 & Week 12 & Week 0 & Week 12 & $\mathrm{P}$ & $\mathrm{P}$ & $\mathrm{P}$ \\
\hline Body weight, $\mathrm{kg}$ & $88.3 \pm 2.8$ & $88.6 \pm 2.9$ & $92.4 \pm 3.6$ & $92.0 \pm 3.8$ & 0.96 & 0.46 & 0.41 \\
Body fat, kg & $26.7 \pm 1.9$ & $26.5 \pm 1.9$ & $29.4 \pm 1.4$ & $29.1 \pm 1.6$ & 0.32 & 0.36 & 0.85 \\
Body fat, \% & $30.1 \pm 1.9$ & $29.9 \pm 1.8$ & $31.9 \pm 1.4$ & $31.5 \pm 1.4$ & 0.11 & 0.55 & 0.84 \\
Lean mass, kg & $59.5 \pm 2.5$ & $59.6 \pm 2.5$ & $61.2 \pm 3.0$ & $61.1 \pm 3.2$ & 0.77 & 0.67 & 0.73 \\
Upper body fat, $\mathrm{kg}$ & $13.1 \pm 1.0$ & $12.9 \pm 1.0$ & $14.0 \pm 0.7$ & $13.8 \pm 0.7$ & 0.33 & 0.49 & 0.93 \\
Lower body fat, $\mathrm{kg}$ & $9.4 \pm 0.9$ & $9.4 \pm 0.9$ & $11.0 \pm 0.8$ & $10.8 \pm 0.9$ & 0.24 & 0.32 & 0.51 \\
VAT, kg & $0.48 \pm 0.04$ & $0.50 \pm 0.05$ & $0.44 \pm 0.05$ & $0.41 \pm 0.04$ & 0.88 & 0.39 & 0.09 \\
\hline
\end{tabular}

Values are means \pm SEMs. Data were analyzed by using a two-factor repeated-measures ANOVA, with time $\left(\mathrm{P}_{\text {time, }}\right.$ pre, post) and treatment ( $P$ treatment, PLA, EGCG+RES) as factors. $P<0.05$ was considered significant. No baseline differences were observed (Student's t-test). In case of a significant time*treatment interaction ( $\mathrm{P}_{\text {time*treatment }}$ ), post-hoc analyses with Bonferroni correction were applied to identify significant within-group effects. No significant within-groups were found. EGCG+RES, epigallocatechin-3gallate plus resveratrol; PLA, placebo; post, after intervention; pre, before intervention; VAT, visceral adipose tissue mass.

\section{EGCG+RES increased skeletal muscle oxidative capacity}

Next, we investigated whether skeletal muscle oxidative capacity increased after 12 wk EGCG+RES supplementation, because we previously showed that RES supplementation for 30 days improved mitochondrial capacity [13]. Therefore, ex vivo mitochondrial respiration with the use of different substrate combinations was determined in isolated human permeabilized skeletal muscle fibers [22].

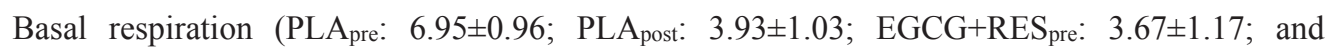
EGCG+RES post $: 4.87 \pm 1.04$ pmol O$_{2} * \mathrm{mg} \mathrm{muscle}^{-1} * \mathrm{~s}^{-1}$ ) increased by $\sim 10$-fold by the addition of ADP (state 3 respiration over basal respiration, with malate and glutamate as substrates; data not shown). This indicates that the permeabilized fibers are reactive to ADP and the mitochondrial ADP transport is intact and functioning. ADP-stimulated complex I-linked respiration with the addition of malate + glutamate was not affected by EGCG+RES supplementation (data not shown). In addition, respiration with a fatty acid substrate, assessed by input of malate + octanoylcarnitine, was not significantly affected by EGCG+RES ( $\mathrm{P}_{\text {time*treatment }}=0.19$, Figure 5.2A). Interestingly, however, we found that mitochondrial respiration upon the electron input of both complexes I and II (state 3, complex I \& II), assessed by the addition of succinate after malate + glutamate (MGS) or after malate + octanoyl-carnitine + glutamate (MGOS), increased in the EGCG+RES group compared with the PLA group after 12 wk of supplementation (state 3 respiration (MGS): $\mathrm{P}_{\text {time }} *$ treatment $=0.01, \mathrm{P}_{\mathrm{EGCG}+\mathrm{RES}}=0.01, \mathrm{P}_{\mathrm{PLA}}=0.26$; state 3 respiration (MOGS): $\mathrm{P}_{\text {time }}{ }^{*}$ treatment $=0.05, \mathrm{P}_{\mathrm{EGCG}+\mathrm{RES}}<0.01, \mathrm{P}_{\mathrm{PLA}}=0.45$, Figure 5.2B, C). Despite randomization, there was 
a difference in state 3 mitochondrial respiration at baseline between groups $\left(\mathrm{P}_{\mathrm{pre}}=0.01\right)$, which was inversely correlated to the change in this parameter over time $(\mathrm{r}=-0.57, \mathrm{P}<0.01)$. For this reason, baseline state 3 respiration was included as a covariate in the analysis.

Furthermore, maximal mitochondrial respiration, as determined by titration of the chemical uncoupler carbonyl cyanide p-trifluoromethoxyphenylhydrazone (FCCP), increased after EGCG+RES compared with PLA ( $\mathrm{P}_{\text {time }}{ }^{*}$ treatment $=0.01 ; \mathrm{P}_{\mathrm{EGCG}+\mathrm{RES}}=0.01, \mathrm{P}_{\mathrm{PLA}}=0.31$, Figure 5.2D). Mitochondrial proton leak, which was assessed by the addition of the ATP synthase inhibitor oligomycin, was not significantly affected by the intervention (state $4 \mathrm{o}, \mathrm{P}_{\text {time }} *$ treatment $=0.10$, Figure $5.2 \mathrm{E})$.

\section{EGCG+RES increased oxidative metabolism pathways at the transcriptional and translational level}

To identify pathways that may underlie the EGCG+RES-induced improvement in mitochondrial capacity, we next determined whether protein expression of OxPhos complexes in skeletal muscle was altered by EGCG+RES. We found an EGCG+RES-induced increase in complexes III

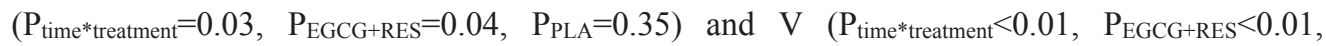
$\mathrm{P}_{\mathrm{PLA}}=0.29$ ) compared with PLA (Figure 5.2G-H). Despite randomization, there was a difference in complexes III and V at baseline between groups ( $>20 \%$ baseline difference). Adjustment for these baseline values did not alter the outcome of the analysis. On the basis of these findings, we performed microarray analysis on skeletal muscle biopsy samples (M. vastus lateralis), collected before and after $12 \mathrm{wk}$ of EGCG+RES or PLA treatment. Indeed, gene set enrichment analysis indicated that the most upregulated pathways after EGCG+RES supplementation were related to the citric acid cycle and respiratory electron transport chain, whereas pathways related to carbohydrate metabolism were upregulated in the PLA group (Figure 5.3, Supplemental Table S5.1). Taken together, these data indicate that the increased mitochondrial capacity after EGCG+RES supplementation is accompanied by changes at the transcriptional and translational levels. 

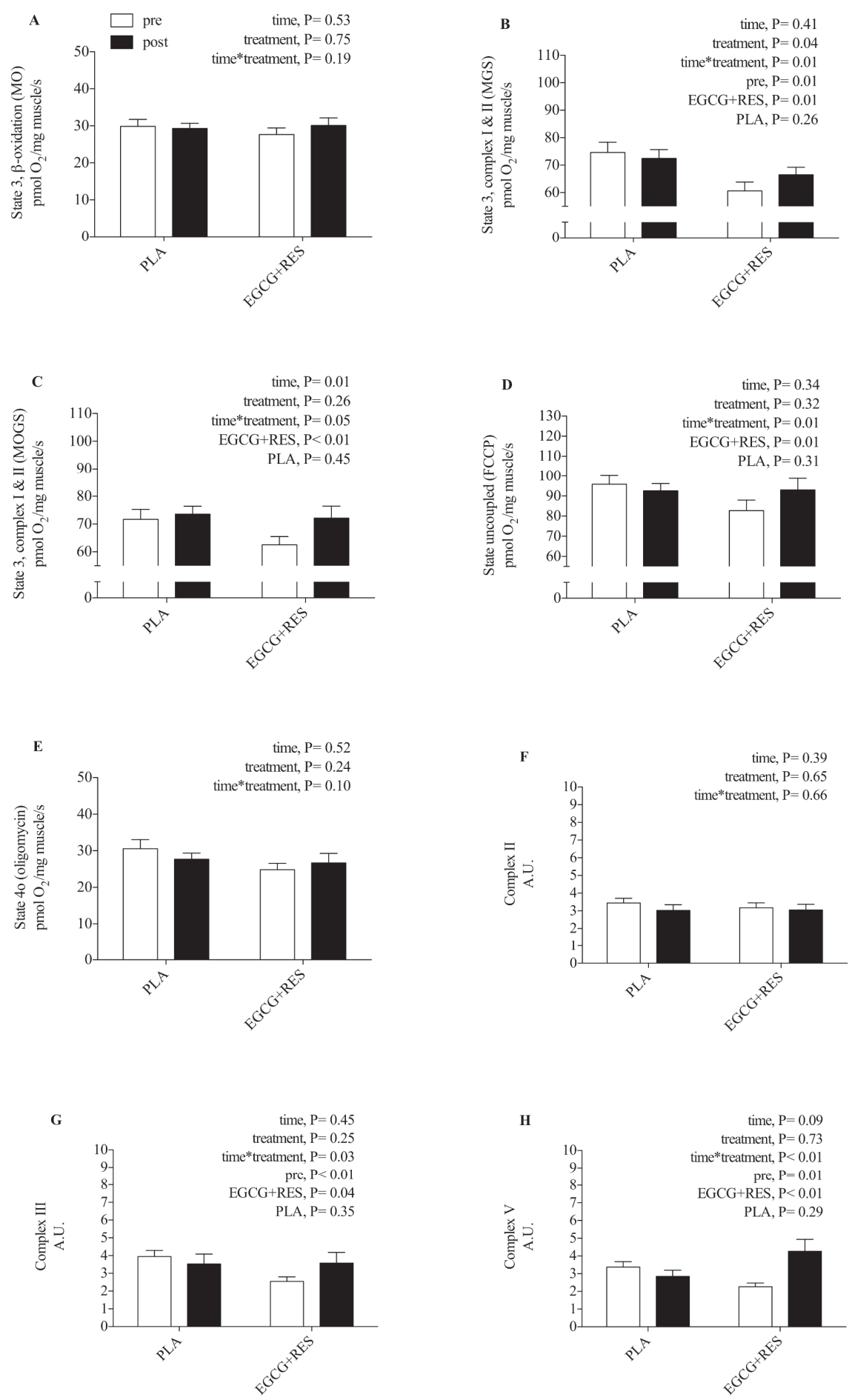
Figure 5.2: Skeletal muscle oxidative capacity and OxPhos protein content before and after the intervention

Values are means \pm SEMs $(n=29)$. Oxidative capacity $(A-E)$ was assessed by means of ex vivo respirometry on skeletal muscle (M. vastus lateralis), isolated after an overnight fast. In aliquots, protein contents of OxPhos complexes were analyzed by Western blots $(\mathrm{F}-\mathrm{H})$. Variables were analyzed by using a two-factor repeated-measures ANOVA, with time ( $\mathrm{P}_{\text {time, }}$ pre, post) and treatment ( $P_{\text {treatment, }}$ PLA, EGCG+RES) as factors. $P<0.05$ was considered significant. Significant baseline differences were identified by Student's unpaired t-test $\left(P_{\text {pre }}\right)$; and in case of a significant time*treatment interaction $\left(P_{\text {timetreatment }}\right)$, post-hoc analyses with Bonferroni correction were applied to identify significant within-group effects (EGCG+RES, P; PLA, P). AU: arbitrary units; EGCG+RES, epigallocatechin-3-gallate plus resveratrol; FCCP: carbonyl cyanide p-trifluoromethoxyphenylhydrazone; G: glutamate; M: malate; O: octanoyl-carnitine; OxPhos, oxidative phosphorylation; PLA, placebo; post, after intervention; pre, before intervention; S: succinate. White bars: pre; black bars: post intervention.

\section{EGCG+RES affects fat oxidation without changes in $E E$}

Next, we investigated whether the improved skeletal muscle oxidative capacity after $12 \mathrm{wk}$ of EGCG+RES supplementation translated into whole-body effects on fasting substrate oxidation. Indeed, EGCG+RES supplementation significantly affected fasting substrate oxidation, as reflected by no change in fasting RQ after EGCG+RES and a significant increase in the PLA group

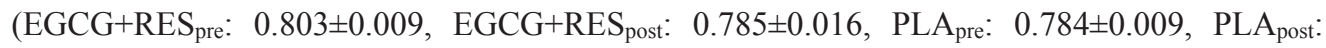
$0.821 \pm 0.015, \mathrm{P}_{\text {time }} *$ treatment $=0.03, \mathrm{P}_{\mathrm{EGCG}+\mathrm{RES}}=0.31, \mathrm{P}_{\mathrm{PLA}}=0.04$; Figure $\left.5.4 \mathrm{~A}\right)$, without effects on $\mathrm{EE}$ (Figure 5.4B). More specifically, an increase in fasting carbohydrate oxidation was observed after PLA, but not after EGCG+RES ( $\left.\mathrm{P}_{\text {time*treatment }}=0.05, \mathrm{P}_{\mathrm{EGCG}+\mathrm{RES}}=0.44, \mathrm{P}_{\mathrm{PLA}}=0.04\right)$ supplementation . Furthermore, EGCG+RES did not change fasting fat oxidation, whereas there was a significant decrease in the PLA group after 12 wk of supplementation as compared with week 0 $\left(\mathrm{P}_{\text {time*treatment }}=0.06, \mathrm{P}_{\mathrm{EGCG}+\mathrm{RES}}=0.46, \mathrm{P}_{\mathrm{PLA}}=0.05\right.$, Figures $\left.5.4 \mathrm{C}, \mathrm{D}\right)$.

\section{EGCG+RES affects TAG concentrations but did not change postprandial responses of substrate oxidation and local lipolysis}

To examine EGCG+RES-induced effects on postprandial substrate utilization and EE, we performed a HFMM test (625 kcal, 61\% of energy from fat). More specifically, we determined whole-body, AT and skeletal muscle substrate metabolism in the postprandial state by combining plasma blood sampling, indirect calorimetry and microdialysis.

The effects of 12 wk EGCG+RES supplementation on substrate oxidation in the absence of changes in $\mathrm{EE}$ in the fasting state were maintained throughout the postprandial period (Figure 5.4E-H). More specifically, postprandial fat oxidation (AUC) was reduced in the PLA group in week 12 compared with week $0\left(\mathrm{P}_{\mathrm{PLA}}=0.03\right)$, whereas no significant effect of EGCG+RES was observed $\left(\mathrm{P}_{\mathrm{EGCG}+\mathrm{RES}}=0.38\right.$, Figure 5.4H). In addition, the intervention-induced changes in EGCG, RES, and dihydro-RES plasma concentrations did not significantly correlate with EGCG+RESinduced effects on fat oxidation (data not shown). 
Dietary polyphenols and insulin sensitivity

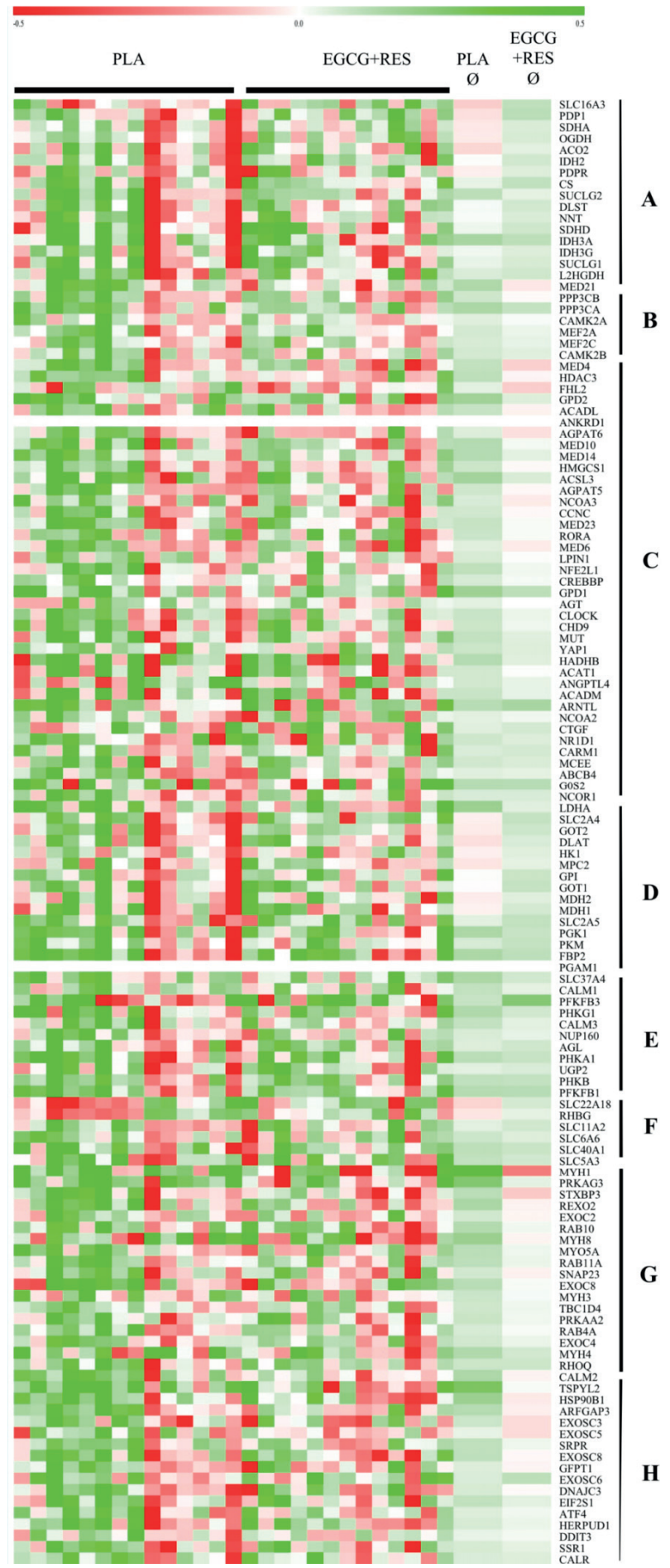

Figure 5.3: Skeletal muscle gene set enrichment analyses before and after the intervention

Heatmap of pathways related to metabolic function from gene set enrichment analyses on skeletal muscle (M. vastus lateralis) $(n=27)$ are shown. Signal-log-ratios are presented per subject and as means per group. Genes in the heatmap represent significantly contributing genes to most affected pathways by EGCG+RES or PLA supplementation (false discovery rate, $q$-value $<0.2)$. Colors reflect the signal-log-ratios per subject and group (Ø), with red being down-regulated and green being up-regulated comparing post- with premeasurement. EGCG+RES, epigallocatechin3-gallate plus resveratrol; PLA, placebo; post, after intervention; pre, before intervention. A list of definitions of gene and protein names used in this figure is included in the Supplemental Material

A: WP2766: THE. CITRIC. ACID. TCA. CYCLE. AND. RESPIRATORY. ELECTRON. TRANSPORT;

B: BIOC_PGC1A PATHWAY;

C: FATTY. ACID. TRIACYLGLYCEROL. AND. KETONE. BODY. METABOLISM; D: WP534: GLYCOLYSIS. AND.

GLUCONEOGENESIS;

E: WP1848: METABOLISM. OF. CARBOHYDRATES;

F: WP1935: TRANSPORT. OF. GLUCOSE. AND. OTHER. SUGARS. BILE. SALTS. AND. ORGANIC. ACIDS. METAL. IONS. AND.

AMINE. COMPOUNDS;

G: TRANSLOCATION. OF. GLUT4. TO. THE. PLASMA. MEMBRANE;

H: UNFOLDED.PROTEIN. RESPONSE.UPR. 
The postprandial responses after meal intake (incremental AUC) of RQ, EE, carbohydrate, and fat oxidation as well as the postprandial responses of plasma metabolites and insulin were not significantly affected by the intervention (Figure 5.4I-L and Figure 5.1A-F, respectively). Importantly, however, plasma TAG concentrations during the HFMM test were significantly increased in the PLA group after $12 \mathrm{wk}$, but not in the EGCG+RES group (PLA pre: AUC $=440 \pm 56$, PLA $_{\text {post }}: \quad \mathrm{AUC}=531 \pm 62, \quad \mathrm{EGCG}+\mathrm{RES}_{\text {pre }}: \quad \mathrm{AUC}=527 \pm 59, \quad \mathrm{EGCG}+\mathrm{RES}_{\text {post: }}: \quad \mathrm{AUC}=530 \pm 66$ $\mathrm{mmol}^{*} 4 \mathrm{~h} / \mathrm{L}, \mathrm{P}_{\text {time }} *$ treatment $=0.04, \mathrm{P}_{\mathrm{EGCG}+\mathrm{RES}}=0.92, \mathrm{P}_{\mathrm{PLA}}<0.01$; Figure 5.1F).

Skeletal muscle and abdominal subcutaneous adipose tissue substrate metabolism and blood flow were investigated in men with the use of microdialysis. AT and skeletal muscle ethanol outflow/inflow ratios, which indicate local blood flow, were not significantly affected by the intervention (Supplemental Figure S5.1). Interstitial glycerol concentrations, which reflect local lipolysis, were also not affected by EGCG+RES (Supplemental Figure S5.2). Furthermore, no effects of EGCG+RES supplementation were observed on interstitial glucose, pyruvate, and lactate concentrations.

\section{EGCG+RES did not affect intramuscular lipid content and composition}

Alterations in fat oxidation may contribute to changes in intramyocellular lipid metabolites and, consequently, insulin sensitivity [2]. EGCG+RES supplementation for $12 \mathrm{wk}$ did not alter total lipid content (TAG and DAG) or saturation of the TAG and DAG fractions (Supplemental Table S5.2).

\section{EGCG+RES did not affect tissue-specific insulin sensitivity}

Finally, we investigated whether the improved mitochondrial capacity and whole-body fat oxidation resulted in improved insulin sensitivity after EGCG+RES supplementation. We found no changes in peripheral, hepatic or AT insulin sensitivity after EGCG+RES supplementation compared with PLA supplementation (Table 5.4), which is in agreement with unchanged fasting and postprandial plasma glucose and insulin concentrations. More specifically, the $\mathrm{R}_{\mathrm{d}}$ and EGP under fasting and insulin-stimulated conditions were not significantly affected following $12 \mathrm{wk}$ of EGCG+RES supplementation (Table 5.4). Similar results were found when values were expressed as changes relative to fasting conditions (Table 5.4). Interestingly, EGCG+RES increased fasting NOGD $\left(\mathrm{P}_{\text {time* }}{ }^{\text {treatment }}=0.10, \mathrm{P}_{\mathrm{EGCG}+\mathrm{RES}}=0.01, \mathrm{P}_{\mathrm{PLA}}=0.91\right)$. Furthermore, EGCG+RES did not improve insulin-mediated suppression of plasma FFA, indicating that adipose tissue insulin sensitivity was also not affected by EGCG+RES supplementation $\left(\mathrm{P}_{\text {time*treatment }}=0.63\right.$, Table 5.4). 

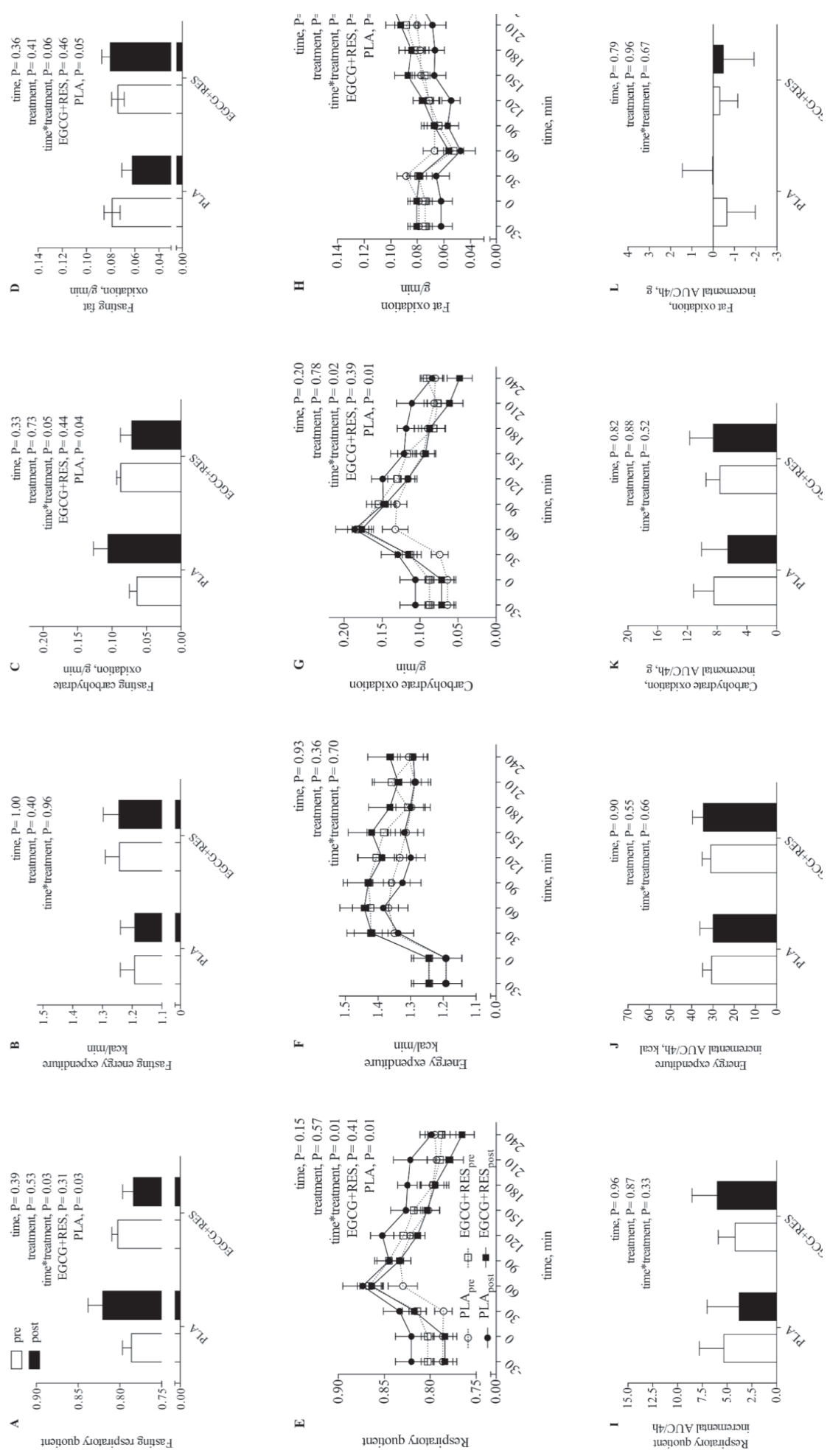
Figure 5.4: Fasting and postprandial whole-body substrate oxidation and EE before and after the intervention

Values are means \pm SEMs $(n=31)$. Indirect calorimetry was performed during fasting conditions and for $4 \mathrm{~h}$ after intake of a liquid HFMM. Mean $\mathrm{O}_{2}$-consumption and $\mathrm{CO}_{2}$-production over 20 min were used for calculations. Substrate oxidation (A, C-D) and EE (B) were measured after an overnight fast (from -30 to $0 \mathrm{~min}$ ) and after a HFMM (from 0 to $240 \mathrm{~min}$ ). Fasting values (A-D), AUCs during the HFMM test (calculated from $t=0$ to $240 \mathrm{~min}$; E-H), and incremental AUCs (I-L) were analyzed using a two-factor repeated-measures ANOVA, with time $\left(P_{\text {time }}, p r e, p o s t\right)$ and treatment $\left(P_{\text {treatment, }} P L A, E G C G+R E S\right)$ as factors. $P<0.05$ was considered significant. In case of a significant time ${ }^{*}$ treatment interaction ( $\left.P_{\text {timetreatment }}\right)$, post-hoc analyses with Bonferroni correction were applied to identify significant within-group effects (EGCG+RES, P; PLA, $P$ ). EGCG+RES, epigallocatechin-3-gallate plus resveratrol; EE, energy expenditure; PLA, placebo; post, after intervention; pre, before intervention. Figures A-D and I-L: White bars: pre; black bars: post; Figures E-H: Dashed line, open circles: PLApre; solid line, filled circles: PLApost; Dashed line, open squares: EGCG+RES pre; solid line, filled squares: $E G C G+R E S_{\text {post. }}$

Table 5.4: Whole-body substrate kinetics before and after $12 \mathrm{wk}$ of supplementation with EGCG+RES or PLA

\begin{tabular}{|c|c|c|c|c|c|c|c|}
\hline & \multicolumn{2}{|c|}{$\begin{array}{c}\text { PLA } \\
(n=19)\end{array}$} & \multicolumn{2}{|c|}{$\begin{array}{c}\text { EGCG+RES } \\
(n=17)\end{array}$} & \multirow{2}{*}{$\frac{\text { Time }}{\mathrm{P}}$} & \multirow{2}{*}{$\begin{array}{c}\text { Treatment } \\
\mathrm{P}\end{array}$} & \multirow{2}{*}{$\frac{\begin{array}{c}\text { Time }^{*} \\
\text { treatment }\end{array}}{\mathrm{P}}$} \\
\hline & Week 0 & Week 12 & Week 0 & Week 12 & & & \\
\hline \multicolumn{8}{|l|}{$\mathrm{R}_{\mathrm{d}}, \mu \mathrm{mol} /\left(\mathrm{kg}^{*} \min \right)$} \\
\hline Fasting & $10.6 \pm 0.3$ & $10.7 \pm 0.3$ & $10.4 \pm 0.2$ & $10.9 \pm 0.3$ & 0.25 & 0.91 & 0.19 \\
\hline Clamp & $31.2 \pm 2.3$ & $32.5 \pm 2.3$ & $34.2 \pm 2.8$ & $35.8 \pm 3.3$ & 0.25 & 0.38 & 0.89 \\
\hline Stimulation, $\Delta$ & $20.6 \pm 2.2$ & $21.5 \pm 2.3$ & $24.3 \pm 3.0$ & $25.0 \pm 3.2$ & 0.35 & 0.27 & 0.84 \\
\hline \multicolumn{8}{|c|}{$\mathrm{NOGD}, \mu \mathrm{mol} /\left(\mathrm{kg}^{*} \min \right)$} \\
\hline Fasting & $6.1 \pm 0.7$ & $6.2 \pm 0.6$ & $5.7 \pm 0.6$ & $7.9 \pm 0.7^{\star}$ & 0.05 & 0.41 & 0.10 \\
\hline Clamp & $20.5 \pm 2.4$ & $23.4 \pm 1.9$ & $23.0 \pm 2.5$ & $25.3 \pm 3.1$ & 0.16 & 0.73 & 0.95 \\
\hline stimulation, $(\Delta)$ & $14.4 \pm 2.1$ & $17.2 \pm 2.1$ & $17.5 \pm 2.7$ & $17.4 \pm 2.6$ & 0.54 & 0.76 & 0.44 \\
\hline \multicolumn{8}{|l|}{ EGP } \\
\hline $\begin{array}{l}\text { Fasting, } \\
\mu \mathrm{mol} /\left(\mathrm{kg}^{*} \mathrm{~min}\right) \\
\text { Clamp, }\end{array}$ & $10.4 \pm 0.3$ & $10.4 \pm 0.3$ & $10.1 \pm 0.2$ & $10.7 \pm 0.3$ & 0.25 & 0.91 & 0.19 \\
\hline$\mu \mathrm{mol} /\left(\mathrm{kg}^{*} \min \right)$ & $4.0 \pm 0.6$ & $4.2 \pm 0.6$ & $4.6 \pm 0.5$ & $4.6 \pm 0.5$ & 0.78 & 0.57 & 0.98 \\
\hline Suppression, \% & $60.9 \pm 4.9$ & $58.6 \pm 4.9$ & $54.1 \pm 5.1$ & $56.3 \pm 4.7$ & 0.98 & 0.57 & 0.74 \\
\hline \multicolumn{8}{|l|}{ Plasma FFAs } \\
\hline Fasting, $\mu \mathrm{mol} / \mathrm{L}$ & $545 \pm 40$ & $547 \pm 44$ & $537 \pm 42$ & $543 \pm 47$ & 0.88 & 0.91 & 0.94 \\
\hline Clamp, $\mu \mathrm{mol} / \mathrm{L}$ & $196 \pm 25$ & $210 \pm 22$ & $189 \pm 26$ & $203 \pm 23$ & 0.17 & 0.83 & 0.94 \\
\hline Suppression, \% & $57.7 \pm 6.6$ & $57.1 \pm 5.2$ & $63.4 \pm 7.0$ & $59.0 \pm 5.5$ & 0.52 & 0.62 & 0.63 \\
\hline \multicolumn{8}{|c|}{ Substrate oxidation } \\
\hline $\mathrm{RQ}, \Delta$ & $0.09 \pm 0.01$ & $0.08 \pm 0.02$ & $0.10 \pm 0.01$ & $0.11 \pm 0.01$ & 0.96 & 0.30 & 0.31 \\
\hline $\mathrm{CHO}, \Delta \mathrm{g} / \mathrm{min}$ & $0.10 \pm 0.01$ & $0.09 \pm 0.02$ & $0.11 \pm 0.01$ & $0.12 \pm 0.02$ & 0.93 & 0.27 & 0.54 \\
\hline Fat, \% & $55.5 \pm 7.6$ & $54.3 \pm 9.1$ & $46.7 \pm 6.9$ & $48.4 \pm 8.3$ & 0.95 & 0.47 & 0.81 \\
\hline
\end{tabular}

Values are means \pm SEMs. Data were analyzed by using a two-factor repeated-measures ANOVA, with time ( $P_{\text {time, }}$ pre, post) and treatment ( $\left.P_{\text {treatment, }} P L A, E G C G+R E S\right)$ as factors. $P<0.05$ was considered significant. No baseline differences were observed (Student's t-test). In case of a significant time*treatment interaction ( $\left.P_{\text {timetreatment }}\right)$, post-hoc analyses with Bonferroni correction were applied to identify significant within-group effects, which are indicated by asterisk $\left({ }^{*}\right)$. $\mathrm{CHO}$, carbohydrate oxidation; EGCG+RES, epigallocatechin-3-gallate plus resveratrol; EGP, endogenous glucose production; NOGD, non-oxidative glucose disposal; PLA, placebo, post, after intervention; pre, before intervention; $R_{d}$, rate of disappearance; $R Q$, respiratory quotient; $\Delta$ absolute difference between fasting and insulin-stimulated conditions. 


\section{Discussion}

Combining polyphenols with distinct mechanisms of action may lead to additional or synergistic beneficial metabolic effects compared with single-polyphenol supplementation [19, 21]. Here, we show that after 12 weeks, combined EGCG+RES supplementation preserved fasting and postprandial fat oxidation as compared with PLA. This coincided with increased skeletal muscle oxidative capacity in the EGCG+RES group. In addition, EGCG+RES supplementation attenuated the increase in TAG concentrations compared with PLA, and the reduction in visceral adipose tissue mass tended to be higher with EGCG+RES than with PLA. These beneficial metabolic effects, however, did not translate into improved peripheral, hepatic and AT insulin sensitivity in overweight men and women. Because the RES supplement is composed of Polygonum cuspidatum extract, we cannot exclude metabolic effects of other components (piceid (=RES-glucoside) and emodin [28]).

We previously showed that 3 days of EGCG+RES supplementation increased EE [20]. The present study showed that this increase is not maintained after 12 wk supplementation. Furthermore, consistent with unchanged food intake, we found that body weight and body composition were not affected by longer-term EGCG+RES supplementation. These findings are in line with most [13, $14,17,18,29,30]$ but not all [15] previous studies in humans. Although no significant effects on total fat mass were apparent, we found that EGCG+RES tended to decrease visceral adipose tissue mass by $\sim 11 \%$ compared with PLA. Likewise, EGCG and RES have reduced visceral adipose tissue in animals [31, 32]. Because visceral adipose tissue is known to be detrimental to metabolic health [33], its reduction may be of physiological importance in the long term.

Interestingly, we showed that combined EGCG+RES supplementation significantly affected fasting substrate oxidation compared with PLA (Figure 5.4). EGCG+RES supplementation preserved fat oxidation, whereas fat oxidation significantly declined in the PLA group. This effect was maintained during postprandial conditions. In agreement with our findings, previous studies in rodents showed that polyphenols may stimulate fat oxidation under fasting conditions $[9,31$, 34]. The compensatory increase in carbohydrate oxidation that we found in the PLA group was not accompanied by alterations in whole-body glucose disposal. Rather, we found that EGCG+RES may diminish glycolysis and increase glycogen storage [35], as indicated by the increased fasting NOGD ( 40\%, $\left.\mathrm{P}_{\mathrm{EGCG}+\mathrm{RES}}=0.01\right)$. Importantly, the differentially affected fat oxidation in the present study is neither driven by an increased lipid supply toward skeletal muscle nor by a significant reduction in muscle lipid stores (TAG and DAG content). Indeed, it was previously shown that a shift in fasting fat oxidation can occur independently of changes in 
intramuscular lipid content, EE, adiposity or insulin sensitivity [36, 37]. Alternatively, a higher oxidative capacity of skeletal muscle mitochondria was found to be predictive of a lower fasting respiratory exchange ratio or, in other words, a greater relative reliance on lipids instead of carbohydrate as a fuel source during fasting conditions [37-39]. Interestingly, we found that $12 \mathrm{wk}$ EGCG+RES supplementation increased skeletal muscle oxidative capacity in permeabilized muscle fibers. This was accompanied by an increased muscle protein content of OxPhos complexes and an upregulation of mitochondrial pathways (citric acid cycle and respiratory electron transport chain, Figure 5.2 and 5.3). In line with these findings, we and others previously showed that RES increased mitochondrial capacity by activating the AMPK-SIRT-PGC1 $\alpha$ pathway $[7,8,13]$. It has been suggested that EGCG increases $\beta$-adrenergic stimulation by norepinephrine due to catechol-O-methyltransferase inhibition [10, 40]. However, in our previous study we did not observe altered norepinephrine concentrations after EGCG+RES supplementation [20]. Finally, reduced oxidative stress, indicated by a reduced unfolded protein response pathway [41], might have contributed to the observed improvement of skeletal muscle oxidative capacity [42-44] or vice versa [45].

An increased mitochondrial capacity might reflect an improved metabolic risk profile, because increased mitochondrial capacity has been associated with higher insulin sensitivity in several cross-sectional studies in men [37-39, 46]. Therefore, we hypothesized that the improvements in lipid metabolism may lead to increased insulin sensitivity after 12 wk of EGCG+RES supplementation. However, we did not find a significant change in peripheral, hepatic, or AT insulin sensitivity. To date, only three human clinical trials with RES, but not a single study with EGCG, examined the effects of polyphenol supplementation on peripheral and hepatic insulin sensitivity by using the gold standard hyperinsulinemic-euglycemic clamp. In accordance with our findings, these studies did not show significant changes in peripheral [17, 18, 29] and hepatic [18] insulin sensitivity in lean [18] and obese [17] patients or in patients with non-alcoholic fatty-liver disease [29] after supplementation with RES for 4, 8 or 12 weeks, respectively. Importantly, in these studies, mitochondrial capacity was not assessed [29] or did not improve [17, 18], on the basis of gene expression and protein abundance of related enzymes. Therefore, the present study is the first, to our knowledge, to show that combined EGCG+RES supplementation has beneficial effects on skeletal muscle oxidative capacity, although this was not accompanied by increased peripheral, hepatic, or AT insulin sensitivity in overweight and obese men and women.

In line with these findings, the insulin-mediated stimulation of carbohydrate oxidation and suppression of lipid oxidation were not affected by EGCG+RES supplementation after meal ingestion or during the hyperinsulinemic-euglycemic clamp. Previously, we showed that single 
supplementation of RES for 4 weeks and combined EGCG+RES supplementation for 3 days improved metabolic flexibility in humans $[13,20]$, as shown by a more pronounced suppression of postprandial fat oxidation. The apparent discrepancy between the present and earlier studies may be explained by the duration of supplementation. EGCG+RES supplementation had no effect on postprandial glucose, insulin and FFA concentrations or local interstitial glucose and glycerol concentrations. This is in agreement with other placebo-controlled trials in Caucasians [13, 19], whereas sex-specific analyses may show metabolic effects [14].

Strikingly, we found that EGCG+RES supplementation for 12 weeks attenuated the increase in plasma TAG during the HFMM test that was seen in the PLA group. Previous studies in humans $[13,14]$ and rodents $[9,31,47]$ also showed reduced plasma TAG after EGCG or RES supplementation. This might, at least in part, be explained by a reduced intestinal lipid uptake, an improved buffering capacity of dietary TAGs in adipose tissue, or increased peripheral (e.g. muscle and liver) TAG extraction. In accordance, a reduced intestinal lipid uptake after EGCG supplementation in rodents has been suggested [9], although this could not be confirmed in humans [30]. In addition, EGCG+RES supplementation may have affected hepatic lipid clearance, storage, and oxidation, as indicated by several animal studies [47-49].

In the present study, we analyzed EGCG, RES and dihydro-RES plasma concentrations before and after intervention. In future studies, a more detailed characterization of polyphenol metabolites might provide additional insight into polyphenol-induced effects on oxidative metabolism, because polyphenolic metabolites may exert distinct effects on peripheral tissues [50, 51]. Furthermore, due to the extensive phenotyping in the current controlled trial, multiple statistical comparisons were made, which may increase the likelihood of obtaining false-positive results. Nevertheless, the fact that our major findings on variables of mitochondrial oxidative capacity and proteins in oxidative phosphorylation and fat oxidation all point toward the same direction of an improvement with EGCG+RES compared with PLA makes it highly unlikely that these are chance findings. Third, in this randomized trial, the statistical plan included a completers analysis taking a drop-out rate of $20 \%$ into account, which was higher than the actual drop-out rate. The drop-out rate was similar in both groups $(10 \% ; n=2)$ and was therefore assumed to be randomly distributed. An intention-to-treat analysis would therefore make the reported physiologic effects on oxidative metabolism and fat oxidation only slightly less pronounced.

In conclusion, the present study showed that $12 \mathrm{wk}$ of EGCG+RES supplementation improved skeletal muscle oxidative capacity, preserved fasting and postprandial fat oxidation, and prevented an increase in TAG concentrations compared with PLA. These putative beneficial metabolic effects did not translate into improved peripheral, hepatic, or adipose tissue insulin sensitivity after 
12 weeks. Importantly, the improved mitochondrial capacity and fat oxidation may improve physical conditions [52] and play a role in the prevention of weight gain [53] and the worsening of insulin resistance [4] in the long term.

\section{Abbreviations}

$\begin{array}{ll}\text { AMPK } & \text { AMP activated kinase } \\ \text { CID } & \text { Clinical investigation day } \\ \text { DAG } & \text { Diacylglycerol } \\ \text { EGCG } & \text { Epigallocatechin-3-gallate } \\ \text { EE } & \text { Energy expenditure } \\ \text { EGP } & \text { Endogenous glucose production } \\ \text { FCCP } & \text { Carbonyl Cyanide p-Trifluoromethoxyphenylhydrazone } \\ \text { FFA } & \text { Free fatty acids } \\ \text { HFMM } & \text { High-fat mixed meal } \\ \text { NOGD } & \text { Non-oxidative glucose disposal } \\ \text { OxPhos } & \text { Oxidative phosphorylation } \\ \text { PGC1 } \alpha & \text { Peroxisome proliferator-activated receptor gamma coactivator 1- } \alpha \\ \text { PLA } & \text { Placebo } \\ \text { RES } & \text { Resveratrol } \\ \text { Rd } & \text { Rate of disappearance } \\ \text { RQ } & \text { Respiratory quotient } \\ \text { T2DM } & \text { Type 2 diabetes mellitus } \\ \text { TAG } & \text { Triacylglycerol }\end{array}$

\section{Acknowledgements}

The authors would like to thank the study participants, as well as Jos Stegen, Wendy Sluijsmans, Yvonne Essers, Kirsten van der Beek, Gabby Hul, Hasibe Aydeniz, Nicole Hoebers, Angelika Friedel, Christine Toepfer, Esther Kornips, Gert Schaart, Stephane Etheve and Alla Fischer for excellent technical support. None of the authors have any conflicts of interest to declare. 


\section{References}

1. Ng M, Fleming T, Robinson M, Thomson B, Graetz N, Margono C, Mullany EC, Biryukov S, Abbafati C, Abera SF, et al. Global, regional, and national prevalence of overweight and obesity in children and adults during 1980-2013: a systematic analysis for the Global Burden of Disease Study 2013. Lancet 2014;384(9945):766-81.

2. Shulman GI. Ectopic Fat in Insulin Resistance, Dyslipidemia, and Cardiometabolic Disease. N Engl J Med 2014;371(12):1131-41.

3. Goossens GH. The role of adipose tissue dysfunction in the pathogenesis of obesity-related insulin resistance. Physiol Behav 2008;94(2):206-18.

4. Szendroedi J, Phielix E, Roden M. The role of mitochondria in insulin resistance and type 2 diabetes mellitus. Nat Rev Endocrinol 2012;8(2):92-103.

5. van Hees AM, Jans A, Hul GB, Roche HM, Saris WH, Blaak EE. Skeletal muscle fatty acid handling in insulin resistant men. Obesity (Silver Spring) 2011;19(7):1350-9.

6. Roumen C, Corpeleijn E, Feskens EJ, Mensink M, Saris WH, Blaak EE. Impact of 3-year lifestyle intervention on postprandial glucose metabolism: the SLIM study. Diabet Med 2008;25(5):597-605.

7. Baur JA, Pearson KJ, Price NL, Jamieson HA, Lerin C, Kalra A, Prabhu VV, Allard JS, Lopez-Lluch G, Lewis K, et al. Resveratrol improves health and survival of mice on a high-calorie diet. Nature 2006;444(7117):337-42.

8. Lagouge M, Argmann C, Gerhart-Hines Z, Meziane H, Lerin C, Daussin F, Messadeq N, Milne J, Lambert P, Elliott $\mathrm{P}$, et al. Resveratrol improves mitochondrial function and protects against metabolic disease by activating SIRT1 and PGC-1alpha. Cell 2006;127(6):1109-22.

9. Klaus S, Pultz S, Thone-Reineke C, Wolfram S. Epigallocatechin gallate attenuates diet-induced obesity in mice by decreasing energy absorption and increasing fat oxidation. Int J Obes (Lond) 2005;29(6):615-23.

10. Lu H, Meng X, Yang CS. Enzymology of methylation of tea catechins and inhibition of catechol-Omethyltransferase by (-)-epigallocatechin gallate. Drug Metab Dispos 2003;31(5):572-9.

11. Collins QF, Liu HY, Pi J, Liu Z, Quon MJ, Cao W. Epigallocatechin-3-gallate (EGCG), a green tea polyphenol, suppresses hepatic gluconeogenesis through 5'-AMP-activated protein kinase. J Biol Chem 2007;282(41):30143-9.

12. Bogdanski P, Suliburska J, Szulinska M, Stepien M, Pupek-Musialik D, Jablecka A. Green tea extract reduces blood pressure, inflammatory biomarkers, and oxidative stress and improves parameters associated with insulin resistance in obese, hypertensive patients. Nutr Res 2012;32(6):421-7.

13. Timmers S, Konings E, Bilet L, Houtkooper RH, van de Weijer T, Goossens GH, Hoeks J, van der Krieken S, Ryu D, Kersten S, et al. Calorie restriction-like effects of 30 days of resveratrol supplementation on energy metabolism and metabolic profile in obese humans. Cell Metab 2011;14(5):612-22.

14. Mahler A, Steiniger J, Bock M, Klug L, Parreidt N, Lorenz M, Zimmermann BF, Krannich A, Paul F, Boschmann M. Metabolic response to epigallocatechin-3-gallate in relapsing-remitting multiple sclerosis: a randomized clinical trial. Am J Clin Nutr 2015;101(3):487-95.

15. Brown AL, Lane J, Holyoak C, Nicol B, Mayes AE, Dadd T. Health effects of green tea catechins in overweight and obese men: a randomised controlled cross-over trial. Br J Nutr 2011;106(12):18809 .

16. Mendez-Del Villar M, Gonzalez-Ortiz M, Martinez-Abundis E, Perez-Rubio KG, Lizarraga-Valdez R. Effect of Resveratrol Administration on Metabolic Syndrome, Insulin Sensitivity, and Insulin Secretion. Metab Syndr Relat Disord 2014.

17. Poulsen MM, Vestergaard PF, Clasen BF, Radko Y, Christensen LP, Stodkilde-Jorgensen H, Moller $\mathrm{N}$, Jessen N, Pedersen SB, Jorgensen JO. High-dose resveratrol supplementation in obese men: an investigator-initiated, randomized, placebo-controlled clinical trial of substrate metabolism, insulin sensitivity, and body composition. Diabetes 2013;62(4):1186-95.

18. Yoshino J, Conte C, Fontana L, Mittendorfer B, Imai S, Schechtman KB, Gu C, Kunz I, Rossi Fanelli F, Patterson BW, et al. Resveratrol supplementation does not improve metabolic function in nonobese women with normal glucose tolerance. Cell Metab 2012;16(5):658-64.

19. Brown AL, Lane J, Coverly J, Stocks J, Jackson S, Stephen A, Bluck L, Coward A, Hendrickx H. Effects of dietary supplementation with the green tea polyphenol epigallocatechin-3-gallate on insulin 
resistance and associated metabolic risk factors: randomized controlled trial. $\mathrm{Br} \mathrm{J}$ Nutr 2009;101(6):886-94.

20. Most J, Goossens GH, Jocken JW, Blaak EE. Short-term supplementation with a specific combination of dietary polyphenols increases energy expenditure and alters substrate metabolism in overweight subjects. Int J Obes (Lond) 2014;38(5):698-706.

21. Van Can J, Van Dijk J-W, Goossens G, Jocken J, Hospers J, Blaak E. A 3 day EGCG supplementation reduced lactate in skeletal muscle in overweight subjects. Obesity Reviews 2010;11(1):206.

22. Phielix E, Schrauwen-Hinderling VB, Mensink M, Lenaers E, Meex R, Hoeks J, Kooi ME, MoonenKornips E, Sels JP, Hesselink MK, et al. Lower intrinsic ADP-stimulated mitochondrial respiration underlies in vivo mitochondrial dysfunction in muscle of male type 2 diabetic patients. Diabetes 2008;57(11):2943-9.

23. Goossens GH, Blaak EE, Saris WH, van Baak MA. Angiotensin II-induced effects on adipose and skeletal muscle tissue blood flow and lipolysis in normal-weight and obese subjects. J Clin Endocrinol Metab 2004;89(6):2690-6.

24. Weir JB. New methods for calculating metabolic rate with special reference to protein metabolism. J Physiol 1949;109(1-2):1-9.

25. Frayn KN. Calculation of substrate oxidation rates in vivo from gaseous exchange. J Appl Physiol Respir Environ Exerc Physiol 1983;55(2):628-34.

26. Friedewald WT, Levy RI, Fredrickson DS. Estimation of the concentration of low-density lipoprotein cholesterol in plasma, without use of the preparative ultracentrifuge. Clin Chem 1972;18(6):499-502.

27. Stampfer MJ, Sacks FM, Salvini S, Willett WC, Hennekens CH. A prospective study of cholesterol, apolipoproteins, and the risk of myocardial infarction. N Engl J Med 1991;325(6):373-81.

28. Kuo CH, Chen BY, Liu YC, Chang CMJ, Deng TS, Chen JH, Shieh CJ. Optimized UltrasoundAssisted Extraction of Phenolic Compounds from Polygonum cuspidatum. Molecules 2014;19(1):67-77.

29. Chachay VS, Macdonald GA, Martin JH, Whitehead JP, O'Moore-Sullivan TM, Lee P, Franklin M, Klein K, Taylor PJ, Ferguson M, et al. Resveratrol Does Not Benefit Patients With Nonalcoholic Fatty Liver Disease. Clin Gastroenterol Hepatol 2014.

30. Janssens PL, Hursel R, Westerterp-Plantenga MS. Long-term green tea extract supplementation does not affect fat absorption, resting energy expenditure, and body composition in adults. J Nutr 2015;145(5):864-70.

31. Friedrich M, Petzke KJ, RaederstorffD, Wolfram S, Klaus S. Acute effects of epigallocatechin gallate from green tea on oxidation and tissue incorporation of dietary lipids in mice fed a high-fat diet. Int J Obes (Lond) 2012;36(5):735-43.

32. Jimenez-Gomez Y, Mattison JA, Pearson KJ, Martin-Montalvo A, Palacios HH, Sossong AM, Ward TM, Younts CM, Lewis K, Allard JS, et al. Resveratrol improves adipose insulin signaling and reduces the inflammatory response in adipose tissue of rhesus monkeys on high-fat, high-sugar diet. Cell Metab 2013;18(4):533-45.

33. Tchernof A, Despres JP. Pathophysiology of human visceral obesity: an update. Physiol Rev 2013;93(1):359-404.

34. Park SJ, Ahmad F, Philp A, Baar K, Williams T, Luo H, Ke H, Rehmann H, Taussig R, Brown AL, et al. Resveratrol ameliorates aging-related metabolic phenotypes by inhibiting cAMP phosphodiesterases. Cell 2012;148(3):421-33.

35. Bouskila M, Hunter RW, Ibrahim AF, Delattre L, Peggie M, van Diepen JA, Voshol PJ, Jensen J, Sakamoto K. Allosteric regulation of glycogen synthase controls glycogen synthesis in muscle. Cell Metab 2010;12(5):456-66.

36. Hoehn KL, Turner N, Swarbrick MM, Wilks D, Preston E, Phua Y, Joshi H, Furler SM, Larance M, Hegarty BD, et al. Acute or chronic upregulation of mitochondrial fatty acid oxidation has no net effect on whole-body energy expenditure or adiposity. Cell Metab 2010;11(1):70-6.

37. van de Weijer T, Sparks LM, Phielix E, Meex RC, van Herpen NA, Hesselink MK, Schrauwen P, Schrauwen-Hinderling VB. Relationships between mitochondrial function and metabolic flexibility in type 2 diabetes mellitus. PLoS One 2013;8(2):e51648.

38. Phielix E, Meex R, Ouwens DM, Sparks L, Hoeks J, Schaart G, Moonen-Kornips E, Hesselink MK, Schrauwen P. High oxidative capacity due to chronic exercise training attenuates lipid-induced insulin resistance. Diabetes 2012;61(10):2472-8. 
39. Corpeleijn E, Mensink M, Kooi ME, Roekaerts PM, Saris WH, Blaak EE. Impaired skeletal muscle substrate oxidation in glucose-intolerant men improves after weight loss. Obesity (Silver Spring) 2008;16(5):1025-32.

40. Hursel R, Janssens PL, Bouwman FG, Mariman EC, Westerterp-Plantenga MS. The Role of Catechol-O-Methyl Transferase Val(108/158)Met Polymorphism (rs4680) in the Effect of Green Tea on Resting Energy Expenditure and Fat Oxidation: A Pilot Study. PLoS One 2014;9(9):e106220.

41. Zhang K, Kaufman RJ. From endoplasmic-reticulum stress to the inflammatory response. Nature 2008;454(7203):455-62.

42. Jackson JR, Ryan MJ, Alway SE. Long-term supplementation with resveratrol alleviates oxidative stress but does not attenuate sarcopenia in aged mice. J Gerontol A Biol Sci Med Sci 2011;66(7):75164.

43. Serrano JC, Gonzalo-Benito H, Jove M, Fourcade S, Cassanye A, Boada J, Delgado MA, Espinel AE, Pamplona R, Portero-Otin M. Dietary intake of green tea polyphenols regulates insulin sensitivity with an increase in AMP-activated protein kinase alpha content and changes in mitochondrial respiratory complexes. Mol Nutr Food Res 2013;57(3):459-70.

44. Boudina S, Sena S, Sloan C, Tebbi A, Han YH, O'Neill BT, Cooksey RC, Jones D, Holland WL, McClain DA, et al. Early mitochondrial adaptations in skeletal muscle to diet-induced obesity are strain dependent and determine oxidative stress and energy expenditure but not insulin sensitivity. Endocrinology 2012;153(6):2677-88.

45. Liesa M, Shirihai OS. Mitochondrial dynamics in the regulation of nutrient utilization and energy expenditure. Cell Metab 2013;17(4):491-506.

46. Ukropcova B, McNeil M, Sereda O, de Jonge L, Xie H, Bray GA, Smith SR. Dynamic changes in fat oxidation in human primary myocytes mirror metabolic characteristics of the donor. J Clin Invest 2005;115(7):1934-41.

47. Cho SJ, Jung UJ, Choi MS. Differential effects of low-dose resveratrol on adiposity and hepatic steatosis in diet-induced obese mice. Br J Nutr 2012;108(12):2166-75.

48. Zhou J, Farah BL, Sinha RA, Wu Y, Singh BK, Bay BH, Yang CS, Yen PM. Epigallocatechin-3gallate (EGCG), a green tea polyphenol, stimulates hepatic autophagy and lipid clearance. PLoS One 2014;9(1):e87161.

49. Murase T, Nagasawa A, Suzuki J, Hase T, Tokimitsu I. Beneficial effects of tea catechins on dietinduced obesity: stimulation of lipid catabolism in the liver. Int J Obes Relat Metab Disord 2002;26(11):1459-64.

50. Calamini B, Ratia K, Malkowski MG, Cuendet M, Pezzuto JM, Santarsiero BD, Mesecar AD. Pleiotropic mechanisms facilitated by resveratrol and its metabolites. Biochem J 2010;429(2):27382 .

51. Lambert JD, Sang S, Yang CS. Biotransformation of green tea polyphenols and the biological activities of those metabolites. Mol Pharm 2007;4(6):819-25.

52. Overmyer KA, Evans CR, Qi NR, Minogue CE, Carson JJ, Chermside-Scabbo CJ, Koch LG, Britton SL, Pagliarini DJ, Coon JJ, et al. Maximal oxidative capacity during exercise is associated with skeletal muscle fuel selection and dynamic changes in mitochondrial protein acetylation. Cell Metab 2015;21(3):468-78.

53. Flatt JP, Ravussin E, Acheson KJ, Jequier E. Effects of dietary fat on postprandial substrate oxidation and on carbohydrate and fat balances. J Clin Invest 1985;76(3):1019-24. 
Supplemental Material 


\section{Supplemental Methods}

\section{Dual energy Xray absorptiometry}

Dual energy Xray absorptiometry measurements have been evaluated against computed tomography [1].

\section{Microarray analysis}

For microarray analysis, total RNA was extracted from skeletal muscle biopsies using the Trizol method (Qiagen, Venlo, Netherlands). $100 \mathrm{ng}$ of intact total RNA was processed applying the GeneChip $^{\circledR}$ WT PLUS Reagent Kit (Affymetrix, Santa Clara, CA, USA) according to the manufacture's manual. Fragmented and labelled single strand (ss)-cDNA (quantity and quality verified via Nanodrop- 8000 and Agilent 2100 Bioanalyzer) of each sample was hybridized onto a Human Transcriptome Array (HTA) 2.0 GeneChip ${ }^{\circledR}$ (Affymetrix). Hybridization, washing and scanning of the GeneChips was performed using the Affymetrix GeneChip ${ }^{\circledR}$ Hybridization oven 640, Fluidics Station 450/250 and Scanner 3000, according to the manufacture's user guides. Quality control of the scanned HTA 2.0 GeneChips was performed using the Expression Console application (Affymetrix Launcher software). Gene chip data were analyzed using the MADMAX database and analysis pipeline [2]. Probe sets were defined according to Dail et al. [3]. Ranked gene lists based on the intensity based moderated t-statistics [4] were used as input for Gene Set Enrichment Analysis, which was run with 1000 permutations [5, 6]. Functional data analysis was based upon FDR q-value $<0.2$ on the filtered data set (IQR $>0.2(\log 2)$, intensity $>20,>5$ arrays, $>5$ probes/gene). The data discussed in this publication have been deposited in NCBI's Gene Expression Omnibus [7] and are accessible through GEO Series accession number GSE71614 (http://www.ncbi.nlm.nih.gov/geo/query/acc.cgi?acc=GSE71614).

\section{Skeletal muscle lipid composition}

For determination of lipid composition, skeletal muscle biopsies were dissected free from extramyocellular adipose, blood and connective tissue before lyophilisation. Total lipids were extracted after addition of internal standards by the chloroform-methanol (2:1 vol/vol) method. After evaporation under nitrogen at $37^{\circ} \mathrm{C}$, lipids were separated into diacylglycerol and triacylglycerol by thin-layer chromatography and transferred into tubes for methylation with $1 \mathrm{~mL}$ of toluene-methanol BF3-methanol (14\%, 20-55-25\%). Incubation in capped tubes for 30 minutes at $100^{\circ} \mathrm{C}$ was followed by addition of $2 \mathrm{~mL}$ pentane to the samples and after vortexing and centrifugation $\left(1000 \mathrm{~g}, 5 \mathrm{~min}, 20^{\circ} \mathrm{C}\right)$ pentane extracts were isolated and evaporated (nitrogen, $30^{\circ} \mathrm{C}$ ) before fatty acid profiles were determined on an analytical gas chromatograph, as described previously [8].

\section{High-fat mixed meal composition}

Ingredients: $125 \mathrm{~g}$ whole milk (3.5\% fat), $15 \mathrm{~g}$ sugar, $50 \mathrm{~g}$ whipped crème, $150 \mathrm{~g}$ whipped crème ice 


\section{Macronutrient composition}

$\begin{array}{ll}\text { Energy content } & 625 \mathrm{kcal} \\ \text { Protein } & 6.3 \text { energy } \% \\ \text { Carbohydrate } & 32.6 \text { energy } \% \\ \text { Fat } & 61.2 \text { energy } \% \\ \text { Saturated fatty acids (FA) } & 35.5 \text { energy\% } \\ \text { Mono-unsaturated FA } & 18.8 \text { energy } \% \\ \text { Poly-unsaturated FA } & 1.7 \text { energy } \%\end{array}$

\section{Analysis of plasma epigallocatechin-3-gallate (EGCG) and resveratrol (RES) concentrations}

\section{Chemicals and reagents}

Trans-resveratrol (RES) and (-)-epigallocatechin-3-gallate (EGCG) were synthesized by DSM Nutritional Products Ltd. Dihydro-RES was purchased from abcam Biochemicals (Cambridge, UK) and $\beta$-glucuronidase was purchased from Sigma Aldrich (Buchs, Switzerland). Internal standard RES-phenyl- ${ }^{13} \mathrm{C}_{6}$ was purchased from Isoscience (King of Prussia, PA, US). Other chemicals used were analytical grade and obtained from Sigma-Aldrich (Buchs, Switzerland). Water used was passed through a Milli-Q water purification system (Merck Millipore, Darmstadt Germany).

\section{$\underline{\text { Resveratrol (RES) determination }}$}

\section{Instrumentation}

Separation and quantification was performed on an Agilent system coupling a 1290 HPLC with an AB Sciex QTRAP 4000 quadrupole mass spectrophotometer operating in negative TurboIonSpray mode. The analytical column was an Agilent Eclipse Plus Rapid Resolution HD C18 (2.1x50 mm, $1.8 \mu \mathrm{m})$. Samples were eluted using a binary reverse phase gradient (Chanel A: $5 \mathrm{mM}$ ammonium acetate and Chanel B: methanol) as follows: $0.00-0.50 \mathrm{~min}, 10 \% \mathrm{~B}$, flow rate $=0.6 \mathrm{~mL} / \mathrm{min} ; 0.50-2.50 \mathrm{~min}$ : gradient to $82 \% \mathrm{~B}$, flow rate $=0.6 \mathrm{~mL} / \mathrm{min} ; 2.50-2.60 \mathrm{~min}$ : gradient to $100 \% \mathrm{~B}$, flow rate $=0.8 \mathrm{~mL} / \mathrm{min} ; 2.60-4.00 \mathrm{~min}: 100 \% \mathrm{~B}$, flow rate $=800 \mu \mathrm{L} / \mathrm{min} ; 4.00$ $4.50 \mathrm{~min}$ : gradient to $10 \% \mathrm{~B}$, flow rate $=0.6 \mathrm{~mL} / \mathrm{min} ; 4.50-5.00 \mathrm{~min}: 10 \% \mathrm{~B}$, flow rate $=0.6 \mathrm{~mL} / \mathrm{min}$. Quantification was done by applying external calibration using internal standard (RES-phenyl- ${ }^{13} \mathrm{C}_{6}$ ). The criteria for identification and quantification were the retention time and MRM transition m/z 227/185 for trans-RES, m/z 229/123 for dihydro-RES and m/z 233/189 for RES-phenyl- ${ }^{13} \mathrm{C}_{6}$.

\section{Plasma sample preparation}

Immediately after centrifugation of the Vacutainer ${ }^{\circledR}$ tube containing EDTA, plasma samples were distributed in two tubes (with a minimum of $500 \mu \mathrm{L}$ plasma / tube) snap-frozen and stored at $80^{\circ} \mathrm{C}$ till analysis. For analysis $50 \mu \mathrm{L}$ of plasma sample, quality control or calibration samples were pipetted in Eppendorf tubes. $50 \mu \mathrm{L}$ of ammonium acetate buffer $200 \mathrm{mM}$ (for free form determination), or $50 \mu \mathrm{L}$ of $\beta$-glucuronidase solution at $5000 \mathrm{UI} / \mathrm{mL}$ (for total form determination), and $10 \mu \mathrm{L}$ of internal standard solution were added and mixed on a shaking system. For total form determination, the mixture was then incubated under shaking $(800 \mathrm{rpm})$ at $37^{\circ} \mathrm{C}$ for $5 \mathrm{~h} \mathrm{before} \mathrm{the}$ extraction steps. Then $400 \mu \mathrm{L}$ of ethyl acetate was added to the samples and mixed. Afterwards, 
the samples were centrifuged for $5 \mathrm{~min}$ at $3000 \mathrm{rpm}$ at approximately $8^{\circ} \mathrm{C} .250 \mu \mathrm{L}$ supernatant was then evaporated to dryness under nitrogen at nominal $40^{\circ} \mathrm{C}$. Finally, the residue was dissolved in $200 \mu \mathrm{L}$ of injection solvent (methanol/ammonium acetate $5 \mathrm{mM}(90: 10, \mathrm{v} / \mathrm{v})$ ) and placed in the auto-sampler for injection.

\section{(-)-Epigallocatechin-3-gallate (EGCG) determination}

\section{Instrumentation}

Separation and quantification was performed on an Agilent system coupling a 1290 HPLC with an AB Sciex QTRAP 4000 quadrupole mass spectrophotometer operating in negative TurboIonSpray mode. The analytical column was an Agilent Eclipse Plus Rapid Resolution HD C18 (2.1x50 mm, $1.8 \mu \mathrm{m})$. Samples were eluted using a binary reverse phase gradient (Chanel A: $0.1 \%$ formic acid and Chanel B: acetonitrile $+0.1 \%$ formic acid) as follows: $0.00-0.50 \mathrm{~min}, 10 \%$ $\mathrm{B}$, flow rate $=0.6 \mathrm{~mL} / \mathrm{min}$; $0.50-2.50 \mathrm{~min}$ : gradient to $82 \% \mathrm{~B}$, flow rate $=0.6 \mathrm{~mL} / \mathrm{min} ; 2.50-2.60$ min: gradient to $100 \% \mathrm{~B}$, flow rate $=0.8 \mathrm{~mL} / \mathrm{min} ; 2.60-4.00 \mathrm{~min}: 100 \% \mathrm{~B}$, flow rate $=800 \mu \mathrm{L} / \mathrm{min}$; 4.00-4.50 min: gradient to $10 \% \mathrm{~B}$, flow rate $=0.6 \mathrm{~mL} / \mathrm{min}$; $4.50-5.00 \mathrm{~min}: 10 \% \mathrm{~B}$, flow rate $=0.6$ $\mathrm{mL} / \mathrm{min}$. Quantification was done by applying external calibration using internal standard ((-)gallocatechin gallate (GCG)). The criteria for identification and quantification were the retention time and MRM transition m/z 457/169 for EGCG and for internal standard.

\section{Plasma sample preparation}

Immediately after centrifugation of the Vacutainer ${ }^{\circledR}$ tube containing K-EDTA, $500 \mu \mathrm{L}$ plasma were collected in a tube and mixed at room temperature with $500 \mu \mathrm{L}$ stabilizing buffer $[100 \mathrm{mg}$ ethylenediaminetetraacetic acid (EDTA) disodium salt dihydrate and $10 \mathrm{~g}$ ascorbic acid were dissolved in $1000 \mathrm{~mL}$ sodium acetate buffer (sodium acetate buffer $35 \mathrm{mM}: 2.38 \mathrm{~g}$ sodium acetate trihydrate and $1.00 \mathrm{~mL}$ acetic acid dissolved in water and adjusted to $1000 \mathrm{~mL}$ ), pH 5.0], snapfrozen in liquid nitrogen and stored at $-80^{\circ} \mathrm{C}$ till analysis.

To determine free EGCG, $50 \mu \mathrm{L}$ internal standard (GCG) was added to $200 \mu \mathrm{L}$ stabilized plasma followed by a two times liquid-liquid extraction with ethyl acetate. After centrifugation, an aliquot of the organic phase is evaporated to dryness, re-dissolved in $0.1 \%$ formic acid and placed in the auto-sampler for injection.

\section{Validation}

The analytical methods used for the quantification of polyphenols in plasma samples were validated according to the following guidelines: Guideline on Bioanalytical Method Validation (9) and Guidance for industry: Bioanalytical Method Validation (10). To assess the daily and longterm laboratory performance of the method, dedicated standard and quality control samples have been analyzed daily with the unknown samples to ensure the accuracy and precision of the methods ( $\pm 15 \% ; \pm 20 \%$ for lower limit of quantification (LLQ)-value). For EGCG, RES and dihydro-RES the detection limits were $2.5,10$ and $10 \mathrm{ng} / \mathrm{mL}(5.45,43.8$ and $43.4 \mathrm{nM})$, respectively. 


\section{Supplemental Results}

Supplemental Table S5.1: Condensed selection of significantly affected pathways by EGCG+RES and PLA supplementation in skeletal muscle identified with GSEA

\begin{tabular}{|c|c|c|}
\hline Gene set & NES & $\begin{array}{l}\text { FDR } \\
\text { q- } \\
\text { value }\end{array}$ \\
\hline \multicolumn{3}{|l|}{ Placebo - up-regulated (limited to NES $\geq 2.1$ ) } \\
\hline GENE.EXPRESSION & 2.45 & 0.00 \\
\hline WP2005.MIR.TARGETED.GENES.IN.MUSCLE.CELL.TARBASE & 2.43 & 0.00 \\
\hline L13A.MEDIATED.TRANSLATIONAL.SILENCING.OF.CERULOPLASMIN.EXPRESSION & 2.43 & 0.00 \\
\hline X3.UTR.MEDIATED.TRANSLATIONAL.REGULATION & 2.43 & 0.00 \\
\hline GTP.HYDROLYSIS.AND.JOINING.OF.THE.60S.RIBOSOMAL.SUBUNIT & 2.40 & 0.00 \\
\hline EUKARYOTIC.TRANSLATION.INITIATION & 2.40 & 0.00 \\
\hline TRANSLATION & 2.39 & 0.00 \\
\hline MRNA.SPLICING.MAJOR.PATHWAY & 2.39 & 0.00 \\
\hline PROCESSING.OF.CAPPED.INTRON.CONTAINING.PRE.MRNA & 2.39 & 0.00 \\
\hline MRNA.SPLICING & 2.38 & 0.00 \\
\hline FORMATION.OF.A.POOL.OF.FREE.4OS.SUBUNITS & 2.36 & 0.00 \\
\hline CAP.DEPENDENT.TRANSLATION.INITIATION & 2.35 & 0.00 \\
\hline METABOLISM.OF.MRNA & 2.35 & 0.00 \\
\hline HIV.INFECTION & 2.34 & 0.00 \\
\hline KEGG_RIBOSOME & 2.34 & 0.00 \\
\hline METABOLISM.OF.RNA & 2.34 & 0.00 \\
\hline KEGG_SPLICEOSOME & 2.33 & 0.00 \\
\hline MITOTIC.ANAPHASE & 2.32 & 0.00 \\
\hline WP2684.HOST.INTERACTIONS.OF.HIV.FACTORS & 2.32 & 0.00 \\
\hline NONSENSE.MEDIATED.DECAY.NMD.ENHANCED.BY.THE.EXON.JUNCTION.COMPLEX.EJC. & 2.31 & 0.00 \\
\hline WP1864.MUSCLE.CONTRACTION & 2.31 & 0.00 \\
\hline NONSENSE.MEDIATED.DECAY.NMD. & 2.31 & 0.00 \\
\hline SEPARATION.OF.SISTER.CHROMATIDS & 2.30 & 0.00 \\
\hline WP2757.MITOTIC.METAPHASE.AND.ANAPHASE & 2.30 & 0.00 \\
\hline SRP.DEPENDENT.COTRANSLATIONAL.PROTEIN.TARGETING.TO.MEMBRANE & 2.29 & 0.00 \\
\hline MITOTIC.METAPHASE.AND.ANAPHASE & 2.29 & 0.00 \\
\hline WP2004.MIR.TARGETED.GENES.IN.LYMPHOCYTES.TARBASE & 2.28 & 0.00 \\
\hline TRANSLOCATION.OF.GLUT4.TO.THE.PLASMA.MEMBRANE & 2.28 & 0.00 \\
\hline HOST.INTERACTIONS.OF.HIV.FACTORS & 2.26 & 0.00 \\
\hline AUTODEGRADATION.OF.CDH1.BY.CDH1.APC.C & 2.26 & 0.00 \\
\hline WP411.MRNA.PROCESSING & 2.26 & 0.00 \\
\hline RNA.POLYMERASE.II.TRANSCRIPTION & 2.26 & 0.00 \\
\hline KEGG_RNA.TRANSPORT & 2.26 & 0.00 \\
\hline INFLUENZZA.INFECTION & 2.26 & 0.00 \\
\hline INFLUENZA.LIFE.CYCLE & 2.25 & 0.00 \\
\hline PEPTIDE.CHAIN.ELONGATION & 2.25 & 0.00 \\
\hline WP2746.SIGNALING.BY.THE.B.CELL.RECEPTOR.BCR. & 2.25 & 0.00 \\
\hline CELL.CYCLE & 2.24 & 0.00 \\
\hline EUKARYOTIC.TRANSLATION.TERMINATION & 2.24 & 0.00 \\
\hline $\begin{array}{l}\text { NONSENSE.MEDIATED.DECAY.NMD.INDEPENDENT.OF.THE.EXON.JUNCTION.COMPLEX.EJC. } \\
\text { PHOSPHORYLATION.SITE.MUTANTS.OF.CTNNB1.ARE.NOT.TARGETED.TO.THE.PROTEASOM }\end{array}$ & 2.23 & 0.00 \\
\hline E.BY.THE.DESTRUCTION.COMPLEX & 2.22 & 0.00 \\
\hline WP2777.TRANSLOCATION.OF.GLUT4.TO.THE.PLASMA.MEMBRANE & 2.21 & 0.00 \\
\hline WP2002.MIR.TARGETED.GENES.IN.EPITHELIUM.TARBASE & 2.21 & 0.00 \\
\hline MUSCLE.CONTRACTION & 2.21 & 0.00 \\
\hline
\end{tabular}


ACTIVATION.OF.NF.KAPPAB.IN.B.CELLS

DELETIONS.IN.THE.AXIN.GENES.IN.HEPATOCELLULAR.CARCINOMA.RESULT.IN.ELEVATED. WNT.SIGNALING

APC.C.CDH1.MEDIATED.DEGRADATION.OF.CDC20.AND.OTHER.APC.C.CDH1.TARGETED.PR OTEINS.IN.LATE.MITOSIS.EARLY.G1

M.PHASE

INFLUENZA.VIRAL.RNA.TRANSCRIPTION.AND.REPLICATION

VIRAL.MRNA.TRANSLATION

APC.C.MEDIATED.DEGRADATION.OF.CELL.CYCLE.PROTEINS

REGULATION.OF.MITOTIC.CELL.CYCLE

MISSPLICED.GSK3BETA.MUTANTS.STABILIZE.BETA.CATENIN

ANTIGEN.PROCESSING.UBIQUITINATION.PROTEASOME.DEGRADATION

AXIN.MUTANTS.DESTABILIZE.THE.DESTRUCTION.COMPLEX.ACTIVATING.WNT.SIGNALING EUKARYOTIC.TRANSLATION.ELONGATION

WP1889.PROCESSING.OF.CAPPED.INTRON.CONTAINING.PRE.MRNA

APC.TRUNCATION.MUTANTS.HAVE.IMPAIRED.AXIN.BINDING

DEGRADATION.OF.BETA.CATENIN.BY.THE.DESTRUCTION.COMPLEX

TCF7L2.MUTANTS.DON.T.BIND.CTBP

HIV.LIFE.CYCLE

WP2683.INFLUENZA.LIFE.CYCLE

S45.MUTANTS.OF.BETA.CATENIN.AREN.T.PHOSPHORYLATED

DELETIONS.IN.THE.AMER1.GENE.DESTABILIZE.THE.DESTRUCTION.COMPLEX

TRUNCATIONS.OF.AMER1.DESTABILIZE.THE.DESTRUCTION.COMPLEX

CYCLIN.A.CDK2.ASSOCIATED.EVENTS.AT.S.PHASE.ENTRY

APC.TRUNCATION.MUTANTS.ARE.NOT.K63.POLYUBIQUITINATED

T41.MUTANTS.OF.BETA.CATENIN.AREN.T.PHOSPHORYLATED

VIF.MEDIATED.DEGRADATION.OF.APOBEC3G

S33.MUTANTS.OF.BETA.CATENIN.AREN.T.PHOSPHORYLATED

AMER1.MUTANTS.DESTABILIZE.THE.DESTRUCTION.COMPLEX

CELL.CYCLE.MITOTIC

DEGRADATION.OF.GLI1.BY.THE.PROTEASOME

S37.MUTANTS.OF.BETA.CATENIN.AREN.T.PHOSPHORYLATED

LATE.PHASE.OF.HIV.LIFE.CYCLE

REGULATION.OF.APC.C.ACTIVATORS.BETWEEN.G1.S.AND.EARLY.ANAPHASE

AXIN.MISSENSE.MUTANTS.DESTABILIZE.THE.DESTRUCTION.COMPLEX

TRANSLATION.INITIATION.COMPLEX.FORMATION

ACTIVATION.OF.THE.MRNA.UPON.BINDING.OF.THE.CAP.BINDING.COMPLEX.AND.EIFS.AND.S UBSEQUENT.BINDING.TO.43S

CYCLIN.E.ASSOCIATED.EVENTS.DURING.G1.S.TRANSITION

REGULATION.OF.APOPTOSIS

WP1782.APC.C.MEDIATED.DEGRADATION.OF.CELL.CYCLE.PROTEINS

TRUNCATED.APC.MUTANTS.DESTABILIZE.THE.DESTRUCTION.COMPLEX

APC.C.CDC20.MEDIATED.DEGRADATION.OF.MITOTIC.PROTEINS

FORMATION.OF.THE.TERNARY.COMPLEX.AND.SUBSEQUENTLY.THE.43S.COMPLEX

UBIQUITIN.DEPENDENT.DEGRADATION.OF.CYCLIN.D

CDC20.PHOSPHO.APC.C.MEDIATED.DEGRADATION.OF.CYCLIN.A

APC.C.CDC20.MEDIATED.DEGRADATION.OF.SECURIN

REGULATION.OF.ORNITHINE.DECARBOXYLASE.ODC.

KEGG_MRNA.SURVEILLANCE.PATHWAY

SCF.BETA.TRCP.MEDIATED.DEGRADATION.OF.EMI1

VPU.MEDIATED.DEGRADATION.OF.CD4

WP1848.METABOLISM.OF.CARBOHYDRATES

ACTIVATION.OF.APC.C.AND.APC.C.CDC20.MEDIATED.DEGRADATION.OF.MITOTIC.PROTEINS

KEGG_PROTEIN.PROCESSING.IN.ENDOPLASMIC.RETICULUM
2.21 


\begin{tabular}{lcc} 
RIBOSOMAL.SCANNING.AND.START.CODON.RECOGNITION & & \\
CLASS.I.MHC.MEDIATED.ANTIGEN.PROCESSING.PRESENTATION & 2.10 & 0.00 \\
\hline Placebo - down-regulated & 2.10 & 0.00 \\
\hline GPCR.LIGAND.BINDING & & \\
WP1825.GPCR.LIGAND.BINDING & -2.03 & 0.08 \\
CLASS.A.1.RHODOPSIN.LIKE.RECEPTORS. & -2.05 & 0.09 \\
WP455.GPCRS.CLASS.A.RHODOPSIN.LIKE & -1.97 & 0.09 \\
KEGG_NEUROACTIVE.LIGAND.RECEPTOR.INTERACTION & -1.99 & 0.09 \\
\hline EGCG+RES - UP-regulated (limited to NES $\geq 1.8)$ & -2.08 & 0.13 \\
\hline KEGG_VALINE.LEUCINE.AND.ISOLEUCINE.DEGRADATION & & \\
PYRUVATE.METABOLISM.AND.CITRIC.ACID.TCA.CYCLE & 1.83 & 0.10 \\
WP2677.SIGNALING.BY.TYPE.1.INSULIN.LIKE.GROWTH.FACTOR.1.RECEPTOR.IGF1R. & 1.85 & 0.10 \\
WP2023.CELL.DIFFERENTIATION.META & 1.84 & 0.10 \\
WP1935.TRANSPORT.OF.GLUCOSE.AND.OTHER.SUGARS.BILE.SALTS.AND.ORGANIC.ACIDSS. & 1.83 & 0.10 \\
METAL.IONS.AND.AMINE.COMPOUNDS & 1.80 & 0.11 \\
KEGG_DILATED.CARDIOMYOPATHY & 1.85 & 0.11 \\
WP2766.THE.CITRIC.ACID.TCA.CYCLE.AND.RESPIRATORY.ELECTRON.TRANSPORT. & 1.94 & 0.11 \\
KEGG_CALCIUM.SIGNALING.PATHWAY & 1.81 & 0.11 \\
TRANSCRIPTION.COUPLED.NER.TC.NER. & 1.81 & 0.11 \\
CITRIC.ACID.CYCLE.TCA.CYCLE. & 1.87 & 0.12 \\
WP1848.METABOLISM.OF.CARBOHYDRATES & 1.85 & 0.12 \\
WP78.TCA.CYCLE & 1.87 & 0.13 \\
KEGG_CITRATE.CYCLE.TCA.CYCLE. & 1.88 & 0.14 \\
WP534.GLYCOLYSIS.AND.GLUCONEOGENESIS & 1.95 & 0.15 \\
PI3K.CASCADE & 1.89 & 0.16 \\
\hline EGCG+RES - down-regulated & & \\
\hline PHASE.1.FUNCTIONALIZATION.OF.COMPOUNDS & -2.05 & 0.04 \\
WP43.OXIDATION.BY.CYTOCHROME.P450 & -1.76 & 0.19 \\
KEGG_METABOLISM.OF.XENOBIOTICS.BY.CYTOCHROME.P450 & -1.79 & 0.19 \\
CYTOCHROME.P450.ARRANGED.BY.SUBSTRATE.TYPE & -1.82 & 0.19 \\
\hline
\end{tabular}

EGCG+RES, epigallocatechin-3-gallate plus resveratrol; GESA, gene set enrichment analysis; NES, normalized enrichment score; $q$ indicates statistical significance of false discovery rate (FDR) of post versus pre-enrichment 


\section{Supplemental Table S5.2: Skeletal muscle lipid fractions before and after 12 wk of supplementation with EGCG+RES or PLA}

\begin{tabular}{|c|c|c|c|c|c|c|c|}
\hline & \multicolumn{2}{|c|}{$\begin{array}{c}\text { PLA } \\
(n=17)\end{array}$} & \multicolumn{2}{|c|}{$\begin{array}{c}\text { EGCG+RES } \\
(n=12)\end{array}$} & \multirow{2}{*}{$\frac{\text { Time }}{\mathrm{P}}$} & \multirow{2}{*}{$\begin{array}{c}\text { Treatment } \\
\mathrm{P}\end{array}$} & \multirow{2}{*}{$\begin{array}{c}\text { Time*treatmen } \\
\text { P }\end{array}$} \\
\hline & Week 0 & Week 12 & Week 0 & Week 12 & & & \\
\hline \multicolumn{8}{|l|}{ DAG } \\
\hline Total, $\mu \mathrm{mol} / \mathrm{g}$ & $13.6 \pm 1.6$ & $11.7 \pm 1.9$ & $20.4 \pm 6.9$ & $14.9 \pm 2.8$ & 0.23 & 0.22 & 0.52 \\
\hline SFA, $\%$ & $35.4 \pm 2.0$ & $36.9 \pm 2.6$ & $37.4 \pm 2.6$ & $37.0 \pm 2.4$ & 0.68 & 0.72 & 0.59 \\
\hline MUFA, \% & $52.8 \pm 1.7$ & $51.0 \pm 2.4$ & $49.8 \pm 3.4$ & $49.7 \pm 3.5$ & 0.61 & 0.48 & 0.70 \\
\hline PUFA, \% & $11.8 \pm 0.5$ & $12.1 \pm 0.8$ & $12.8 \pm 1.2$ & $13.3 \pm 1.3$ & 0.62 & 0.29 & 0.92 \\
\hline \multicolumn{8}{|l|}{ TAG } \\
\hline Total, $\mu \mathrm{mol} / \mathrm{g}$ & $308 \pm 103$ & $285 \pm 98$ & $392 \pm 137$ & $154 \pm 31$ & 0.24 & 0.83 & 0.27 \\
\hline SFA, $\%$ & $38.9 \pm 2.1$ & $40.1 \pm 2.2$ & $43.9 \pm 3.9$ & $42.9 \pm 3.8$ & 0.88 & 0.27 & 0.62 \\
\hline MUFA, \% & $51.1 \pm 1.6$ & $50.0 \pm 1.8$ & $44.7 \pm 4.4$ & $46.8 \pm 4.0$ & 0.93 & 0.13 & 0.55 \\
\hline PUFA, \% & $10.1 \pm 0.6$ & $9.9 \pm 0.6$ & $11.4 \pm 1.3$ & $10.4 \pm 1.3$ & 0.37 & 0.42 & 0.52 \\
\hline
\end{tabular}

Values are means $\pm S E M s$. Data were analyzed by using a two-factor repeated-measures ANOVA, with time ( $P_{\text {time, }}$ pre, post) and treatment (Ptreatment, PLA, EGCG+RES) as factors. $P<0.05$ was considered significant. DAG, diacylglycerol; EGCG+RES, epigallocatechin-3-gallate plus resveratrol; MUFA, mono-unsaturated fatty acids; PUFA, poly-unsaturated fatty acids; PLA, placebo; post, after intervention; pre, before intervention; SFA, saturated fatty acids; TAG, triacylglycerol.

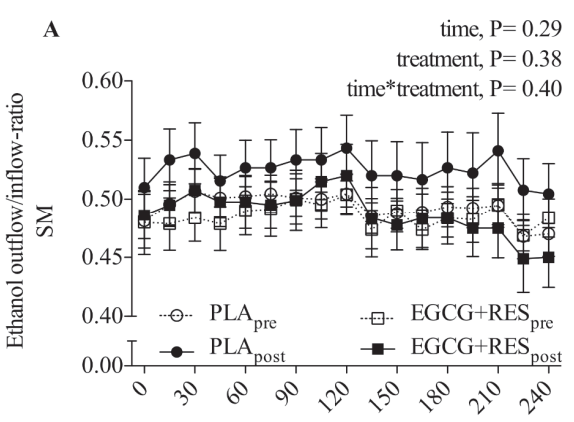

time, $\min$

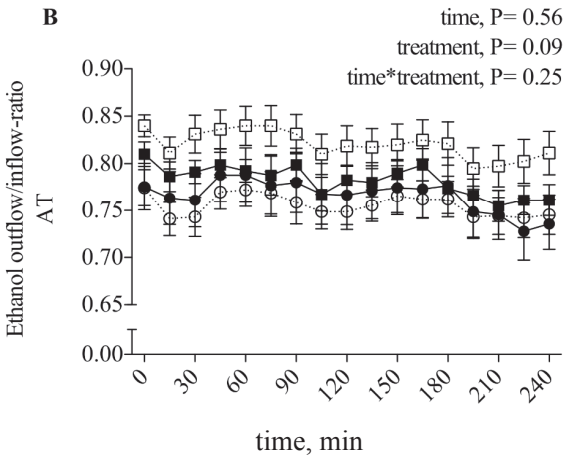

\section{Supplemental Figure S5.1: Local fasting and postprandial interstitial blood flow before and after the intervention}

Values are means \pm SEMs $(n=17)$. Interstitial local blood flows (ethanol outflow/inflow-ratios) were measured after an overnight fast (at $0 \mathrm{~min}$ ) and postprandial (from 0 to $240 \mathrm{~min}$ ) by means of microdialysis in skeletal muscle (M. gastrocnemius; A) and abdominal subcutaneous adipose tissue $(B)$ of male subjects. Fasting values ( $t=0$ min), AUCs during the HFMM test (calculated from $t=0-240 \mathrm{~min}$ ) and the incremental AUCs were analyzed using a two-factor repeated-measures ANOVA, with time ( $P_{\text {time, }}$ pre, post) and treatment ( $P_{\text {treatment, }}$ PLA, EGCG+RES) as factors. $P<0.05$ was considered significant. Reported $P$-values refer to the AUCs of the respective metabolite concentrations. AT, adipose tissue; EGCG+RES, epigallocatechin-3-gallate plus resveratrol; PLA, placebo; post, after intervention; pre, before intervention; SM, skeletal muscle. Dashed line, open circles: PLApre; solid line, filled circles: PLApost; Dashed line, open squares: EGCG+RESpre; solid line, filled squares: EGCG+RESpost. 

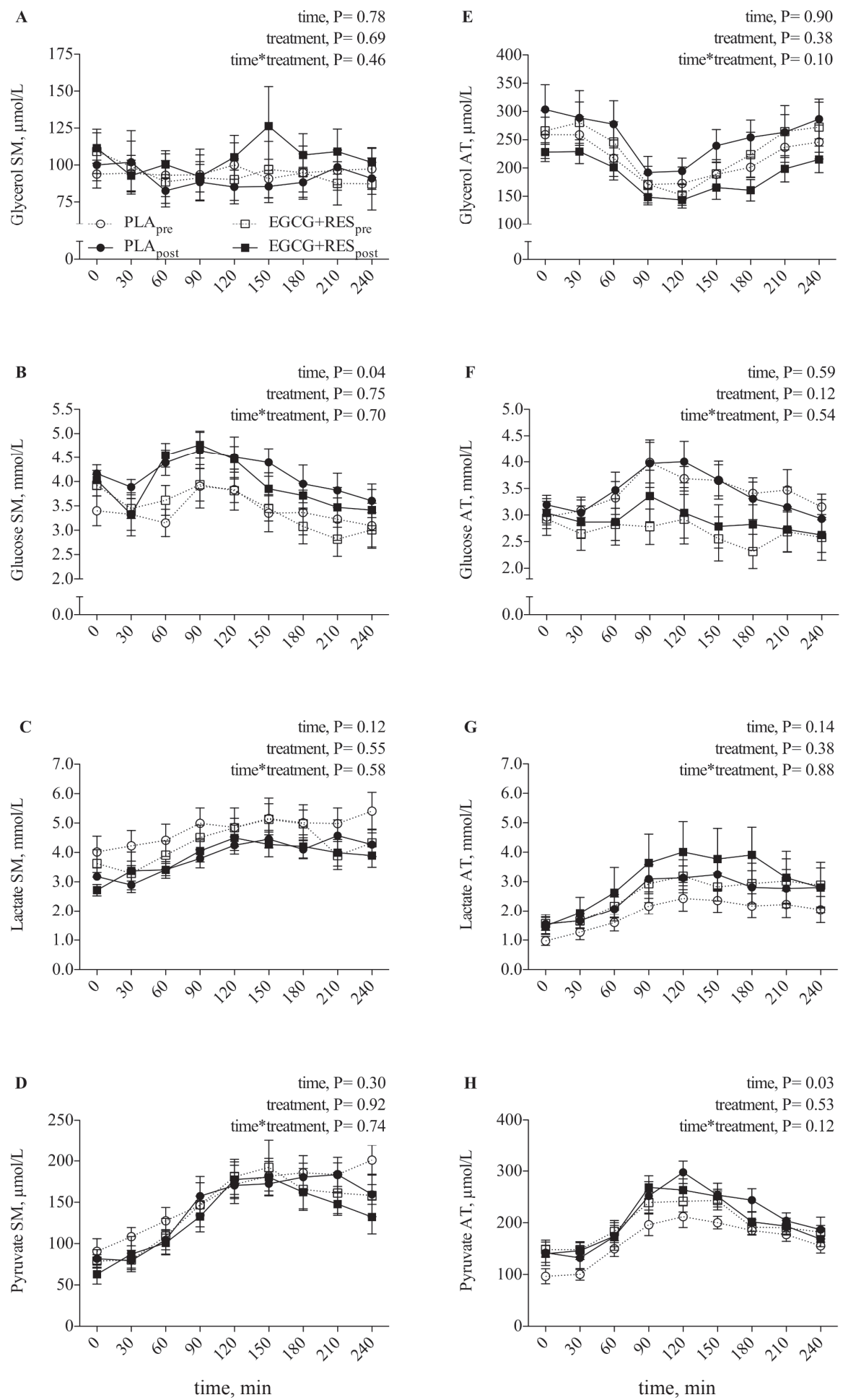


\section{Supplemental Figure S5.2: Local fasting and postprandial interstitial metabolite concentrations before and after the intervention}

Values are means \pm SEMs $(n=17)$. Interstitial metabolite concentrations (glycerol, glucose, lactate and pyruvate) were measured after an overnight fast (at $0 \mathrm{~min}$ ) and postprandial (from 0 to $240 \mathrm{~min}$ ) by means of microdialysis in skeletal muscle ( $M$. gastrocnemius; $A-D$ ) and abdominal subcutaneous adipose tissue $(E-H)$ of male subjects. Fasting values ( $t=0$ min), AUCs during the HFMM test (calculated from $\mathrm{t}=0-240 \mathrm{~min}$ ) and the incremental AUCs were analyzed using a two-factor repeated-measures ANOVA, with time ( $P_{\text {time, }}$ pre, post) and treatment ( $\left.P_{\text {treatment, }} P L A, E G C G+R E S\right)$ as factors. $P<0.05$ was considered significant. Reported P-values refer to the AUCs of the respective metabolite concentrations. AT, adipose tissue; EGCG+RES, epigallocatechin-3-gallate plus resveratrol; PLA, placebo; post, after intervention; pre, before intervention; SM, skeletal muscle. Dashed line, open circles: PLApre; solid line, filled circles: PLApost; Dashed line, open squares: EGCG+RESpre; solid line, filled squares: $E G C G+R E S$ post.

\section{Supplemental References}

1. Micklesfield LK, Goedecke JH, Punyanitya M, Wilson KE, Kelly TL. Dual-energy X-ray performs as well as clinical computed tomography for the measurement of visceral fat. Obesity (Silver Spring) 2012;20(5):1109-14. doi: 10.1038/oby.2011.367.

2. Lin K, Kools H, de Groot PJ, Gavai AK, Basnet RK, Cheng F, Wu J, Wang X, Lommen A, Hooiveld GJ, et al. MADMAX - Management and analysis database for multiple omics experiments. J Integr Bioinform 2011;8(2):160. doi: 10.2390/biecoll-jib-2011-160.

3. Dai MH, Wang PL, Boyd AD, Kostov G, Athey B, Jones EG, Bunney WE, Myers RM, Speed TP, Akil $\mathrm{H}$, et al. Evolving gene/transcript definitions significantly alter the interpretation of GeneChip data. Nucleic Acids Research 2005;33(20). doi: 10.1093/nar/gni179.

4. Sartor MA, Tomlinson CR, Wesselkamper SC, Sivaganesan S, Leikauf GD, Medvedovic M. Intensity-based hierarchical Bayes method improves testing for differentially expressed genes in microarray experiments. Bmc Bioinformatics 2006;7. doi: 10.1186/1471-2105-7-538.

5. Subramanian A, Tamayo P, Mootha VK, Mukherjee S, Ebert BL, Gillette MA, Paulovich A, Pomeroy SL, Golub TR, Lander ES, et al. Gene set enrichment analysis: A knowledge-based approach for interpreting genome-wide expression profiles. Proceedings of the National Academy of Sciences of the United States of America 2005;102(43):15545-50. doi: 10.1073/Pnas.0506580102.

6. Mootha VK, Lindgren CM, Eriksson KF, Subramanian A, Sihag S, Lehar J, Puigserver P, Carlsson E, Ridderstrale M, Laurila E, et al. PGC-1 alpha-responsive genes involved in oxidative phosphorylation are coordinately downregulated in human diabetes. Nature Genetics 2003;34(3):267-73. doi: 10.1038/Ng1180.

7. Edgar R, Domrachev M, Lash AE. Gene Expression Omnibus: NCBI gene expression and hybridization array data repository. Nucleic Acids Research 2002;30(1):207-10.

8. van Hees AM, Jans A, Hul GB, Roche HM, Saris WH, Blaak EE. Skeletal muscle fatty acid handling in insulin resistant men. Obesity (Silver Spring) 2011;19(7):1350-9.

9. European Medicines Agency. Guideline on Bioanalytical Method Validation. In: Use CfMPfH, ed. London, UK, 2011.

10. Food and Drug Administration US. Guidance for industry: Bioanalytical Method Validation. In: Medicine DoHaHS-CfDEaRaCfV, ed. Silver Spring, MD, US, 2001. 


\section{CHAPTER 6}

\section{The effects of polyphenol supplementation on adipose tissue morphology and gene expression in overweight and obese humans}

Jasper Most, Ines Warnke, Mark v. Boekschoten, Johan W.E. Jocken, Philip de Groot, Angelika Friedel, Igor Bendik, Gijs H. Goossens, Ellen E. Blaak

"Adipocyte", under revision 


\section{Abstract}

Background: Dietary polyphenols may have beneficial effects on adipose tissue mass and function in rodents, but human studies are scarce.

Methods: In a randomized, placebo-controlled study, 25 (10 women) overweight and obese humans received a combination of polyphenols epigallocatechin-gallate and resveratrol $(282 \mathrm{mg} / \mathrm{d}, 80 \mathrm{mg} / \mathrm{d}$, respectively, EGCG+RES, n=11) or placebo (PLA, n=14) supplementation for 12 weeks. Adipose tissue biopsies were collected for assessment of adipocyte morphology and microarray analysis.

Results: EGCG+RES had no effects on adipocyte size and distribution compared with PLA. However, we identified that pathways contributing to adipogenesis, cell cycle and apoptosis were significantly downregulated by EGCG+RES versus PLA. Furthermore, EGCG+RES significantly decreased expression of pathways related to energy metabolism, oxidative stress, inflammation, and immune defense as compared with PLA.

Conclusion: In conclusion, the adipose tissue gene expression profile indicates a reduced cell turnover after 12-week EGCG+RES supplementation in overweight-obese subjects. It remains to be elucidated whether these alterations translate into long term metabolic effects.

\section{Graphical Abstract}

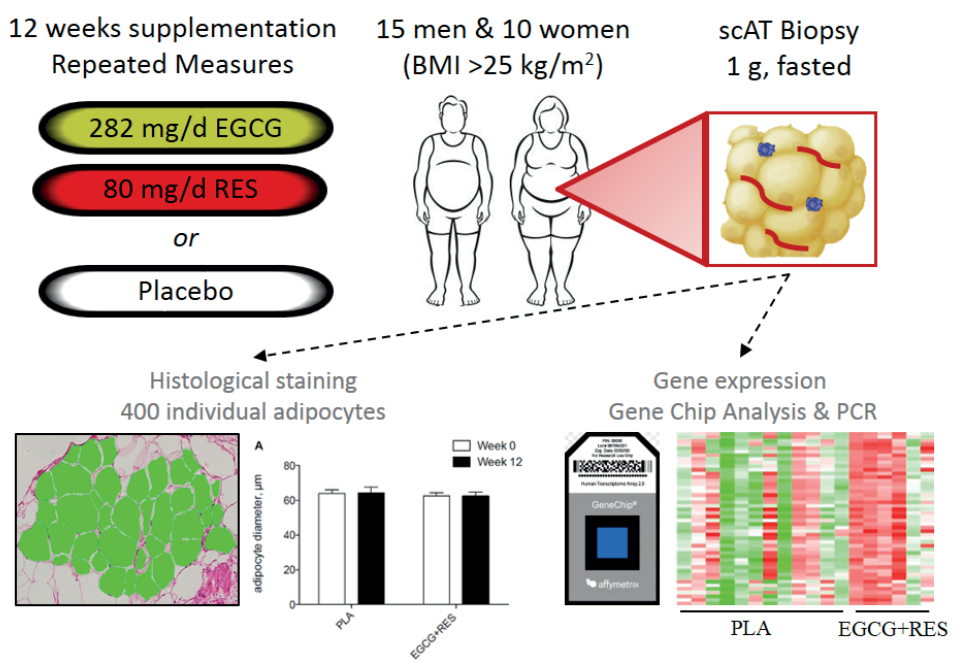

Polyphenols are naturally abundant ingredients in tea (EGCG) and wine (RES) and may improve metabolic health through effects on adipose tissue (AT). Overweight subjects consumed either polyphenol-supplements or placebos (PLA) for 12 weeks. We observed no effect on fat cell size, but the expression of genes related to inflammation and metabolism was lower after the polyphenol-intervention vs PLA. While a reduction in inflammatory pathways may be beneficial towards metabolic health, a reduction in fat cell metabolism may impair the physiological function of AT 


\section{Background}

Enlargement of abdominal subcutaneous adipocytes is an independent marker of insulin resistance [1], and predicts the development of type 2 diabetes [2]. Additionally, adipose tissue (AT) dysfunction rather than an increased fat mass per se is associated with the development of insulin resistance. This suggests that both the morphology and function of AT determine the risk of developing chronic metabolic conditions accompanying the obese phenotype.

Epigallocatechin-3-gallate (EGCG) and resveratrol (RES) are dietary polyphenols, abundantly available in green tea and in grapes, respectively. Both have been shown to prevent the development of fat mass accretion and insulin resistance in rodents on obesogenic diets via inhibition of adipogenesis and inflammation, and increased lipolysis and energy expenditure [36]. However, most human studies have not found significant effects on AT mass and whole-body metabolic profile after supplementation with either EGCG or RES [7, 8]. Nevertheless, RES supplementation for 4 weeks induced a reduction in adipocyte size in obese men [9], which was accompanied by an AT gene expression profile indicative of increased adipose tissue turnover including adipogenesis, autophagy and inflammation.

We have recently postulated that combining different polyphenols may lead to additional and/or synergistic and, therefore, more pronounced metabolic effects compared with single supplementation. Indeed, while EGCG supplementation alone for 3 days was ineffective [10], combined EGCG and RES (EGCG+RES) supplementation increased energy expenditure and plasma leptin concentrations in overweight subjects [11]. More recently, we have shown that combined EGCG+RES supplementation for 12 weeks increased whole-body fat oxidation and mitochondrial capacity in skeletal muscle, but did not significantly alter whole-body lipolysis, AT and skeletal muscle lipolysis and tissue-specific insulin sensitivity [12]. Here, we investigated whether EGCG+RES supplementation for 12 weeks induces alterations in abdominal subcutaneous AT morphology and gene expression profiles in overweight and obese men and women compared with placebo (PLA).

\section{Material and Methods}

\section{Study design}

The current study was conducted as part of a randomized, double-blind placebo-controlled intervention trial. The effects of 12 weeks EGCG+RES supplementation $(282 \mathrm{mg} / \mathrm{d}$ and $80 \mathrm{mg} / \mathrm{d}$, respectively) on insulin sensitivity and lipid metabolism were investigated in 38 overweight and 
obese men and premenopausal women [12]. These doses have been shown safe and effective before $[9,11]$. This trial was registered at clinicaltrials.gov (NCT02381145).

Before and after intervention, an abdominal subcutaneous AT biopsy was collected after an overnight fast. All subjects gave written informed consent for participation in the original study, which was approved by the Medical Ethical Committee of Maastricht University Medical Center+. All procedures were conducted according to the Declaration of Helsinki.

\section{Abdominal subcutaneous adipocyte size}

AT biopsies $(\sim 1 \mathrm{~g})$ were taken before and after intervention under local anesthesia using a needle biopsy technique [22], snap-frozen using liquid nitrogen and stored at $-80^{\circ} \mathrm{C}$ until further analyses. One portion of the biopsy was embedded in paraffin, of which sections were cut for staining (hematoxylin and eosin), digital imaging and computerized measurement of 400 individual adipocytes to determine adipocyte morphology [22].

\section{Adipose tissue gene and protein expression}

For gene expression analysis, total RNA was extracted from frozen AT aliquots ( $300 \mathrm{mg})$ using the Trizol method (Qiagen, Venlo, Netherlands). Starting from $100 \mathrm{ng}$ total RNA, fragmented and labeled ss-cDNA of each sample was hybridized onto a Human Transcriptome Array 2.0 GeneChip ${ }^{\circledR}$ (Affymetrix) [12]. Gene chip data were analyzed using the MADMAX database and analysis pipeline [23]. Probe sets were defined according to Dail et al. [24]. Ranked gene lists created according to the intensity based moderated t-statistics [25] were used as input for Gene Set Enrichment Analysis (GSEA), which was run with 1000 permutations [26, 27]. Functional data analysis was conducted on the filtered data set (interquartile range $>0.2(\log 2)$, intensity $>20$ on at least 5 arrays, $>5$ probes/gene) based upon a false discovery rate (FDR) q-value $<0.05$ testing for time*treatment interactions (EGCG+RES versus PLA). An upstream analysis was performed on the differentially expressed genes $(\mathrm{P}<0.05)$ in AT with Ingenuity Pathway Analysis (June 2015, QIAGEN Silicon Valley, Redwood City, CA, USA).

In addition, we determined gene expression by RT-qPCR for a selection of physiologically significant genes that were most pronounced affected $(\mathrm{FC}>1.25 ; \mathrm{p}<0.05)$ in the microarrays (ATP6V1A, ATP6V1H, CD68, HSL, LAMP2, PI4K2A, UCP2, GAPDH). Reverse transcription of $300 \mathrm{ng}$ of total RNA was performed using iScript cDNA synthesis kit (Bio-Rad), and SYBRGreen based RT-qPCRs were performed using an iCycler (Bio-Rad). Reactions were performed in duplicate on the same plate in a total volume of $25 \mu \mathrm{L}$ containing $5 \mu \mathrm{L}$ 1:6 cDNA, $12.5 \mu \mathrm{L}$ SYBR-Green master mix (IQ SYBR Green Supermix), and gene-specific primers (Biolegio, 
Nijmegen, The Netherlands; primer sequences see Supplemental Table S6.4). Expression was normalized by GAPDH and the $\triangle \triangle \mathrm{CT}$ method was used for calculating relative expression.

Western blot staining was performed in protein lysates from whole fat homogenates after standard SDS-PAGE and Western blotting, using the MitoProfile ${ }^{\circledR}$ Total OXPHOS antibody cocktail (MS601, Mitosciences, Oregon, USA) and an HRP-labeled secondary antibody (P0161, DAKO, Heverlee Belgium). Protein expression values were determined and equally loaded and, as for PCR, standardized against GAPDH protein expression. Protein quantification of oxidative phosphorylation (OxPhos)-complexes was performed by Image Lab ${ }^{\mathrm{TM}}$ Software (V5.2.1. Build 11, BioRad, Veenendaal, the Netherlands).

\section{Statistics}

Data are expressed as mean \pm SEM. Variables were normally distributed. Baseline differences between the EGCG+RES and PLA group were tested by Student's unpaired t-test. The effects of EGCG+RES supplementation compared with PLA were analyzed using a 2-factor (time and treatment) repeated-measures ANOVA. Gene expression data are expressed as fold-changes calculated as the change from baseline for the EGCG+RES group as compared to the change from baseline in the PLA-group: Limma ( $\log 2$ based) Fold Change $=(($ Mean FC: INTV_t3INTV_t0)*(-(Mean FC: PLA_t3-PLA_t0)). Statistics was done using SPSS 19.0 (IBM Corporation, Armonk, NY, USA) for Macintosh. $\mathrm{P}<0.05$ was considered as statistically significant.

\section{Results}

\section{Subjects characteristics}

Twenty-five subjects (10 women, 15 men) were included in the present sub-study, since AT biopsies were not available for all individuals that completed the main trial $(n=38)$. At baseline, subject characteristics and sex-distribution were not significantly different between groups in the total study population [12], and were comparable in the present sub-study (14 PLA; 8 men, 6 women; 11 EGCG+RES, 7 men, 4 women, Table 6.1).

\section{Adipocyte size}

EGCG+RES supplementation had no significant effect on mean adipocyte size or surface area in abdominal subcutaneous AT (Figure 6.1A-B). In line, adipocyte size distribution was unchanged after EGCG+RES supplementation (Figure 6.1C), indicating that EGCG+RES did not induce a shift from large to small adipocytes or vice versa. 
Table 6.1: Subject characteristics and plasma biochemistry

\begin{tabular}{|c|c|c|c|c|c|}
\hline & \multicolumn{2}{|c|}{$\begin{array}{c}\text { PLA } \\
(n=14)\end{array}$} & \multicolumn{2}{|c|}{$\begin{array}{c}\text { EGCG+RES } \\
(n=11)\end{array}$} & \multirow[b]{2}{*}{$P$} \\
\hline & Week 0 & Week 12 & Week 0 & Week 12 & \\
\hline Age, years & $40 \pm 3$ & & $36 \pm 3$ & & \\
\hline $\mathrm{BMI}, \mathrm{kg} / \mathrm{m} 2$ & $29.7 \pm 1.1$ & & $30.5 \pm 0.7$ & & \\
\hline Waist-to-hip-ratio & $0.88 \pm 0.03$ & & $0.88 \pm 0.03$ & & \\
\hline Diastolic BP, mmHg & $111 \pm 3$ & & $119 \pm 2$ & & \\
\hline Systolic BP, mmHg & $74 \pm 2$ & & $76 \pm 2$ & & \\
\hline $\mathrm{HbA} 1 \mathrm{c}, \%$ & $5.15 \pm 0.08$ & & $5.05 \pm 0.06$ & & \\
\hline 2h-Glucose, mmol/l & $5.29 \pm 0.29$ & & $5.19 \pm 0.3$ & & \\
\hline Glucose, $\mathrm{mmol} / \mathrm{l}$ & $5.11 \pm 0.11$ & $5.12 \pm 0.12$ & $5.13 \pm 0.13$ & $5.08 \pm 0.14$ & 0.50 \\
\hline Insulin, mU/l & $9.0 \pm 1.2$ & $10.6 \pm 1.1$ & $8.7 \pm 1.3$ & $7.8 \pm 1.2$ & 0.03 \\
\hline HOMA-IR & $2.03 \pm 0.26$ & $2.39 \pm 0.24$ & $1.97 \pm 0.29$ & $1.77 \pm 0.27$ & 0.03 \\
\hline Free fatty acids, $\mu \mathrm{mol} / \mathrm{l}$ & $532 \pm 32$ & $491 \pm 18$ & $497 \pm 55$ & $532 \pm 53$ & 0.33 \\
\hline Triacylglycerol, mmol/l & $1.06 \pm 0.22$ & $1.31 \pm 0.24$ & $1.75 \pm 0.25$ & $1.93 \pm 0.27$ & 0.65 \\
\hline Adiponectin, $\mu \mathrm{g} / \mathrm{ml}$ & $8.3 \pm 1.0$ & $8.6 \pm 1.0$ & $7.0 \pm 1.1$ & $7.1 \pm 1.1$ & 0.77 \\
\hline Leptin, ng/ml & $23.1 \pm 4.5$ & $24.3 \pm 4.5$ & $17.8 \pm 5.1$ & $16.1 \pm 5.1$ & 0.16 \\
\hline Interleukin-6, pg/ml & $1.00 \pm 0.17$ & $0.91 \pm 0.13$ & $0.69 \pm 0.19$ & $0.77 \pm 0.14$ & 0.46 \\
\hline Interleukin-8, pg/ml & $9.28 \pm 0.94$ & $10.32 \pm 1.06$ & $9.79 \pm 1.07$ & $9.79 \pm 1.20$ & 0.43 \\
\hline TNF-a, pg/ml & $2.86 \pm 0.24$ & $3.3 \pm 0.46$ & $2.87 \pm 0.27$ & $2.81 \pm 0.52$ & 0.26 \\
\hline $\mathrm{EGCG}, \mathrm{ng} / \mathrm{ml}$ & $0 \pm 0$ & $0 \pm 0$ & $0 \pm 0$ & $15 \pm 10$ & $<0.01$ \\
\hline $\mathrm{RES}, \mathrm{ng} / \mathrm{ml}$ & $0 \pm 0$ & $0 \pm 0$ & $0 \pm 0$ & $233 \pm 55$ & $<0.01$ \\
\hline Dihydro-RES, ng/ml & $0 \pm 0$ & $0 \pm 0$ & $0 \pm 0$ & $177 \pm 35$ & $<0.01$ \\
\hline
\end{tabular}

BMI, Body-Mass-Index; BP, blood pressure; HbA1c, glycated hemoglobin A 1c; 2h-glucose, plasma glucose after oral glucosetolerance test; HOMA-IR, Homeostatic Model Assessment of insulin resistance; TNF-a, tumor necrosis factor alpha; EGCG, epigallocatechin-3-gallate; RES, resveratrol. Values given as mean \pm SEM. P, P-value for statistical significance of time*treatment interaction.

\section{Adipose tissue gene expression profile}

Of the 26876 genes on the array, 10987 were analyzed after filtering (Interquartile range $>0.2$ $(\log 2)$, intensity $>20$ on at least 5 arrays, $>5$ probes/gene). 763 genes were differentially expressed after EGCG+RES supplementation as compared to PLA $(\mathrm{P}<0.05)$, of which 424 genes showed higher expression levels (Supplemental Table S6.1).

Using the Databases Kyoto Encyclopedia of Genes and Genomes, Wikipathways, and Biocarta, we identified 332 pathways that were differentially expressed between EGCG+RES and PLA. Strikingly, expression levels of all these pathways were lower after EGCG+RES supplementation as compared to the PLA group, as indicated by negative normalized enrichment scores (NES), whereas no pathways were significantly enriched after EGCC+RES supplementation (Supplemental Table S6.2). More specific, gene sets related to cell turnover (circadian rhythm, cytoskeleton, and apoptosis/autophagy) and transcription and translation showed lower expression levels (Figure 6.2, rows 1-19). Furthermore, EGCG+RES decreased the expression of pathways related to energy and substrate metabolism, oxidative stress, immune defense (Figure 6.2, rows 
20-48) and various diseases, including Alzheimer's, several types of cancer, and infectious, immune, and inflammatory diseases (Supplemental Table S6.2).
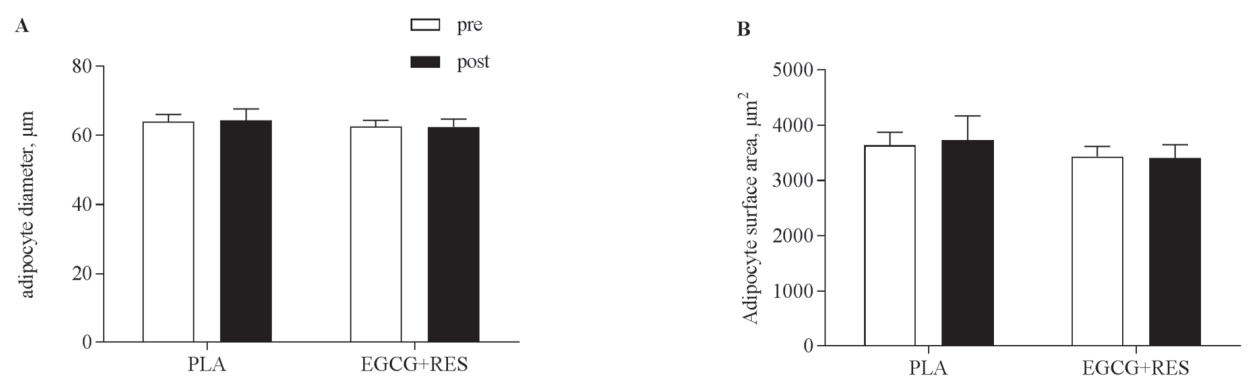

$$
\text { C }
$$

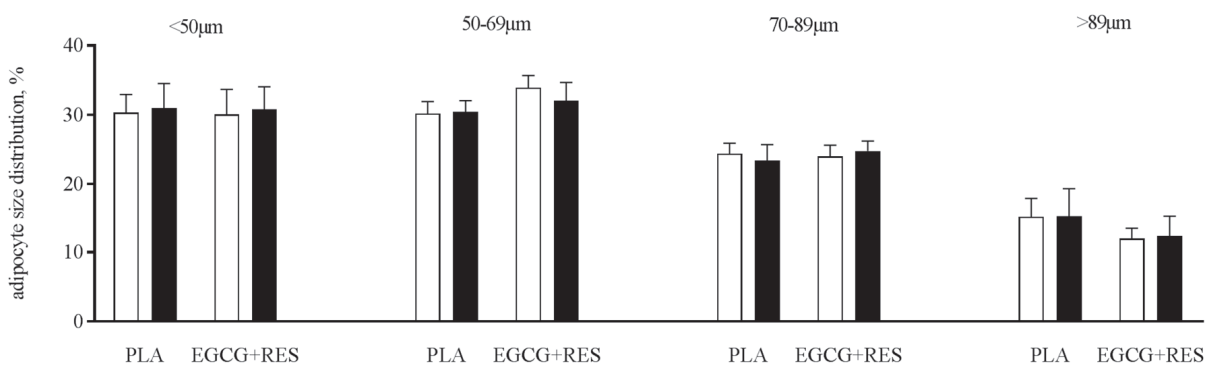

D

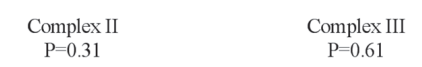

Complex I \& IV

Complex V

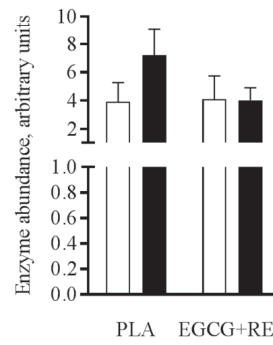

PLA EGCG+RES

PLA EGCG+RES

PLA EGCG+RES

PLA EGCG+RES

Figure 6.1: Abdominal subcutaneous adipocyte morphology and OxPhos protein expression before and after intervention

A) Mean adipocyte diameter, B) surface area, and C) adipocyte size distribution, and D) mitochondrial protein content of complexes I-V were not affected by EGCG+RES supplementation compared with PLA. Open bars: week 0; solid bars: week 12. Statistical significance of time* supplementation interaction indicated as * ${ }^{*}$, when $P<0.05$. Values are given as means \pm SEM $(E G C G+R E S$, $n=5 ;$ PLA $n=9$ ). 
Next, we selected individual genes - by significance $(\mathrm{P}<0.05)$ and fold-change $(\mathrm{FC}>1.25)$ in the microarray analysis - and measured their mRNA expression using quantitative real-time PCR (RTqPCR), with GAPDH as housekeeping gene. We observed no statistically significant differences in mRNA levels between the EGCG+RES and the PLA-group, although the expression pattern (direction of change) was similar for microarray and RT-qPCR results, for all but 2 individual genes (Supplemental Table S6.1).

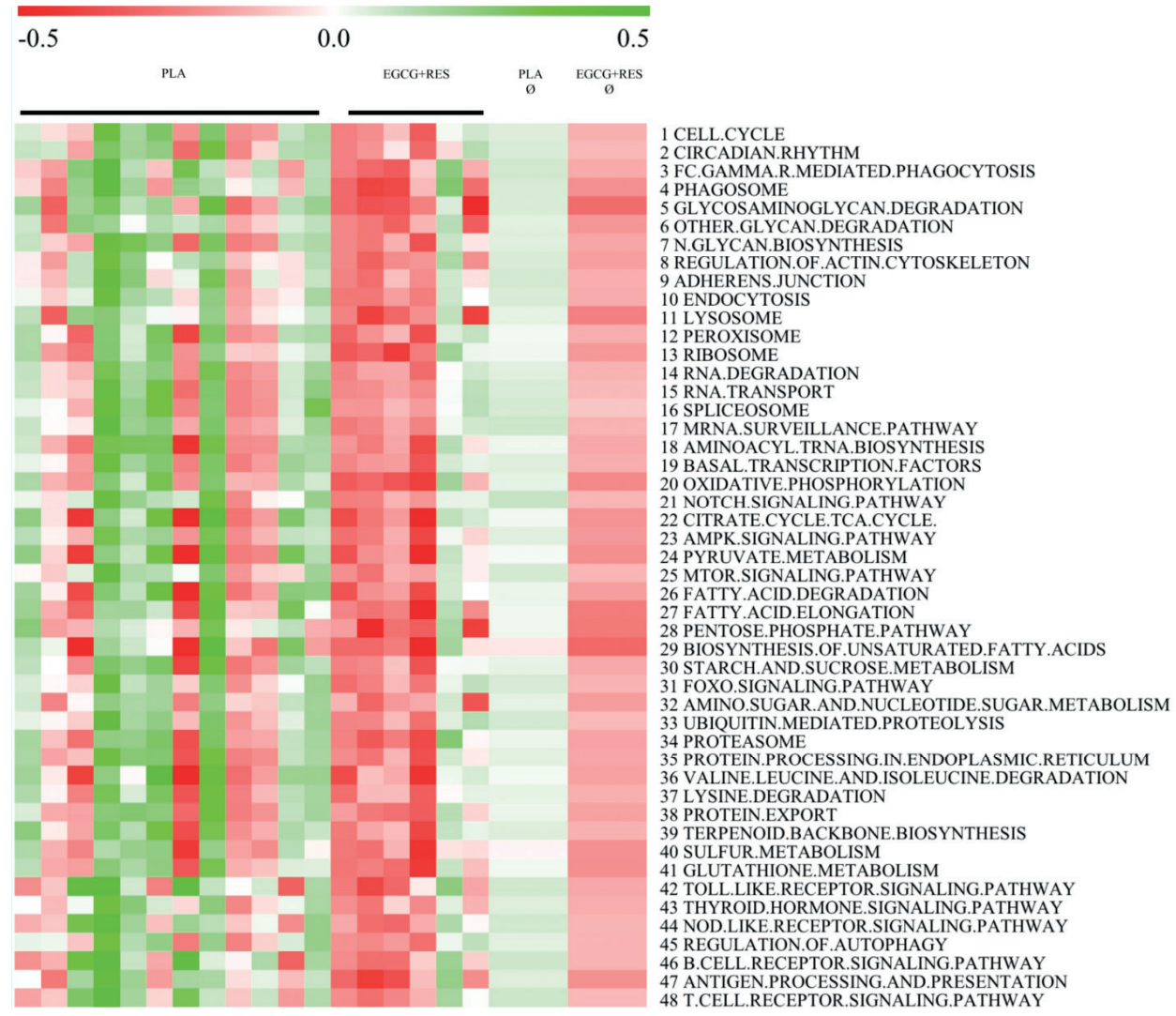

\section{Figure 6.2: Adipose tissue gene expression changes by intervention}

Gene set enrichment analysis (GSEA) of adipose tissue microarray data revealed that gene sets (Kyoto Encyclopedia of Genes, Genomes, Wikipathways, and Biocarta database) related to cell turnover (1-19), energy and substrate metabolism (20-40), inflammation and the immune system (41-48) were significantly downregulated following EGCG+RES supplementation (PLA, $n=12$; $E G C G+R E S, n=6$, data are first presented by individual and in the last two columns as average per group). Red color indicates downregulated pathways, whereas green color indicates upregulated pathways after EGCG+RES versus PLA. Functional data analysis was based upon FDR q-value $<0.05$ on the filtered data set (interquartile range $>0.2$ (log2), intensity $>20$ on at least 5 arrays, $>5$ probes per gene) for the interaction (EGCG+RES versus PLA) with GSEA which was run with 1000 permutations. 
As an indication of macrophage infiltration in the AT, we also measured CD68-expression by RTqPCR. The microarray data showed a significantly lower CD68 expression, indicative for a reduced macrophage infiltration in AT. The RT-qPCR for CD68 expression displayed a tendency in the same direction, but did not reach statistical significance. Possible regulators of adipogenesis ( $\beta$-estradiol, Prolactin), oxidative stress (Genistein, nuclear factor and erythroid 2-like 2 (NRF2)) and inflammation (TNF- $\alpha$ ) were identified by upstream analysis of significantly altered genes $(n=763)$ as potentially inhibited after EGCG+RES supplementation (Supplemental Table S6.3). Therefore, the upstream analysis suggests that EGCG+RES supplementation leads to a decrease in adipogenesis, oxidative stress and inflammation related gene expression. In line, downstreamgene sets regulated by anti-carcinogenic and immune-suppressant drugs were identified as activated and implicate an inhibited proliferation capacity (5-Flourouracil, Trichostatin A, Gentamicin, CD437 and sirolimus).

\section{Adipose tissue oxidative phosphorylation (OxPhos) protein expression}

Protein expression of mitochondrial complex $\mathrm{V}$ tended to be decreased by EGCG+RES supplementation compared with PLA ( $\mathrm{P}=0.11$, Figure 6.1D). Complexes II, III and I+IV were not significantly affected by $\mathrm{EGCG}+\mathrm{RES}(\mathrm{P}=0.31, \mathrm{P}=0.61, \mathrm{P}=0.55$, respectively).

\section{Discussion}

Numerous rodent studies have demonstrated that dietary polyphenols, including EGCG and RES, modulate AT biology $[3,5]$. Here, we demonstrate that supplementation of EGCG+RES for 12 weeks downregulated gene expression of pathways related to adipocyte turnover, energy metabolism, inflammation, and the immune defense. However, EGCG+RES did not induce alterations in adipocyte morphology or OxPhos protein expression in overweight and obese subjects. Importantly, food intake and diet composition did not change throughout the intervention as assessed by 3-day food records [12].

The present study demonstrated that EGCG+RES supplementation decreased the expression of gene sets related to adipogenesis, apoptosis and lipolysis, which are major determinants of adipose tissue and lipid turnover [13-15] and a reduction in both may therefore be indicative of a reduced adipocyte turnover. This, in turn, may have caused the reduced expression of pathways related to substrate metabolism and mitochondrial function, possibly related to lower energy requirements of AT. We acknowledge that other advanced techniques such as isotope dilution may provide more direct evidence of adipose tissue turnover and should be applied in future studies [16]. Importantly, a low turnover rate of adipocytes may reflect a less flexible metabolic phenotype. Indeed, 
decreased adipocyte and lipid turnover has been related to hypertrophic, dysfunctional AT [13], obesity [15] and familial combined hyperlipidemia [14]. Nevertheless, the observed inactivation of pathways corresponding to adipose tissue turnover did not translate into a change in adipocyte size or AT mass in the present study (Figure 6.1). In line with these morphological findings, plasma metabolic profile and whole body, and AT insulin sensitivity did not change significantly after the intervention [12], despite a slight reduction in fasting insulin levels in this subgroup (Table 6.1). At first glance, the present data seem in contrast to a previous study of our group, demonstrating that adipocyte size was significantly reduced, whereas fat mass was unchanged, after 30 days of RES supplementation $(150 \mathrm{mg} / \mathrm{d})$ in obese men [9]. In that study, gene expression and pathway analysis indicated that RES increased adipogenesis and enhanced lysosomal and phagosomal lipid breakdown, which may have contributed to the observed reduction in adipocyte size [9]. Importantly, however, the duration of supplementation, the lower dose of RES and the addition of EGCG as supplement in the present study might explain these opposing findings.

Intriguingly, the present data demonstrate that pathways related to oxidative stress, inflammation and the immune response showed lower expression levels in AT after EGCG+RES intake compared with PLA (Supplemental Table S6.2). These findings are consistent with previous studies showing anti-oxidant and anti-proliferative effects of EGCG and RES, which led to the administration of polyphenols in chemoprevention and cancer therapy [17, 18]. However, due to the importance of oxidative stress and autophagy in cell and tissue homeostasis, cancer development and cardiometabolic complications [19, 20], the (patho)physiological relevance of the present findings over a longer supplementation period remains to be investigated. Contrary to our observations, Konings et al. [9] reported an elevated gene expression for pathways related to immune response. This was interpreted as subsequent effect induced by an increased lysosomal lipid breakdown, and by the decrease in adipocyte size causing traction forces between the adipocytes and their embedding extracellular matrix. As indicated above, duration of supplementation, addition of EGCG supplementation and the inclusion of female subjects may have played a role in these differential findings. Despite the small sample size of this study, which is its major limitation, the results were consistent. If feasible, future studies would benefit from inclusion of a more thorough assessment of macrophage infiltration to characterize the inflammatory status of the AT.

We were not able to confirm the microarray data in a statistically significant way by RT-qPCR of individual genes although the mRNA transcripts followed a similar pattern as our gene expression array data. Over the past decade microarray technology has been established as a sensitive and robust method to detect changes in gene expression patterns [21]. Selection of a handful of genes 
introduces a bias in the analysis that may be avoided by using a transcriptome-wide method. In addition, the GSEA and upstream analysis does not focus on individual genes, but rather on sets of genes, i.e. pathways. Therefore, with the subtle changes we observed for individual genes, potential false positive or false-negative genes have less impact on the overall results using microarray analysis as compared to analyses of individual genes with RT-qPCR.

In conclusion, the present study illustrated that EGCG+RES supplementation for 12 weeks may induce a suppression of gene sets related to adipocyte turnover (adipogenesis and apoptosis/autophagy), inflammation and the immune system in AT in overweight and obese men and women. Although EGCG+RES did not induce any significant effects on body composition, AT morphology, lipolysis, and insulin sensitivity [12], it remains to be determined how the alterations of the AT transcriptome may translate into beneficial changes in metabolic health on longer terms.

\section{Abbreviations}

$\begin{array}{ll}\text { AT } & \text { Adipose tissue } \\ \text { EGCG } & \text { Epigallocatechin-gallate } \\ \text { FDR } & \text { False discovery rate } \\ \text { GSEA } & \text { Gene set enrichment analysis } \\ \text { OxPhos } & \text { Oxidative Phosphorylation } \\ \text { PLA } & \text { Placebo } \\ \text { RES } & \text { Resveratrol }\end{array}$

\section{Acknowledgements}

The authors would like to thank the study participants, as well as Yvonne Essers, Nicole Hoebers, Christine Toepfer, Gabby Hul and Jack Cleutjens for excellent technical support and Pure Encapsulations Inc. for provision of the supplements. This study was funded by the ALPRO foundation. Author contributions: JM, JWEJ, GHG and EEB designed the study. JM performed the experiments and analysed the data. JM, IW, AF, IB, PdG and MVB performed the microarray analysis. JM and IW wrote the manuscript. All authors revised the content of the manuscript, and approved the manuscript for publication. 


\section{References}

1. Lundgren M, Svensson M, Lindmark S, Renstrom F, Ruge T, Eriksson JW. Fat cell enlargement is an independent marker of insulin resistance and 'hyperleptinaemia'. Diabetologia. 2007;50:625-33.

2. Heinonen S, Saarinen L, Naukkarinen J, Rodriguez A, Fruhbeck G, Hakkarainen A, Lundbom J, Lundbom N, Vuolteenaho K, Moilanen E, Arner P, Hautaniemi S, Suomalainen A, Kaprio J, Rissanen A, Pietilainen KH. Adipocyte morphology and implications for metabolic derangements in acquired obesity. Int J Obes (Lond). 2014;38:1423-31.

3. Kim S, Jin Y, Choi Y, Park T. Resveratrol exerts anti-obesity effects via mechanisms involving downregulation of adipogenic and inflammatory processes in mice. Biochem Pharmacol. 2011;81:134351.

4. Lagouge M, Argmann C, Gerhart-Hines Z, Meziane H, Lerin C, Daussin F, Messadeq N, Milne J, Lambert P, Elliott P, Geny B, Laakso M, Puigserver P, Auwerx J. Resveratrol improves mitochondrial function and protects against metabolic disease by activating SIRT1 and PGC-1alpha. Cell. 2006;127:1109-22. Epub 2006/11/23.

5. Lee MS, Kim CT, Kim Y. Green tea (-)-epigallocatechin-3-gallate reduces body weight with regulation of multiple genes expression in adipose tissue of diet-induced obese mice. Ann Nutr Metab. 2009;54:151-7.

6. Wolfram S, Raederstorff D, Wang Y, Teixeira SR, Elste V, Weber P. TEAVIGO (epigallocatechin gallate) supplementation prevents obesity in rodents by reducing adipose tissue mass. Ann Nutr Metab. 2005;49:54-63.

7. Yoshino J, Conte C, Fontana L, Mittendorfer B, Imai S, Schechtman KB, Gu C, Kunz I, Rossi Fanelli F, Patterson BW, Klein S. Resveratrol supplementation does not improve metabolic function in nonobese women with normal glucose tolerance. Cell Metab. 2012;16:658-64.

8. Mielgo-Ayuso J, Barrenechea L, Alcorta P, Larrarte E, Margareto J, Labayen I. Effects of dietary supplementation with epigallocatechin-3-gallate on weight loss, energy homeostasis, cardiometabolic risk factors and liver function in obese women: randomised, double-blind, placebo-controlled clinical trial. Br J Nutr. 2014;111:1263-71.

9. Konings E, Timmers S, Boekschoten MV, Goossens GH, Jocken JW, Afman LA, Muller M, Schrauwen P, Mariman EC, Blaak EE. The effects of 30 days resveratrol supplementation on adipose tissue morphology and gene expression patterns in obese men. Int J Obes (Lond). 2014;38:470-3.

10. Most J, van Can JG, van Dijk JW, Goossens GH, Jocken J, Hospers JJ, Bendik I, Blaak EE. A 3-day EGCG-supplementation reduces interstitial lactate concentration in skeletal muscle of overweight subjects. Sci Rep. 2015;5:17896.

11. Most J, Goossens GH, Jocken JW, Blaak EE. Short-term supplementation with a specific combination of dietary polyphenols increases energy expenditure and alters substrate metabolism in overweight subjects. Int J Obes (Lond). 2014;38:698-706.

12. Most J, Timmers S, Warnke I, Jocken JW, van Boekschoten M, de Groot P, Bendik I, Schrauwen P, Goossens GH, Blaak EE. Combined epigallocatechin-3-gallate and resveratrol supplementation for $12 \mathrm{wk}$ increases mitochondrial capacity and fat oxidation, but not insulin sensitivity, in obese humans: a randomized controlled trial. Am J Clin Nutr. 2016;104:215-27. Epub 2016/05/20.

13. Arner E, Westermark PO, Spalding KL, Britton T, Ryden M, Frisen J, Bernard S, Arner P. Adipocyte turnover: relevance to human adipose tissue morphology. Diabetes. 2010;59:105-9.

14. Arner P, Bernard S, Salehpour M, Possnert G, Liebl J, Steier P, Buchholz BA, Eriksson M, Arner E, Hauner H, Skurk T, Ryden M, Frayn KN, Spalding KL. Dynamics of human adipose lipid turnover in health and metabolic disease. Nature. 2011;478:110-3.

15. Ryden M, Andersson DP, Bernard S, Spalding K, Arner P. Adipocyte triglyceride turnover and lipolysis in lean and overweight subjects. J Lipid Res. 2013;54:2909-13.

16. White UA, Tchoukalova YD. Implications of $2 \mathrm{H}$-labeling of DNA protocol to measure in vivo cell turnover in adipose tissue. Adipocyte. 2012;1:242-5.

17. Goswami SK, Das DK. Resveratrol and chemoprevention. Cancer Lett. 2009;284:1-6.

18. Yang CS, Wang X, Lu G, Picinich SC. Cancer prevention by tea: animal studies, molecular mechanisms and human relevance. Nat Rev Cancer. 2009;9:429-39.

19. Kim KH, Lee MS. Autophagy--a key player in cellular and body metabolism. Nat Rev Endocrinol. 2014;10:322-37. 
20. Gorrini C, Harris IS, Mak TW. Modulation of oxidative stress as an anticancer strategy. Nat Rev Drug Discov. 2013;12:931-47.

21. Morey JS, Ryan JC, Van Dolah FM. Microarray validation: factors influencing correlation between oligonucleotide microarrays and real-time PCR. Biol Proced Online. 2006;8:175-93.

22. Goossens GH, Bizzarri A, Venteclef N, Essers Y, Cleutjens JP, Konings E, Jocken JW, Cajlakovic M, Ribitsch V, Clement K, Blaak EE. Increased adipose tissue oxygen tension in obese compared with lean men is accompanied by insulin resistance, impaired adipose tissue capillarization, and inflammation. Circulation. 2011;124:67-76.

23. Lin K, Kools H, de Groot PJ, Gavai AK, Basnet RK, Cheng F, Wu J, Wang X, Lommen A, Hooiveld GJ, Bonnema G, Visser RG, Muller MR, Leunissen JA. MADMAX - Management and analysis database for multiple omics experiments. J Integr Bioinform. 2011;8:160.

24. Dai MH, Wang PL, Boyd AD, Kostov G, Athey B, Jones EG, Bunney WE, Myers RM, Speed TP, Akil H, Watson SJ, Meng F. Evolving gene/transcript definitions significantly alter the interpretation of GeneChip data. Nucleic Acids Research. 2005;33.

25. Sartor MA, Tomlinson CR, Wesselkamper SC, Sivaganesan S, Leikauf GD, Medvedovic M. Intensity-based hierarchical Bayes method improves testing for differentially expressed genes in microarray experiments. Bmc Bioinformatics. 2006;7.

26. Subramanian A, Tamayo P, Mootha VK, Mukherjee S, Ebert BL, Gillette MA, Paulovich A, Pomeroy SL, Golub TR, Lander ES, Mesirov JP. Gene set enrichment analysis: A knowledge-based approach for interpreting genome-wide expression profiles. Proceedings of the National Academy of Sciences of the United States of America. 2005;102:15545-50.

27. Mootha VK, Lindgren CM, Eriksson KF, Subramanian A, Sihag S, Lehar J, Puigserver P, Carlsson E, Ridderstrale M, Laurila E, Houstis N, Daly MJ, Patterson N, Mesirov JP, Golub TR, Tamayo P, Spiegelman B, Lander ES, Hirschhorn JN, Altshuler D, Groop LC. PGC-1 alpha-responsive genes involved in oxidative phosphorylation are coordinately downregulated in human diabetes. Nature Genetics. 2003;34:267-73. 
Supplemental Material 


\section{Supplemental Results}

\section{Supplemental Table S6.1: Condensed selection of most up- and downregulated genes} $(n=25$, inclusive and plus genes analyzed with $R T-q P C R)$

\begin{tabular}{|c|c|c|c|c|c|c|c|}
\hline & Gene & Entrez ID & Description & FC & $\mathbf{P}$ & FC & $\mathbf{P}$ \\
\hline \multicolumn{4}{|c|}{ Most downregulated, sorted by FC } & \multicolumn{2}{|c|}{ Micro-array } & \multicolumn{2}{|c|}{ RT-qPCR } \\
\hline 1 & MS4A6E & 245802 & $\begin{array}{l}\text { membrane-spanning 4-domains, } \\
\text { subfamily } A \text {, member } 6 E\end{array}$ & -2.042 & 0.031 & & \\
\hline 2 & GPR183 & 1880 & G protein-coupled receptor 183 & -1.620 & 0.006 & & \\
\hline 3 & SPESP1 & 246777 & sperm equatorial segment protein 1 & -1.494 & 0.004 & & \\
\hline 4 & RNASE6 & 6039 & ribonuclease, RNase A family, $\mathrm{k} 6$ & -1.489 & 0.026 & & \\
\hline 5 & LOC101927405 & 101927405 & uncharacterized LOC101927405 & -1.482 & 0.020 & & \\
\hline 6 & CD68 & 968 & CD68 molecule & -1.455 & 0.041 & -1.033 & 0.779 \\
\hline 7 & LOC102723793 & 102723793 & uncharacterized LOC102723793 & -1.429 & 0.023 & & \\
\hline 8 & LOC102724528 & 102724528 & uncharacterized LOC102724528 & -1.420 & 0.020 & & \\
\hline 9 & GPNMB & 10457 & glycoprotein (transmembrane) nmb & -1.412 & 0.050 & & \\
\hline 10 & SLMO2 & 51012 & slowmo homolog 2 (Drosophila) & -1.411 & 0.046 & & \\
\hline 11 & CXCL16 & 58191 & chemokine (C-X-C motif) ligand 16 & -1.409 & 0.023 & & \\
\hline 12 & UCP2 & 7351 & $\begin{array}{l}\text { uncoupling protein } 2 \text { (mitochondrial, } \\
\text { proton carrier) }\end{array}$ & -1.400 & 0.004 & -1.347 & 0.655 \\
\hline 13 & CTSD & 1509 & cathepsin D & -1.399 & 0.025 & & \\
\hline 14 & PRDX3 & 10935 & peroxiredoxin 3 & -1.395 & 0.010 & & \\
\hline 15 & LOC101929216 & 101929216 & uncharacterized LOC101929216 & -1.394 & 0.047 & & \\
\hline 16 & SLC31A1 & 1317 & $\begin{array}{l}\text { solute carrier family } 31 \text { (copper } \\
\text { transporter), member } 1\end{array}$ & -1.393 & 0.005 & & \\
\hline 17 & GPRIN3 & 285513 & GPRIN family member 3 & -1.388 & 0.003 & & \\
\hline 18 & TMSB4X & 7114 & thymosin beta $4, \mathrm{X}$-linked & -1.375 & 0.004 & & \\
\hline 19 & SAMHD1 & 25939 & SAM domain and HD domain 1 & -1.375 & 0.040 & & \\
\hline 20 & MS4A7 & 58475 & $\begin{array}{l}\text { membrane-spanning 4-domains, } \\
\text { subfamily } A \text {, member } 7\end{array}$ & -1.373 & 0.029 & & \\
\hline 21 & S100A4 & 6275 & S100 calcium binding protein $\mathrm{A} 4$ & -1.368 & 0.019 & & \\
\hline 22 & MMP19 & 4327 & matrix metallopeptidase 19 & -1.366 & 0.039 & & \\
\hline 23 & MIR4435-1HG & 541471 & $\begin{array}{l}\text { MIR4435-1 host gene (non-protein } \\
\text { coding) }\end{array}$ & -1.364 & 0.018 & & \\
\hline 24 & RPL23A & 6147 & ribosomal protein L23a & -1.363 & 0.028 & & \\
\hline 25 & IFl6 & 2537 & interferon, alpha-inducible protein 6 & -1.360 & 0.039 & & \\
\hline \multicolumn{8}{|c|}{ Additional genes selected by hypothesis to physiological relevance } \\
\hline & $\mathrm{PI} 4 \mathrm{~K} 2 \mathrm{~A}$ & 55361 & $\begin{array}{l}\text { phosphatidylinositol 4-kinase type } 2 \\
\text { alpha }\end{array}$ & -1.283 & 0.001 & -1.377 & 0.600 \\
\hline & LAMP2 & 3920 & $\begin{array}{l}\text { lysosomal-associated membrane } \\
\text { protein } 2\end{array}$ & -1.279 & 0.008 & -2.034 & 0.111 \\
\hline & LIPE & 3991 & lipase, hormone-sensitive & -1.271 & 0.019 & 1.155 & 0.576 \\
\hline & ATP6V1A & 523 & $\begin{array}{l}\text { ATPase, } \mathrm{H}+\text { transporting, lysosomal } \\
70 \mathrm{kDa}, \mathrm{V} 1 \text { subunit } \mathrm{A}\end{array}$ & -1.255 & 0.009 & -1.079 & 0.560 \\
\hline & ATP6V1H & 51606 & $\begin{array}{l}\text { ATPase, } \mathrm{H}+\text { transporting, lysosomal } \\
50 / 57 \mathrm{kDa}, \mathrm{V} 1 \text { subunit } \mathrm{H}\end{array}$ & -1.250 & 0.004 & -1.074 & 0.982 \\
\hline \multicolumn{8}{|c|}{ Most upregulated, sorted by FC } \\
\hline 1 & RNU6-23P & 100873755 & $\begin{array}{l}\text { RNA, U6 small nuclear 23, } \\
\text { pseudogene }\end{array}$ & 2.057 & 0.013 & & \\
\hline 2 & MIR6875 & 102466755 & microRNA 6875 & 1.790 & 0.002 & & \\
\hline 3 & MIR4442 & 100616477 & microRNA 4442 & 1.688 & 0.004 & & \\
\hline 4 & MIR3689D2 & 100616344 & microRNA 3689d-2 & 1.623 & 0.030 & & \\
\hline 5 & TRM-CAT1-1 & 100189201 & transfer RNA-Met (CAT) 1-1 & 1.611 & 0.011 & & \\
\hline 6 & MIR6873 & 102466754 & microRNA 6873 & 1.590 & 0.004 & & \\
\hline
\end{tabular}




\begin{tabular}{|l|l|l|l|l|l|l|l|}
\hline 7 & TRS-AGA3-1 & 790951 & transfer RNA-Ser (AGA) 3-1 & 1.583 & 0.011 & & \\
\hline 8 & SNORA71E & 677821 & small nucleolar RNA, H/ACA box 71E & 1.571 & 0.010 & & \\
\hline 9 & $\begin{array}{l}\text { RNASEK- } \\
\text { C17orf49 }\end{array}$ & 100529209 & RNASEK-C17orf49 readthrough & 1.565 & 0.003 & & \\
\hline 10 & MIR936 & 100126326 & microRNA 936 & 1.547 & 0.001 & & \\
\hline 11 & TRM-CAT6-1 & 100189226 & transfer RNA-Met (CAT) 6-1 & 1.520 & 0.025 & & \\
\hline 12 & ERCC6-PGBD3 & 101243544 & ERCC6-PGBD3 readthrough & 1.513 & 0.006 & & \\
\hline 13 & MIR133B & 442890 & microRNA 133b & 1.505 & 0.002 & & \\
\hline 14 & TRK-CTT6-1 & 100188995 & transfer RNA-Lys (CTT) 6-1 & 1.500 & 0.044 & & \\
\hline 15 & CT47B1 & 643311 & $\begin{array}{l}\text { cancer/testis antigen family 47, } \\
\text { member B1 }\end{array}$ & 1.498 & 0.001 & & \\
\hline 16 & LOC728554 & 728554 & THO complex 3 pseudogene & 1.485 & 0.007 & & \\
\hline 17 & LOC101929974 & 101929974 & uncharacterized LOC101929974 & 1.481 & 0.008 & & \\
\hline 18 & TRS-CGA4-1 & 100189152 & transfer RNA-Ser (CGA) 4-1 & 1.476 & 0.014 & & \\
\hline 19 & IGLJ4 & 28830 & $\begin{array}{l}\text { immunoglobulin lambda joining 4 (non- } \\
\text { functional) }\end{array}$ & 1.474 & 0.015 & & \\
\hline 20 & MIR3147 & 100422939 & microRNA 3147 & 1.473 & 0.010 & & \\
\hline 21 & LOC729468 & 729468 & putative PGM5-like protein 1 & 1.463 & 0.007 & & \\
\hline 22 & TRAJ7 & 28748 & T cell receptor alpha joining 7 & 1.455 & 0.007 & & \\
\hline 23 & MIR1292 & 100302138 & microRNA 1292 & 1.448 & 0.033 & & \\
\hline 24 & MIR1185-2 & 100302209 & microRNA 1185-2 & 1.438 & 0.010 & & \\
\hline 25 & TRG-CCC5-1 & 100189045 & transfer RNA-Gly (CCC) 5-1 & 1.431 & 0.012 & & \\
\hline
\end{tabular}

Shortened list of the most up- and downregulated genes as analyzed by micro-array (both, $n=25$ ) and additional genes ( $n=7$ ) based on their physiological relevance to our hypothesis analyzed by RT-qPCR. Fold-changes are calculated as fold-change from baseline for the EGCG+RES group as compared to the fold-change from baseline in the PLA-group, and P-values are raw $p$ values from regularized paired t-test $\left(F C(E G C G+R E S){ }^{*} F C(P L A)\right)$.

Supplemental Table S6.2: Condensed list of up- and downregulated pathways (NES $<-2.0)$

\begin{tabular}{|c|c|c|c|}
\hline Pathway & NES & $\begin{array}{l}\text { q- } \\
\text { value }\end{array}$ & Source \\
\hline \multicolumn{4}{|l|}{ Cell cycle } \\
\hline APC.C.MEDIATED.DEGRADATION.OF.CELL.CYCLE.PROTEINS & -2.85 & 0.000 & WP1782 \\
\hline CELL.CYCLE & -2.36 & 0.000 & KEGG \\
\hline CELL.CYCLE.CHECKPOINTS & -3.02 & 0.000 & WP1775 \\
\hline EGF.EGFR.SIGNALING.PATHWAY & -2.31 & 0.000 & WP437 \\
\hline EUKARYOTIC.TRANSCRIPTION.INITIATION & -2.48 & 0.000 & WP405 \\
\hline G13.SIGNALING.PATHWAY & -2.32 & 0.000 & WP524 \\
\hline M.G1.TRANSITION & -2.80 & 0.000 & WP2785 \\
\hline MITOTIC.G1.G1.S.PHASES & -2.67 & 0.000 & WP1858 \\
\hline MITOTIC.METAPHASE.AND.ANAPHASE & -2.85 & 0.000 & WP2757 \\
\hline MITOTIC.PROPHASE & -2.50 & 0.000 & WP2654 \\
\hline S.PHASE & -2.74 & 0.000 & WP2772 \\
\hline \multicolumn{4}{|l|}{ Cellular maintenance } \\
\hline ANDROGEN.RECEPTOR.SIGNALING.PATHWAY & -2.51 & 0.000 & WP138 \\
\hline CIRCADIAN.RHYTHM & -2.29 & 0.000 & KEGG \\
\hline DIURNALLY.REGULATED.GENES.WITH.CIRCADIAN.ORTHOLOGS & -2.25 & 0.000 & WP410 \\
\hline FCGAMMA.RECEPTOR.FCGR.DEPENDENT.PHAGOCYTOSIS. & -2.23 & 0.000 & WP2719 \\
\hline PHAGOSOME & -2.79 & 0.000 & KEGG \\
\hline OTHER.GLYCAN.DEGRADATION & -2.06 & 0.002 & KEGG \\
\hline MEMBRANE.TRAFFICKING & -2.67 & 0.000 & WP1846 \\
\hline N.GLYCAN.BIOSYNTHESIS & -2.18 & 0.001 & KEGG \\
\hline CDC42RAC & -2.16 & 0.001 & $\mathrm{BIOC}$ \\
\hline SPHINGOLIPID.METABOLISM & -2.28 & 0.000 & WP2788 \\
\hline ADHERENS.JUNCTION & -2.28 & 0.000 & KEGG \\
\hline LYSOSOME & -3.58 & 0.000 & KEGG \\
\hline
\end{tabular}


Dietary polyphenols and adipose tissue

\begin{tabular}{|c|c|c|c|}
\hline \multicolumn{4}{|l|}{ Transcription \& Translation } \\
\hline RIBOSOME & -2.39 & 0.000 & KEGG \\
\hline DEADENYLATION.DEPENDENT.MRNA.DECAY & -2.14 & 0.001 & WP2659 \\
\hline HISTONE.MODIFICATIONS & -2.42 & 0.000 & WP2369 \\
\hline METABOLISM.OF.NON.CODING.RNA & -2.10 & 0.001 & WP2715 \\
\hline MRNA.CAPPING & -2.22 & 0.000 & WP1861 \\
\hline RNA.DEGRADATION & -2.39 & 0.000 & KEGG \\
\hline MRNA.PROCESSING & -2.14 & 0.001 & WP411 \\
\hline RNA.TRANSPORT & -2.55 & 0.000 & KEGG \\
\hline PROCESSING.OF.CAPPED.INTRON.CONTAINING.PRE.MRNA & -2.51 & 0.000 & WP1889 \\
\hline SPLICEOSOME & -2.19 & 0.000 & KEGG \\
\hline REGULATION.OF.DNA.REPLICATION & -2.81 & 0.000 & WP1898 \\
\hline REGULATION.OF.MRNA.STABILITY.BY.PROTEINS.THAT.BIND.AU.RICH.ELEMENTS & -3.00 & 0.000 & WP2733 \\
\hline REGULATORY.RNA.PATHWAYS & -2.30 & 0.000 & WP1901 \\
\hline MRNA.SURVEILLANCE.PATHWAY & -2.25 & 0.000 & KEGG \\
\hline RNA.POLYMERASE.II.TRANSCRIPTION & -2.46 & 0.000 & WP1906 \\
\hline SRP.DEPENDENT.COTRANSLATIONAL.PROTEIN.TARGETING.TO.MEMBRANE & -2.36 & 0.000 & WP2737 \\
\hline SYNTHESIS.OF.DNA & -2.68 & 0.000 & WP1925 \\
\hline TRANSCRIPTIONAL.REGULATION.OF.WHITE.ADIPOCYTE.DIFFERENTIATION & -2.23 & 0.000 & WP2751 \\
\hline \multicolumn{4}{|l|}{ Metabolism mitochondria } \\
\hline ELECTRON.TRANSPORT.CHAIN & -2.81 & 0.000 & WP111 \\
\hline ENERGY.METABOLISM & -2.29 & 0.000 & WP1541 \\
\hline NOTCH.SIGNALING.PATHWAY & -2.14 & 0.001 & WP61 \\
\hline OXIDATIVE.PHOSPHORYLATION & -2.79 & 0.000 & KEGG \\
\hline RESPIRATORY.ELECTRON.TRANSPORT.ATP.SYNTHESIS.BY.CHEMIOSMOTIC.COU & -2.58 & 0.000 & WP1902 \\
\hline PLING.AND.HEAT.PRODUCTION.BY.UNCOUPLING.PROTEINS. & & & \\
\hline SIGNALING.BY.NOTCH1 & -2.18 & 0.001 & WP2720 \\
\hline NOTCH.SIGNALING.PATHWAY & -2.11 & 0.001 & KEGG \\
\hline CITRATE.CYCLE.TCA.CYCLE. & -2.24 & 0.000 & KEGG \\
\hline THE.CITRIC.ACID.TCA.CYCLE.AND.RESPIRATORY.ELECTRON.TRANSPORT. & -2.61 & 0.000 & WP2766 \\
\hline \multicolumn{4}{|l|}{ Metabolism Protein/AA } \\
\hline DEGRADATION.OF.BETA.CATENIN.BY.THE.DESTRUCTION.COMPLEX & -2.72 & 0.000 & WP2773 \\
\hline METABOLISM.OF.AMINO.ACIDS.AND.DERIVATIVES & -2.34 & 0.000 & WP2693 \\
\hline PROTEASOME.DEGRADATION & -2.78 & 0.000 & WP183 \\
\hline PROTEASOME & -2.42 & 0.000 & $\mathrm{BIOC}$ \\
\hline PROTEIN.PROCESSING.IN.ENDOPLASMIC.RETICULUM & -3.09 & 0.000 & KEGG \\
\hline VALINE.LEUCINE.AND.ISOLEUCINE.DEGRADATION & -2.15 & 0.001 & KEGG \\
\hline LYSINE.DEGRADATION & -2.14 & 0.001 & KEGG \\
\hline PROTEIN.EXPORT & -2.37 & 0.000 & KEGG \\
\hline \multicolumn{4}{|l|}{ Metabolism substrate \& energy } \\
\hline AMPK.SIGNALING.PATHWAY & -2.17 & 0.001 & KEGG \\
\hline PYRUVATE.METABOLISM & -2.21 & 0.000 & KEGG \\
\hline FATTY.ACID.BETA.OXIDATION & -2.05 & 0.002 & WP143 \\
\hline FATTY.ACID.BIOSYNTHESIS & -2.31 & 0.000 & WP357 \\
\hline FATTY.ACID.ELONGATION & -2.19 & 0.000 & KEGG \\
\hline FATTY.ACID.TRIACYLGLYCEROL.AND.KETONE.BODY.METABOLISM & -2.25 & 0.000 & WP1817 \\
\hline MAPK.SIGNALING.PATHWAY & -2.08 & 0.002 & WP382 \\
\hline METABOLISM.OF.CARBOHYDRATES & -2.08 & 0.001 & WP1848 \\
\hline PENTOSE.PHOSPHATE.PATHWAY & -2.23 & 0.000 & KEGG \\
\hline REGULATION.OF.LIPID.METABOLISM.BY.PEROXISOME.PROLIFERATOR.ACTIVATED & -2.24 & 0.000 & WP2797 \\
\hline .RECEPTOR.ALPHA.PPARALPHA. & & & \\
\hline TRANSLOCATION.OF.GLUT4.TO.THE.PLASMA.MEMBRANE & -2.31 & 0.000 & WP2777 \\
\hline BIOSYNTHESIS.OF.UNSATURATED.FATTY.ACIDS & -2.04 & 0.002 & KEGG \\
\hline \multicolumn{4}{|l|}{ Cholesterol \& Micronutrients } \\
\hline IRON.UPTAKE.AND.TRANSPORT & -2.15 & 0.001 & WP2670 \\
\hline REGULATION.OF.CHOLESTEROL.BIOSYNTHESIS.BY.SREBP.SREBF. & -2.10 & 0.001 & WP2686 \\
\hline TERPENOID.BACKBONE.BIOSYNTHESIS & -2.11 & 0.001 & KEGG \\
\hline SREBP.SIGNALLING & -2.23 & 0.000 & WP1982 \\
\hline \multicolumn{4}{|l|}{ Oxidative stress } \\
\hline DETOXIFICATION.OF.REACTIVE.OXYGEN & -2.22 & 0.000 & WP2824 \\
\hline
\end{tabular}




\begin{tabular}{|c|c|c|c|}
\hline $\begin{array}{l}\text { FOXO.SIGNALING.PATHWAY } \\
\text { GLUTATHIONE.METABOLISM } \\
\text { OXIDATIVE.STRESS }\end{array}$ & $\begin{array}{l}-2.14 \\
-2.18 \\
-2.12 \\
\end{array}$ & $\begin{array}{l}0.001 \\
0.001 \\
0.001 \\
\end{array}$ & $\begin{array}{l}\text { KEGG } \\
\text { KEGG } \\
\text { WP408 }\end{array}$ \\
\hline \multicolumn{4}{|l|}{ Inflammation, Immune system } \\
\hline INTERFERON.GAMMA.SIGNALING & -2.39 & 0.000 & WP1836 \\
\hline MYD88.MAL.CASCADE.INITIATED.ON.PLASMA.MEMBRANE & -2.28 & 0.000 & WP2761 \\
\hline TNF.ALPHA.SIGNALING.PATHWAY & -2.18 & 0.000 & WP2808 \\
\hline TOLL.LIKE.RECEPTOR.SIGNALING.PATHWAY & -2.26 & 0.000 & WP75 \\
\hline TYPE.II.INTERFERON.SIGNALING.IFNG. & -2.31 & 0.000 & WP619 \\
\hline AGE.RAGE.PATHWAY & -2.11 & 0.001 & WP2324 \\
\hline ASPARAGINE.N.LINKED.GLYCOSYLATION & -2.69 & 0.000 & WP1785 \\
\hline B.CELL.RECEPTOR.SIGNALING.PATHWAY & -2.03 & 0.002 & WP23 \\
\hline CLASS.I.MHC.MEDIATED.ANTIGEN.PROCESSING.AMP.PRESENTATION & -3.07 & 0.000 & WP2796 \\
\hline ANTIGEN.PROCESSING.AND.PRESENTATION & -2.79 & 0.000 & KEGG \\
\hline COSTIMULATION.BY.THE.CD28.FAMILY & -2.13 & 0.001 & WP1799 \\
\hline MHC.CLASS.II.ANTIGEN.PRESENTATION & -2.81 & 0.000 & WP2679 \\
\hline SIGNALING.BY.THE.B.CELL.RECEPTOR.BCR. & -2.49 & 0.000 & WP2746 \\
\hline TCR.SIGNALING & -2.30 & 0.000 & WP1927 \\
\hline \multicolumn{4}{|l|}{ Autophagy/apoptosis } \\
\hline APOPTOSIS.MODULATION.BY.HSP70 & -1.89 & 0.008 & WP384 \\
\hline REGULATION.OF.APOPTOSIS & -2.62 & 0.000 & WP1896 \\
\hline SIGNALING.BY.EGFR & -2.48 & 0.000 & WP1910 \\
\hline \multicolumn{4}{|l|}{ Infection, disease, cancer } \\
\hline ALLOGRAFT.REJECTION & -2.33 & 0.000 & KEGG \\
\hline ALZHEIMER.S.DISEASE & -2.34 & 0.000 & KEGG \\
\hline ASTHMA & -2.11 & 0.001 & KEGG \\
\hline AUTOIMMUNE.THYROID.DISEASE & -2.16 & 0.001 & KEGG \\
\hline CHAGAS.DISEASE.AMERICAN.TRYPANOSOMIASIS. & -2.10 & 0.001 & KEGG \\
\hline EPITHELIAL.CELL.SIGNALING.IN.HELICOBACTER.PYLORI.INFECTION & -2.29 & 0.000 & KEGG \\
\hline EPSTEIN.BARR.VIRUS.INFECTION & -2.69 & 0.000 & KEGG \\
\hline SIGNALING.PATHWAYS.IN.GLIOBLASTOMA & -2.14 & 0.001 & WP2261 \\
\hline GRAFT.VERSUS.HOST.DISEASE & -2.37 & 0.000 & KEGG \\
\hline HEPATITIS.B & -2.31 & 0.000 & KEGG \\
\hline HEPATITIS.C & -2.22 & 0.000 & KEGG \\
\hline HERPES.SIMPLEX.INFECTION & -2.92 & 0.000 & KEGG \\
\hline HIV.LIFE.CYCLE & -2.93 & 0.000 & WP2658 \\
\hline HOST.INTERACTIONS.OF.HIV.FACTORS & -3.02 & 0.000 & WP2684 \\
\hline HUNTINGTON.S.DISEASE & -2.56 & 0.000 & KEGG \\
\hline INFLAMMATORY.BOWEL.DISEASE.IBD. & -2.16 & 0.001 & KEGG \\
\hline INFLUENZA.A & -2.97 & 0.000 & KEGG \\
\hline INFLUENZA.LIFE.CYCLE & -2.47 & 0.000 & WP2683 \\
\hline INTEGRATED.BREAST.CANCER.PATHWAY & -2.33 & 0.000 & WP1984 \\
\hline LATENT.INFECTION.OF.HOMO.SAPIENS.WITH.MYCOBACTERIUM.TUBERCULOSIS & -2.24 & 0.000 & WP2700 \\
\hline LEGIONELLOSIS & -2.30 & 0.000 & KEGG \\
\hline LEISHMANIASIS & -2.36 & 0.000 & KEGG \\
\hline MEASLES & -2.79 & 0.000 & KEGG \\
\hline NON.ALCOHOLIC.FATTY.LIVER.DISEASE.NAFLD. & -2.12 & 0.001 & KEGG \\
\hline PARKINSON.S.DISEASE & -2.33 & 0.000 & KEGG \\
\hline RHEUMATOID.ARTHRITIS & -2.53 & 0.000 & KEGG \\
\hline SALMONELLA.INFECTION & -2.19 & 0.000 & KEGG \\
\hline STAPHYLOCOCCUS.AUREUS.INFECTION & -2.54 & 0.000 & KEGG \\
\hline SYSTEMIC.LUPUS.ERYTHEMATOSUS & -2.68 & 0.000 & KEGG \\
\hline TOXOPLASMOSIS & -2.52 & 0.000 & KEGG \\
\hline TUBERCULOSIS & -2.68 & 0.000 & KEGG \\
\hline TYPE.I.DIABETES.MELLITUS & -2.17 & 0.001 & KEGG \\
\hline VIBRIO.CHOLERAE.INFECTION & -2.65 & 0.000 & KEGG \\
\hline VIRAL.CARCINOGENESIS & -2.60 & 0.000 & KEGG \\
\hline VIRAL.MYOCARDITIS & -2.67 & 0.000 & KEGG \\
\hline
\end{tabular}


NES, normalized enrichment score; q, statistical significance of false discovery rate (FDR) of post versus pre-enrichment comparing changes after EGCG+RES versus changes after placebo; Source represents the database, in which the respective pathway has been identified.

\section{Supplemental Table S6.3: Ingenuity upstream analysis including related genes}

\begin{tabular}{|c|c|c|c|c|c|}
\hline & Upstream regulator & $\begin{array}{l}\text { Downstream targets } \\
\text { /pathways }\end{array}$ & & Genes & $\mathbf{P}$ \\
\hline \multicolumn{6}{|c|}{ Adipogenesis } \\
\hline Inhibited & $\beta$-Estradiol & adipogenesis & $\begin{array}{l}\text { chemical - endogenous } \\
\text { mammalian }\end{array}$ & 49 & 0.040 \\
\hline Inhibited & Prolactin & early in adipogenesis & cytokine & 30 & $<0.001$ \\
\hline Activated & miR-124-3p & adipogenic & mature microRNA & 24 & 0.001 \\
\hline \multicolumn{6}{|c|}{ Oxidative stress } \\
\hline Inhibited & Genistein & PPARs, NRF, autophagy & chemical drug & 34 & $<0.001$ \\
\hline Inhibited & NFE2L2, =NRF2 & antioxidant defense & transcription regulator & 31 & 0.001 \\
\hline \multicolumn{6}{|c|}{ Inflammation and Immune defense } \\
\hline Inhibited & TNF- $\alpha$ & inflammation & cytokine & 49 & 0.023 \\
\hline Activated & $\begin{array}{l}\text { Sirolimus } \\
\text { (=Rapamycin) }\end{array}$ & $\begin{array}{l}\text { impairs T- \& B-cell } \\
\text { proliferation and } \\
\text { activation }\end{array}$ & chemical drug & 29 & 0.007 \\
\hline \multicolumn{6}{|c|}{ Anti-carcinogenic } \\
\hline Inhibited & Trichostatin A & $\begin{array}{l}\text { antibiotic, inhibits histone } \\
\text { deacetylase }\end{array}$ & chemical drug & 30 & 0.040 \\
\hline Inhibited & Gentamicin & $\begin{array}{l}\text { antibiotic, binds bacterial } \\
\text { ribosome }\end{array}$ & chemical drug & 24 & 0.002 \\
\hline Activated & 5-Fluorouracil & cytostaticum & chemical drug & 26 & $<0.001$ \\
\hline Activated & CD 437 & $\begin{array}{l}\text { adipogenesis, anti- } \\
\text { carcinogenic (by ER- } \\
\text { stress) }\end{array}$ & chemical drug & 25 & $<0.001$ \\
\hline
\end{tabular}

PPAR-a, Peroxisome proliferator-activated receptor a; NFE2L2, Nuclear factor (erythroid-derived 2)-like 2 (=NRF2); TNF-a, tumor necrosis factor alpha. $\mathrm{P}$, statistical significance of activation/inhibition of upstream regulator for post versus preenrichment.

\section{Supplemental Table S6.4: Primer Sequences for RT-qPCR}

\begin{tabular}{|lll|}
\hline Gene name & Forward Primer & Reverse Primer \\
\hline ATP6V1A & GAGATCCTGTACTTCGCACTGG & GGGGATGTAGATGCTTTGGGT \\
\hline ATP6V1H & CAGAAGTTCGTGCAAACAAAGTC & TCAGGGCTTCGTTTCATTTCAA \\
\hline CD68 & CCCTATGGACACCTCAGCTTT & GAAGGCACATTGTACTCCACC \\
\hline HSL (LIPE) & GCGGATCACACAGAACCTGG AC & AGCAGGCGGCTTACCCT CAC \\
\hline LAMP2 & GAAATGCCACTTGCCTTTATGC & AGGAAAAGCCAGGTCCGAAC \\
\hline PI4K2A & TTGTCCTTAACCAGGGCTATCT & GTACGGGGAACAATGTTGAGT \\
\hline UCP2 & GGAGGTGGTCGGAGATACCAA & ACAATGGCATTACGAGCAACAT \\
\hline Endogenous control & Forward Primer & Reverse Primer \\
\hline GAPDH & GAGTCAACGGATTTGGTCGT & TTGATTTTGGAGGGATCTCG \\
\hline
\end{tabular}

Primer sequences for SYBR-Green based RT-qPCR analyses. F, forward; R, reverse. 


\section{CHAPTER 7}

\section{General Discussion}

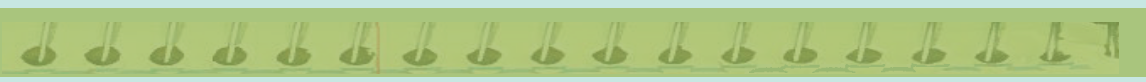

Ines Warnke, Joseph Schwager, Wim Saris, Ellen E. Blaak, Johan W.E. Jocken To be submitted 
During diet-induced weight gain adipose tissue (AT) expands due to increased fat storage, which may lead to alterations such as impaired lipid accumulation and angiogenesis, hypoxia, fibrotic tissue [1] and an imbalance in the secretion of pro- and anti-inflammatory proteins [2]. These changes can, at least in part, be reversed through weight loss, which affects AT metabolism, resulting in decreased adipocyte size, number of AT macrophages [3] and increased adipogenic capacity of pre-adipocytes [4], all accompanied by a less inflammatory secretion profile. Eventually weight loss can reduce the risk for diabetes [5] because it significantly enhances systemic insulin sensitivity [6]. However, metabolic impairments are more correlated with AT morphology and function than with body fat mass per se $[7,8]$. Which genetic, behavioural, and environmental interactions alter white AT morphology and function, including its ability to expand, is still debated. Nevertheless, the induced changes may lead to AT inflammation, lipid overflow, and ectopic fat storage [9], consequently impairing peripheral glucose disposal and systemic insulin sensitivity, and stimulating systemic low-grade inflammation [10]. These alterations are associated with the increase of the obesity-related diseases T2DM, CVD and NAFLD [11]. Consequently, an alleviation of subcutaneous AT (scAT) dysfunction by restoring its adipogenic potential, secretory profile, insulin sensitivity and lipid buffering capacity [11-13], or a redistribution of lipids into the beneficial femoral AT depots [14], favours metabolic health. Modifying AT (dys)-functions and inflammation with nutritional bio-actives offers a potential therapeutic option, alone or as add-on therapy to other weight loss strategies, to antagonize obesityrelated metabolic impairments. Therefore, the objective of this thesis was to investigate whether nutritional bio-actives (polyphenols, carotenoids and n-3 PUFAs) affect human white AT biology, hence contributing to improvements of metabolic profiles in humans.

\section{Favorable modulations of AT (dys)-functions and metabolic profiles}

Due to the increasing prevalence of obesity-related comorbidities and the limited success of promoting lifestyle changes, human intervention studies are conducted to investigate nutritional bio-actives as additional preventive or therapeutic strategy. These studies demonstrated that the supplementation with the polyphenols trans-resveratrol (Res) and epigallocatechin-3-gallate (EGCG; green tea extract (GTE)) decreased plasma glucose, insulin and lipid levels and ameliorated insulin sensitivity, blood pressure and markers of inflammation independent of changes in body-weight [15-18]. They may also improve AT function as shown for Res by a shift to smaller fat cells and modifications of AT lipolysis and inflammation [19, 20]. Human studies investigating the impact of combinations on the regulation of adipocyte metabolism and AT 
(dys-)functions are scarce and results for single constituents are often controversial. This calls for more nutritional studies testing combinations of bio-actives with distinct mechanism of action under controlled settings and for supportive in-vitro experiments with primary human adipocytes to elucidate mechanisms underlying the impact of bio-actives on AT function.

\section{Role of nutritional bio-actives in modifying the morphology and gene expression of adipocytes and $A T$}

The morphology of AT is a determinant for its functionality and accordingly, hypertrophic AT (enlarged adipocytes) is more insulin resistant and lipolytically active, and secretes more proinflammatory cytokines than hyperplastic AT (increased number of smaller adipocytes) $[7,21$, 22]. In-vitro adipocyte cultures can partly mirror AT metabolism and therefore we developed an automated fluorescent assay with murine $\mathrm{C} 3 \mathrm{H} 10 \mathrm{~T} 1 / 2$ adipocytes to measure effects of dietary constituents on the lipid accumulation in-vitro (Chapter 2). This high content assay is a novel robust method to quantify changes in cytosolic lipid droplet (LD) content during adipocyte differentiation, and was validated with nutritional substances that had been shown to affect murine adipogenesis. Compared to the commonly utilized Oil Red O staining procedure [23], the assay eliminates the tedious error-prone microscopically counting of LDs, and concomitantly determines size and intensity of the LDs. Additionally, we demonstrated that n-3 PUFAs and beta-carotene (dose-dependent reduction of LDs), but also (all-E)-lycopene (Lyc) and hydroxytyrosol significantly reduced lipid accumulation. Our findings are in line with effects described for EGCG [24], docosahexaenoic acid [25] and beta-carotene [26], while the inhibitory effects of Lyc and the lack of effects of Res, described as anti-adipogenic in murine cell models [27], were unexpected.

Studies with murine cell lines only give hints how and which bio-actives might influence the storage of LDs in human AT and several natural substances seem to act on different biological pathways of AT. Therefore, we conducted in-vitro studies with primary human white adipocytes: i) to confirm the previous murine data and ii) to test whether combinations of dietary bio-actives (with distinct mechanisms of action) are more efficacious regarding inhibition of lipid accumulation than individual constituents (Chapter 3 and 4). Human multipotent adipose-derived stem (hMADS) cells were isolated from subcutaneous white AT of age-matched lean and obese donors (Chapter 4). The effects observed with the murine adipocytes were only partly consistent with those of the human adipocytes, which might be indicative for species differences. However, we demonstrated for the first time that combining Lyc and/or eicosapentaenoic acid (EPA) with Res significantly reduced the lipid accumulation in in-vitro differentiated human adipocytes (Chapter 3). The combination Lyc/Res was also anti-lipogenic when using slightly modified 
differentiation conditions (less rosiglitazone $(5 \mu \mathrm{M})$, Chapter 4). However here, Res seemed to be the key driver of the altered features in differentiated hMADS cells, because it diminished LD accumulation and blunted basal and ISO-stimulated lipolysis to the same extent as the combination, whereas Lyc showed no effect. Hypertrophic insulin resistant AT displays alterations in adipogenic and lipolytic gene and protein expression [28, 29], hence we further investigated whether the bio-actives modified the transcriptional profile of adipocytes. Indeed, the effects on the LD quantity in in-vitro differentiated murine and human adipocytes could, in part, be related to changes in the balance between the adipogenic, lipogenic and lipolytic gene expression. The anti-adipogenic effects of Res have been attributed to the activation of AMP-activated protein kinase (AMPK) [30] and SIRT1 [31], which repress the regulators of adipogenesis, PPAR-gamma and C/EBP-alpha. Such a suppression was observed in our human adipocytes by Res and the combinations Lyc/Res and EPA/Res, which led to a subsequent down-regulation of the lipogenic markers GLUT-4, LPL, FAS and FABP-4. GLUT-4 protein levels in AT gauge adipose insulin sensitivity and a decreased GLUT-4 mRNA and protein content is described in insulin resistant overweight, pre-diabetic and T2DM subjects $[32,33]$. Therefore, a suppression of GLUT-4 and LPL mRNA by bio-actives is not beneficial when it translates into reduced protein levels and finally diminishes glucose uptake and TAGs/FFAs buffering capacity of adipocytes. Accordingly, the effects of bio-actives on protein expression, translocation, and phosphorylation status and on functional uptake need to be investigated in further evaluations. The same applies for the antilipolytic protein PLIN-1 and the lipases ATGL and HSL, which were in contrast to other studies $[34,35]$ significantly suppressed by Res and the combination Lyc/Res, resulting in a blunted lipolytic activity in hMADS cells (Chapter 4). This is in agreement with the concept that partial inhibition of adipocyte lipolysis (using pharmacological ATGL and HSL blockers) could prevent ectopic lipid storage and development of insulin resistance under high fat diet (HFD) conditions $[36,37]$.

Taken together, AT biology is species-specifically regulated, which hampers comparisons of findings in murine and human adipocytes. Furthermore, conflicting effects of bio-active substances in human cellular assays could be explained by the application of varying culture and differentiation conditions. These comprise different cell passages, seeding densities, concentrations of i) FCS ( $0 \%-10 \%)$ and cAMP elevating agents, ii) varying hormones and proteins (e.g., dexamethasone, cortisol, insulin, transferrin, and triiodothyronine (T3)), and iii) the use of varying PPAR-gamma ligands (thiazolidinediones (TZD)) at different stages during the adipocyte differentiation [27, 38-41]. Indeed, an alteration of the differentiation conditions, e.g. by reducing 
the level of the PPAR-gamma agonist rosiglitazone, dramatically changed the metabolic response of differentiating human pre-adipocytes (HPAd) to bio-actives e.g. Res, although the morphological features (LD quantity) of the 14 days differentiated CTRL cells were comparable (Chapter 3 and 4). As the differentiation of human adipocytes is often induced via low rosiglitazone concentrations during the first 3 days, we hypothesize that our hMADS cell differentiation procedure (Chapter 4) emphasized effects of the bio-actives that are more comparable with formerly reported results e.g., for Res [42, 43]. Interestingly, other groups use rosiglitazone together with T3 for the formation of beige/brown-adipocytes [40]. This raises the question, whether we study beige or white adipocyte metabolism and function in-vitro.

\section{Combining nutritional bio-actives: in-vitro versus in-vivo effects on AT}

Nutritional bio-actives that decrease the volume of existing and increase the number of adipocytes and thus induce hyperplastic WAT expansion in-vivo, are considered major preventive and therapeutic measures against metabolic complications of obesity [33]. Yet, we showed in a randomized, double-blind, placebo (PLA) controlled study that a 12-week EGCG/Res supplementation did not change the abdominal scAT morphology in overweight-obese subjects (Chapter 6). Due to the unilocular LDs in adipocytes in-vivo, we determined the cells size and not the LD number (in-vitro) as indicator of lipid content. The unaltered adipocyte morphology is in contrast with the reduced subcutaneous adipocyte size shown by Konings et al. [19] after a 4-week treatment with Res only $(150 \mathrm{mg} / \mathrm{d})$. However, it is in line with the unchanged body fat mass reported in Chapter 5 and by other groups that supplemented Res [44, 45] or EGCG [46, 47] over 12 weeks. Ahmed et al. illustrated that the combination EGCG/Res additively inhibited in-vitro lipid accumulation in HPAd and 3T3-L1 adipocytes (15 $\mu \mathrm{M}$ or $30 \mu \mathrm{M}$ each, respectively), which were differentiated with the same adipogenic cocktail (no TZD). The individual bio-actives displayed no inhibitory effects on adipogenic differentiation [48]. Together these data indicate that translation from in-vitro to the in-vivo physiology is limited, also due to the fact that bio-actives may affect different metabolic processes: in-vivo TAGs, stored in adipocytes, are generated from FAs which are derived from chylomicrons and VLDL via extracellular lipolysis, whereas FAs / TAGs in in-vitro differentiated adipocytes are mainly synthesized from glucose via de-novo lipogenesis. Furthermore, a reduced fat storage capacity of adipocytes in-vitro is not necessarily favorable in metabolically compromised subjects because without compensatory mechanisms like increased recruitment of new adipocytes or fat oxidation, the excess of dietary fat is likely to be stored ectopically [49]. Yet, a reduced lipid content in hypertrophic AT could decrease features of AT dysfunction like hypoxia and fibrosis [50]. 
Although EGCG/Res supplementation did not influence AT morphology, the gene chip data indicated that this combination induced significant changes in the ScAT gene expression profile depicted by a suppression of gene sets related to adipocyte differentiation, inflammation, and the immune system. More specific, a simultaneous down-regulation of gene expression markers of adipogenesis, autophagy and apoptosis implies that EGCG/Res reduced adipocyte turnover, as discussed in Chapter 6. A low adipocyte turnover may increase the risk for hypertrophic fat cells and subsequently reduces insulin sensitivity [7]. Yet, we did not observe any changes in subcutaneous adipocyte size after 12 weeks. Nevertheless, during weight gain, a reduced adipocyte turnover could impair metabolic functions and health, which requires further studies. As AT of obese subjects can consist of over $50 \%$ of immune cells, the 'AT' transcriptional profile reflects an overall gene expression of all cells within the tissue [51]. Thus, the observed down-regulation of inflammatory signaling pathways after EGCG/Res supplementation suggests that AT inflammation is attenuated. This could on the one hand ameliorate AT function and preclude systemic low-grade inflammation [52], but on the other hand might affect healthy AT expansion as this is dependent on well-functioning inflammatory processes [53]. However, microarray results from other Res intervention studies reported opposite [15] or no effects [45], indicating that the combination might exhibit different effects than the single polyphenols per se, and gives rise to interpret these data circumspectly in the absence of the corresponding control groups.

\section{Improvement of adipose insulin sensitivity, and lipolytic and mitochondrial capacity}

Dysfunctional and insulin resistant adipocytes, exhibiting low GLUT-4 levels and a high adipo/cytokine (e.g. RBP-4) secretion, mainly contribute to a decline in whole-body insulin sensitivity $[33,54]$. Hence, modifying AT functions by decreasing the size of large adipocytes with bioactives, would create smaller insulin sensitive adipocytes. Therefore, we measured the AT-specific lipolytic activity, insulin sensitivity and mitochondrial capacity after 12-week EGCG/Res supplementation (Chapter 5 and 6). Unexpectedly, a down-regulation of genes related to mitochondrial and energy metabolism following EGCG/Res did not translate into a decreased expression of oxidative phosphorylation (OxPhos) proteins in AT. In line, AT lipolysis (measured by in situ microdialysis) and AT insulin sensitivity (measured as \% suppression of plasma FFA during a hyperinsulinemic-euglycemic clamp) were not influenced by the intervention, although a tendency of visceral AT (VAT) mass reduction (measured by DEXA), was noted. In comparison, a 30-day treatment with Res led to a slightly reduced postprandial AT lipolysis, i.e. an indication for an improved insulin sensitivity [15]. In accordance with this study differentiated hMADS cells in Chapter 4 showed a blunted Res-mediated lipolysis. However, these findings contradict the 
suggestion that Res enhances stimulated lipolysis in adipocytes [55], what may lead to ectopic lipid storage after long-term supplementation of bio-actives in humans, if no compensatory increase in fat oxidation is induced in these non-adipose tissues. Interestingly, AMPK signaling, the main switch regarding fuel metabolism and energy homeostasis [56], and uncoupling proteins were also downregulated in AT after 12-weeks EGCG/Res supplementation. This is contrary to the growing evidence from in-vitro and rodent studies, that nutritional bio-actives can activate AMPK [57, 58] and stimulate browning of WAT [59], thereby mediating their anti-obesity potential [60].

\section{Role of nutritional bio-actives in modulating AT inflammation and secretory activity}

In AT of obese humans more than $90 \%$ of the adipo-/cytokines are secreted by non-adipose cells [61] and these molecules modify pro-and anti-inflammatory responses in AT itself and other tissues, e.g. liver and muscles [1]. Because large adipocytes are characterized by a shift towards a pro-inflammatory AT profile [21], a reduction in lipid content should attenuate AT inflammation and consequently metabolic dysfunction [62]. As shown in Chapter 3 and 4, the morphological changes induced by Res, EPA and the combinations in in-vitro differentiating human adipocytes were accompanied by a reduced secretion of the pro-inflammatory cytokines IL-6, MCP-1 and HGF. Numerous in-vitro and in-vivo studies illustrated similar effects of Lyc, Res, and EPA, as they reduced inflammatory cytokines (IL-6, MCP-1, PAI-1 and TNF-alpha) and lowered systemic and HDL-associated inflammation in human adipocytes [42, 63-65], obese or diabetic rodents [6669], and humans [70-73]. These studies also underline that the health promoting effects of EPA are mostly attributed to its activation of the G-protein coupled receptor (GPR) 120 [74] and its anti-inflammatory and pro-resolving lipid mediators [75, 76]. Furthermore, these studies corroborate that the anti-inflammatory effects of nutritional bio-actives are based on the inhibition of NF-kappaB [77-80], which regulates adipocyte-specific cytokine secretion [81]. In Chapter 3 and 4, we demonstrated that Lyc, Res, and EPA suppressed the secretion of IL-6 and MCP-1 without prior stimulation of inflammatory pathways. This could be translated in-vivo into a reduced attraction of macrophages into AT [82] and consequently prevent inflammation and insulin resistance [83]. Astonishingly, the lipophile Lyc, showed only moderate anti-inflammatory effects. In comparison, Res and the combination Lyc/Res were much more effective regarding the suppression of pro-inflammatory cytokine release and showed comparable effects. This reemphasises that Res seems to be the key driver for the observed anti-inflammatory shift under noninflamed conditions, whereas Lyc may attenuate inflammation in induced models [63, 84]. Surprisingly, Res and the combinations significantly reduced adiponectin secretion in-vitro. This 
is in contrast to recent findings with murine and human adipocytes $[42,85,86]$ and human AT explants [20], where Res and EPA increased adiponectin secretion or prevented its suppression by TNF-alpha. Alternatively, the chronic presence of bio-actives may have indirectly reduced adiponectin production by inhibiting differentiation into human fat cells. In-vivo we did not observe any impact of EGCG/Res supplementation on plasma IL-6, IL-8, TNF-alpha, adiponectin, and leptin levels (Chapter 5). This opposes our in-vitro results and other animal and human studies testing Res [87, 88] and EGCG [17, 89]. In summary, both blocking pro-inflammatory and/or increasing anti-inflammatory proteins in obese AT might ameliorate metabolic profiles and diminish obesity-related metabolic diseases [90], although it was recently proposed that ATinflammation is essential for healthy WAT expansion [53]. As discussed above and reviewed recently [60,91], nutritional bio-actives bare a great potential in contributing to these improvements and warrant further investigations. Obviously, nutritional bio-actives can not only affect AT functions such as adipo-/cytokine secretion and thus indirectly influence other tissues, but also directly act on the cross-talk between organs and modify their functions and whole-body metabolic parameters.

\section{Impact of supplementation with nutritional bio-actives on insulin sensitivity and metabolic health}

Res and EGCG might affect metabolic profiles and insulin sensitivity, as seen in diet-induced obese rodents and human intervention studies, possibly through modifications of energy metabolism and substrate oxidation (mitochondrial function) in peripheral organs. These effects are mediated via the activation of the AMPK pathway (by both) and SIRT1 / PGC-1alpha stimulation (by Res: $[15,92,93]$ ) or catechol-O-methyl-transferase inhibition [by EGCG: $[17,94-$ 96]). Timmers et al. reported that Res increased insulin sensitivity in obese men, but decreased fasting and postprandial energy expenditure (EE) [15], which could lead to weight gain during long-term supplementation (>30 days). They proposed that EGCG's EE increasing effects [97] could counteract Res's "side symptom". Indeed, a 3-day EGCG/Res intervention in overweight subjects showed increased resting EE and metabolic flexibility [98]. Based on these short-term metabolic effects it was hypothesized that the combination EGCG/Res might also improve insulin sensitivity and metabolic profiles in long(er)-term studies. Therefore, we performed a randomized, double-blind parallel study to investigate whether i) the supplementation with EGCG/Res can increase insulin sensitivity and ii) the positive short-term metabolic effects could be maintained for 12 weeks in non-diabetic overweight-obese subjects (Chapter 5). Forty-two male and female 
overweight-obese subjects were examined before and after a 12-week intervention with EGCG/Res ( $\mathrm{n}=20,282$ / $80 \mathrm{mg} / \mathrm{d}$, respectively) or PLA ( $\mathrm{n}=22)$. The primary outcome was tissuespecific insulin sensitivity (peripheral, hepatic and AT insulin sensitivity as assessed by a hyperinsulinemic-euglycemic clamp). Secondary outcomes were body composition, skeletal muscle (SM) oxidative capacity, fat oxidation, lipolysis (via microdialysis) and AT and SM gene expression profiles.

\section{Effects on tissue-specific insulin sensitivity}

Improvements in whole-body lipid metabolism as observed in Chapter $\mathbf{5}$ are suggested to increase insulin sensitivity $[99,100]$ and improve obesity-associated metabolic risk factors (CVD, [101]). However, the observed improvement in whole-body lipid metabolism (discussed below) and the tendency in VAT mass reduction after EGCG/Res did not translate into improved HOMA-IR values or clamp-derived tissue-specific insulin sensitivity (peripheral, liver, AT). The unaffected insulin sensitivity is reflected by an unchanged expression of insulin signaling-related genes in AT and SM, an unmodified skeletal muscle lipid content, and no changes in markers of systemic inflammation. In agreement with our observation (Chapter 5) are two studies that reported unchanged insulin sensitivity after 4- or 12-week Res supplementation [44, 45]. Nevertheless, the slight increase $(\sim 11 \%)$ in SM mitochondrial capacity with EGCG/Res (Chapter 5), may be insufficient to impact insulin sensitivity after 12 weeks, but in the long run it could prevent insulin resistance by delaying a decline in mitochondrial function. In addition, the maintenance of adequate whole-body fat oxidation with EGCG/Res during fasting and in postprandial conditions (Chapter 5) may minimize mitochondrial overload and hence the built-up of harmful lipids and reactive oxygen species (ROS). Because EGCG and Res seem to modulate fasting glucose and insulin concentrations (HOMA-IR), they are regarded insulin sensitizing bio-actives [102, 103]. However, pronounced beneficial effects of Res on insulin sensitivity were previously mainly observed in populations that had already, in contrast to our population (normoglycemic and mildly insulin resistant), metabolic impairments (e.g. T2DM and NAFLD) [103, 104]. So, it appears likely that an improvement in insulin sensitivity by Res is dependent on the donor characteristics, in particular the severity of the metabolic impairment. A study that investigated a low-caloric diet with or without EGCG supplementation, using a comparable dose (300 mg/d, 12 weeks) as in our study, showed no additional effect of EGCG on measured metabolic parameters, including HOMA-IR, and liver function in obese women [105]. Furthermore, a 4-week Res intake (150 $\mathrm{mg} / \mathrm{d}$ ) did not influence markers of (cardio-)metabolic risk (BMI, HOMA-IR) and inflammation (IL-6, TNF-alpha) in overweight-obese subjects [106]. Although, a one-year supplementation with low doses of Res ( 8 and $16 \mathrm{mg}$ ) showed anti-inflammatory effects in patients with CVD risk using 
statins [107], emphasizing the dosage question (see below). Contrary to animal studies, the more recent systematic review by Christenson et al. concluded that there is insufficient evidence for Res to be used as anti-obesity supplement in humans [104], as the majority of reviewed studies reported no effects of Res on their primary outcomes of interest (body mass, fat mass and central obesity) in overweight-obese subjects. This conclusion and published reports of health-improving effects in mainly metabolically compromised subjects $[102,103]$ agree with our results that showed no effect of the combination on body weight and composition, and insulin sensitivity in obese but otherwise metabolically healthy subjects. Besides its putative effects regarding glycemic control and insulin sensitivity [108], Res and also EGCG may be potent bio-actives to reduce inflammation and oxidative stress, consequently contributing to metabolic health or being used in chemoprevention [109]. Thus, it remains to be investigated whether the combined EGCG/Res supplementation could exhibit effects on insulin sensitivity through its effects on lipid metabolism in obese with severe insulin-resistance or diabetic subjects.

\section{Effects on lipid metabolism - mitochondrial function, ectopic lipid storage (skeletal muscle) and fat oxidation}

Although 12-week EGCG/Res supplementation did not alter body weight and EE (discussed further below), it induced increased mitochondrial capacity of SM and attenuated systemic parameters, besides the effects on adipose function (Chapters 3, 4, and 6). Both polyphenols activated AMPK and PGC-1alpha, the master stimulator of mitochondrial biogenesis [92, 95], probably adding to the increased mitochondrial capacity observed after 12 weeks EGCG/Res intake (Chapter 5). This is in accordance with the increased oxidative metabolism found in overweight-obese men after Res (30 days) and EGCG/Res (3 days) supplementation [15, 98]. The ex-vivo increased SM oxidative capacity ( $11 \%$, measured as oxygen consumption rate) described in chapter 5 was comparable to the increase in respiratory capacity $(\sim 10 \%)$ after 4 -week Res supplementation [15], although only in our study (Chapter 5) a concomitant increase in the expression of OxPhos proteins was observed. This indicates that longer and combined supplementation does not further increase mitochondrial function per se but might rather induce mitochondrial biogenesis. Although this conclusion needs to be confirmed by measuring the mtDNA copy number. In line with these findings, the gene expression of mitochondrial pathways was also upregulated after 12 weeks. However, the gene expression profile in skeletal muscle differed from that in AT (Chapter 6), pointing towards a tissue-specific effect of combined polyphenol supplementation on the transcriptional level. ROS formed during mitochondrial respiration modulate many cellular processes. A temporary increase in ROS by HFD or exercise promoted mitochondrial biogenesis in SM $[110,111]$, whereas a persistent high ROS production, 
as seen in SM and AT of obese insulin resistant subjects [112-114], impaired mitochondrial function and activated inflammatory pathways, presumably contributing to insulin resistance [115]. As polyphenols can enhance ROS scavenging [116, 117], this may also account for the increase of mitochondrial capacity in our study (Chapter 5). It is still debated if mitochondrial dysfunction accelerates insulin resistance or if insulin resistance contributes to mitochondrial dysfunction (for review see [118]). Nevertheless, supplementing nutritional bio-actives would provide a strategy to interrupt this mutual interference as it may improve either mitochondrial function (our study) or insulin sensitivity [108]. Both are impaired in obese and diabetic subjects and promoted by physical inactivity and overeating $[119,120]$.

Concomitantly, we observed a slight increase in whole-body fat oxidation in the EGCG/Res compared to a slight decrease in the PLA group, while EE remained unchanged in both groups (Chapter 5). These findings may be explained by the addition of EGCG and the duration of the intervention, because Timmers et al. observed no increase in fat oxidation after 30-day Res supplementation in healthy and diabetic obese men $[15,121]$. Indeed, it has been shown that EGCG or GTE can stimulate fat oxidation and suppress the increase of plasma TAG levels after longer supplementation periods [47, 122-124]. This effect on fat oxidation could also be ascribed to the increased mitochondrial capacity. Indeed, such an increased oxidative capacity in skeletal muscle, after 12-week EGCG/Res, could be interpreted as "endurance exercise training" mimicking effect, with a greater reliance on fat oxidation and hence, reduced carbohydrate utilization, glycogen depletion [125], and lactate production. This is in line with recent findings for EGCG [126].

Lipid-spill over from dysfunctional scAT promotes ectopic lipid storage with its consequent lipotoxicity [49]. After 12-week EGCG/Res supplementation scAT mass and morphology were unchanged, although the VAT mass tended to be decreased, and the lipid content and composition in SM was not altered (Chapter $\mathbf{5}$ and $\mathbf{6}$ ). This may reduce the risk of developing cardiometabolic diseases, since high visceral and skeletal lipid accumulation (untrained subjects [127]) are associated with cardiovascular, T2DM and metabolic inflammation risk [128-130]. Supplementation of EGCG or Res in rodents has shown to reduce VAT [66, 131, 132] and to increase hepatic fat oxidation [133, 134]. Based on this, one could speculate that the decrease in VAT may have improved hepatic function [129] and contributed to the decreased intrahepatic lipid content in obese men after Res intake [15]. As we neither measured hepatic lipid content nor substrate oxidation, and hepatic insulin sensitivity was unchanged, the liver's contribution to the preserved whole-body fat oxidation by the polyphenols needs to be investigated in future research 
by combining tracer methodology with advanced metabolic imaging techniques (e.g. MRI / MRS). Additionally, the combination of EGCG/Res did not impact local and systemic lipolytic markers such as interstitial glycerol or plasma FFA levels. This implies that the metabolically beneficial outcomes seem not mediated by altered lipolysis in SM and AT as reported in man and mice after Res intake $[15,135]$. Taken together, we observed an overall higher fat oxidation during fasting and postprandially and a blunted increase of postprandial plasma TAG after a high fat mixed meal (HFMM), which may impede fat accumulation in adipose and non-adipose tissues and could improve the disturbed lipid-handling observed in skeletal muscle of obese insulin resistant-diabetic subjects $[136,137]$. Therefore, combined EGCG/Res intake might potentially contribute to the prevention of ectopic lipid storage and associated insulin resistance when supplemented for longer periods.

\section{Effects on energy expenditure, body weight and composition}

In our 12-week intervention study (Chapter 5), combining Res with EGCG did indeed prevent a previously observed Res-induced (after 30 days) decrease of whole-body EE [15]. However, the increased resting EE observed after 3-days treatment with EGCG/Res [98] and the insulin sensitizing effect reported for Res alone [15] could not be confirmed in our combined long-term supplementation study. In agreement with the unaffected EE are the unchanged food intake, body weight and composition, although there was a trend towards a decreased VAT mass $(\sim 11 \%)$ after 12 weeks EGCG/Res intake. Nonetheless, EGCG/Res supplementation significantly preserved whole-body fat oxidation (relative increase as compared to PLA), increased mitochondrial capacity in SM, and prevented a postprandial increase of TAG plasma levels compared with PLA. These findings are consistent with a rodent study which described that chronic upregulation of whole-body fatty acid oxidation (by pharmacological or genetic means) did not alter EE and total body fat mass [138]. Again, the translation of results from rodents to humans is inadequate, because Res or EGCG prevented body weight gain and visceral fat accumulation in rodent models were obesity was induced using a HFD $[66,131]$, whereas in human intervention studies already obese subjects were supplemented. So far, no consistent effects on body weight and composition have been reported after supplementation of humans with polyphenols (reviewed in [104, 139]). This underlines that polyphenols might have species-specific modes of actions that furthermore depend on the bioavailability and dosage.

\section{Bioavailability and dosage of nutritional bio-actives}

Overall, bioavailability of nutritional bio-actives including polyphenols is poor $[140,141]$ and makes the extrapolation of in-vitro data to in-vivo models difficult. Nevertheless, the 12 -week 
EGCG/Res supplementation (Chapter 5 and 6) increased the plasma levels of EGCG, Res and dihydro-Res significantly. The applied doses of EGCG (200-800 mg/d [142]) and Res (75-1500 $\mathrm{mg} / \mathrm{d}[44,45])$ in this and other intervention studies are considered as safe and well tolerated. Interestingly, a study in healthy subjects showed that catechin metabolites from orally supplemented GTE were incorporated into the skin after a 12-week treatment and protected it from UV-induced stress [143]. This shows that despite their low bioavailability, polyphenols and derived metabolites accumulated in distinct tissues where they exert their anti-inflammatory / antioxidant effects. An attempt to increase e.g., the bioavailability of Res is the co-administration with other polyphenols, which competitively inhibit metabolizing enzymes and thus delay the elimination of Res from the circulation [144]. This could explain why we measured higher plasma levels of Res (274 ng/ml, chap. 5 vs $183 \mathrm{ng} / \mathrm{ml}$ [15]) when supplementing $80 \mathrm{mg} / \mathrm{d}$ Res together with $282 \mathrm{mg} / \mathrm{d}$ EGCG compared to $150 \mathrm{mg} / \mathrm{d}$ Res alone as used by Timmers et al. [15]. The poor bioavailability of bio-actives from vegetable matrices can be enhanced when e.g. tomato products are processed and co-ingested with lipids $[145,146]$. Like for EPA, which is, regardless of its formulation, orally ample bioavailable [147], also Lyc plasma levels reach a plateau in healthy adults after a 4-week intake [146]. It was also suggested that requirements of fat-soluble carotenoids may be dependent on body composition, among other factors, as they are stored in fatty tissues including the AT and liver [148]. On the contrary, for certain bio-actives e.g. from tea extracts, a poor intestinal absorption is favorable as these extracts inhibit glucosidases and lipases and consequently may reduce absorption of macronutrients and hence energy uptake [149, 150]. Furthermore, the dietary composition or medication can impact the effectiveness and absorption of nutritional bio-actives. A study in mice demonstrated more pronounced effects of Res on lipogenesis and adipocyte size with a high protein diet compared to a HFD [151]. On the other hand, in humans the oral delivery of EGCG is highest when taken with water and under fasting conditions [152]. This shows that standardizing ingestion procedures could minimize the impact of individual diets. Finally, the biotransformation of polyphenols by gut microbiota and the modulation of gut microbiota by polyphenols can affect the bioactivity and bioavailability of polyphenols [153].

Besides bioavailability also the dosage of bio-actives is vital for their effectiveness. Because the optimal doses and the interactions for Res and EGCG supplements in humans are not known yet, we used lower doses for the combined polyphenol supplementation over 12 weeks. Interestingly, high doses of Res were either found not effective $(1500 \mathrm{mg} / \mathrm{d})$ [44] or significantly promoting $(3000 \mathrm{mg} / \mathrm{d})$ [154] with respect to metabolic parameters like insulin sensitivity and EE, 
respectively, whereas in both studies no change in body weight was reported after 12 weeks. EGCG presents a comparable picture: at higher doses $(\sim 600 \mathrm{mg} / \mathrm{d})$ it stimulated fat oxidation and 24-h EE [97] and exerted no effects in obese men [155], but at a low dose it increased the fat oxidation. However, also lower doses $(282 \mathrm{mg} / \mathrm{d})$ proved to be ineffective regarding EE and fat oxidation in obese subjects [126]. Along the lines, the more is not always the merrier, other intervention studies supplementing with 150-200 mg/d Res, reported an improved insulin sensitivity [15] and EE [98]. This is contrary to our unchanged EE and insulin sensitivity after EGCG/Res intake (Chapter 5). Strikingly, high doses of decaffeinated GTE (EGCG 857 mg/d, Asians) [18] and normal GTE (>540 mg EGCG + 360 mg caffeine/d) [46] showed no significant effects on body weight, composition, and other metabolic parameter (hormones, REE, RQ, lipid absorption) after 12 weeks. In fact, high doses of antioxidants may also induce unfavorable effects as shown by Ristow et al. who described that high doses of antioxidants (vitamin C (10x RDA) and $\mathrm{E}$ (20x RDA), 4 weeks) blunted the health-promoting effects of exercise e.g. increased insulin sensitivity, by exaggerated ROS scavenging [156]. Similar bimodal effects are described for n-3 PUFAs, with a significant reduction in markers of oxidative stress like malondialdehyde at lower concentrations, but an increase at high ones [157].

\section{Conclusions and Future Directions}

The human in-vitro (Chapter 3 and 4) and in-vivo studies (Chapter 5 and 6) described in this thesis contribute to the knowledge of how nutritional bio-actives can affect AT function, insulin sensitivity, and metabolic profiles and health. The effects of combined bio-actives (Lyc/Res) treatment on adipocyte features in-vitro did not translate into changes in body composition and improved tissue-specific insulin sensitivity and lipolysis in-vivo, although an elevated skeletal muscle mitochondrial capacity and increased whole-body fat oxidation was observed in overweight-obese adults after 12-week supplementation (EGCG/Res). Hence, it is important that future research considers the outcomes and limitations of this thesis (summarized below).

1. Our adipose tissue-related data (Chapter 2, 3, 4 and 6) support the concept that applying nutritional bio-actives is an effective strategy to correct metabolic impairments related to AT dysfunction [60, 158]. Combining multifunctional molecules that target several signaling pathways $[19,159,160]$ augments the probability of magnified health benefits at lower doses compared to the single dietary constituents [78]. Therefore, combining Res with other bio-actives could be advantageous for shifting the release of adipokines towards a less pro-inflammatory 
profile and for improving adipocyte functions (Chapter 3), consequently reducing/preventing chronic 'low-grade inflammation' and metabolic complications accompanying obesity [161, 162]. However, defining the precise molecular effects of differential doses of bio-actives [163] on AT biology requires further attention.

2. Primary human cells from well characterized donors represent subjects with distinct individual responses; this better mirrors the in-vivo situation than the use of other non-human adipocyte cell lines. However, all current cell models operate at distinct un-physiological conditions e.g. high glucose levels and aqueous medium. These conditions do not facilitate the creation of a proper ECM and the interaction with it or with neighboring cells [164], which may modify the differentiation of cells, activity of enzymes, secretion of cytokines $[165,166]$, and presumably the effects of test substances. Therefore, adaptations of culturing conditions e.g., via macromolecular crowding [40, 166, 167], co-cultures and 3-D scaffolding [164] would mimic more closely the microenvironments found in adipose tissue. In short, standardization of in-vitro differentiation assays for primary human white adipocytes as suggested by Lee et al. [39], including the use of macromolecular crowding and more sophisticated 3-D co-cultures, is urgently needed to reshape the "in-vitro landscape" for studying (synergistic) effects of nutritional bio-actives.

3. The in-vitro findings of this thesis suggest that the effects of nutritional bio-actives, like the polyphenol Res, on AT function might be largely affected by the applied differentiation protocol (Chapter 3 and 4), but could also be dependent on intrinsic donor characteristics, which needs to be investigated in more detail in future research. Therefore, a stringent harmonization of culture conditions is essential to corroborate the observed biological effects on adipocyte features and to elucidate the suppression of adiponectin secretion and lipolysis by Res and the combinations.

4. There is increasing evidence from numerous human studies that suggest beneficial effects of nutritional bio-actives on metabolic diseases. Yet, the optimal doses cannot be determined due to the variable designs of studies. As indicated in this thesis, there are several factors like the duration of the intervention and the addition of EGCG that may explain different and inconsistent findings. Besides the small sample size (Chapter 6), the lack of the additional adequate control groups is another limitation of the in-vivo studies. Thus, there is an urgent need to further standardize nutritional intervention studies to minimize confounding factors. The metabolic phenotype including habitual dietary intake and physical activity, BMI, and the sex-dependent body fat distribution $[14,168]$ (merging of overweight and obese men and women, this thesis), the dose, the supplement nature, and the duration of supplementation are factors that may influence 
intervention effects. Ideally, a cross-over study as designed and performed in other dietary intervention trials $[15,98]$ could reduce the influence of inter-individual variation as each participant is acting as their own control. Yet, this design is limited in study duration.

5. The inclusion of women in our study may have mitigated the effects of the polyphenols because women appear less responsive in this type of nutritional interventions [47, 98, 169], probably due to their general healthier status at baseline. Additionally, our small mixed population limits the comparability with data generated in studies enrolling only men or women [15, 45]. Therefore, nutritional research studies should be adequately powered, designed, and analyzed to identify sexrelated differences in responses. Preferably, assessments in pre-menopausal women should be aligned with their menstrual cycle. This and considering different metabolic phenotypes will create the urgently needed data on intervention responses in males, females, and metabolic subgroups to define responders and non-responders for prevention and add-on therapies [107, 121].

6. The inconsistency in results reported for human studies supplementing dietary bio-actives suggests that a more detailed characterization of the cohorts is crucial to better demarcate subgroups according to their specific metabolic risk ("healthy" versus metabolically impaired overweight-obese subjects [170]). So, future studies could be improved by also recording the intake of i) artificial sweeteners that are recommended by diabetes and heart health associations [171], and ii) micronutrients as their intakes are either too high or too low in the affluent world [172]. Intriguingly, the glycemic response to a given food is variable between individuals [173] and seems dependent on the sensitivity of the gut microbiota to sweeteners [174]. Hence, interindividual glycemic responses and personal micronutrient inadequacies might influence the effectiveness of bio-actives in relation to insulin sensitivity and should therefore be considered for stratifying the study population further. In line, the gut microbiota composition, which depends on the diet [175], affects the plasma metabolome and associates with insulin resistance [176] and thus might also modify effects of dietary supplements. Eventually, the application of -omics technologies could help to assess the genetic background of subgroups and to identify biomarkers e.g. metabolites of bio-actives that indicate the success of an intervention.

7. As addressed by the FATe project [177] and many others, answering the outstanding questions how AT expandability is regulated and which other factors besides cell size induce AT dysfunction, would increase the knowledge of AT biology, and promote a more efficacious use of medications and dietary supplements. Moreover, future in-vitro or dietary intervention studies applying combinations of bio-actives should rather investigate mixtures of different classes of 
constituents with more diverse activities [60] in comparison to single constituents and PLA. betaCarotene for example affects different molecular targets than Res and it was shown that carotenoids are promising regarding weight loss in overweight children [178]. In accordance, the supplementation of a juice and vegetable concentrate reduced systemic inflammation, improved TAG plasma profiles and insulin sensitivity in overweight boys and adults $[159,179]$.

To conclude, despite the promotion of balanced diets and enough physical activity obese people nowadays outnumber underweight people not only in the western-(ized) world, but globally [180]. Therefore, it is crucial to invest in additional nutritional strategies to support weight loss, prevent weight gain or at least improve AT function and metabolic health. The results of this thesis indicate that combinations of nutritional bio-actives can positively influence adipocyte functions in-vitro, illustrated by an anti-inflammatory secretion profile and diminished lipid storage and lipolytic activity, and prevent in-vivo a decrease of whole-body fat oxidation besides a stimulation of oxidative capacity in skeletal muscle, favoring weight maintenance. It seems that such combinations can counteract the effects caused by an energy-dense Western diet [172], which contains high amounts of fat and added sugar (fructose [181]). The observed prevention of metabolic deterioration under "challenging" conditions (HFMM, this thesis) seems in agreement with effects of grape polyphenols observed in rats and humans, that were challenged with a highfructose diet $[182,183]$. This, together with the growing evidence from animal and human studies, suggests that dietary supplements could antagonize effects of the obesogenic "cafeteria" diet. To this end, applying combinations of nutrients that influence different pathways of obesityassociated metabolic diseases might exceed the favorable effects of individual bio-actives and lead to additive or synergistic effects. So, supplementing bio-active mixtures represent a more integrated approach additive to life-style adaptations [184], including increased physical activity. Overall, bio-actives could enhance the outcome of behavioral/dietary changes, with respect to improved metabolic health, body weight control, and exercise performance as was demonstrated for single polyphenol supplementation $[123,124,184]$. Nevertheless, such a comprehensive strategy can only be effective together with an altered supply of food products. This needs to be addressed by public health guidelines, and could include limiting and taxing or not subsidizing of unhealthy foods [185]. 


\section{References}

1. Bastard J-P, Fève B: Physiology and Physiopathology of adipose tissue: Springer Science \& Business Media; 2012.

2. Alligier M, Meugnier E, Debard C, Lambert-Porcheron S, Chanseaume E, Sothier M, Loizon E, Hssain AA, Brozek J, Scoazec JY et al: Subcutaneous adipose tissue remodeling during the initial phase of weight gain induced by overfeeding in humans. The Journal of clinical endocrinology and metabolism 2012, 97(2):E183-192.

3. Kovacikova M, Sengenes C, Kovacova Z, Siklova-Vitkova M, Klimcakova E, Polak J, Rossmeislova L, Bajzova M, Hejnova J, Hnevkovska Z et al: Dietary intervention-induced weight loss decreases macrophage content in adipose tissue of obese women. International journal of obesity 2011, 35(1):91-98.

4. Rossmeislová L, Mališová L, Kračmerová J, Tencerová M, Kováčová Z, Koc M, Šiklová-Vítková M, Viquerie N, Langin D, Štich V: Weight Loss Improves the Adipogenic Capacity of Human Preadipocytes and Modulates Their Secretory Profile. Diabetes 2013, 62(6):1990-1995.

5. Hamman RF, Wing RR, Edelstein SL, Lachin JM, Bray GA, Delahanty L, Hoskin M, Kriska AM, Mayer-Davis EJ, Pi-Sunyer X et al: Effect of weight loss with lifestyle intervention on risk of diabetes. Diabetes care 2006, 29(9):2102-2107.

6. Konings E, Corpeleijn E, Bouwman FG, Mariman EC, Blaak EE: Expression of genes involved in lipid metabolism in men with impaired glucose tolerance: impact of insulin stimulation and weight loss. Journal of nutrigenetics and nutrigenomics 2010, 3(1):9-17.

7. Arner E, Westermark PO, Spalding KL, Britton T, Ryden M, Frisen J, Bernard S, Arner P: Adipocyte turnover: relevance to human adipose tissue morphology. Diabetes 2010, 59(1):105109.

8. Hammarstedt A, Graham TE, Kahn BB: Adipose tissue dysregulation and reduced insulin sensitivity in non-obese individuals with enlarged abdominal adipose cells. Diabetology \& metabolic syndrome 2012, 4:42-42.

9. Suganami T, Tanaka M, Ogawa Y: Adipose tissue inflammation and ectopic lipid accumulation. Endocrine journal 2012, 59(10):849-857.

10. Leon-Pedroza JI, Gonzalez-Tapia LA, del Olmo-Gil E, Castellanos-Rodriguez D, Escobedo G, Gonzalez-Chavez A: [Low-grade systemic inflammation and the development of metabolic diseases: from the molecular evidence to the clinical practice]. Cirugia y cirujanos 2015, 83(6):543551.

11. Blüher M: Adipose tissue dysfunction contributes to obesity related metabolic diseases. Best Practice \& Research Clinical Endocrinology \& Metabolism 2013, 27(2):163-177.

12. Arner P, Bernard S, Salehpour M, Possnert G, Liebl J, Steier P, Buchholz BA, Eriksson M, Arner $\mathrm{E}$, Hauner $\mathrm{H}$ et al: Dynamics of human adipose lipid turnover in health and metabolic disease. Nature 2011, 478(7367):110-113.

13. Frayn KN: Adipose tissue as a buffer for daily lipid flux. Diabetologia 2002, 45(9):1201-1210.

14. Manolopoulos KN, Karpe F, Frayn KN: Gluteofemoral body fat as a determinant of metabolic health. Int J Obes 2010, 34(6):949-959.

15. Timmers S, Konings E, Bilet L, Houtkooper RH, van de Weijer T, Goossens GH, Hoeks J, van der Krieken S, Ryu D, Kersten S et al: Calorie restriction-like effects of 30 days of resveratrol supplementation on energy metabolism and metabolic profile in obese humans. Cell metabolism 2011, 14(5):612-622.

16. Brasnyo P, Molnar GA, Mohas M, Marko L, Laczy B, Cseh J, Mikolas E, Szijarto IA, Merei A, Halmai R et al: Resveratrol improves insulin sensitivity, reduces oxidative stress and activates the Akt pathway in type 2 diabetic patients. The British journal of nutrition 2011, 106(3):383-389.

17. Bogdanski P, Suliburska J, Szulinska M, Stepien M, Pupek-Musialik D, Jablecka A: Green tea extract reduces blood pressure, inflammatory biomarkers, and oxidative stress and improves parameters associated with insulin resistance in obese, hypertensive patients. Nutrition research 2012, 32(6):421-427.

18. Chen IJ, Liu C-Y, Chiu J-P, Hsu C-H: Therapeutic effect of high-dose green tea extract on weight reduction: A randomized, double-blind, placebo-controlled clinical trial. Clinical nutrition 2016, 35(3):592-599. 
19. Konings E, Timmers S, Boekschoten MV, Goossens GH, Jocken JW, Afman LA, Muller M, Schrauwen P, Mariman EC, Blaak EE: The effects of 30 days resveratrol supplementation on adipose tissue morphology and gene expression patterns in obese men. International journal of obesity 2014, 38(3):470-473.

20. Olholm J, Paulsen SK, Cullberg KB, Richelsen B, Pedersen SB: Anti-inflammatory effect of resveratrol on adipokine expression and secretion in human adipose tissue explants. Int J Obes 2010, 34(10):1546-1553.

21. Skurk T, Alberti-Huber C, Herder C, Hauner H: Relationship between adipocyte size and adipokine expression and secretion. The Journal of clinical endocrinology and metabolism 2007, 92(3):10231033.

22. Michaud A, Boulet MM, Veilleux A, Noel S, Paris G, Tchernof A: Abdominal subcutaneous and omental adipocyte morphology and its relation to gene expression, lipolysis and adipocytokine levels in women. Metabolism: clinical and experimental 2014, 63(3):372-381.

23. Ramirez-Zacarias JL, Castro-Munozledo F, Kuri-Harcuch W: Quantitation of adipose conversion and triglycerides by staining intracytoplasmic lipids with Oil red O. Histochemistry 1992, 97(6):493-497.

24. Moon HS, Chung CS, Lee HG, Kim TG, Choi YJ, Cho CS: Inhibitory effect of (-)-epigallocatechin3-gallate on lipid accumulation of 3T3-L1 cells. Obesity 2007, 15(11):2571-2582.

25. Kim HK, Della-Fera M, Lin J, Baile CA: Docosahexaenoic acid inhibits adipocyte differentiation and induces apoptosis in 3T3-L1 preadipocytes. The Journal of nutrition 2006, 136(12):2965-2969.

26. Kawada T, Aoki N, Kamei Y, Maeshige K, Nishiu S, Sugimoto E: Comparative investigation of vitamins and their analogues on terminal differentiation, from preadipocytes to adipocytes, of 3T3L1 cells. Comparative biochemistry and physiologyA, Comparative physiology 1990, 96(2):323326.

27. Rayalam S, Yang JY, Ambati S, Della-Fera MA, Baile CA: Resveratrol induces apoptosis and inhibits adipogenesis in 3T3-L1 adipocytes. Phytotherapy research : PTR 2008, 22(10):1367-1371.

28. Dubois SG, Heilbronn LK, Smith SR, Albu JB, Kelley DE, Ravussin E: Decreased expression of adipogenic genes in obese subjects with type 2 diabetes. Obesity 2006, 14(9):1543-1552.

29. Jocken JW, Langin D, Smit E, Saris WH, Valle C, Hul GB, Holm C, Arner P, Blaak EE: Adipose triglyceride lipase and hormone-sensitive lipase protein expression is decreased in the obese insulin-resistant state. The Journal of clinical endocrinology and metabolism 2007, 92(6):22922299.

30. Chen S, Li Z, Li W, Shan Z, Zhu W: Resveratrol inhibits cell differentiation in 3T3-L1 adipocytes via activation of AMPK. Canadian journal of physiology and pharmacology 2011, 89(11):793-799.

31. Fischer-Posovszky P, Kukulus V, Tews D, Unterkircher T, Debatin KM, Fulda S, Wabitsch M: Resveratrol regulates human adipocyte number and function in a Sirt1-dependent manner. Am J Clin Nutr 2010, 92(1):5-15.

32. Kouidhi S, Berrhouma R, Rouissi K, Jarboui S, Clerget-Froidevaux MS, Seugnet I, Bchir F, Demeneix B, Guissouma H, Elgaaied AB: Human subcutaneous adipose tissue Glut 4 mRNA expression in obesity and type 2 diabetes. Acta diabetologica 2013, 50(2):227-232.

33. Smith U, Kahn BB: Adipose tissue regulates insulin sensitivity: role of adipogenesis, de novo lipogenesis and novel lipids. Journal of internal medicine 2016, 280(5):465-475.

34. Lasa A, Schweiger M, Kotzbeck P, Churruca I, Simon E, Zechner R, Portillo MP: Resveratrol regulates lipolysis via adipose triglyceride lipase. The Journal of nutritional biochemistry 2012, 23(4):379-384.

35. Shan T, Ren Y, Wang Y: Sirtuin 1 affects the transcriptional expression of adipose triglyceride lipase in porcine adipocytes. Journal of animal science 2013, 91(3):1247-1254.

36. Girousse A, Tavernier G, Valle C, Moro C, Mejhert N, Dinel A-L, Houssier M, Roussel B, BessePatin A, Combes $\mathrm{M}$ et al: Partial Inhibition of Adipose Tissue Lipolysis Improves Glucose Metabolism and Insulin Sensitivity Without Alteration of Fat Mass. PLoS biology 2013, 11(2):e1001485.

37. Schweiger M, Romauch M, Schreiber R, Grabner GF, Hütter S, Kotzbeck P, Benedikt P, Eichmann TO, Yamada S, Knittelfelder O et al: Pharmacological inhibition of adipose triglyceride lipase corrects high-fat diet-induced insulin resistance and hepatosteatosis in mice. Nature communications 2017, 8:14859. 
38. Madsen L, Petersen RK, Kristiansen K: Regulation of adipocyte differentiation and function by polyunsaturated fatty acids. Biochimica et biophysica acta 2005, 1740(2):266-286.

39. Lee MJ, Fried SK: Optimal protocol for the differentiation and metabolic analysis of human adipose stromal cells. Methods in enzymology 2014, 538:49-65.

40. Lee MH, Goralczyk AG, Kriszt R, Ang XM, Badowski C, Li Y, Summers SA, Toh SA, Yassin MS, Shabbir A et al: ECM microenvironment unlocks brown adipogenic potential of adult human bone marrow-derived MSCs. Scientific reports 2016, 6:21173.

41. Aubin K, Safoine M, Proulx M, Audet-Casgrain M-A, Côté J-F, Têtu F-A, Roy A, Fradette J: Characterization of $<$ italic $>$ In Vitro $<$ italic $>$ Engineered Human Adipose Tissues: Relevant Adipokine Secretion and Impact of TNF- $\alpha$. PloS one 2015, 10(9):e0137612.

42. Rosenow A, Noben JP, Jocken J, Kallendrusch S, Fischer-Posovszky P, Mariman EC, Renes J: Resveratrol-induced changes of the human adipocyte secretion profile. Journal of proteome research 2012, 11(9):4733-4743.

43. Li S, Bouzar C, Cottet-Rousselle C, Zagotta I, Lamarche F, Wabitsch M, Tokarska-Schlattner M, Fischer-Posovszky P, Schlattner U, Rousseau D: Resveratrol inhibits lipogenesis of 3T3-L1 and SGBS cells by inhibition of insulin signaling and mitochondrial mass increase. Biochimica et Biophysica Acta (BBA) - Bioenergetics 2016, 1857(6):643-652.

44. Poulsen MM, Vestergaard PF, Clasen BF, Radko Y, Christensen LP, Stodkilde-Jorgensen H, Moller N, Jessen N, Pedersen SB, Jorgensen JO: High-dose resveratrol supplementation in obese men: an investigator-initiated, randomized, placebo-controlled clinical trial of substrate metabolism, insulin sensitivity, and body composition. Diabetes 2013, 62(4):1186-1195.

45. Yoshino J, Conte C, Fontana L, Mittendorfer B, Imai S, Schechtman KB, Gu C, Kunz I, Rossi Fanelli F, Patterson BW et al: Resveratrol supplementation does not improve metabolic function in nonobese women with normal glucose tolerance. Cell metabolism 2012, 16(5):658-664.

46. Janssens PL, Hursel R, Westerterp-Plantenga MS: Long-term green tea extract supplementation does not affect fat absorption, resting energy expenditure, and body composition in adults. The Journal of nutrition 2015, 145(5):864-870.

47. Mahler A, Steiniger J, Bock M, Klug L, Parreidt N, Lorenz M, Zimmermann BF, Krannich A, Paul F, Boschmann M: Metabolic response to epigallocatechin-3-gallate in relapsing-remitting multiple sclerosis: a randomized clinical trial. Am J Clin Nutr 2015, 101(3):487-495.

48. Ahmed B, Liu S, Si H: Antiadipogenic Effects and Mechanisms of Combinations of Genistein, Epigallocatechin-3-Gallate, and/or Resveratrol in Preadipocytes. Journal of medicinal food 2016.

49. Virtue S, Vidal-Puig A: Adipose tissue expandability, lipotoxicity and the Metabolic Syndrome-an allostatic perspective. Biochimica et biophysica acta 2010, 1801(3):338-349.

50. Sun K, Tordjman J, Clement K, Scherer PE: Fibrosis and adipose tissue dysfunction. Cell metabolism 2013, 18(4):470-477.

51. Weisberg SP, McCann D, Desai M, Rosenbaum M, Leibel RL, Ferrante AW, Jr.: Obesity is associated with macrophage accumulation in adipose tissue. The Journal of clinical investigation 2003, 112(12):1796-1808.

52. Mraz M, Haluzik M: The role of adipose tissue immune cells in obesity and low-grade inflammation. The Journal of endocrinology 2014, 222(3):R113-127.

53. Bluher M: Adipose tissue inflammation: a cause or consequence of obesity-related insulin resistance? Clinical science 2016, 130(18):1603-1614.

54. Yang Q, Graham TE, Mody N, Preitner F, Peroni OD, Zabolotny JM, Kotani K, Quadro L, Kahn BB: Serum retinol binding protein 4 contributes to insulin resistance in obesity and type 2 diabetes. Nature 2005, 436(7049):356-362.

55. Aguirre L, Fernández-Quintela A, Arias N, Portillo M: Resveratrol: Anti-Obesity Mechanisms of Action. Molecules 2014, 19(11):18632.

56. Canto C, Auwerx J: PGC-1alpha, SIRT1 and AMPK, an energy sensing network that controls energy expenditure. Current opinion in lipidology 2009, 20(2):98-105.

57. Wang S, Moustaid-Moussa N, Chen L, Mo H, Shastri A, Su R, Bapat P, Kwun I, Shen CL: Novel insights of dietary polyphenols and obesity. The Journal of nutritional biochemistry 2014, 25(1):118. 
58. Kopecky J, Rossmeisl M, Flachs P, Kuda O, Brauner P, Jilkova Z, Stankova B, Tvrzicka E, Bryhn M: n-3 PUFA: bioavailability and modulation of adipose tissue function. The Proceedings of the Nutrition Society 2009, 68(4):361-369.

59. Azhar Y, Parmar A, Miller CN, Samuels JS, Rayalam S: Phytochemicals as novel agents for the induction of browning in white adipose tissue. Nutrition \& metabolism 2016, 13:89.

60. Siriwardhana N, Kalupahana NS, Cekanova M, LeMieux M, Greer B, Moustaid-Moussa N: Modulation of adipose tissue inflammation by bioactive food compounds. The Journal of nutritional biochemistry 2013, 24(4):613-623.

61. Fain JN, Madan AK, Hiler ML, Cheema P, Bahouth SW: Comparison of the release of adipokines by adipose tissue, adipose tissue matrix, and adipocytes from visceral and subcutaneous abdominal adipose tissues of obese humans. Endocrinology 2004, 145(5):2273-2282.

62. Bastard JP, Maachi M, Lagathu C, Kim MJ, Caron M, Vidal H, Capeau J, Feve B: Recent advances in the relationship between obesity, inflammation, and insulin resistance. European cytokine network 2006, 17(1):4-12.

63. Gouranton E, Thabuis C, Riollet C, Malezet-Desmoulins C, El Yazidi C, Amiot MJ, Borel P, Landrier JF: Lycopene inhibits proinflammatory cytokine and chemokine expression in adipose tissue. The Journal of nutritional biochemistry 2011, 22(7):642-648.

64. Zagotta I, Dimova EY, Funcke JB, Wabitsch M, Kietzmann T, Fischer-Posovszky P: Resveratrol suppresses PAI-1 gene expression in a human in vitro model of inflamed adipose tissue. Oxidative medicine and cellular longevity 2013, 2013:793525.

65. Romacho T, Glosse P, Richter I, Elsen M, Schoemaker MH, van Tol EA, Eckel J: Nutritional ingredients modulate adipokine secretion and inflammation in human primary adipocytes. Nutrients 2015, 7(2):865-886.

66. Kim S, Jin Y, Choi Y, Park T: Resveratrol exerts anti-obesity effects via mechanisms involving down-regulation of adipogenic and inflammatory processes in mice. Biochemical pharmacology 2011, 81(11):1343-1351.

67. Kalupahana NS, Claycombe K, Newman SJ, Stewart T, Siriwardhana N, Matthan N, Lichtenstein AH, Moustaid-Moussa N: Eicosapentaenoic acid prevents and reverses insulin resistance in highfat diet-induced obese mice via modulation of adipose tissue inflammation. The Journal of nutrition 2010, 140(11):1915-1922.

68. Figueras M, Olivan M, Busquets S, López-Soriano FJ, Argilés JM: Effects of Eicosapentaenoic Acid (EPA) Treatment on Insulin Sensitivity in an Animal Model of Diabetes: Improvement of the Inflammatory Status. Obesity 2011, 19(2):362-369.

69. LeMieux MJ, Kalupahana NS, Scoggin S, Moustaid-Moussa N: Eicosapentaenoic acid reduces adipocyte hypertrophy and inflammation in diet-induced obese mice in an adiposity-independent manner. The Journal of nutrition 2015, 145(3):411-417.

70. Itariu BK, Zeyda M, Hochbrugger EE, Neuhofer A, Prager G, Schindler K, Bohdjalian A, Mascher D, Vangala S, Schranz M et al: Long-chain n-3 PUFAs reduce adipose tissue and systemic inflammation in severely obese nondiabetic patients: a randomized controlled trial. Am J Clin Nutr 2012, 96(5):1137-1149.

71. Li K, Huang T, Zheng J, Wu K, Li D: Effect of marine-derived n-3 polyunsaturated fatty acids on C-reactive protein, interleukin 6 and tumor necrosis factor alpha: a meta-analysis. PloS one 2014, 9(2):e88103.

72. McEneny J, Wade L, Young IS, Masson L, Duthie G, McGinty A, McMaster C, Thies F: Lycopene intervention reduces inflammation and improves HDL functionality in moderately overweight middle-aged individuals. The Journal of nutritional biochemistry 2013, 24(1):163-168.

73. Li YF, Chang YY, Huang HC, Wu YC, Yang MD, Chao PM: Tomato juice supplementation in young women reduces inflammatory adipokine levels independently of body fat reduction. Nutrition 2015, 31(5):691-696.

74. Oh DY, Talukdar S, Bae EJ, Imamura T, Morinaga H, Fan W, Li P, Lu WJ, Watkins SM, Olefsky JM: GPR120 is an omega-3 fatty acid receptor mediating potent anti-inflammatory and insulinsensitizing effects. Cell 2010, 142(5):687-698.

75. Polus A, Kiec-Wilk B, Razny U, Gielicz A, Schmitz G, Dembinska-Kiec A: Influence of dietary fatty acids on differentiation of human stromal vascular fraction preadipocytes. Biochimica et biophysica acta 2015, 1851(9):1146-1155. 
76. Masoodi M, Kuda O, Rossmeisl M, Flachs P, Kopecky J: Lipid signaling in adipose tissue: Connecting inflammation \& metabolism. Biochimica et biophysica acta 2015, 1851(4):503-518.

77. Gonzales AM, Orlando RA: Curcumin and resveratrol inhibit nuclear factor-kappaB-mediated cytokine expression in adipocytes. Nutrition \& metabolism 2008, 5:17.

78. Hadad N, Levy R: The synergistic anti-inflammatory effects of lycopene, lutein, $\beta$-carotene, and carnosic acid combinations via redox-based inhibition of NF- $\mathrm{kB}$ signaling. Free Radical Biology and Medicine 2012, 53(7):1381-1391.

79. Siriwardhana N, Kalupahana NS, Fletcher S, Xin W, Claycombe KJ, Quignard-Boulange A, Zhao L, Saxton AM, Moustaid-Moussa N: n-3 and n-6 polyunsaturated fatty acids differentially regulate adipose angiotensinogen and other inflammatory adipokines in part via NF-kappaB-dependent mechanisms. The Journal of nutritional biochemistry 2012, 23(12):1661-1667.

80. Zagotta I, Dimova EY, Debatin KM, Wabitsch M, Kietzmann T, Fischer-Posovszky P: Obesity and inflammation: reduced cytokine expression due to resveratrol in a human in vitro model of inflamed adipose tissue. Frontiers in pharmacology 2015, 6:79.

81. Berg AH, Lin Y, Lisanti MP, Scherer PE: Adipocyte differentiation induces dynamic changes in NF-kappaB expression and activity. American journal of physiology Endocrinology and metabolism 2004, 287(6):E1178-1188.

82. Kanda H, Tateya S, Tamori Y, Kotani K, Hiasa K, Kitazawa R, Kitazawa S, Miyachi H, Maeda S, Egashira K et al: MCP-1 contributes to macrophage infiltration into adipose tissue, insulin resistance, and hepatic steatosis in obesity. The Journal of clinical investigation 2006, 116(6):14941505.

83. Ota T: Chemokine systems link obesity to insulin resistance. Diabetes \& metabolism journal 2013, 37(3):165-172.

84. Luvizotto RdAM, Nascimento AF, Imaizumi E, Pierine DT, Conde SJ, Correa CR, Yeum K-J, Ferreira ALA: Lycopene supplementation modulates plasma concentrations and epididymal adipose tissue mRNA of leptin, resistin and IL-6 in diet-induced obese rats. British Journal of Nutrition 2013, 110(10):1803-1809.

85. Tishinsky JM, Ma DW, Robinson LE: Eicosapentaenoic acid and rosiglitazone increase adiponectin in an additive and PPARgamma-dependent manner in human adipocytes. Obesity 2011, 19(2):262-268.

86. Zhang HY, Du ZX, Meng X: Resveratrol prevents TNFalpha-induced suppression of adiponectin expression via PPARgamma activation in 3T3-L1 adipocytes. Clinical and experimental medicine 2013, 13(3):193-199.

87. Beaudoin MS, Snook LA, Arkell AM, Simpson JA, Holloway GP, Wright DC: Resveratrol supplementation improves white adipose tissue function in a depot-specific manner in Zucker diabetic fatty rats. American journal of physiology Regulatory, integrative and comparative physiology 2013, 305(5):R542-551.

88. Faghihzadeh F, Adibi P, Rafiei R, Hekmatdoost A: Resveratrol supplementation improves inflammatory biomarkers in patients with nonalcoholic fatty liver disease. Nutrition research 2014, 34(10):837-843.

89. Chen YK, Cheung C, Reuhl KR, Liu AB, Lee MJ, Lu YP, Yang CS: Effects of green tea polyphenol (-)-epigallocatechin-3-gallate on newly developed high-fat/Western-style diet-induced obesity and metabolic syndrome in mice. Journal of agricultural and food chemistry 2011, 59(21):1186211871.

90. Huh JY, Park YJ, Ham M, Kim JB: Crosstalk between Adipocytes and Immune Cells in Adipose Tissue Inflammation and Metabolic Dysregulation in Obesity. Molecules and Cells 2014, 37(5):365-371.

91. Luisa Bonet M, Canas JA, Ribot J, Palou A: Carotenoids and their conversion products in the control of adipocyte function, adiposity and obesity. Archives of biochemistry and biophysics 2015, 572:112-125.

92. Lagouge M, Argmann C, Gerhart-Hines Z, Meziane H, Lerin C, Daussin F, Messadeq N, Milne J, Lambert $\mathrm{P}$, Elliott $\mathrm{P}$ et al: Resveratrol improves mitochondrial function and protects against metabolic disease by activating SIRT1 and PGC-1alpha. Cell 2006, 127(6):1109-1122. 
93. Baur JA, Pearson KJ, Price NL, Jamieson HA, Lerin C, Kalra A, Prabhu VV, Allard JS, LopezLluch G, Lewis K et al: Resveratrol improves health and survival of mice on a high-calorie diet. Nature 2006, 444(7117):337-342.

94. Lu H, Meng X, Yang CS: Enzymology of methylation of tea catechins and inhibition of catecholO-methyltransferase by (-)-epigallocatechin gallate. Drug metabolism and disposition: the biological fate of chemicals 2003, 31(5):572-579.

95. Li Y, Zhao S, Zhang W, Zhao P, He B, Wu N, Han P: Epigallocatechin-3-O-gallate (EGCG) attenuates FFAs-induced peripheral insulin resistance through AMPK pathway and insulin signaling pathway in vivo. Diabetes research and clinical practice 2011, 93(2):205-214.

96. Moreno MF, De Laquila R, Okuda MH, Lira FS, de Souza GI, de Souza CT, Telles MM, Ribeiro $\mathrm{EB}$, do Nascimento CM, Oyama LM: Metabolic profile response to administration of epigallocatechin-3-gallate in high-fat-fed mice. Diabetology \& metabolic syndrome 2014, 6(1):84.

97. Dulloo AG, Duret C, Rohrer D, Girardier L, Mensi N, Fathi M, Chantre P, Vandermander J: Efficacy of a green tea extract rich in catechin polyphenols and caffeine in increasing 24-h energy expenditure and fat oxidation in humans. Am J Clin Nutr 1999, 70(6):1040-1045.

98. Most J, Goossens GH, Jocken JW, Blaak EE: Short-term supplementation with a specific combination of dietary polyphenols increases energy expenditure and alters substrate metabolism in overweight subjects. International journal of obesity 2014, 38(5):698-706.

99. Meex RCR, Schrauwen-Hinderling VB, Moonen-Kornips E, Schaart G, Mensink M, Phielix E, van de Weijer T, Sels J-P, Schrauwen P, Hesselink MKC: Restoration of Muscle Mitochondrial Function and Metabolic Flexibility in Type 2 Diabetes by Exercise Training Is Paralleled by Increased Myocellular Fat Storage and Improved Insulin Sensitivity. Diabetes 2010, 59(3):572.

100. Phielix E, Meex R, Ouwens DM, Sparks L, Hoeks J, Schaart G, Moonen-Kornips E, Hesselink MK, Schrauwen P: High oxidative capacity due to chronic exercise training attenuates lipidinduced insulin resistance. Diabetes 2012, 61(10):2472-2478.

101. Toth PP: Triglyceride-rich lipoproteins as a causal factor for cardiovascular disease. Vascular health and risk management 2016, 12:171-183.

102. Liu K, Zhou R, Wang B, Chen K, Shi LY, Zhu JD, Mi MT: Effect of green tea on glucose control and insulin sensitivity: a meta-analysis of 17 randomized controlled trials. Am J Clin Nutr 2013, 98(2):340-348.

103. Liu K, Zhou R, Wang B, Mi MT: Effect of resveratrol on glucose control and insulin sensitivity: a meta-analysis of 11 randomized controlled trials. Am J Clin Nutr 2014, 99(6):1510-1519.

104. Christenson J, Whitby SJ, Mellor D, Thomas J, McKune A, Roach PD, Naumovski N: The Effects of Resveratrol Supplementation in Overweight and Obese Humans: A Systematic Review of Randomized Trials. Metabolic syndrome and related disorders 2016, 14(7):323-333.

105. Mielgo-Ayuso J, Barrenechea L, Alcorta P, Larrarte E, Margareto J, Labayen I: Effects of dietary supplementation with epigallocatechin-3-gallate on weight loss, energy homeostasis, cardiometabolic risk factors and liver function in obese women: randomised, double-blind, placebo-controlled clinical trial. The British journal of nutrition 2014, 111(7):1263-1271.

106. van der Made SM, Plat J, Mensink RP: Resveratrol does not influence metabolic risk markers related to cardiovascular health in overweight and slightly obese subjects: a randomized, placebocontrolled crossover trial. PloS one 2015, 10(3):e0118393.

107. Tome-Carneiro J, Gonzalvez M, Larrosa M, Yanez-Gascon MJ, Garcia-Almagro FJ, Ruiz-Ros JA, Garcia-Conesa MT, Tomas-Barberan FA, Espin JC: One-year consumption of a grape nutraceutical containing resveratrol improves the inflammatory and fibrinolytic status of patients in primary prevention of cardiovascular disease. The American journal of cardiology 2012, 110(3):356-363.

108. Szkudelski T, Szkudelska K: Resveratrol and diabetes: from animal to human studies. Biochimica et biophysica acta 2015, 1852(6):1145-1154.

109. Tabrez S, Priyadarshini M, Urooj M, Shakil S, Ashraf GM, Khan MS, Kamal MA, Alam Q, Jabir NR, Abuzenadah AM et al: Cancer chemoprevention by polyphenols and their potential application as nanomedicine. Journal of environmental science and health Part C, Environmental carcinogenesis \& ecotoxicology reviews 2013, 31(1):67-98.

110. Jain SS, Paglialunga S, Vigna C, Ludzki A, Herbst EA, Lally JS, Schrauwen P, Hoeks J, Tupling AR, Bonen A et al: High-fat diet-induced mitochondrial biogenesis is regulated by mitochondrialderived reactive oxygen species activation of CaMKII. Diabetes 2014, 63(6):1907-1913. 
111. Egan B, Zierath JR: Exercise metabolism and the molecular regulation of skeletal muscle adaptation. Cell metabolism 2013, 17(2):162-184.

112. Anderson EJ, Lustig ME, Boyle KE, Woodlief TL, Kane DA, Lin CT, Price JW, 3rd, Kang L, Rabinovitch PS, Szeto HH et al: Mitochondrial H2O2 emission and cellular redox state link excess fat intake to insulin resistance in both rodents and humans. The Journal of clinical investigation 2009, 119(3):573-581.

113. Fisher-Wellman KH, Weber TM, Cathey BL, Brophy PM, Gilliam LA, Kane CL, Maples JM, Gavin TP, Houmard JA, Neufer PD: Mitochondrial respiratory capacity and content are normal in young insulin-resistant obese humans. Diabetes 2014, 63(1):132-141.

114. Chattopadhyay M, Khemka VK, Chatterjee G, Ganguly A, Mukhopadhyay S, Chakrabarti S: Enhanced ROS production and oxidative damage in subcutaneous white adipose tissue mitochondria in obese and type 2 diabetes subjects. Molecular and cellular biochemistry 2015, 399(1-2):95-103.

115. Di Meo S, Iossa S, Venditti P: Skeletal muscle insulin resistance: role of mitochondria and other ROS sources. The Journal of endocrinology 2017, 233(1):R15-r42.

116. Hirzel E, Lindinger PW, Maseneni S, Giese M, Rhein VV, Eckert A, Hoch M, Krahenbuhl S, Eberle AN: Differential modulation of ROS signals and other mitochondrial parameters by the antioxidants MitoQ, resveratrol and curcumin in human adipocytes. Journal of receptor and signal transduction research 2013, 33(5):304-312.

117. Casanova E, Baselga-Escudero L, Ribas-Latre A, Arola-Arnal A, Blade C, Arola L, Salvado MJ: Epigallocatechin gallate counteracts oxidative stress in docosahexaenoxic acid-treated myocytes. Biochimica et biophysica acta 2014, 1837(6):783-791.

118. Montgomery MK, Turner N: Mitochondrial dysfunction and insulin resistance: an update. Endocrine connections 2015, 4(1):R1-R15.

119. Phielix E, Schrauwen-Hinderling VB, Mensink M, Lenaers E, Meex R, Hoeks J, Kooi ME, Moonen-Kornips E, Sels JP, Hesselink MK et al: Lower intrinsic ADP-stimulated mitochondrial respiration underlies in vivo mitochondrial dysfunction in muscle of male type 2 diabetic patients. Diabetes 2008, 57(11):2943-2949.

120. Amati F, Dubé JJ, Coen PM, Stefanovic-Racic M, Toledo FGS, Goodpaster BH: Physical Inactivity and Obesity Underlie the Insulin Resistance of Aging. Diabetes care 2009, 32(8):1547.

121. Timmers S, de Ligt M, Phielix E, van de Weijer T, Hansen J, Moonen-Kornips E, Schaart G, Kunz I, Hesselink MK, Schrauwen-Hinderling VB et al: Resveratrol as Add-on Therapy in Subjects With Well-Controlled Type 2 Diabetes: A Randomized Controlled Trial. Diabetes care 2016, 39(12):2211-2217.

122. Hsu C-H, Tsai T-H, Kao Y-H, Hwang K-C, Tseng T-Y, Chou P: Effect of green tea extract on obese women: A randomized, double-blind, placebo-controlled clinical trial. Clinical nutrition 2008, 27(3):363-370.

123. Roberts JD, Roberts MG, Tarpey MD, Weekes JC, Thomas CH: The effect of a decaffeinated green tea extract formula on fat oxidation, body composition and exercise performance. J Int Soc Sports Nutr 2015, 12(1):1.

124. Gahreman D, Heydari M, Boutcher Y, Freund J, Boutcher S: The Effect of Green Tea Ingestion and Interval Sprinting Exercise on the Body Composition of Overweight Males: A Randomized Trial. Nutrients 2016, 8(8).

125. Holloszy JO, Coyle EF: Adaptations of skeletal muscle to endurance exercise and their metabolic consequences. Journal of applied physiology: respiratory, environmental and exercise physiology 1984, 56(4):831-838.

126. Most J, van Can JG, van Dijk JW, Goossens GH, Jocken J, Hospers JJ, Bendik I, Blaak EE: A 3day EGCG-supplementation reduces interstitial lactate concentration in skeletal muscle of overweight subjects. Scientific reports 2015, 5:17896.

127. Bosma M, Kersten S, Hesselink MK, Schrauwen P: Re-evaluating lipotoxic triggers in skeletal muscle: relating intramyocellular lipid metabolism to insulin sensitivity. Progress in lipid research 2012, 51(1):36-49.

128. Marinou K, Hodson L, Vasan SK, Fielding BA, Banerjee R, Brismar K, Koutsilieris M, Clark A, Neville MJ, Karpe F: Structural and functional properties of deep abdominal subcutaneous adipose 
tissue explain its association with insulin resistance and cardiovascular risk in men. Diabetes care 2014, 37(3):821-829.

129. Tchernof A, Despres JP: Pathophysiology of human visceral obesity: an update. Physiological reviews 2013, 93(1):359-404.

130. Lyons CL, Kennedy EB, Roche HM: Metabolic Inflammation-Differential Modulation by Dietary Constituents. Nutrients 2016, 8(5).

131. Wolfram S, Raederstorff D, Wang Y, Teixeira SR, Elste V, Weber P: TEAVIGO (epigallocatechin gallate) supplementation prevents obesity in rodents by reducing adipose tissue mass. Annals of Nutrition \& Metabolism 2005, 49(1):54-63.

132. Bose M, Lambert JD, Ju J, Reuhl KR, Shapses SA, Yang CS: The major green tea polyphenol, (-)epigallocatechin-3-gallate, inhibits obesity, metabolic syndrome, and fatty liver disease in high-fatfed mice. The Journal of nutrition 2008, 138(9):1677-1683.

133. Friedrich M, Petzke KJ, Raederstorff D, Wolfram S, Klaus S: Acute effects of epigallocatechin gallate from green tea on oxidation and tissue incorporation of dietary lipids in mice fed a high-fat diet. International journal of obesity 2012, 36(5):735-743.

134. Poulsen MM, Larsen JO, Hamilton-Dutoit S, Clasen BF, Jessen N, Paulsen SK, Kjaer TN, Richelsen B, Pedersen SB: Resveratrol up-regulates hepatic uncoupling protein 2 and prevents development of nonalcoholic fatty liver disease in rats fed a high-fat diet. Nutrition research 2012, 32(9):701-708.

135. Zhao W, Li A, Feng X, Hou T, Liu K, Liu B, Zhang N: Metformin and resveratrol ameliorate muscle insulin resistance through preventing lipolysis and inflammation in hypoxic adipose tissue. Cellular signalling 2016, 28(9):1401-1411.

136. Jans A, Sparks LM, van Hees AM, Gjelstad IM, Tierney AC, Riserus U, Drevon CA, Roche HM, Schrauwen P, Blaak EE: Transcriptional metabolic inflexibility in skeletal muscle among individuals with increasing insulin resistance. Obesity 2011, 19(11):2158-2166.

137. Storlien L, Oakes ND, Kelley DE: Metabolic flexibility. The Proceedings of the Nutrition Society 2004, 63(2):363-368.

138. Hoehn KL, Turner N, Swarbrick MM, Wilks D, Preston E, Phua Y, Joshi H, Furler SM, Larance M, Hegarty BD et al: Acute or chronic upregulation of mitochondrial fatty acid oxidation has no net effect on whole-body energy expenditure or adiposity. Cell metabolism 2010, 11(1):70-76.

139. Kapoor MP, Sugita M, Fukuzawa Y, Okubo T: Physiological effects of epigallocatechin-3-gallate (EGCG) on energy expenditure for prospective fat oxidation in humans: A systematic review and meta-analysis. The Journal of nutritional biochemistry 2016, 43:1-10.

140. Legeay S, Rodier M, Fillon L, Faure S, Clere N: Epigallocatechin Gallate: A Review of Its Beneficial Properties to Prevent Metabolic Syndrome. Nutrients 2015, 7(7):5443-5468.

141. Gambini J, Ingles M, Olaso G, Lopez-Grueso R, Bonet-Costa V, Gimeno-Mallench L, MasBargues C, Abdelaziz KM, Gomez-Cabrera MC, Vina J et al: Properties of Resveratrol: In Vitro and In Vivo Studies about Metabolism, Bioavailability, and Biological Effects in Animal Models and Humans. Oxidative medicine and cellular longevity 2015, 2015:837042.

142. Ullmann, Haller, Decourt, Girault, Spitzer, Weber: Plasma-Kinetic Characteristics of Purified and Isolated Green Tea Catechin Epigallocatechin Gallate (EGCG) after 10 Days Repeated Dosing in Healthy Volunteers. International Journal for Vitamin and Nutrition Research 2004, 74(4):269278.

143. Rhodes LE, Darby G, Massey KA, Clarke KA, Dew TP, Farrar MD, Bennett S, Watson RE, Williamson G, Nicolaou A: Oral green tea catechin metabolites are incorporated into human skin and protect against UV radiation-induced cutaneous inflammation in association with reduced production of pro-inflammatory eicosanoid 12-hydroxyeicosatetraenoic acid. The British journal of nutrition 2013, 110(5):891-900.

144. Carpene C, Gomez-Zorita S, Deleruyelle S, Carpene MA: Novel strategies for preventing diabetes and obesity complications with natural polyphenols. Current medicinal chemistry 2015, 22(1):150164.

145. Cohn W, Thurmann P, Tenter U, Aebischer C, Schierle J, Schalch W: Comparative multiple dose plasma kinetics of lycopene administered in tomato juice, tomato soup or lycopene tablets. European journal of nutrition 2004, 43(5):304-312. 
146. Maiani G, Caston MJ, Catasta G, Toti E, Cambrodon IG, Bysted A, Granado-Lorencio F, Olmedilla-Alonso B, Knuthsen P, Valoti M et al: Carotenoids: actual knowledge on food sources, intakes, stability and bioavailability and their protective role in humans. Molecular nutrition \& food research 2009, 53 Suppl 2:S194-218.

147. Yurko-Mauro K, Kralovec J, Bailey-Hall E, Smeberg V, Stark JG, Salem N, Jr.: Similar eicosapentaenoic acid and docosahexaenoic acid plasma levels achieved with fish oil or krill oil in a randomized double-blind four-week bioavailability study. Lipids in health and disease 2015, 14:99.

148. Bonet ML, Canas JA, Ribot J, Palou A: Carotenoids in Adipose Tissue Biology and Obesity. Subcellular biochemistry 2016, 79:377-414.

149. Wolfram S, Wang Y, Thielecke F: Anti-obesity effects of green tea: from bedside to bench. Molecular nutrition \& food research 2006, 50(2):176-187.

150. Shimoda H, Hitoe S, Nakamura S, Matsuda H: Purple Tea and Its Extract Suppress Diet-induced Fat Accumulation in Mice and Human Subjects by Inhibiting Fat Absorption and Enhancing Hepatic Carnitine Palmitoyltransferase Expression. International journal of biomedical science : IJBS 2015, 11(2):67-75.

151. Mendes KL, de Pinho L, Andrade JMO, Paraíso AF, Lula JF, Macedo SM, Feltenberger JD, Guimarães ALS, de Paula AMB, Santos SHS: Distinct metabolic effects of resveratrol on lipogenesis markers in mice adipose tissue treated with high-polyunsaturated fat and high-protein diets. Life sciences 2016, 153:66-73.

152. Naumovski N, Blades BL, Roach PD: Food Inhibits the Oral Bioavailability of the Major Green Tea Antioxidant Epigallocatechin Gallate in Humans. Antioxidants 2015, 4(2):373-393.

153. Ozdal T, Sela DA, Xiao J, Boyacioglu D, Chen F, Capanoglu E: The Reciprocal Interactions between Polyphenols and Gut Microbiota and Effects on Bioaccessibility. Nutrients 2016, 8(2):78.

154. Goh KP, Lee HY, Lau DP, Supaat W, Chan YH, Koh AF: Effects of resveratrol in patients with type 2 diabetes mellitus on skeletal muscle SIRT1 expression and energy expenditure. International journal of sport nutrition and exercise metabolism 2014, 24(1):2-13.

155. Thielecke F, Rahn G, Bohnke J, Adams F, Birkenfeld AL, Jordan J, Boschmann M: Epigallocatechin-3-gallate and postprandial fat oxidation in overweight/obese male volunteers: a pilot study. Eur J Clin Nutr 2010, 64(7):704-713.

156. Ristow M, Zarse K, Oberbach A, Kloting N, Birringer M, Kiehntopf M, Stumvoll M, Kahn CR, Bluher M: Antioxidants prevent health-promoting effects of physical exercise in humans. Proceedings of the National Academy of Sciences of the United States of America 2009, 106(21):8665-8670.

157. Lagarde M, Calzada C, Guichardant M, Vericel E: In vitro and in vivo bimodal effects of docosahexaenoic acid supplements on redox status and platelet function. Prostaglandins Leukot Essent Fatty Acids 2016, 108:1-4.

158. Arias N, Macarulla MT, Aguirre L, Milton I, Portillo MP: The combination of resveratrol and quercetin enhances the individual effects of these molecules on triacylglycerol metabolism in white adipose tissue. European journal of nutrition 2016, 55(1):341-348.

159. Canas JA, Damaso L, Altomare A, Killen K, Hossain J, Balagopal PB: Insulin resistance and adiposity in relation to serum beta-carotene levels. The Journal of pediatrics 2012, 161(1):5864.e51-52.

160. Lorente-Cebrian S, Costa AG, Navas-Carretero S, Zabala M, Martinez JA, Moreno-Aliaga MJ: Role of omega-3 fatty acids in obesity, metabolic syndrome, and cardiovascular diseases: a review of the evidence. Journal of physiology and biochemistry 2013, 69(3):633-651.

161. Calder PC, Ahluwalia N, Brouns F, Buetler T, Clement K, Cunningham K, Esposito K, Jonsson LS, Kolb H, Lansink M et al: Dietary factors and low-grade inflammation in relation to overweight and obesity. The British journal of nutrition 2011, 106 Suppl 3:S5-78.

162. Cao H: Adipocytokines in obesity and metabolic disease. The Journal of endocrinology 2014, 220(2):T47-59.

163. Chang CC, Lin KY, Peng KY, Day YJ, Hung LM: Resveratrol exerts anti-obesity effects in highfat diet obese mice and displays differential dosage effects on cytotoxicity, differentiation, and lipolysis in 3T3-L1 cells. Endocrine journal 2015. 
164. Pope BD, Warren CR, Parker KK, Cowan CA: Microenvironmental Control of Adipocyte Fate and Function. Trends in Cell Biology 2016, 26(10):745-755.

165. Ang XM, Lee MH, Blocki A, Chen C, Ong LL, Asada HH, Sheppard A, Raghunath M: Macromolecular crowding amplifies adipogenesis of human bone marrow-derived mesenchymal stem cells by enhancing the pro-adipogenic microenvironment. Tissue engineering Part A 2014, 20(5-6):966-981.

166. Baumann P, Spulber M, Fischer O, Car A, Meier W: Investigation of Horseradish Peroxidase Kinetics in an "Organelle-Like" Environment. Small 2017.

167. Zeiger AS, Loe FC, Li R, Raghunath M, Van Vliet KJ: Macromolecular Crowding Directs Extracellular Matrix Organization and Mesenchymal Stem Cell Behavior. PloS one 2012, 7(5):e37904.

168. Hinton BJ, Fan B, Ng BK, Shepherd JA: Dual energy X-ray absorptiometry body composition reference values of limbs and trunk from NHANES 1999-2004 with additional visualization methods. PloS one 2017, 12(3):e0174180.

169. Leblanc V, Hudon AM, Royer MM, Corneau L, Dodin S, Begin C, Lemieux S: Differences between men and women in dietary intakes and metabolic profile in response to a 12-week nutritional intervention promoting the Mediterranean diet. J Nutr Sci 2015, 4:e13.

170. Gonçalves CG, Glade MJ, Meguid MM: Review: Metabolically healthy obese individuals: Key protective factors. Nutrition 2016, 32:14-20.

171. Gardner C, Wylie-Rosett J, Gidding SS, Steffen LM, Johnson RK, Reader D, Lichtenstein AH: Nonnutritive sweeteners: current use and health perspectives: a scientific statement from the American Heart Association and the American Diabetes Association. Diabetes care 2012, 35(8):1798-1808.

172. Troesch B, Biesalski HK, Bos R, Buskens E, Calder PC, Saris WH, Spieldenner J, Verkade HJ, Weber P, Eggersdorfer M: Increased Intake of Foods with High Nutrient Density Can Help to Break the Intergenerational Cycle of Malnutrition and Obesity. Nutrients 2015, 7(7):6016-6037.

173. Zeevi D, Korem T, Zmora N, Israeli D, Rothschild D, Weinberger A, Ben-Yacov O, Lador D, Avnit-Sagi T, Lotan-Pompan M et al: Personalized Nutrition by Prediction of Glycemic Responses. Cell 2015, 163(5):1079-1094.

174. Suez J, Korem T, Zeevi D, Zilberman-Schapira G, Thaiss CA, Maza O, Israeli D, Zmora N, Gilad S, Weinberger A et al: Artificial sweeteners induce glucose intolerance by altering the gut microbiota. Nature 2014, 514(7521):181-186.

175. Graf D, Di Cagno R, Fåk F, Flint HJ, Nyman M, Saarela M, Watzl B: Contribution of diet to the composition of the human gut microbiota. Microbial Ecology in Health and Disease 2015, 26:10.3402/mehd.v3426.26164.

176. Pedersen HK, Gudmundsdottir V, Nielsen HB, Hyotylainen T, Nielsen T, Jensen BA, Forslund K, Hildebrand F, Prifti E, Falony G et al: Human gut microbes impact host serum metabolome and insulin sensitivity. Nature 2016, 535(7612):376-381.

177. Torres-Perez E, Valero M, Garcia-Rodriguez B, Gonzalez-Irazabal Y, Calmarza P, Calvo-Ruata L, Ortega C, Garcia-Sobreviela MP, Sanz-Paris A, Artigas JM et al: The FAT expandability (FATe) Project: Biomarkers to determine the limit of expansion and the complications of obesity. Cardiovascular diabetology 2015, 14:40.

178. Canas JA, Lochrie A, McGowan AG, Hossain J, Schettino C, Balagopal PB: Effects of Mixed Carotenoids on Adipokines and Abdominal Adiposity in Children: A Pilot Study. The Journal of clinical endocrinology and metabolism 2017.

179. Williams EJ, Baines KJ, Berthon BS, Wood LG: Effects of an Encapsulated Fruit and Vegetable Juice Concentrate on Obesity-Induced Systemic Inflammation: A Randomised Controlled Trial. Nutrients 2017, 9(2):116.

180. Obesity and overweight - Fact sheet No. 311

181. Lustig RH: Fructose: it's "alcohol without the buzz". Advances in nutrition 2013, 4(2):226-235.

182. Pektas MB, Sadi G, Akar F: Long-Term Dietary Fructose Causes Gender-Different Metabolic and Vascular Dysfunction in Rats: Modulatory Effects of Resveratrol. Cellular physiology and biochemistry : international journal of experimental cellular physiology, biochemistry, and pharmacology 2015, 37(4):1407-1420. 
183. Hokayem M, Blond E, Vidal H, Lambert K, Meugnier E, Feillet-Coudray C, Coudray C, Pesenti $\mathrm{S}$, Luyton C, Lambert-Porcheron S et al: Grape polyphenols prevent fructose-induced oxidative stress and insulin resistance in first-degree relatives of type 2 diabetic patients. Diabetes care 2013, 36(6):1454-1461.

184. Misciagna G, Del Pilar Diaz M, Caramia DV, Bonfiglio C, Franco I, Noviello MR, Chiloiro M, Abbrescia DI, Mirizzi A, Tanzi M et al: Effect of a Low Glycemic Index Mediterranean Diet on Non-Alcoholic Fatty Liver Disease. A Randomized Controlled Clinici Trial. The journal of nutrition, health \& aging 2017, 21(4):404-412.

185. Niebylski ML, Redburn KA, Duhaney T, Campbell NR: Healthy food subsidies and unhealthy food taxation: A systematic review of the evidence. Nutrition 2015, 31(6):787-795. 


\title{
APPENDICES
}

\author{
Summary \\ Zusammenfassung \\ Valorization \\ Acknowledgements / Danksagungen \\ Publications \\ Curriculum Vitae
}




\section{Introduction}

The well-balanced interplay of lipid storage and release (lipolysis) in human adipose tissue (AT) secures energy homeostasis in times of a nutrient surplus or food shortage, respectively. More recently, the constant technological progress led to a continuous availability of cheap, processed energy-dense foods and a dramatic reduction of physical activity. Their concomitant negative effects are the increasing accumulation of body fat causing obesity, which elevates the risk for various non-communicable diseases e.g., type-2-diabetes, non-alcoholic fatty liver disease, cardiovascular diseases, and several forms of cancer. Promoting life-style changes is the first defence line against increased body fat and metabolic dysfunctions such as insulin resistance, ectopic fat accumulation and chronic low-grade inflammation. However, apparently this approach cannot be effectively implemented and maintained in all individuals as shown by the on-going obesity epidemic. Therefore, further non-invasive strategies need to be explored that contribute to the reversal or prevention of these metabolic impairments and promote weight loss. This explains the numerous studies in recent decades on cellular and molecular processes underlying fat metabolism and on effects of nutrients on adipocyte functions (Chapter 1). In this thesis, we investigated the effects of nutritional bio-actives like trans-resveratrol (Res), epigallocatechin-3gallate (EGCG), (all-E)-lycopene (Lyc), and eicosapentaenoic acid (EPA) on adipocyte functions and metabolic profiles of overweight-obese humans.

\section{In-vitro study results}

To identify nutritional bio-actives that could modulate adipocyte functions (e.g., the accumulation of cytosolic lipid droplets (LDs) in differentiating adipocytes) valid in-vitro test systems are required. Therefore, we set-up an automated high content assay using fluorescent microscopy (ArrayScan ${ }^{\circledR}$ VTI reader) to quantify LDs in adipocytes (Chapter 2). The assay was validated with several dietary constituents (n-3 polyunsaturated fatty acids (PUFAs), carotenoids, polyphenols) by determining their influence on LD accumulation and the expression of adipogenic and lipogenic genes (RT-qPCR). n-3 PUFAs, beta-carotene and hydroxytyrosol strongly inhibited the rosiglitazone-stimulated lipid formation in murine adipocytes, whereas Lyc and EGCG showed a moderate and Res no inhibition of LD accumulation. The observed effects on the LD formation induced by the nutritional constituents correlated with the modulation of the adipogenic genes PPAR-gamma, C/EBP-alpha, and aP2. Hence, this in-vitro assay is a valuable substitute for the commonly used Oil Red O procedure, allowing a rapid automated improved quantification of changes in adipocyte LD parameters.

Human AT dysfunction, characterized by hypertrophic adipocytes, inflammation, and impaired adipogenesis, rather than an increased fat mass per se, is strongly associated with obesity-related 
metabolic diseases. Therefore, in Chapter 3 we investigated the impact of bio-active dietary constituents on functional features of in-vitro differentiated primary human adipocytes. The bioactives Lyc, EPA, and Res were tested for their individual or combined effects. In addition to the fluorescence assay, AT function-related gene expression and cyto-/adipokine secretion (LiquiChip ${ }^{\circledR}$ ) was measured. Treating differentiating human adipocytes for 14 days with the combinations Lyc/Res and EPA/Res significantly inhibited the LD formation. Increased PPARgamma mRNA levels and a decreased expression of lipogenic markers (LPL, FAS, GLUT-4) and PLIN-1 accompanied this reduced lipid accumulation. Furthermore, the secretion of proinflammatory cytokines (IL-6 and CCL2/MCP-1) and adiponectin was blunted by the treatment with these combinations. These data indicate that treatment with combinations of dietary bioactives during adipogenic differentiation can influence adipocyte function by affecting the balance between adipogenic, lipogenic and lipolytic gene expression, resulting in reduced lipid storage and a less inflammatory secretion profile. Here we showed for the first time that combining Lyc or EPA with Res partially augmented the effects of the single compounds on human adipocytes, supporting the concept of mutual interactions between nutrients.

It is described that human multi-potent adipose-derived stem (hMADS) cells retain interindividual genetic and metabolic donor characteristics, which could modify the effect of nutritional bio-actives. Thus, we studied based on the results of Chapter 3, whether the combination Lyc/Res affects functional adipocyte features (incl. lipolytic response) of in-vitro differentiated hMADS cells. The cells were isolated from abdominal subcutaneous white AT of age-matched lean and obese donors (Chapter 4). The combination Lyc/Res strongly reduced the LD accumulation in differentiated hMADS cells independently of the donor's characteristics. Interestingly, Res alone affected the storage of LDs to a similar extent as Lyc/Res. Moreover, Res and Lyc/Res inhibited the lipolytic response (glycerol release) under basal and stimulated (beta-adrenergic) conditions in differentiated hMADS cells. These functional changes were accompanied by a decreased expression of the adipogenic (PPAR-gamma, C/EBP-alpha), lipogenic (FAS, GLUT-4) and lipolytic (ATGL, HSL) genes. The IL-6 and adiponectin secretion was also diminished by Res and the combination, hence reflecting a reduced pro-inflammatory secretion profile. Taken together, the results described in chapter 4 corroborate that Res is a potent driver of improved functional features in in-vitro differentiated human adipocytes under the used differentiation conditions and the effects seem independent of the donor's characteristics (lean versus obese). Nevertheless, evidence indicates that combinations of bio-actives may be a more beneficial strategy (as also observed in chapter 3) for preventing or correcting AT dysfunction and obesity-associated 
metabolic diseases. This may be a result of the constituents targeting distinct pathways in white AT and other physiological pathways that affect metabolic health.

\section{In-vivo study results}

Based on the above-mentioned indications and metabolically beneficial results of a recently reported short-term clinical study (3 days) with combinations of polyphenols (Most et al. IJO 2014), we investigated in Chapter 5 and 6 whether long-term supplementation of EGCG and Res (282 and $80 \mathrm{mg} / \mathrm{d}$ ) had positive effects on tissue-specific insulin sensitivity, mitochondrial capacity in skeletal muscle, lipid metabolism, and metabolic profiles. Therefore, a randomized, placebocontrolled, double-blind clinical trial was conducted in overweight-obese, non-diabetic subjects (21 male, 21 female). The 12-week EGCG/Res supplementation increased the oxidative capacity in permeabilized muscle fibres (ex-vivo) and preserved fasting and postprandial whole-body fat oxidation (indirect calorimetry) compared to the PLA group, which showed a significant decline in fat oxidation. This was corroborated by an elevated expression of genes and proteins involved in mitochondrial respiration and energy metabolism (skeletal muscle biopsies taken before and after intervention). Furthermore, pre- and post-intervention measurements showed that the EGCG/Res supplementation prevented an increase in plasma triacylglycerol levels in the fasted state and during a high-fat mixed meal test. However, whole-body fat mass, energy expenditure, and the fasting plasma metabolic profile were not affected, although visceral AT mass (DEXA) tended to decrease after the intervention. Finally, these observed metabolic changes did not translate into improved peripheral, hepatic, and AT insulin sensitivity (2-step hyperinsulinemiceuglycemic clamp), but could in the long term prevent weight gain and the worsening of insulin resistance (Chapter 5).

Chapter 6 describes the effects of the 12-week EGCG/Res supplementation on the adipose tissue morphology and transcriptome. Before and after intervention, abdominal subcutaneous AT biopsies were collected for the assessment of adipocyte size, mitochondrial protein content and transcriptional profile. The present study showed that the EGCG/Res supplementation had no effect on mean adipocyte size, adipocyte size distribution, and AT lipolysis (microdialysis) compared with the PLA group. However, the transcriptome results suggest that the intervention may induce the suppression of gene sets related to adipogenesis and apoptosis/autophagy, inflammation, and the immune response in AT of overweight-obese men and women, indicative of a diminished adipocyte turnover and adipose tissue inflammation, respectively. 


\section{Conclusions}

The in-vitro and in-vivo human studies described in this thesis extend the knowledge on whether and how individual and combinations of nutritional bio-actives benefit AT function, insulin sensitivity, and metabolic profiles. However, effects of treatment with combinations of bio-actives on adipocyte features in-vitro e.g., on lipid accumulation and secretory profile, are influenced by the applied differentiation conditions and do not necessarily translate into changes in AT biology and energy metabolism in humans in-vivo. Nevertheless, an increased mitochondrial capacity in skeletal muscle and whole-body fat oxidation were observed in overweight-obese adults after 12week supplementation with EGCG/Res. Hence, despite the subtle metabolic improvements, which may slow the progression of insulin resistance, it remains to be further determined whether different dosages or metabolites of bio-actives, concomitant to lifestyle interventions and/or medical treatments, might elicit more favourable effects in the long-term. In summary, bio-actives have the potential to improve adipocyte functions and therefore the outcomes of lifestyle changes, although their effects may depend on health and weight status, the gut microbiota, environmental factors, and genetic predisposition. However, the application of bio-active supplements cannot compensate for an unhealthy lifestyle including overconsumption of energy-dense, micronutrient deprived food products and minimal physical activity (Chapter 7). 
Zusammenfassung 


\section{Einleitung}

Das ausgeklügelte Zusammenspiel von Fetteinlagerung und -freisetzung (Lipolyse) im Fettgewebe des Menschen sichert die Energie-Homöostasis bei einem Nahrungsüberangebot bzw. in Zeiten des Nahrungsmangels. Der technologische Fortschritt der letzten Jahrzehnte begünstigte eine ständige Verfügbarkeit von billigen, industriell verarbeiteten energiereichen Nahrungsmitteln und eine drastische Abnahme der körperlichen Aktivität. Daraus folgt eine stetige Zunahme des Gesamtkörperfettes, was zu Adipositas führt und somit das Risiko erhöht, an nichtübertragbaren Krankheiten wie Typ-2 Diabetes, nichtalkoholischer Fettleber, Herz-Kreislauf Beschwerden und verschiedenen Krebsformen zu erkranken. Die erste und wichtigste Massnahme, um eine Zunahme des Körperfettes und metabolische Fehlfunktionen, wie Insulinresistenz, ektopische Fettansammlung und chronische geringgradige Entzündung zu verhindern, ist die Änderung des Lebensstils. Jedoch scheint dies schwer umsetzbar zu sein, wie die sich weiterhin epidemisch ausbreitetende Fettleibigkeit zeigt. Aufgrund dessen sind zusätzliche Strategien notwendig, um diese metabolischen Störungen umzukehren oder zu verhindern und somit eine Gewichtsabnahme zu fördern. Dies erklärt die zahlreichen Studien, welche die zellulären und molekularen Grundlagen des Fettstoffwechsels und den Einfluss von Nährstoffen auf die Fettzellfunktionen untersuchten (Kapitel 1). In dieser Dissertation haben wir die Effekte von „bioaktiven“ Nahrungsbestandteilen wie z.B. trans-Resveratrol (Res), Epigallocatechin-3-gallat (EGCG), (allE)-Lycopin (Lyc), und Eicosapentaensäure (EPA) auf die Funktionen der Fettzellen und die metabolischen Profile von übergewichtigen Probanden untersucht.

\section{Resultate der in-vitro Studie}

Für die Identifizierung von bioaktiven Substanzen, welche Fettzellfunktionen - z.B. die Bildung von zytosolischen Lipidtröpfchen (LT) während der Adipozyten-Differenzierung - beeinflussen, braucht es valide in-vitro Testmethoden. Dafür haben wir einen neuen automatischen „highcontent“ Assay etabliert, welcher mittels Fluoreszenz-Mikroskopie (ArrayScan ${ }^{\circledR}$ VTI) die LT in Adipozyten quantifizieren kann (Kapitel 2). Zur Validierung wurden verschiedene Nahrungsbestandteile (mehrfach ungesättigte Fettsäuren (n-3 PUFAs), Carotenoide, Polyphenole) bezüglich ihres Einflusses auf die LT Bildung und auf die Expression adipogener und lipogener Gene (RT-qPCR) getestet. n-3 PUFAs, beta-Carotin und Hydroxytyrosol inhibierten die Rosiglitazon-stimulierte Lipidansammlung in murinen Fettzellen stark, wohingegen Lyc und EGCG eine moderate und Res keine Inhibition aufzeigten. Die Änderung der mRNA Level der adipogenen Marker PPAR-gamma, C/EBP-alpha und aP2 korrelieren mit der beobachteten Wirkung der bioaktiven Substanzen auf die LT Bildung. Somit ist dieser in-vitro Assay ein wertvoller Ersatz für die üblich verwendete Oil Red O Färbung, da er eine verbesserte, 
automatisierte und schnelle Quantifizierung von Lipidtröpfchen-Parametern in Fettzellen ermöglicht.

Die Fehlfunktion des Fettgewebes, gekennzeichnet durch hypertrophe Adipozyten, Entzündung und beeinträchtigte Adipogenese, ist im Menschen weniger mit einem erhöhten Körperfettanteil per se assoziiert als mit den die Adipositas begleitenden metabolischen Krankheiten. Deshalb haben wir in Kapitel 3 untersucht, welchen Einfluss bioaktive Nahrungsbestandteile auf die funktionellen Parameter von in-vitro differenzierten primären humanen Adipozyten haben. Die bioaktiven Substanzen Lyc, EPA, und Res wurden alleine oder in Kombination getestet. Zusätzlich zum Fluoreszenz-Assay wurde die Expression von Genen des Fettstoffwechsels und die Sekretion von Zyto-/Adipokinen (LiquiChip ${ }^{\circledR}$ ) gemessen. Die 14-tägige Behandlung von differenzierenden humanen Adipozyten mit den Kombinationen Lyc/Res und EPA/Res inhibierte signifikant die Bildung von LT. Erhöhte PPAR-gamma mRNA Level und eine verringerte Expression von lipogenen Markern (LPL, FAS, GLUT-4) und PLIN-1 begleiteten die reduzierte Lipidakkumulierung. Des Weiteren war die Sekretion von pro-inflammatorischen Zytokinen (IL6 und CCL2/MCP-1) und Adiponektin nach der Behandlung mit diesen Kombinationen abgeschwächt. Diese Daten weisen darauf hin, dass die Behandlung mit bioaktiven SubstanzKombinationen während der Adipozyten-Differenzierung die Balanz zwischen adipogener, lipogener und lipolytischer Genexpression beeinflussen, was in einer verringerten Lipidansammlung und einem weniger entzündlichen Sekretionsprofil resultiert und somit einer verbesserten Fettzellfunktion entspricht. Ausserdem zeigen wir hier zum ersten Mal, dass das Kombinieren von Lyc oder EPA mit Res die Effekte der Einzelsubstanzen auf menschliche Fettzellen verstärkt, was wiederum das Konzept gegenseitiger Wechselwirkungen zwischen Nährstoffen bekräftigt.

Es ist beschrieben, dass humane multipotente Stammzellen des Fettgewebes (hMADS Zellen) interindividuelle genetische und metabolische Eigenschaften der Spender beibehalten, was die Wirkung von Nahrungsbestandteilen beeinflussen könnte. Somit haben wir basierend auf den Resultaten von Kapitel 3 untersucht, ob die Kombination Lyc/Res funktionelle Fettzelleigenschaften (inkl. lipolytische Antwort) von in-vitro differenzierten hMADS Zellen modifiziert. Die Stammzellen wurden aus abdominalem subkutanem weissem Fettgewebe von altersgleichen schlanken und adipösen Spendern isoliert (Kapitel 4). Lyc/Res reduzierte, unabhängig von den Spendereigenschaften, die Lipidakkumulierung in differenzierten hMADS Zellen signifikant. Interessanterweise, zeigte Res allein eine ähnlich starke Wirkung auf die LT Bildung wie Lyc/Res. Ferner inhibierten Res and Lyc/Res die lipolytische Antwort (Glycerol Ausschüttung) in differenzierten hMADS Zellen unter basalen und stimulierten (beta-adrenergen) 
Bedingungen. Diese funktionellen Veränderungen wurden von einer veringerten Expression der adipogenen (PPAR-gamma, C/EBP-alpha), lipogenen (FAS, GLUT-4) und lipolytischen (ATGL, HSL) Gene begleitet. Die Sekretion von IL-6 und Adiponektin war durch die Behandlung mit Res und Lyc/Res gleichermassen vermindert, was somit ein geringeres pro-inflammatorisches Sekretionsprofil darstellt. Zusammengefasst bekräftigen die in Kapitel 4 beschriebenen Ergebnisse, dass Res eine wirksame Substanz ist, welche die funktionellen Eigenschaften der invitro differenzierten humanen Fettzellen unter den gegebenen Differenzierungsbedingungen positiv beeinflusst. Ausserdem scheint die Wirkung von Res unabhängig von den Spendereigenschaften (schlank versus adipös) zu sein. Nichtsdestotrotz gibt es wissenschaftliche Hinweise (wie auch in Kapitel 3 beobachtet), dass Kombinationen von bioaktiven Nahrungsbestandteilen geeigneter sein könnten, um Fettgewebefehlfunktionen und Adipositasassoziierte metabolische Erkrankungen zu verhindern oder zu behandeln. Dies kann dadurch bedingt sein, dass die Substanzen auf unterschiedliche Funktionen des weissen Fettgewebes und auf andere, die metabolische Gesundheit beeinflussende Signalwege abzielen.

\section{Resultate der in-vivo Studie}

Basierend auf oben genannten Hinweisen und auf einer jüngst veröffentlichten Kurzzeit-Studie (3 Tage), welche positive metabolische Effekte von Polyphenol-Kombinationen beschrieb (Most et al. IJO 2014), untersuchten wir in Kapitel 5 und 6, ob eine Langzeit-Supplementierung mit EGCG und Res (282 und $80 \mathrm{mg} / \mathrm{d}$ ) eine vorteilhafte Wirkung auf die gewebespezifische Insulinsensitivität, die mitochondriale Kapazität im Muskel, den Lipid-Stoffwechsel und metabolische Profile hat. Dafür wurde eine randomisierte, Placebo (PLA)-kontrollierte klinische Doppelblindstudie in übergewichtigen-adipösen, nicht-diabetischen Probanden (21 Männer, 21 Frauen) durchgeführt. Die 12-wöchige EGCG/Res Supplementierung erhöhte die oxidative Kapazität in permeabilisierten Muskelfasern (ex-vivo) und bewahrte die in-vivo Fett-Oxidation (indirekte Kalorimetrie) im nüchternen und postprandialen Zustand verglichen mit der PLAGruppe, in der die Fett-Oxidation signifikant abnahm. Diese Resultate wurden durch eine erhöhte Expression von Genen und Proteinen (Muskelbiopsien vor und nach Intervention), die im oxidativen Energiestoffwechsel eine Rolle spielen, bekräftigt. Des Weiteren zeigten die VorherNachher Messungen, dass die Polyphenolsupplementierung das Ansteigen der TriglyzeridKonzentration im Plasma im Nüchternzustand und während des Testens einer fetthaltigen Mahlzeit verhinderte. Allerdings hat sich der Körperfettanteil, der Energieverbrauch und das Profil der Blut-Metaboliten im Nüchternzustand nicht verändert, obwohl sich die viszerale Fettmasse (DEXA) durch die Intervention tendenziell verringert hat. Diese beobachteten günstigen 
metabolischen Veränderungen spiegelten sich jedoch nicht in einer Verbesserung der peripheren, hepatischen und Fettgewebe-Insulinsensitivität wieder (2-Schritt hyperinsulinämischeuglykämische Clamp Technik), könnten aber langfristig eine Gewichtszunahme und Verschlechterung der Insulinresistenz vorbeugen (Kapitel 5).

Kapitel 6 beschreibt die Wirkung der 12-wöchigen Intervention mit EGCG und Res auf die Morphologie und das Transkriptom des weissen Fettgewebes. Vor und nach der Supplementierung wurden subkutane Fettgewebebiopsien vom Bauch genommen, um die Effekte auf die FettzellGrösse, den mitochondrialen Proteingehalt und das Genexpressionsprofil zu bewerten. Es zeigte sich, dass die EGCG/Res Supplementierung die durchschnittliche Adipozyten-Grösse, die Grössen-Verteilung und die Fettgewebe-Lipolyse (Mikrodialyse) im Vergleich zur PLA-Gruppe nicht beeinflusste. Jedoch suggerieren die Transkriptom-Resultate, dass die Intervention eine Suppression derjenigen Gen-Sets induziert, welche in Verbindung zur Adipogenese und Apoptose/Autophagie, Entzündung und der Immunantwort im Fettgewebe stehen. Dies könnte einer veringerten Fettzellerneuerung bzw. Entzündung des Fettgewebes in übergewichtigenadipösen Männern und Frauen entsprechen.

\section{Schlussfolgerungen}

Die in dieser Arbeit beschriebenen in-vitro und in-vivo Human-Studien, erweitern unser Wissen, ob und wie individuelle bioaktive Nahrungsinhaltsstoffe oder deren Kombinationen der Fettgewebe-Funktion, Insulinsensitivität und metabolischen Profilen zugute kommen. Jedoch werden die Behandlungseffekte von bioaktiven Stoffen/Kombinationen auf in-vitro AdipozytenEigenschaften wie z.B. Fetteinlagerung und Sekretionsprofil, durch die Differenzierungsbedingungen beeinflusst und lassen sich nicht ohne Weiteres auf die Fettgewebebiologie und den Energiestoffwechsel des Menschen übertragen. Nichtsdestoweniger wurde ein Anstieg der mitochondrialen Kapazität im Muskel und ein Erhalt der Ganzkörper-FettOxidation nach 12-Wochen EGCG/Res Supplementierung in übergewichtigen-adipösen Erwachsenen beobachtet. Trotz der metabolischen Verbesserungen, welche langfristig die zunehmende Insulinresistenz verzögern könnten, bedarf es weiterer Untersuchungen um zu zeigen, ob verschiedene Dosierungen oder Metaboliten von bioaktiven Substanzen zusammen mit Veränderungen des Lebensstils und/oder medikamentöser Behandlung effektiver wirken. Zusammengefasst lässt sich sagen, dass bioaktive Nahrungsinhaltsstoffe das Potenzial besitzen die Fettzellfunktionen und somit die Erfolge von Lebensstiländerungen zu verbessern, wenngleich deren Effekte vom Gesundheitszustand, Gewicht, der interstinalen Mikroflora, Umweltfaktoren und genetischer Veranlagung abhängen. Allerdings kann die Anwendung von bioaktiven 
Supplementen einen ungesunden Lebensstil, insbesondere einen erhöhten Konsum an energiereichen, Mikronährstoffe-armen Nahrungsmitteln und mangelnde sportliche Aktivität, nicht kompensieren (Kapitel 7). 
Valorization 


\section{What is valorization / knowledge valorization?}

The term valorization (surplus value, "Verwertung" in German) describes according to Marx's theory "the productive use of a resource, and more specifically the use or application of something (an object, process or activity) so that it makes money, or generates value, with the connotation that the thing validates itself and proves its worth when it results in earnings, a yield" [1]. Nowadays, the term valorization / knowledge valorization is also applied in academia and project management. According to the Dutch Ministry of Education, knowledge valorization is defined as "the transformation of knowledge into economic and social value". Its main purpose is the increase of the likelihood that scientific knowledge is more easily accessible and effectively used for public benefits [2]. Hence, here we discuss the social and economic relevance of the investigated aspects and the potential of the scientific findings with respect to their best use, and how specific population groups, health professionals and industries could benefit from applying the scientific knowledge described in this thesis.

\section{Social and economic relevance}

Multifactorial health conditions such as obesity and obesity-related cardiometabolic disorders are associated with complex interactions between the genome and various environmental factors. Diet composition, the intake of total calories, a reduced physical activity and a sedentary lifestyle are the major drivers of obesity. Hence, obesity has become an epidemic and is one of the major metabolic risk factors leading to non-communicable diseases (NCDs) like cardiovascular disease, type 2 diabetes mellitus (T2DM) and non-alcoholic fatty liver disease (NAFLD). According to the most recent study led by the Imperial College London and the WHO the number of obese adults increased 7-fold from 100 million in 1975 to 671 million in 2016 with another 1.3 billion adults being overweight. Shockingly, childhood and adolescent obesity increased 10-fold from 11 million in 1975 to 124 million in 2016 and additional 213 million overweight children and adolescent, which are likely to become obese [3, 4]. Evidence suggests that adipose tissue (AT) dysfunction rather than an increased total fat mass per se links obesity to its accompanying metabolic impairments such as hyperlipidemia and -glycaemia, chronic low-grade inflammation, endothelial impairment, and ectopic lipid accumulation that subsequently increase the prevalence of cardiovascular disease, T2DM and NAFLD. It is estimated that NCDs are the main cause of almost $86 \%$ of deaths in Europe, and $77 \%$ of the disease burden; and therefore, the WHO considers obesity to be the 5 th leading death risk worldwide [5]. Due to the growing prevalence of obesity and related NCDs, also in young individuals, the health care costs for obesity combatting strategies (like gastric banding, liposuction, gastroduodenal bypass, in addition to lifestyle changes) are increasing dramatically as well. The European Association for the Study of Obesity (EASO) points out that policymakers should consider 
that currently direct obesity-related costs range from 1.5 to $7 \%$ of health care expenditure in the EU [6] and diabetes accounts for approximately $10 \%$ of the total health care spending [7, 8]. Furthermore, these direct costs are accompanied by indirect costs (sick leaves, early retirement, use of social security funds, premature mortality, reduced productivity) summing up to $\sim € 390$ billion in 2010 for obesity and diabetes. In addition, to these direct and indirect costs, the continued consumption of highly processed, sugary, and fatty foods poses not only a threat to the global health care systems but also to the future economic growth. Morgan Stanley, a large scale commercial bank, estimates an $0.3 \% \mathrm{OECD}^{1}$ annual $\mathrm{GDP}^{2}$ growth in a high sugar scenario compared to a $2.2 \%$ with a low sugar scenario in 2035 ("The Bitter Aftertaste of Sugar" [9]). This awareness of the financial sector should further stimulate public sector education and private sector innovation, and foster academic investigations to tackle the "diabesity" (a term introduced for "coexistence of obesity and diabetes" due to the strong association between both conditions) effects on health costs and productivity [9], because currently government actions including food taxes and stricter regulations for food industry are not greatly effective in reversing the epidemic.

While surgical interventions provide immediate effect (the long-term effectiveness of surgeries remains still unclear [10]), they are associated with high costs, mortality, post-operative complications, and nutrient malabsorption, and therefore are not suitable as public health solution. Hence safe, effective, and easy-to-comply-with treatments and prevention modalities are needed to mitigate the development of obesity, paralleled by aiming to improve AT dysfunction and thereby metabolic impairments. Improving the unhealthy diets and physical activity levels will eliminate the main causes of obesity-related NCDs, and there are lifestyle interventions e.g., diabetes prevention studies (DPS, SLIM and EDIPS ${ }^{3}$ ) that have proven to be effective by obtaining a $\sim 50 \%$ reduction of the diabetes incidence rate. However, long-term compliance and weight maintenance is difficult to achieve and requires thus much more efforts by governments, health care services and food industries to implement these healthy lifestyles everywhere. One attempt to make these lifestyle interventions more effective is to supplement our diets with nutritional bio-actives. Nutritional bioactives, also called dietary bioactive components, can be defined as substances that are non-essential constituents in foods and dietary supplements, which improve cell, organ and overall body function and health. Unlike the essential nutrients, they (e.g. polyphenols, carotenoids, and polyunsaturated fatty acids (PUFA)) are not needed to maintain basic body functions, but they can have an impact on the health status [11]. Therefore, the identification and scientific substantiation of nutritional bio-

\footnotetext{
${ }^{1}<$ OECD $>$ Organization for Economic Cooperation and Development

$2<$ GDP $>$ Gross Domestic Product

$3<$ DPS $>$ Finnish Diabetes Prevention Study, $<$ SLIM $>$ Study on Lifestyle intervention and Impaired glucose tolerance Maastricht, $<$ EDIPS $>$ European Diabetes Prevention Study
} 
actives that enhance AT function and mitigate metabolic risk factors may improve the general health of the metabolically impaired population. The consumption of bio-actives represents an easily achievable preventive measure with moderate effects and the potential to reach most segments of the population, thereby having a high health impact. It seems also a promising business for the food and supplement industry (value of dietary supplements market in EU was $€ 7.2$ billion in 2015 [12]). However, although the scientific knowledge is growing and an increasing number of studies indicate beneficial effects of bio-actives on obesity-related metabolic diseases, inconsistent data are reported regarding the effects of individual nutritional bio-actives on AT biology, metabolic profiles, and energy metabolism. Moreover, well-controlled human intervention studies are scarce. It is therefore of interest to investigate how single and combinations of dietary bioactive components (with partly distinct mechanisms of action) impact adipocytes and AT function and whether such combinations could show favorable (additional / synergistic) effects on the metabolism of obese humans beside weight loss promotion.

The goal of this $\mathrm{PhD}$ project was to fill knowledge gaps in the field of nutritional bio-actives for the prevention and treatment of metabolic disorders by investigating the combinatory effects of the wellstudied polyphenols resveratrol (Res) and epigallocatechin-3-gallate (EGCG), the carotenoid lycopene and the PUFA eicosapentaenoic acid (EPA) in a unique human cellular model and in a placebo-controlled human intervention study with overweight-obese subjects. Therefore, the translational research described in this thesis was structured going from method development and validation to in-vitro experiments and finally in-vivo intervention studies.

\section{Relevance of studies}

The studies conducted in this thesis expand the current knowledge regarding the potential role of nutritional bio-actives on AT substrate and energy metabolism, thereby contributing to the fundamental and applied research ambitions according to the strategic program 2017-2021 of Maastricht University (UM, section Research, Action: "Maintain a healthy balance between fundamental and theme-driven research"). The thesis itself is part of the intended high output of $\mathrm{PhD}$ theses, which fosters education, attracts researchers / students, and keeps the university competitive on the national and international level [13]. On the one hand, in-vitro models are useful and can be effectively applied for high-throughput screening of the metabolic health effects of nutritional bioactives. However, considering nutrient-nutrient interactions, as well as nutrient-gut-microbiome interactions and systemic bioavailability of bio-actives, human intervention studies are not only necessary but also remain the gold standard in studying the effects of nutritional bio-actives on human health and well-being. 
On the other hand, nutritional human studies are challenging and require further standardization, refinement, and careful execution to high standards like the conduct in accordance with GCP, rigorous monitoring, and well-controlled and documented dietary intake. In more detail, cellular models allow for the investigation of several components, their derivatives / metabolites [14], and combinations in a fast, easy, and well-controlled manner and thus are valuable tools for highthroughput screening and mechanistic understanding. Because evidence is accumulating that primary human multipotent adipose-derived (hMADS) cells retain their donor characteristics and can be differentiated in-vitro into adipocytes, they represent a convenient AT model to investigate in-vitro responses in metabolically distinct groups. The presented research has corroborated that this process is responsive to individual nutritional bio-actives and their combinations. The hypothesis for the conducted studies was that combinations were more effective than individual compounds regarding their influence on lipid accumulation, various AT functions and metabolic parameters such as insulin sensitivity. This is suggested by a reduced lipid accumulation and pro-inflammatory secretion in in-vitro differentiated adipocytes (Lyc/Res), a prevented decrease of fat oxidation invivo and increased skeletal muscle mitochondrial capacity (EGCG/Res) and modified transcriptional patterns in adipocytes, AT, and muscle after treatment with combinations. However, these findings were not reflected by increased energy expenditure, changed food intake, improved insulin sensitivity or white AT loss after 12 weeks supplementation. Therefore, such results should not be advertised as long-term weight loss solutions to consumers, but they may indicate that the supplementation of "multi-bio-actives" combined with a healthy lifestyle could help combat metabolic dysfunction accompanied by excessive weight. In addition, to the putative health benefits of correcting metabolic parameters this is an attractive business opportunity comparable to established multi-vitamin/mineral products. Furthermore, attention must be paid to high and overdosing of nutritional bio-actives and their possible interactions with other nutrients, semiluxury foods and drugs. The impact of high doses and such interactions may be detrimental for human health and well-being and needs to be further investigated and communicated to healthcare professionals advising on lifestyle as well as directly to consumers. Evidence is accumulating that in-vivo effects of dietary bioactive components seem dependent on the metabolic status, gut microbiota composition, and gender of the subjects and may be organspecific. Thus, a generalization that supplementation with such bio-actives is beneficial for human health first requires a better characterization of subject subgroups such as responders / nonresponders and reliable biomarkers. 


\section{Target Groups, Activities, and Products}

In-vitro and human intervention studies provide detailed knowledge about toxicity, effectiveness, and safety of different dosages of bio-actives during short and longer-term treatment / supplementation. This contributes to the establishment of recommended intake values [11] and scientifically sound health effects of these constituents. Defining recommended intakes of non-essential nutritional bio-actives is yet difficult due to contradictory results of human intervention studies. Several reasons may account for this e.g., differences in i) the extent of obesity and body composition, ii) habitual dietary intake, iii) physical activity levels, iv) the compliance of the subjects, v) the genetic background, and vi) gut microbiota diversity. Consequently, for future investigations larger, and longer studies are necessary, carefully adjusted for confounding or interacting factors e.g., enrolling well-characterized subjects with different metabolic risk factors, and including adequate controls, to identify beneficial effects for subpopulations and to establish recommended intake levels. Such studies will provide valuable information for industries that commercialize dietary supplements and functional foods and will help raise the awareness of a healthy lifestyle including the consumption of nutritional bio-actives containing fruits and vegetables [11]. A critical evaluation of beneficial and non-effective nutritional research outcomes by (inter-)national organizations and independent institutions e.g., EFSA ${ }^{4}$ should be mandatory to position results in the right context and protect consumers from health claims without sufficient scientific evidence. In addition, such overarching evaluations include inconsistencies in data from human nutrition studies e.g., as reported for Res [15] and addressed in a recent study by Kjaer et al. [16] and shows that drawing conclusions from a limited number of studies in different sub-groups are misleading. Therefore, defining new biomarkers for the intake of bio-actives and their impact on metabolic health with the help of -omics technologies [17] can give indications regarding sub-populations which benefit from the supplementation with nutritional bio-actives and subsequently will promote more directed prevention / intervention strategies. Another option to identify possible target groups is to make all generated data sets accessible to institutes and companies that integrate the knowledge into the evolving "big data" approach. This is in accordance with another objective of knowledge valorization: to rapidly share results with the scientific community and health professionals through publication in international peer-reviewed journals and presentation at international scientific conferences. Furthermore, sharing of research outcomes in layman's terms, in accessible university media channels (www.uctv.tv; https://www.mumc.nl/en/research) can be a suitable way to translate and spread scientific knowledge for the society.

\footnotetext{
${ }^{4}<$ EFSA $>$ European Food and Safety Authority
} 
The cell model chapters emphasize that further improvement, standardization, and validation of invitro models are necessary to i) better mimic in-vivo micro-environments (3D structures, co-cultures, nutrient supply (supra-physiological vs. physiological nutrient concentrations)), ii) generate reproducible results (e.g. pro- or anti-adipogenic effects of Res) in independent labs and experiments, and iii) enable the transition from in-vitro to in-vivo results. These cost-effective in-vitro settings could eventually reduce or skip future animal experiments (as done in this thesis). The in-vitro assay established herein to investigate lipid droplet accumulation is also a good starting point to develop a high-throughput commercial screening method. This method could facilitate a faster identification of dietary components regarding their effects on adipocyte lipid droplets biology from individual donors. Moreover, it could determine additive or synergistic effects of combinations and unravel underlying mechanisms, before investigating selected combinations in more sophisticated and expensive human in-vitro models, or clinical trials.

The in-vitro anti-inflammatory and anti-adipogenic effects of the bio-actives described in this thesis could however not be translated in-vivo into beneficial longer-term health effects (including improved insulin sensitivity and AT morphology). This could be due to the huge complexity of the potentially involved mechanisms (interactions of numerous cell types and organs) or due to the enrollment of healthy overweight-obese subjects, which could partly explain some discrepancies described in the literature. Nevertheless, the multi-organ impact and cross-talk of nutritional bioactives in-vivo will hamper that implications from even improved in-vitro cell-based studies (human cell lines, organoids, 3D-/co-culture models) add significantly to our understanding of how nutritional bio-actives contribute to human metabolic health.

However, in-vitro data are valuable for the generation of intellectual property (IP). Hence the inhibitory effects of the combinations on the lipid accumulation in differentiating adipocytes were already filed in a patent application before publication of our results. In contrast, human intervention studies offer only small possibilities to create IP because they need a primary objective and an ethical justification to conduct the investigation, which is mostly based on previous research findings. Unfortunately, the supplementation with the polyphenol combination EGCG/Res did not show additive or synergistic (more likely to be patentable) effects when comparing results with other human invention studies investigating individual polyphenols.

The studies, experiments, and analyses described in this thesis were conducted at the department of Human Nutrition and Health at DSM Nutritional Products (Basel, Switzerland), the research department of Human Biology (NUTRIM, Maastricht University, Netherlands), and the Nutrition, Metabolism, and Genomics Group (Division of Human Nutrition, Wageningen University, Netherlands). Moreover, the human study was supported by a grant of the Alpro Foundation (Gent, 
Belgium). This sets a good example for a fruitful collaboration and networking between universities and nutrition industry partners, which promotes applied research and joint utilization of resources for a faster valorization of results. Taken together, the results of this thesis were only achieved due to these collaborations and importantly, they give novel impulses for further investigations in the field of nutrition.

\section{References}

1. Wikipedia. Valorisation. Marxian economics [ free encyclopedia] 200517 September 2017 [cited 2017 September 10th].

2. Hladchenko, M., Knowledge valorisation: A route of knowledge that ends in surplus value (an example of the Netherlands). International Journal of Educational Management, 2016. 30(5): p. 668-678.

3. Abarca-Gómez, L., et al., Worldwide trends in body-mass index, underweight, overweight, and obesity from 1975 to 2016: a pooled analysis of 2416 population-based measurement studies in 128.9 million children, adolescents, and adults. The Lancet, 2017.

4. WHO. Tenfold increase in childhood and adolescent obesity in four decades: new study by Imperial College London and WHO. [Press Release] 2017 [cited 2017 October 12th].

5. Erixon, F., L. Brandt, and M. Krol. Investing in Obesity Treatment to Deliver Significant Healthcare Savings: Estimating the Healthcare Costs of Obesity and the Benefits of Treatment. 2014 [cited 2017 October 16th].

6. EASO. European Association for the Study of Obesity: Tackeling Obesity Together. 2017 [cited 2017 October 16th].

7. Hex, N., et al., Estimating the current and future costs of Type 1 and Type 2 diabetes in the UK, including direct health costs and indirect societal and productivity costs. Diabet Med, 2012. 29(7): p. 855-62.

8. HEAL, Health and Environment Alliance: Health costs in the EU - How much is related to EDCs? 2014, HEAL: http://www.env-health.org/news/latest-news/article/health-costs-in-the-euhow-much-is.

9. Bartsch, E., Sustainable Economics - The Bitter Aftertaste of Sugar. 2015, MORGAN STANLEY RESEARCH.

10. Colquitt, J.L., et al., Surgery for weight loss in adults. Cochrane Database Syst Rev, 2014(8): p. Cd003641.

11. Wallace, T.C., et al., Dietary Bioactives: Establishing a Scientific Framework for Recommended Intakes. Advances in Nutrition: An International Review Journal, 2015. 6(1): p. 1-4.

12. Statista. Value of the dietary supplements market in Europe in 2015 and 2020. 2017 [cited 2017 October 29th].

13. Maastricht-University. Maastricht University - Community at the CORE: Strategic programme 2017-2021. 2016 [cited 2017 October 09th].

14. Gomez-Zorita, S., et al., Pterostilbene Inhibits Lipogenic Activity similar to Resveratrol or Caffeine but Differently Modulates Lipolysis in Adipocytes. Phytother Res, 2017.

15. Liu, K., et al., Effect of resveratrol on glucose control and insulin sensitivity: a meta-analysis of 11 randomized controlled trials. Am J Clin Nutr, 2014. 99(6): p. 1510-9.

16. Kjaer, T.N., et al., No Beneficial Effects of Resveratrol on the Metabolic Syndrome: A Randomized Placebo-Controlled Clinical Trial. J Clin Endocrinol Metab, 2017. 102(5): p. $1642-$ 1651.

17. Korsholm, A.S., et al., Comprehensive Metabolomic Analysis in Blood, Urine, Fat, and Muscle in Men with Metabolic Syndrome: A Randomized, Placebo-Controlled Clinical Trial on the Effects of Resveratrol after Four Months' Treatment. Int J Mol Sci, 2017. 18(3). 
Acknowledgements

Danksagungen 
Not easy to write them but somehow it is a huge relief to compile the acknowledgements. My PhD thesis was accepted and approved to be defended. So, saying "thank you" to all the people who supported me continuously during this long-lasting, educational, and "juicy-nutty" period is worth some lines. It would not have been possible without them. The order of appearance and number of words do not correlate at any level with my appreciation and the importance of the people and in case I have not mentioned someone specifically please feel addressed by the "all";).

A big thank you to my supervisors and co-supervisors who helped me to stay on track, enabled me to complete this "part-time" undertaking, for their time spent reading my always too long drafts and especially for all their crucial comments and helpful feedback.

A special thanks to Joseph for his guidance and challenging questions over the years and for giving me the freedom to make my own mistakes when setting up and carrying out the experiments. I much appreciated the fruitful, not always science-related, discussions over a coffee in Kau or in the academic atmosphere of the "Bio-Zentrum" after you retired. Dear Wim, I was always wondering why you supported this strange German woman living and working far away in Switzerland, but I am more than grateful that you never stopped to push me forward and introduced me to the Human Biology Group at University Maastricht. Thus, also many thanks to Ellen and Johan for supervising, supporting my stay at your department and the scientific insights during our discussions. Johan, I greatly appreciated our inspiring conversations, your always very creative approach, and the editorial additions.

Of course, I would also like to thank the members of the assessment committee, Prof. Dr. Edwin C.M. Mariman, Prof. Dr. Aalt Bast, Prof. Dr. Michael Müller, Prof. Casper G. Schalkwijk and Prof. Hubert Vidal for reading and assessing my manuscript and providing helpful comments to improve the print version.

Overall, I would like to thank my family and all my friends for listening to my complaints about the lack of sleep and never having enough time, the overwhelming tasks ahead and for enduring my sleep deprived and stressed moods and cheering me up whenever needed.

Zusammengefasst in Deutsch: ein riesen Dank an meine Familie und all meine Freunde, dafür dass ihr mich während der ganzen Zeit immer wieder betüddelt habt und meine Launen und Beschwerden über Schlaf-und Zeitmangel und zu viel zu tun, ertrugt.

In addition, many thanks to the DSM crowd: Werner, the internal DSM supporter in chief, who always found a good solution for the trickiest organizational issues; Regina and Igor for the 
financial and moral support, all the HNH R\&D community for sharing their knowledge and the successor of Werner for allowing me to finalize my thesis under the new regime.

Surprisingly I have experienced quite a few changes in management during the $\mathrm{PhD}$ period. This is either an indication for a high turnover at DSM or a sign that I needed long to get all the required publications together ;-). Nevertheless, I was super lucky, that my direct line managers and in a broader sense DNP-HNH were so favorably attuned to this side project. So, Iris and Jonas, I want you to know how much indeed I value(d) your support.

Without the technical and hands-on help, I probably would still be busy with RTFM, pipetting and reading plates. Therefore, many thanks to Peggy and Janina for teaching me the basic lab skills far way back, and to Eliane, Nicole S., Karin, and Nathalie for their help with the ArrayScan issues, carotenoid handling, cell culture and methodology support and all the other tips and tricks. Christine, my dear little "queen of $\mathrm{f}$....ing everything" what would I have done without your tedious and speedy hands which processed all the RNA samples with such diligent care. Thanks for all the hours you invested and being such a good bicycle companion. Tina, a pity that we do not share a lab anymore. I miss our working sessions with the "background" music, but I am really happy you are still around as (in)sanity indicator. Ladies, I have had / have so much fun working with you! Furthermore, I would like to thank Nicole H. and Yvonne E. of the Human Biology Group at the $\mathrm{UM}$, for sharing their office and lab space with me and for their help with the cell and tissue work. Also thanks to the other members of the group / people on the floor, especially to Desiree, for their hospitality, organisational help, and the funny Friday evening drinks. Dear Jasper, I am very thankful that I got the chance to be involved in your ALPRO studies, that you endured and answered all my questions, and have driven forward the publishing of the human study data. Thanks also to Kenneth for the isolation of the hMADS cells and to the study subjects for their time and biopsies.

Timolein, I am eternally grateful for everything you've helped me with and your adorable good spirit often balancing my irritated and "confuzzled” state. And Özgür, I cannot thank you enough for initiating the whole undertaking by introducing me to Wim, for the medical input and the proof reading of the manuscript. My best buddy Paulito, listing everything I appreciate since we know each other is beyond the scope of these lines, so in short: thanks for your encouragement and friendship ;).

Moreover, many thanks to all my friends: Katha, Kit, Käthy, Tina, Eliane, Iris, Kathrin, Franz, Laura, Michi, Daniel, Sofia, Gisella and Co, for keeping me sane and fit by persuading me to take 
holidays, go climbing, skiing, running, cycling, hiking and more, and take over godchild sitting, despite the work load and for advising on Dutch $\mathrm{PhD}$ defense traditions, helping with the cover design and for consuming a lot of coffee (procafination!), tea and Nuss-Mus.

Despite all issues, I am grateful that WT was part of my life in the last 3 years of the PhD period, that he introduced me to road racing and broadened my horizon regarding interpersonal communication.

Nicht zu vergessen, ein grosser Dank für den Halt den mir meine «Drei Grazien» in allen Lebenslagen geben und den Spaß, den wir zusammen haben. Ein Merci auch an deren bessere Hälften, die all unseren «Unfug» ertragen.

Zum Schluss, Mutti und Papa, danke ich Euch für all die Unterstützung und Liebe, die Ihr mir gegeben habt, während des für Euch verrückt klingenden Studienganges, bei den Umzügen und all den anderen Flausen und Aktivitäten auf meinem bisherigen Weg. Ich schätze mich glücklich solche eine tolle Familie «Flodder» zu haben.

Last but not least, there are many more things I am grateful for and people I could thank, but it may be also a good point in time to acknowledge the existence of the universe and all the evolutionary events that led to our emergence, and to science-driven societies. I hope that these will be able to save the planet and us by holding back "idiocracy"... May the force be with us ;-D 
Publications

231 


\section{Published Manuscripts}

Warnke I, Goralczyk R, Fuhrer E, Schwager J: Dietary constituents reduce lipid accumulation in murine C3H10 T1/2 adipocytes: A novel fluorescent method to quantify fat droplets. Nutrition \& metabolism 2011, 8(1):30.

Warnke I, Jocken JW, Schoop R, Toepfer C, Goralczyk R, Schwager J: Combinations of bioactive dietary constituents affect human white adipocyte function in-vitro. Nutrition \& metabolism 2016, 13:84.

Most J, Timmers S, Warnke I, Jocken JW, van Boekschoten M, de Groot P, Bendik I, Schrauwen P, Goossens GH, Blaak EE: Combined epigallocatechin-3-gallate and resveratrol supplementation for $12 \mathrm{wk}$ increases mitochondrial capacity and fat oxidation, but not insulin sensitivity, in obese humans: a randomized controlled trial. Am J Clin Nutr 2016, 104(1):215-227.

\section{Manuscripts in preparation}

Most, J*, Warnke, I*, Boekschoten, M, Jocken, JWE1, de Groot, P, Friedel, A, Bendik, I, Goossens, GH, Blaak, EE: The effects of polyphenol supplementation on adipose tissue morphology and gene expression in overweight and obese humans. Submitted to Adipocyte.

Warnke, I, Schwager, J, Verboven, K, Schoop, R, Toepfer, C, Blaak, EE, Jocken, JWE: Effects of combining resveratrol with lycopene on functional features of in-vitro differentiated human white adipocytes. To be submitted.

Warnke, I, Schwager, J, Saris, W. Blaak, EE, Jocken, JWE: Modification of white adipose tissue biology by nutritional bio-actives. To be submitted.

*These authors contributed equally to this work 
Curriculum Vitae 
Ines Warnke was born on May $2^{\text {nd }}$ in 1979 in Ludwigslust. She attended secondary school at the "Goethe-Gymnasium" in Ludwigslust, where she chose the advanced courses chemistry and mathematics. After her graduation in 1997, she undertook a voluntary social year at the hospital "Stift Bethlehem" in her home town. From 1998 to 2005 Ines completed her undergraduate and graduate studies in "Nutritional Science" at the University of Potsdam, Germany, where she also worked as Student worker at the Max-Planck-Institute of Molecular Plant Physiology. She conducted the experimental work for her Diploma thesis entitled "Folate requirement and synthesis by commensal bacteria of the human large intestine" at the Rowett Research Institute in Aberdeen, Scotland, from 2003 to 2004 and graduated with honors in 2005.

Since September 2005, Ines has been working in the Department of Human Nutrition and Health at DSM Nutritional Products in Basel, Switzerland. After 5 years of working in the field of biological assay development as Associated Scientist, she was also trained as Clinical Research Associate to monitor human clinical studies. In 2011, she initiated the set-up of her PhD thesis based on results obtained in a project related to lipogenesis inhibition. Consequently, she became a part-time PhD student at the Department of Human Biology (NUTRIM, School of Nutrition and Translational Research in Metabolism), at Maastricht University in July 2013. The research performed during this collaborative project work, supervised by Prof. Dr. Wim Saris, Prof. Dr. Ellen Blaak and Dr. Joseph Schwager, focused on dietary constituents influencing lipid accumulation in fat cells and is described in this thesis entitled: "Modulation of white adipose tissue biology and metabolic profiles in humans by nutritional bio-actives". In May 2015, Ines was promoted and became Scientist at DSM.

In her free time, Ines is keen on spending some energy during sportive activities like cycling, skiing, climbing, and teaching Pilates. Moreover, she enjoys sociable meetings with friends and her family, shopping on the local food market and tinkering. 
